Supporting Information

For

\title{
Threshold of Thioglycoside Reactivity Difference Is Critical for Efficient Synthesis of Type I Oligosaccharides by Chemoselective Glycosylation
}

\author{
Nitish Verma, ${ }^{\mathrm{a}, \mathrm{b}, \mathrm{c}}$ Zhijay Tu, ${ }^{\mathrm{a}}$ Ming-Shiuan Lu, ${ }^{\mathrm{d}}$ Shih-Hao Liu, ${ }^{\mathrm{a}}$ Septila Renata ${ }^{\mathrm{acc}, \mathrm{e}}$ Riping \\ Phang, ${ }^{\mathrm{d}}$ Peng-Kai Liu, ${ }^{\mathrm{f}}$ Bhaswati Ghosh, ${ }^{\mathrm{a}}$ and Chun-Hung Lin ${ }^{\mathrm{a}, \mathrm{c}, \mathrm{d}, \mathrm{f}, *}$
}

anstitute of Biological Chemistry, Academia Sinica, No. 128, Academia Road, Section 2, Nankang, Taipei 11529, Taiwan

${ }^{b}$ Department of Chemistry, National Tsing Hua University, No. 101, Section 2, Kuang-Fu Road, Hsinchu 300044, Taiwan

${ }^{c}$ Chemical Biology and Molecular Biophysics, Taiwan International Graduate Program, Institute of Biological Chemistry, Academia Sinica, No. 128, Academia Road, Section 2, Nan-kang, Taipei 11529, Taiwan

${ }^{\mathrm{d}}$ Department of Chemistry, National Taiwan University, No. 1, Section 4, Roosevelt Road, Taipei 10617, Taiwan

${ }^{\mathrm{e}}$ Institute of Bioinformatics and Structural Biology, College of Life Science, National Tsing Hua University, No. 101, Section 2, Kuang-Fu Road, Hsinchu 300044, Taiwan

Institute of Biochemical Sciences, College of Life Science, National Taiwan University, No. 1, Section 4, Roosevelt Road, Taipei 10617, Taiwan

*Corresponding author E-mail: chunhung@gate.sinica.edu.tw 
Table of Contents

I. Supporting Schemes, Figures, and Tables

S1

Scheme S1. Synthesis of Type I LacNAc Hexasaccharide 36 by [4 + 2] Glycosylation

Between Type I LacNAc Tetrasaccharide Donor 25 and Anomeric OTBS-Protected

Disaccharide Acceptor 35.

S1

Scheme S2. Synthesis of Donor 45. ........................................................................... 1

Scheme S3. Synthesis of GlcNTCA Building Block 48. ............................................... S1

Scheme S4. Synthesis of Disaccharide Building Blocks 2 and 35. ............................... S2

Scheme S5. Synthesis of Several Type I LacNAc Disaccharide Donors (4, 6, 7, and 8) and

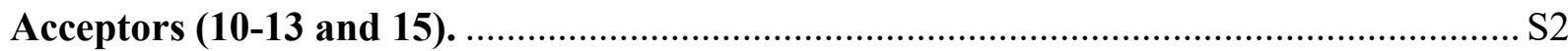

Scheme S6. Synthesis of Several Type I LacNAc Disaccharide Donors (1, 3, and 5) and

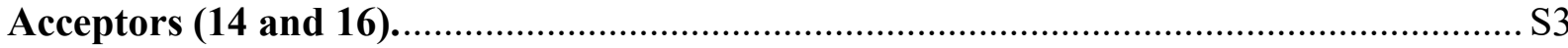

Scheme S7. Synthesis of Type I LacNAc Disaccharide Donor 9. ..................................... S3

Scheme S8. $[2+2]$ Chemoselective Glycosylation by Using a Preactivation Method....... S3

Scheme S9. Synthesis of Type I LacNAc Tetrasaccharide Acceptor 37 ......................... S4

Figure S1. Reference Donors Used for the RRV Determination. .................................. S4

Figure S2. Linear Correlation was Observed Between Estimated $\ln \left(R_{R} V_{D}\right)$ and Yield of

Tetrasaccharide Products Shown in Table 1.............................................................. 5

Table S1. Reactivity Ratio and RRV of Disaccharides 1-9........................................... S6

Table S2. Reactivity Ratio and RRV of Disaccharides 10-16....................................... S6

Table S3. Listed Estimated $\ln \left(R_{R V}\right)$ of the $[2+2]$ Glycosylations that are Shown in

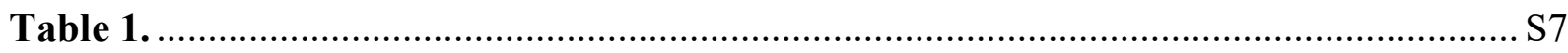

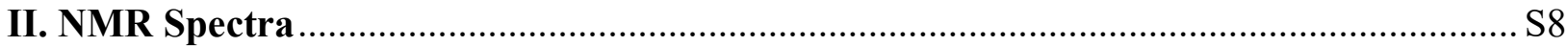

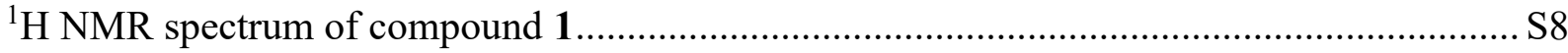

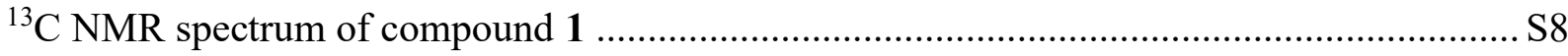

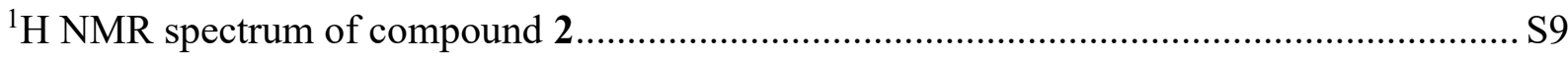

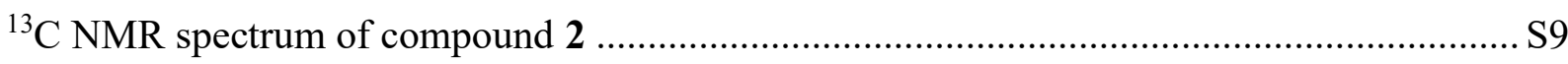

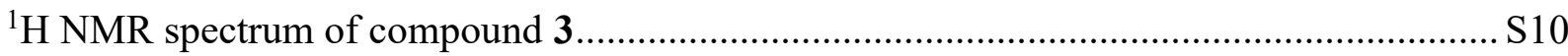

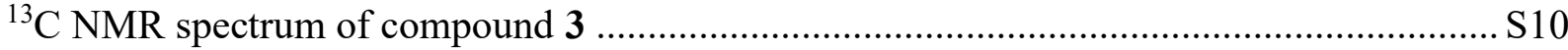

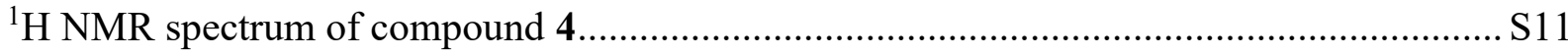

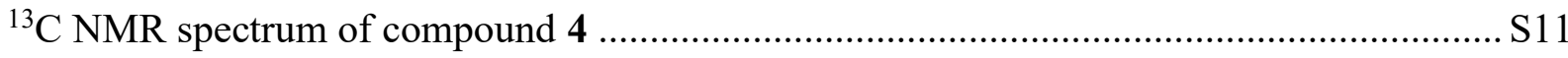

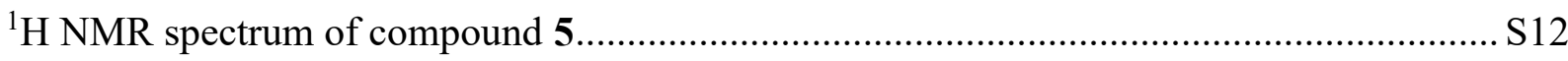

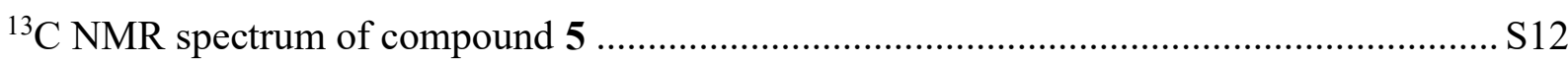

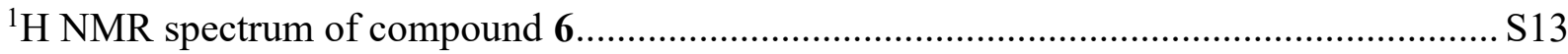




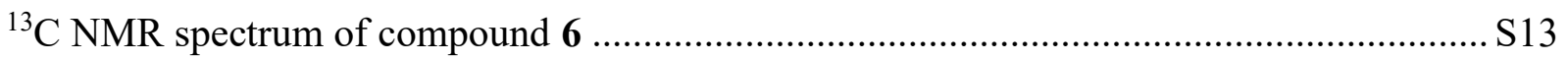

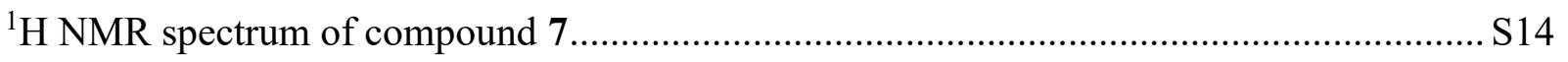

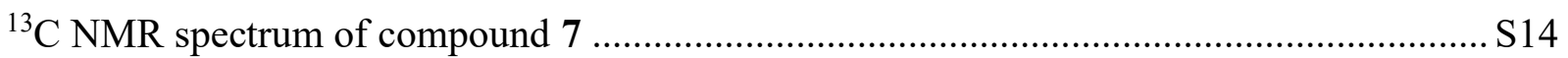

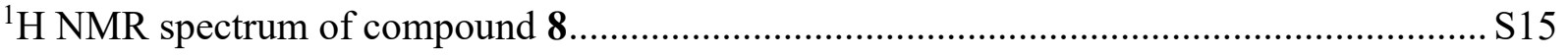

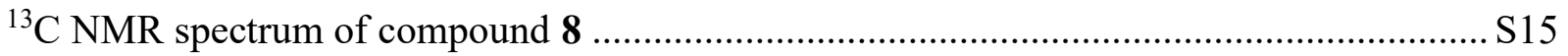

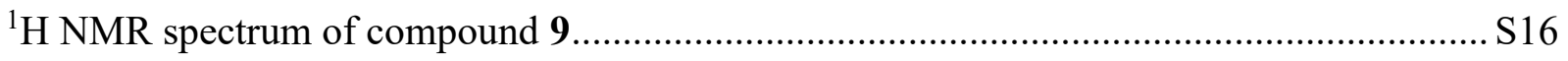

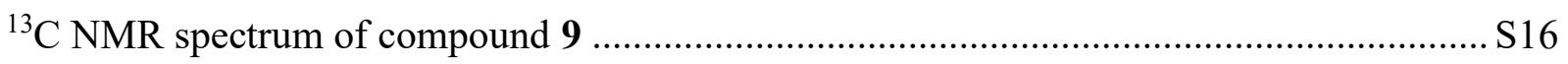

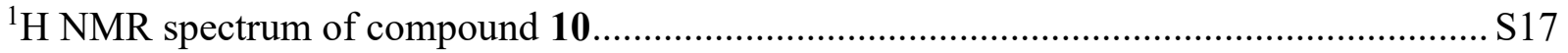

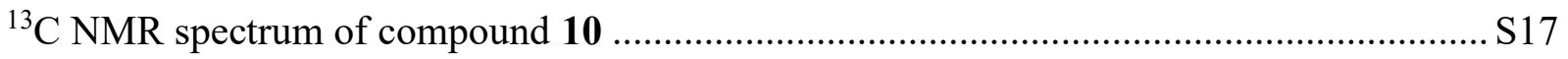

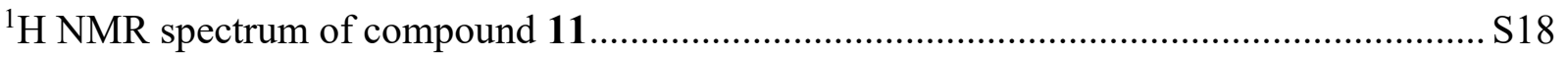

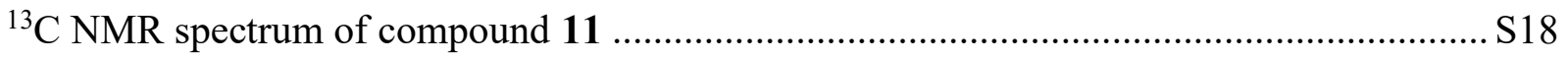

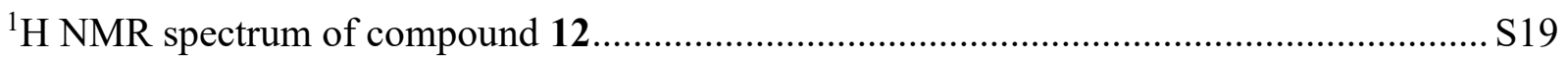

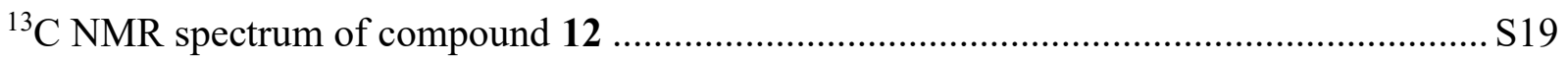

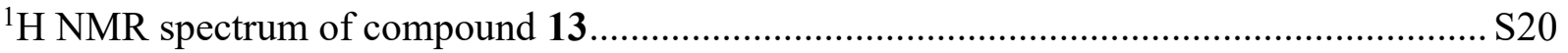

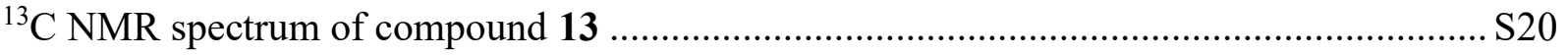

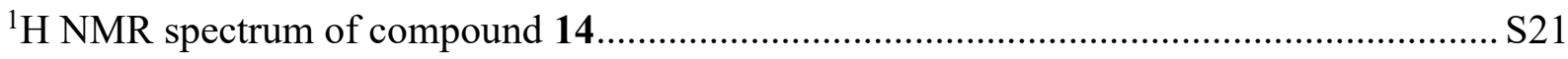

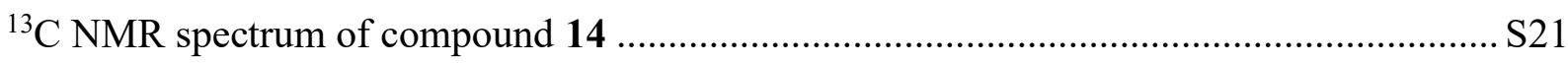

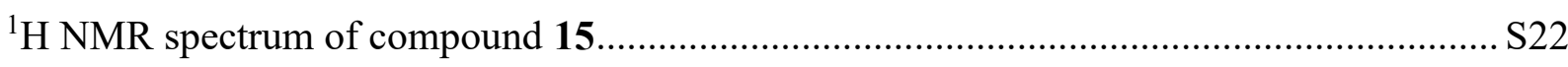

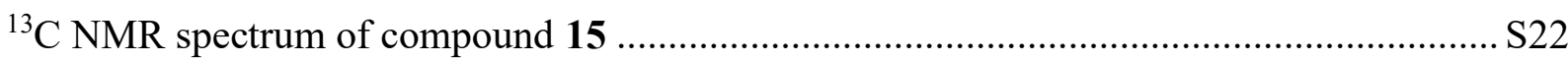

${ }^{1} \mathrm{H}$ NMR spectrum of compound 16........................................................................... S23

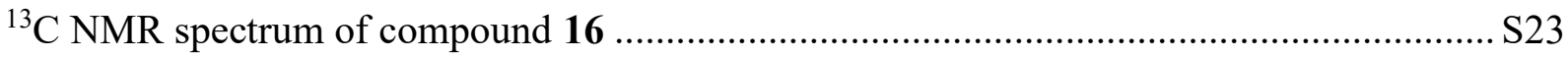

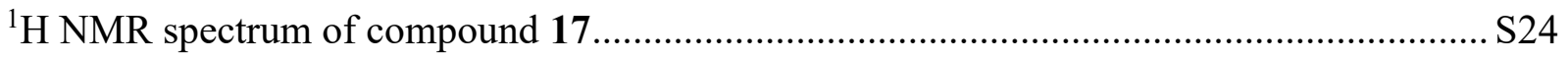

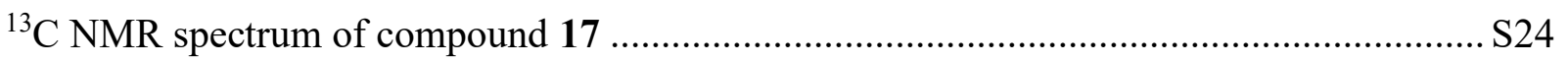

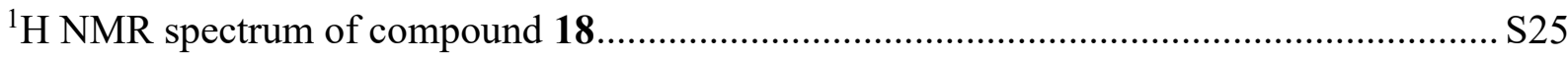

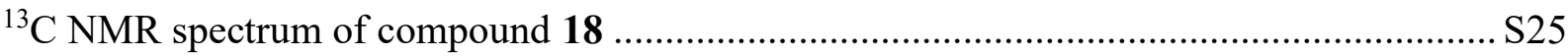

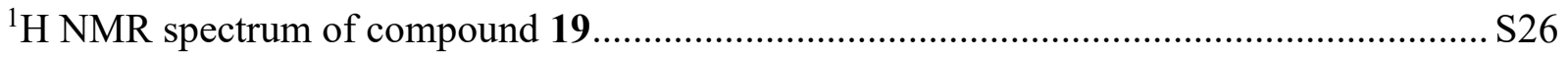

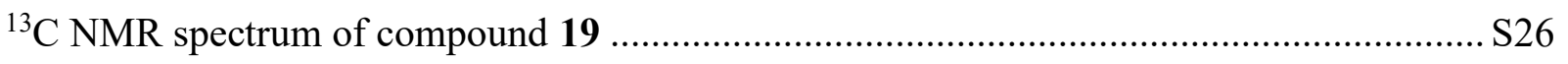

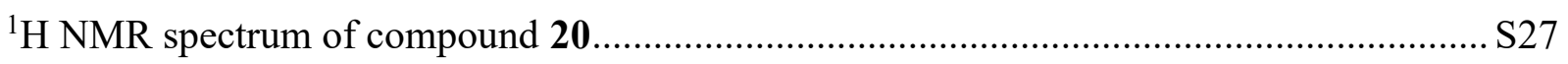

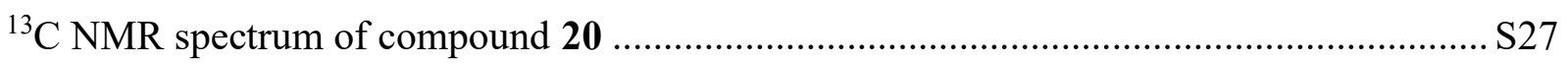

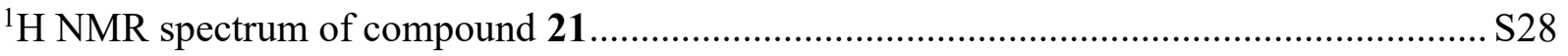

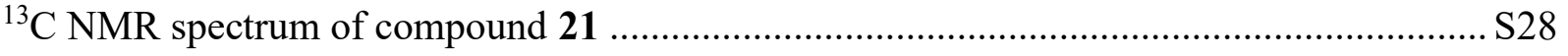

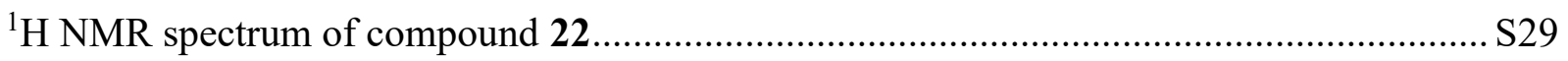




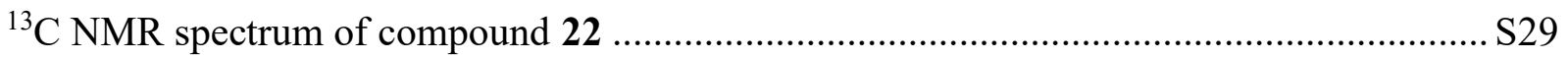

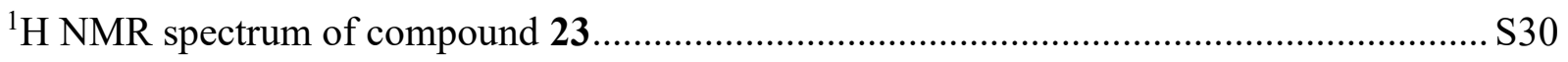

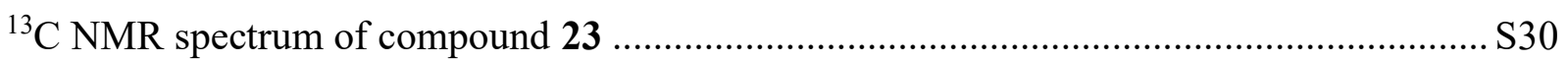

${ }^{1} \mathrm{H}$ NMR spectrum of compound 24 ................................................................................ S31

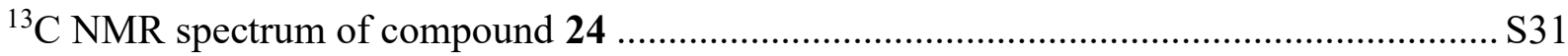

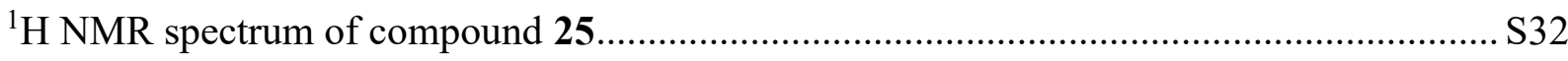

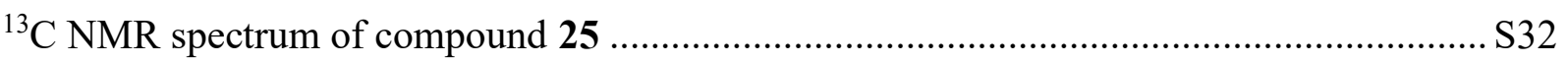

${ }^{1} \mathrm{H}$ NMR spectrum of compound 26................................................................................. S33

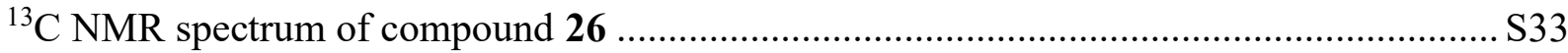

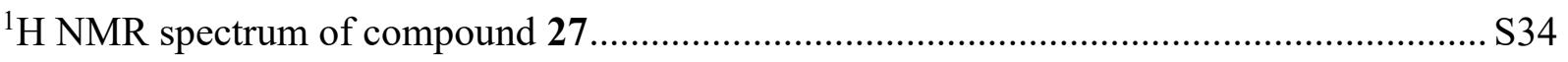

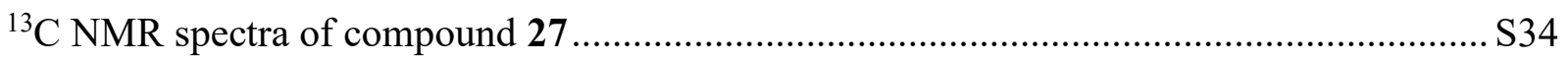

1D-Selective TOCSY NMR spectra of compound 27 ………........................................... S35

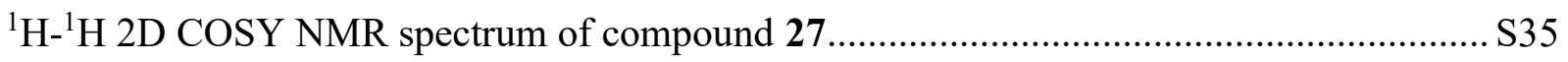

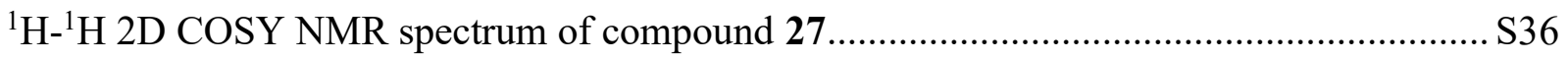

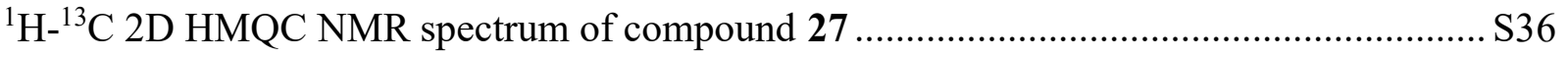

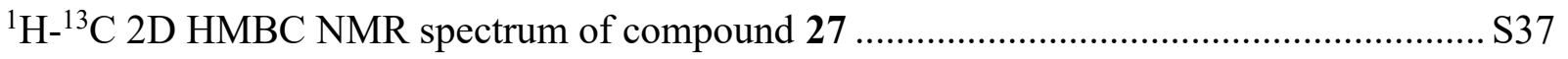

${ }^{1} \mathrm{H}$ NMR spectrum of compound 28

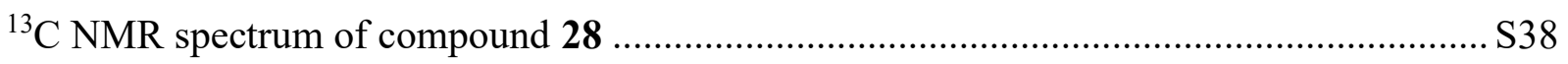

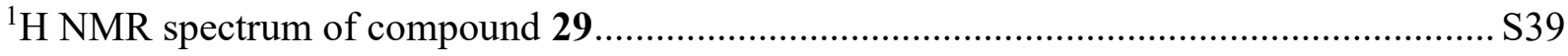

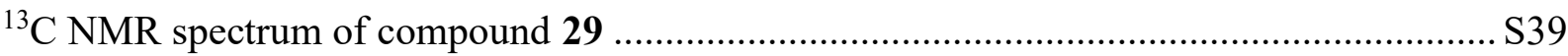

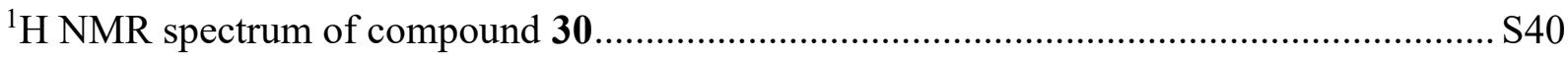

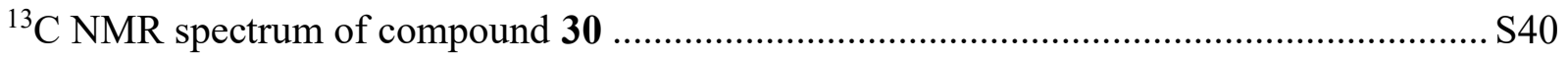

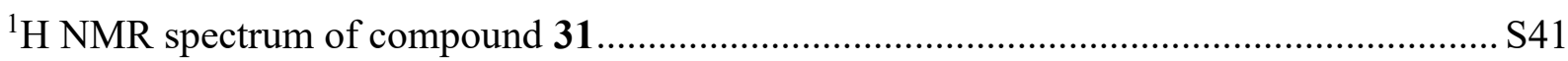

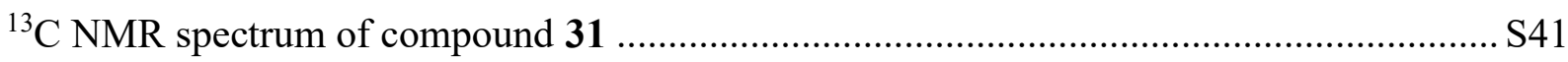

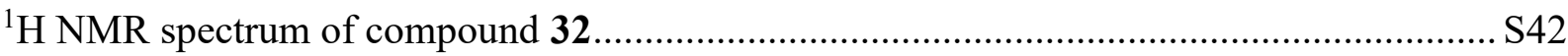

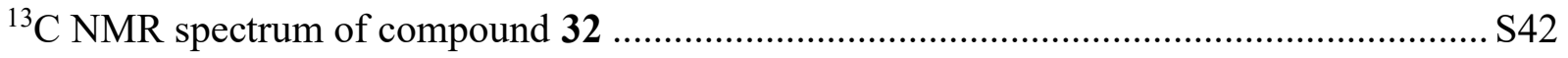

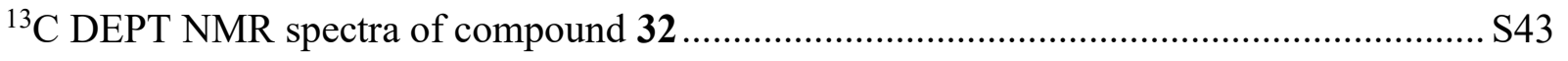

1D-Selective TOCSY NMR spectrum of compound 32 .................................................. S43

1D-Selective TOCSY NMR spectrum of compound 32 ……........................................... S44

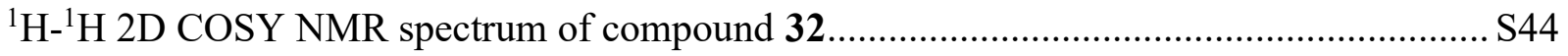

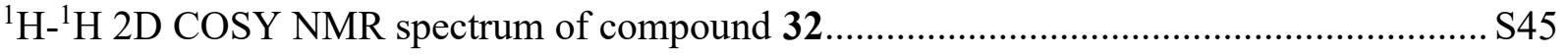

${ }^{1} \mathrm{H}-{ }^{1} \mathrm{H}$ 2D COSY NMR spectrum of compound 32 


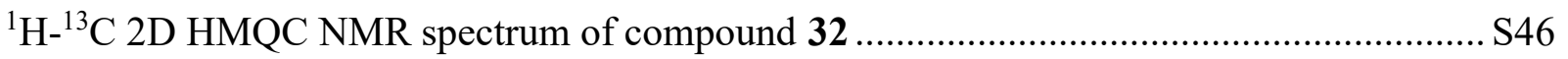

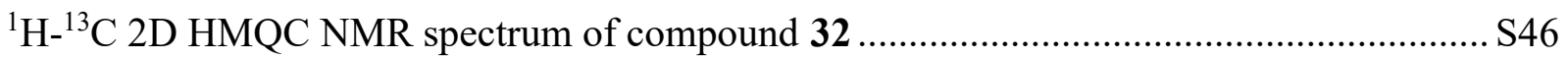

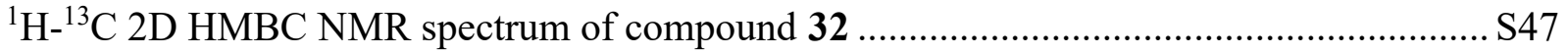

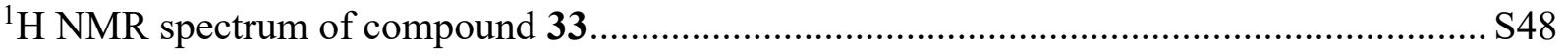

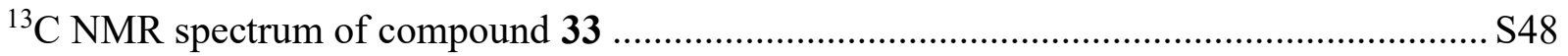

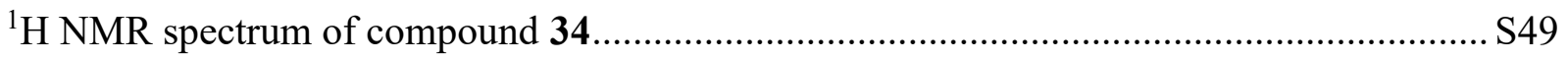

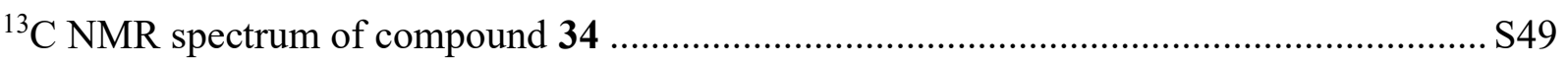

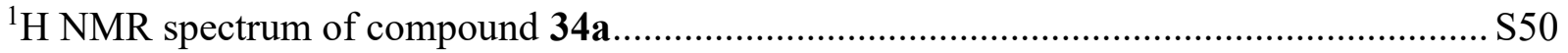

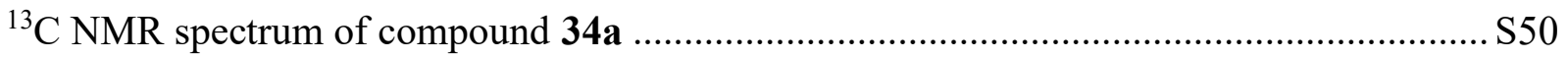

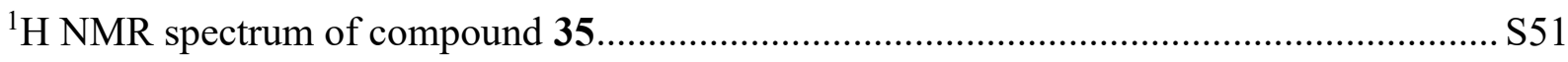

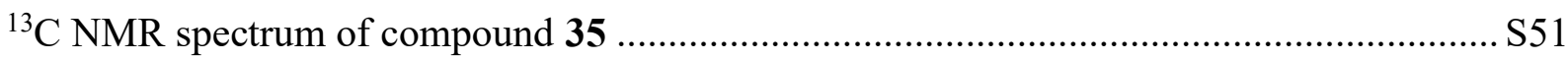

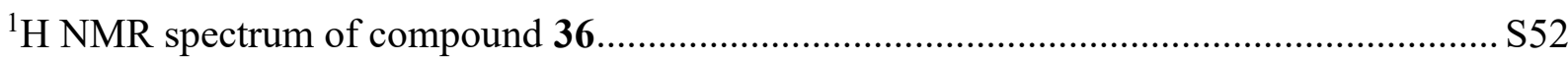

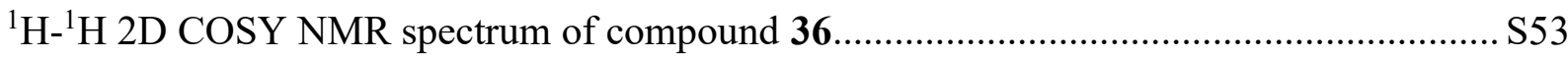

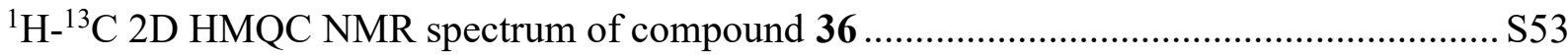

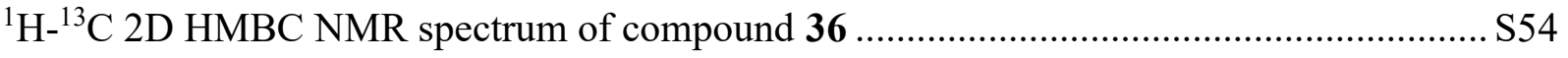

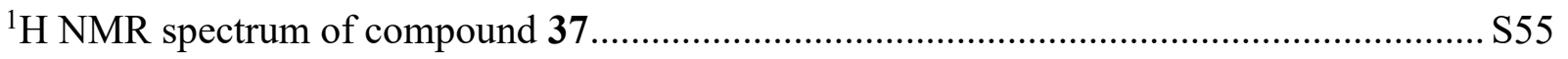

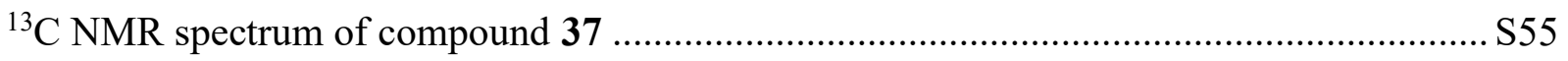

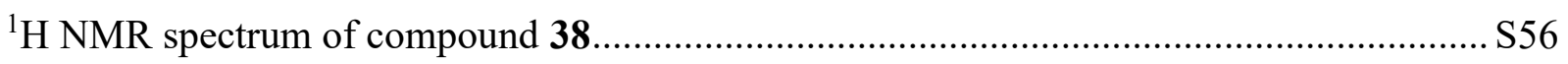

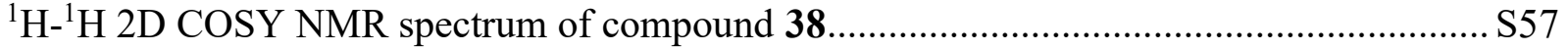

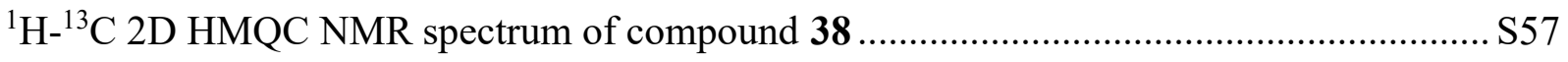

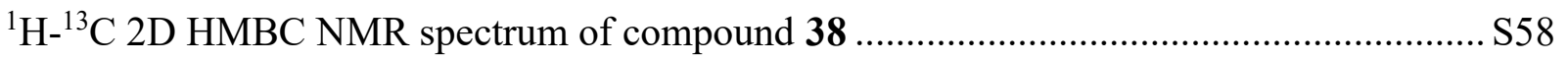

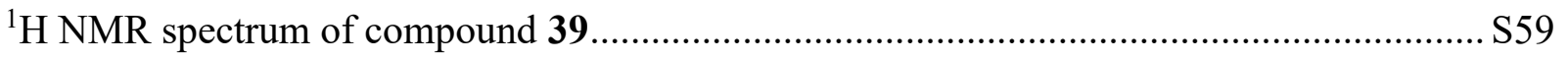

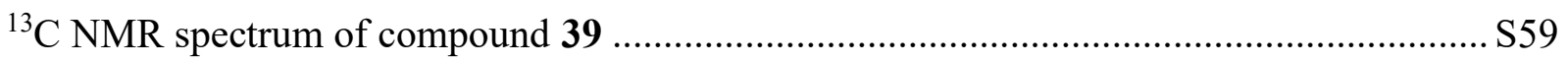

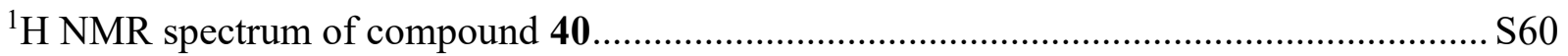

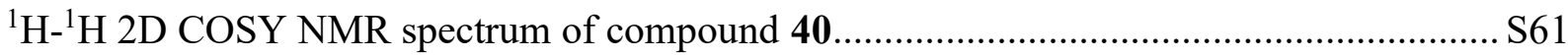

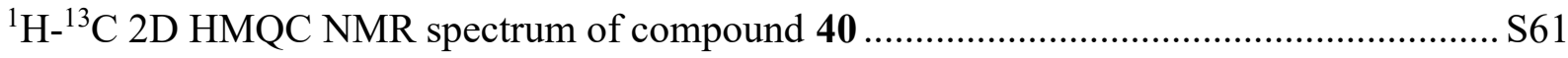

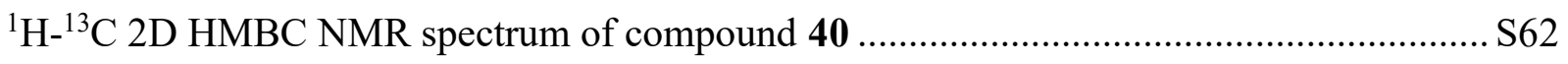

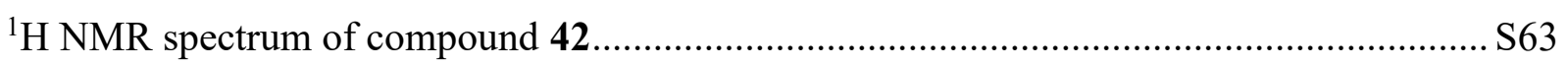

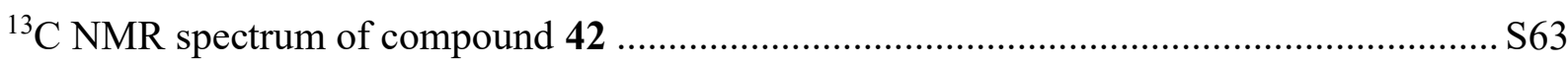

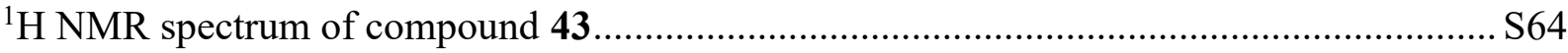

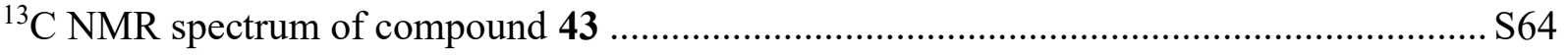

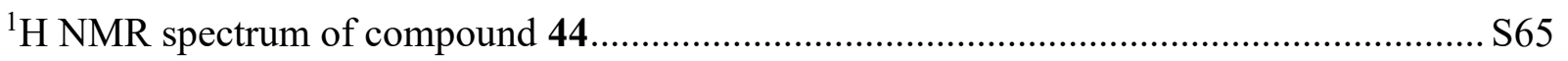




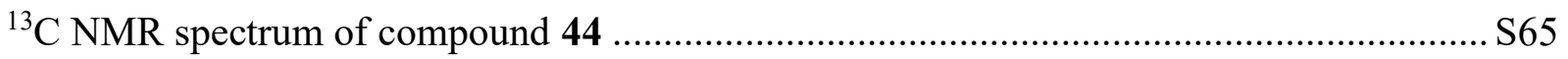

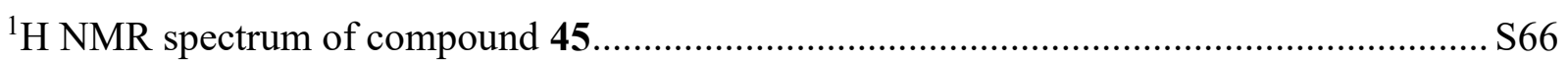

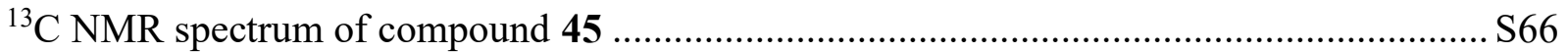

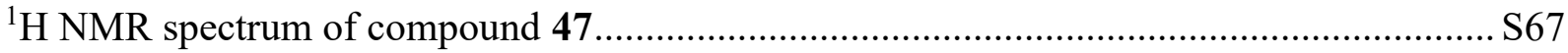

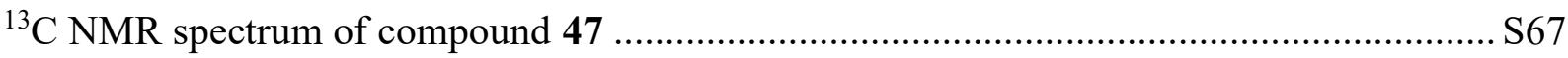

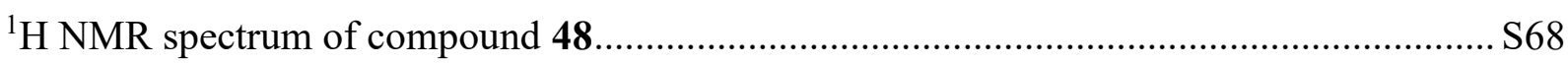

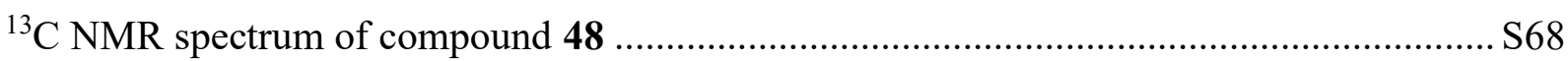

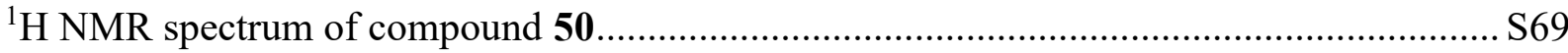

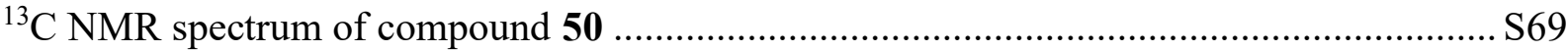

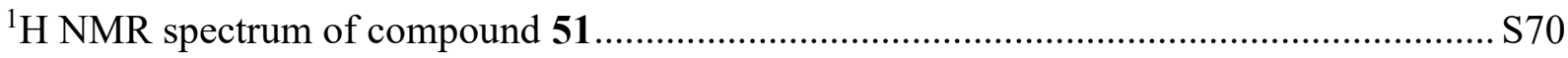

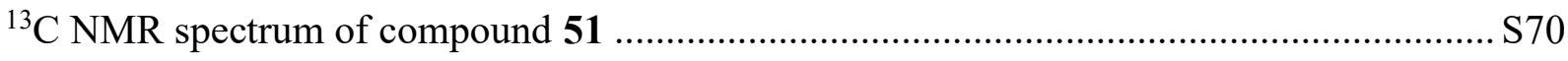

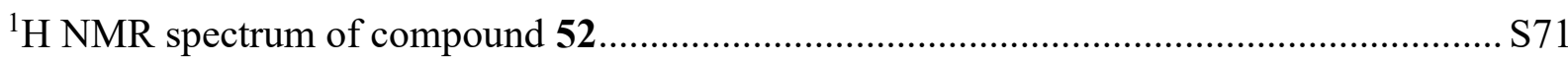

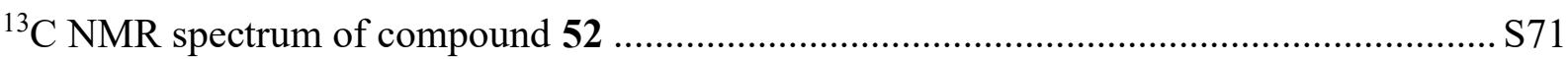

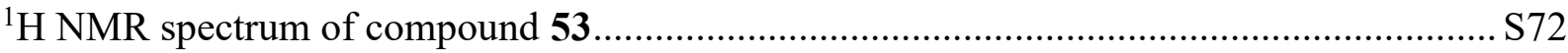

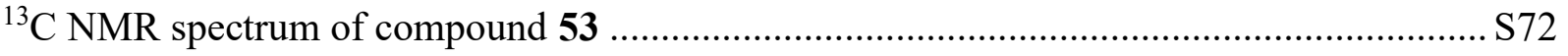


I. Supporting Schemes, Figures, and Tables

Scheme S1. Synthesis of Type I LacNAc Hexasaccharide 36 by [4 + 2] Glycosylation

Between Type I LacNAc Tetrasaccharide Donor 25 and Anomeric OTBS-Protected

Disaccharide Acceptor 35.

[4 + 2] Glycosylation
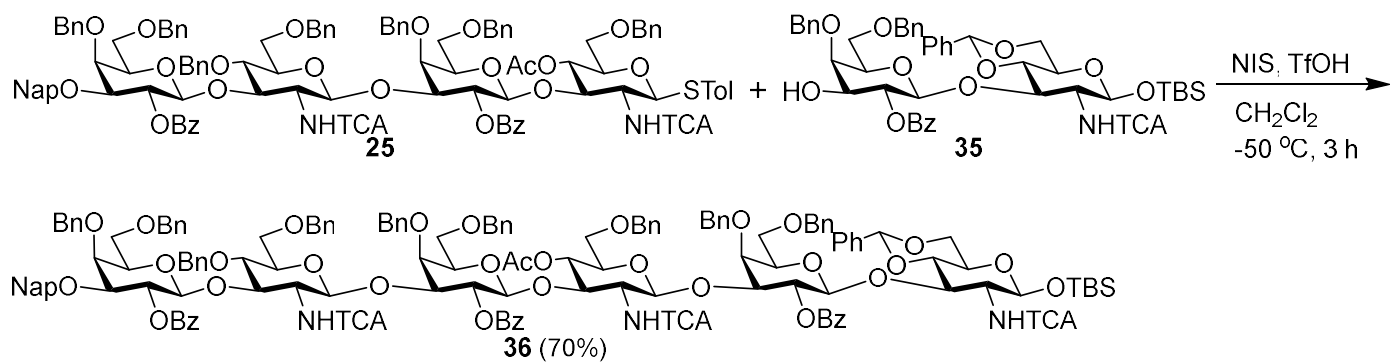

Scheme S2. Synthesis of Donor 45.
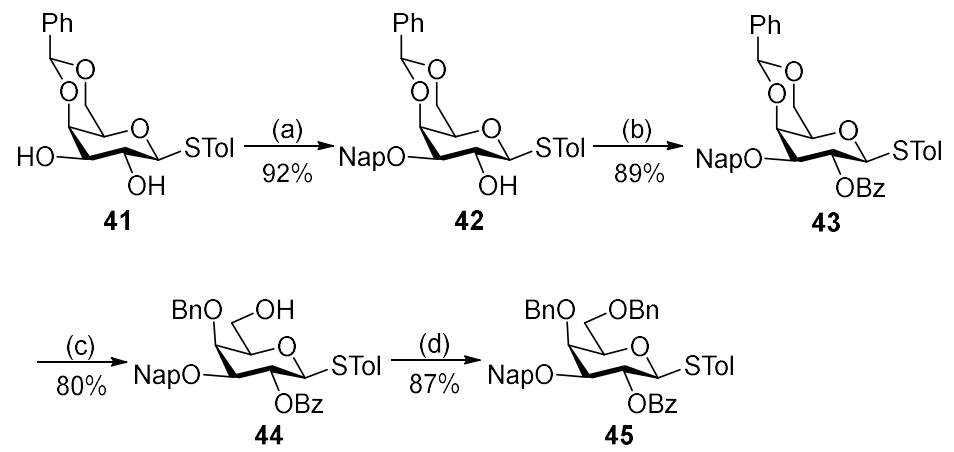

Reagents and conditions: (a) (i) $\mathrm{Bu}_{2} \mathrm{SnO}$, benzene, reflux overnight: (ii) $\mathrm{CsF}$ 2- $\mathrm{NapBr}$, DMF, rt, $6 \mathrm{~h}$;

(b) 4-DMAP, $\mathrm{Et}_{3} \mathrm{~N}, \mathrm{Bz}_{2} \mathrm{O}$, pyridine, rt, $3 \mathrm{~h}$; (c) $\mathrm{BH}_{3} \cdot \mathrm{THF}, \mathrm{TMSOTf}, \mathrm{CH}_{2} \mathrm{Cl}_{2}$,

$0{ }^{\circ} \mathrm{C}, 2 \mathrm{~h}$; (d) $\mathrm{NaH}, \mathrm{BnBr}, \mathrm{DMF}, 0{ }^{\circ} \mathrm{C}, 3 \mathrm{~h}$.

Scheme S3. Synthesis of GIcNTCA Building Block 48.

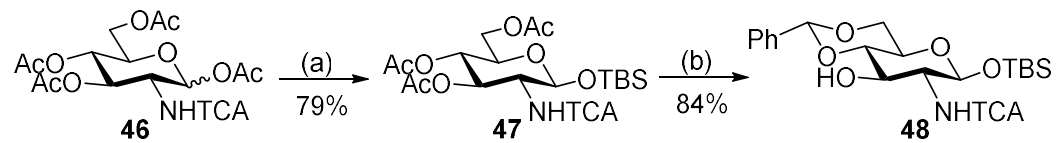

Reagents and conditions: (a) (i) $\mathrm{NH}_{2} \mathrm{NH}_{2} / \mathrm{AcOH}$, DMF, $1 \mathrm{~h}$; (ii) TBDMSCl, Imidazole, DMF, $2 \mathrm{~h}$;

(b) (i) $\mathrm{NaOMe}, \mathrm{CH}_{3} \mathrm{OH} / \mathrm{CH}_{2} \mathrm{Cl}_{2}, 1 \mathrm{~h}$; (ii) $\mathrm{PhCH}(\mathrm{OMe})_{2}, \mathrm{CSA}, \mathrm{CH}_{3} \mathrm{CN}, 3 \mathrm{~h}$. 
Scheme S4. Synthesis of Disaccharide Building Blocks 2 and 35.

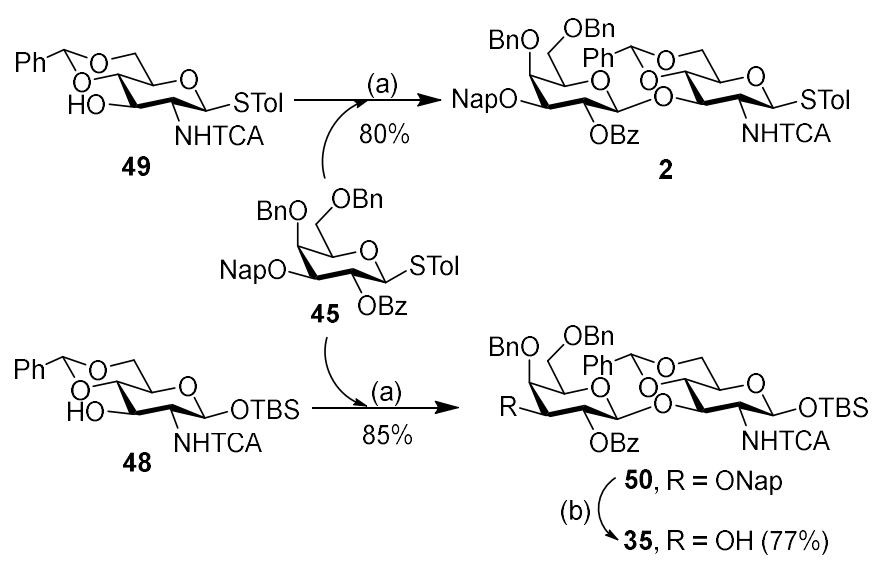

Reagents and conditions: (a) NIS, TMSOTf $-60^{\circ} \mathrm{C}: \mathrm{CH}_{2} \mathrm{Cl}_{2}, 4 \mathrm{~h}$;

(b) $\mathrm{DDQ}, \mathrm{CH}_{2} \mathrm{Cl}_{2} / \mathrm{H}_{2} \mathrm{O}, 0^{\circ} \mathrm{C}$ to rt, $6 \mathrm{~h}$

\section{Scheme S5. Synthesis of Several Type I LacNAc Disaccharide Donors (4, 6, 7, and 8) and Acceptors (10-13 and 15).}

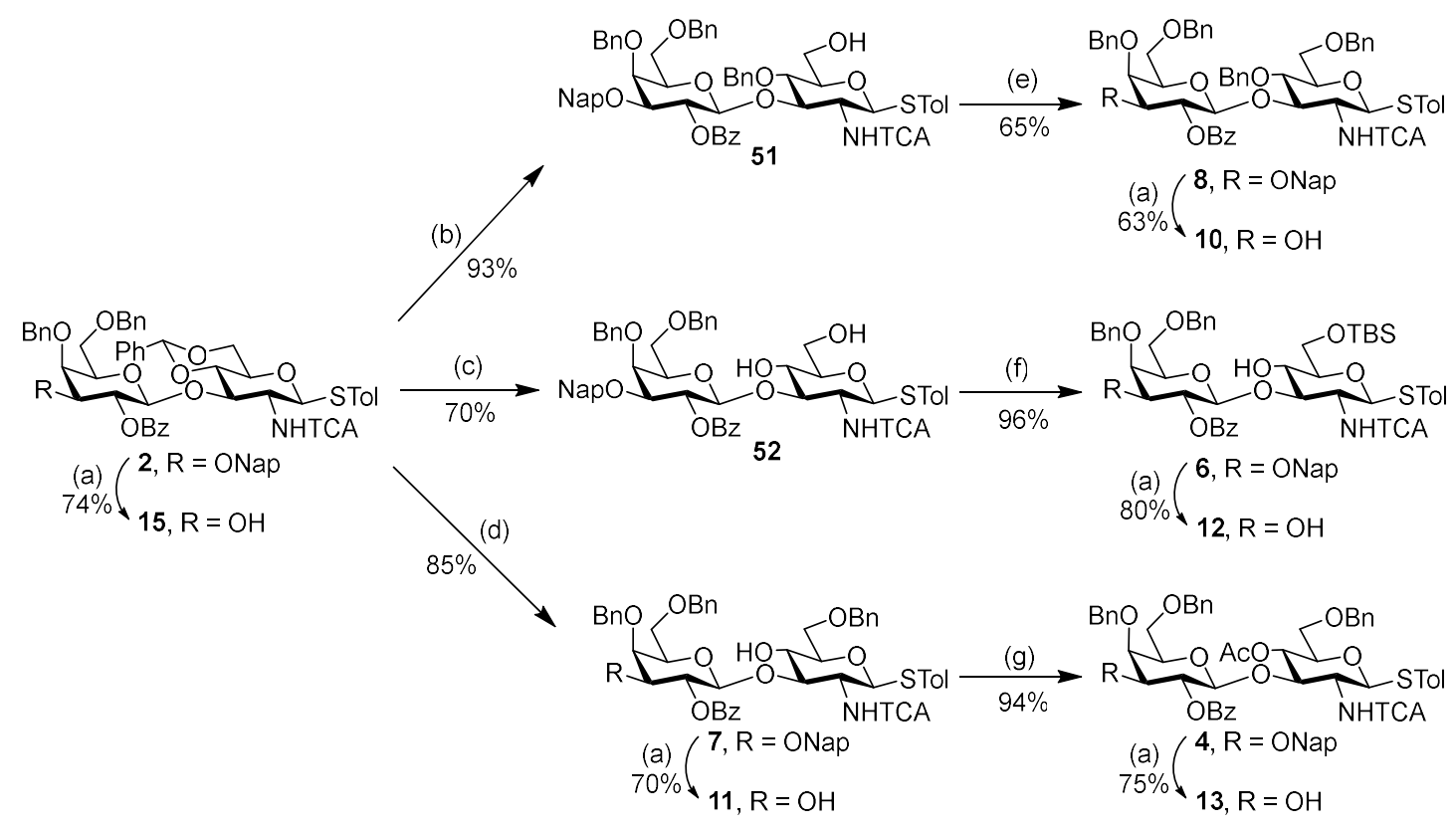

Reagents \& conditions: (a) DDQ, $\mathrm{CH}_{2} \mathrm{Cl}_{2} / \mathrm{PBS}: 10 / 1,0^{\circ} \mathrm{C}$ to rt, Argon; (b) $\mathrm{BH}_{3}$.THF, TMSOTf $\mathrm{CH}_{2} \mathrm{Cl}_{2}, 0^{\circ} \mathrm{C}$ to rt, $2 \mathrm{~h}$;

(c) aq. $\mathrm{AcOH}(80 \%), 54{ }^{\circ} \mathrm{C}, 3.5 \mathrm{~h}$; (d) $\mathrm{Et}_{3} \mathrm{SiH}$. TFAA. TFA, $\mathrm{CH}_{2} \mathrm{Cl}_{2}, \mathrm{O}^{\circ} \mathrm{C}$ to rt, $3 \mathrm{~h}$;

(e) DMF, TBAl $\mathrm{NaH}, \mathrm{BnBr}, 0^{\circ} \mathrm{C}$ to rt, $2 \mathrm{~h}$; (f) DMF, Imidazole, TBDMSCl, rt, $2.5 \mathrm{~h}$;

(g) 4-DMAP, $\mathrm{CH}_{2} \mathrm{Cl}_{2}, \mathrm{Et}_{3} \mathrm{~N}, \mathrm{Ac}_{2} \mathrm{O}, \mathrm{rt}, 2 \mathrm{~h}$ 
Scheme S6. Synthesis of Several Type I LacNAc Disaccharide Donors (1, 3, and 5) and Acceptors (14 and 16).

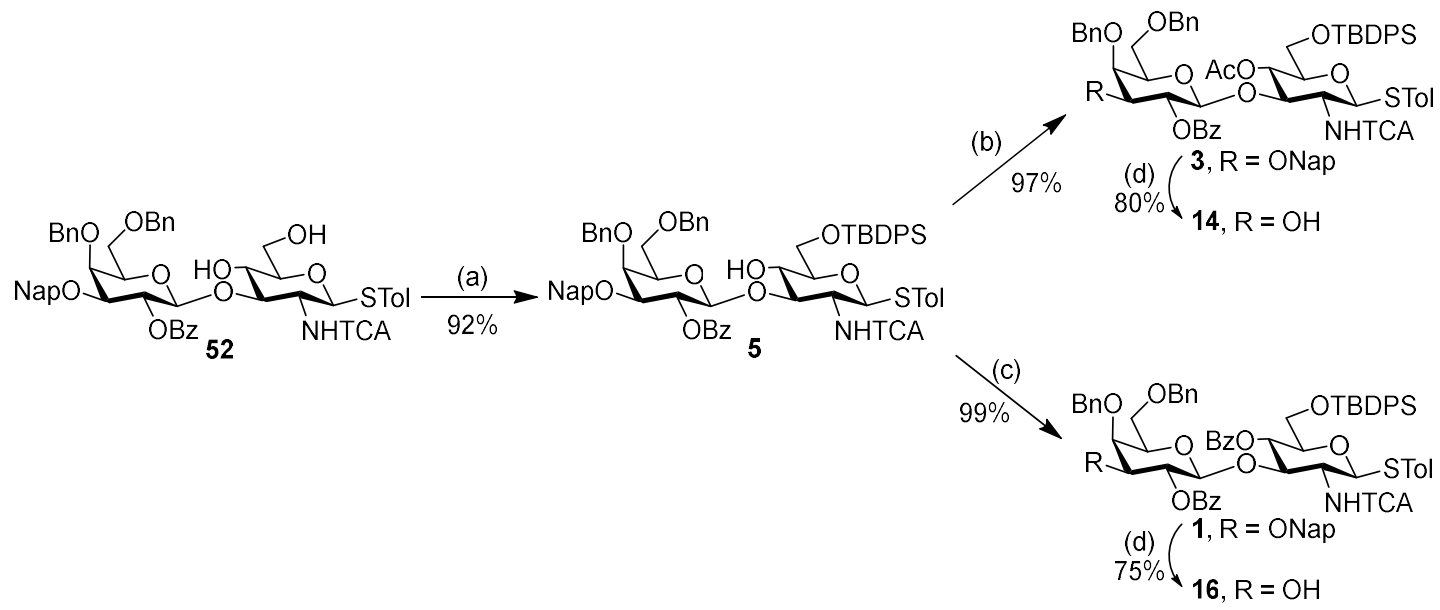

Reagents \& conditions: (a) DMF, Imidazole, TBDPSCl, rt, $1 \mathrm{~h}$; (b) 4-DMAP, $\mathrm{CH}_{2} \mathrm{Cl}_{2}, \mathrm{Et}_{3} \mathrm{~N}, \mathrm{Ac}_{2} \mathrm{O}, \mathrm{rt}, 1.5 \mathrm{~h}$;

(c) 4-DMAP, $\mathrm{CH}_{2} \mathrm{Cl}_{2}, \mathrm{Et}_{3} \mathrm{~N}, \mathrm{BzCl}$, rt, $1.5 \mathrm{~h}$; (d) $\mathrm{CH}_{2} \mathrm{Cl}_{2} / \mathrm{PBS}: 10 / 1, \mathrm{DDQ}, 0^{\circ} \mathrm{C}$ to rt, Argon

Scheme S7. Synthesis of Type I LacNAc Disaccharide Donor 9.

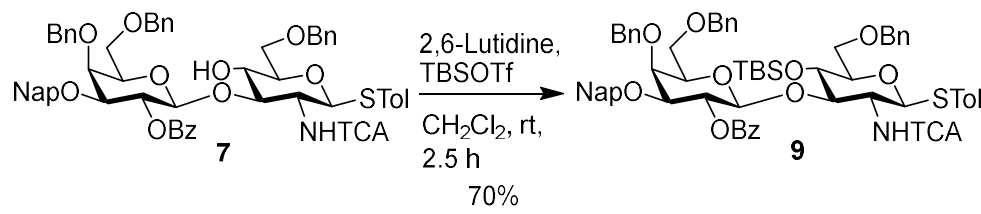

Scheme S8. $[2+2]$ Chemoselective Glycosylation by Using a Preactivation Method.

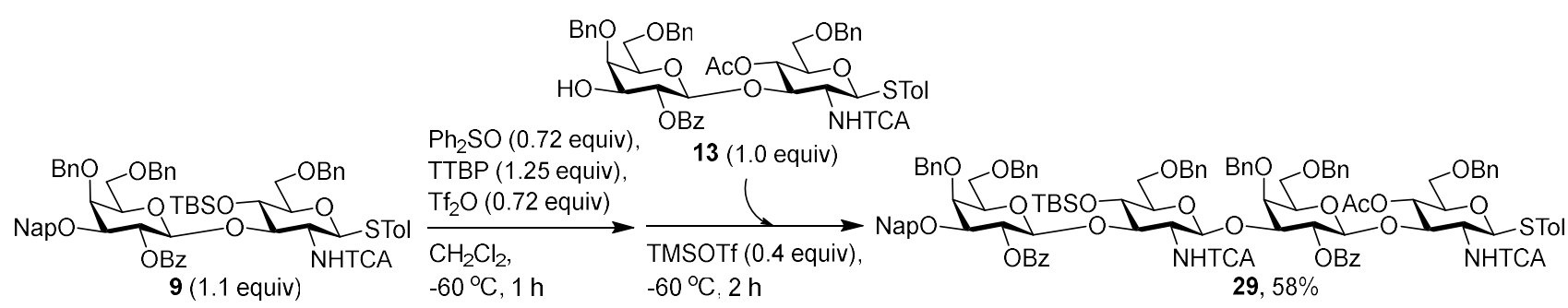




\section{Scheme S9. Synthesis of Type I LacNAc Tetrasaccharide Acceptor 37.}

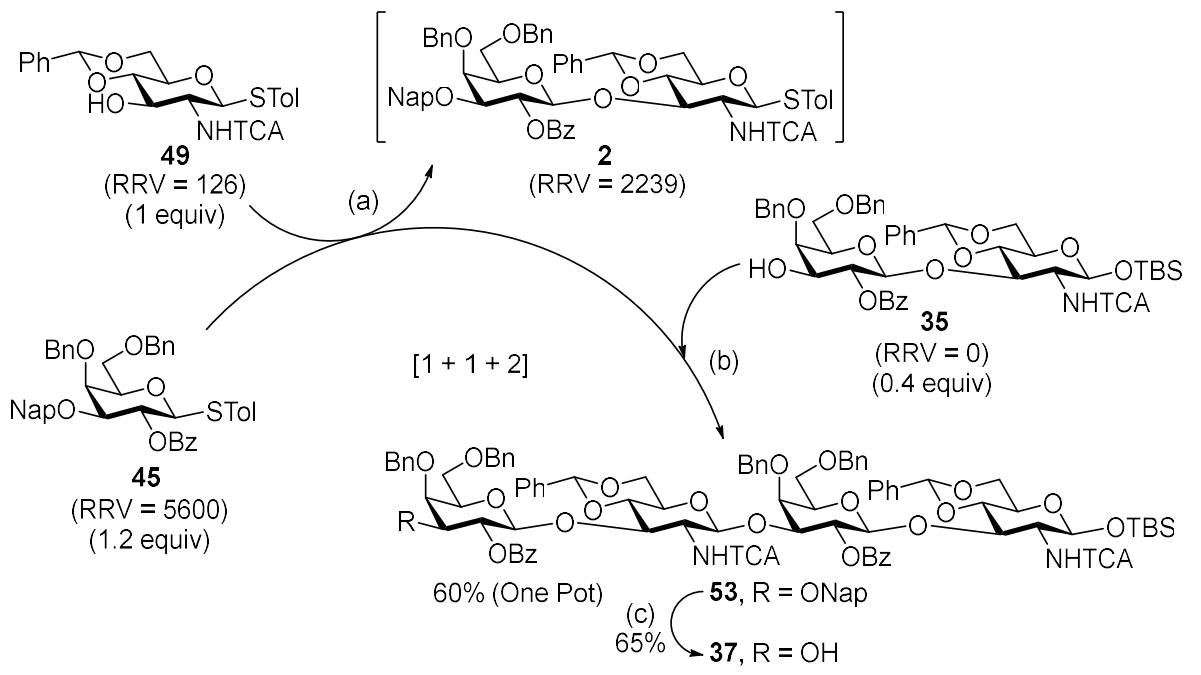

Reagents and conditions: (a) NIS ( 1.2 equiv), $\mathrm{TfOH}$ ( 0.2 equiv), $\mathrm{CH}_{2} \mathrm{Cl}_{2},-60{ }^{\circ} \mathrm{C}, 2 \mathrm{~h}$; (b) NIS (0.8 equiv)

$\mathrm{TfOH}\left(0.1\right.$ equiv), $-40^{\circ} \mathrm{C}, 12 \mathrm{~h}$; (c) $D D Q$ (3 equiv), $\mathrm{CH}_{2} \mathrm{Cl}_{2} / \mathrm{H}_{2} \mathrm{O}: 10 / 1(\mathrm{v} / \mathrm{v})$,

$\mathrm{O}^{\circ} \mathrm{C}$ to rt, $5 \mathrm{~h}$

Figure S1. Reference Donors Used for the RRV Determination.

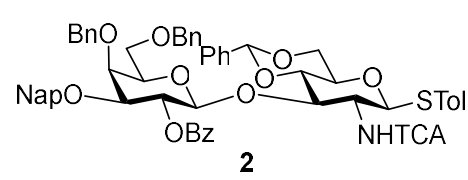

Reactivity ratio $=1.25$

$\mathrm{RRV}=2239$

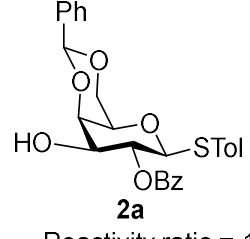

Reactivity ratio $=1$ RRV = 1791 (Ref)

Ref: Wong et al, J. Am. Chem. Soc. 1999, 121, 734-753

Compound 2a was used as a reference donor to determine the RRV of disaccharide 2. Reactivity Ratio of $\mathbf{2}=\left[\ln \left(\mathrm{A}_{2}\right)_{2 \mathrm{~h}}-\ln \left(\mathrm{A}_{2}\right)_{0}\right] /\left[\ln \left(\mathrm{A}_{2 \mathrm{a}}\right)_{2 \mathrm{~h}}-\ln \left(\mathrm{A}_{2 \mathrm{a}}\right)_{0}\right]$, which was determined by RRV experiment. 
Figure S2. Linear Correlation was Observed Between Estimated $\ln \left(R_{R} V_{D}\right)$ and Yield of Tetrasaccharide Products Shown in Table 1.

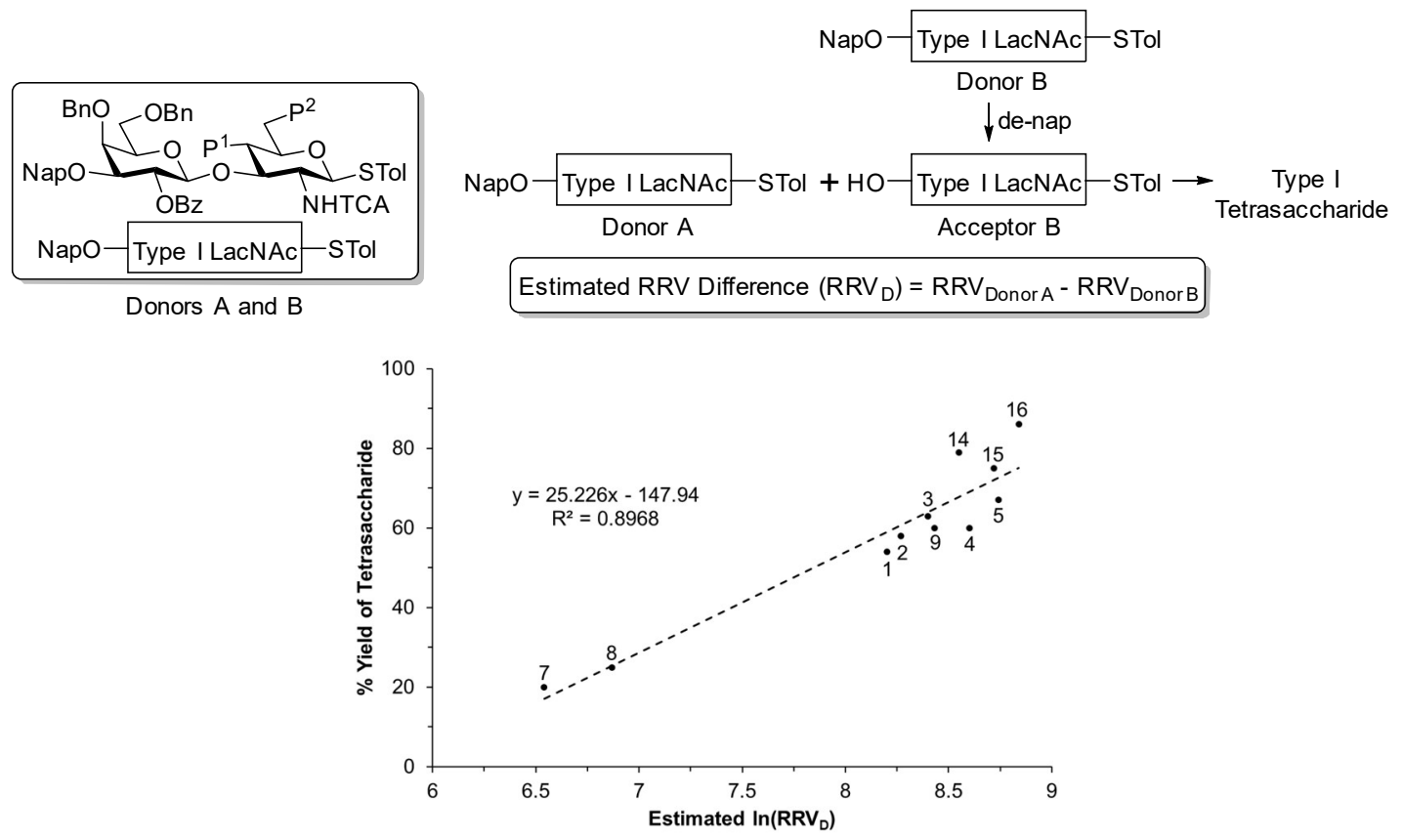

$\begin{array}{ccc}\begin{array}{c}\text { Estimated } \\ \ln \left(\mathrm{RRV}_{\mathrm{D}}\right)^{a}\end{array} & \begin{array}{c}\text { Estimated } \\ \mathrm{RRV}_{\mathrm{D}}\end{array} & \begin{array}{c}\text { yield } \\ (\%)\end{array} \\ <7.0 & <1097 & <25 \\ \sim 8.10-8.65 & 3294-5710 & \sim 50-70 \\ >8.8 & >6634 & >75\end{array}$

${ }^{a}$ Estimated $\mathrm{RRV}_{\mathrm{D}}=\mathrm{RRV}_{\text {Donor A }}-$

RRV Donor B

The dashed line was generated by fitting the data points that were performed under same conditions $\left(-50^{\circ} \mathrm{C}\right.$ and 1.3 equiv of donor) in Table 1 . Numbers next to data points represent the entry numbers in Table 1. Also see the Table S3. 
Table S1. Reactivity Ratio and RRV of Disaccharides 1-9.

Reactivity Ratio of any disaccharide $(\mathbf{X})=\left[\ln \left(\mathrm{Ax}_{\mathbf{x}}\right)_{2 \mathrm{~h}}-\ln \left(\mathrm{A}_{\mathbf{x}}\right)_{0}\right] /\left[\ln \left(\mathrm{A}_{\mathbf{2}}\right)_{2 \mathrm{~h}}-\ln \left(\mathrm{A}_{2}\right)_{0}\right]$, which was determined by RRV experiment.

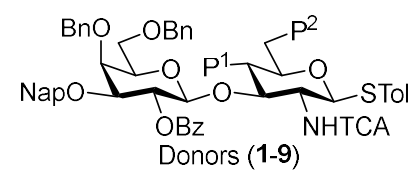

$\begin{array}{clccc}\text { Compd. } & \mathrm{P}^{1} & \mathrm{P}^{2} & \text { Reactivity Ratio } & \text { RRV } \\ \mathbf{1} & \text { OBz } & \text { OTBDPS } & 0.63 & 1411 \\ \mathbf{2} & \text { PhCH } & \text { (benzylidene) } & 1.0 & 2239 \\ \mathbf{3} & \text { OAc } & \text { OTBDPS } & 1.43 & 3202 \\ \mathbf{4} & \text { OAc } & \text { OBn } & 1.68 & 3762 \\ \mathbf{5} & \text { OH } & \text { OTBDPS } & 1.98 & 4433 \\ \mathbf{6} & \text { OH } & \text { OTBS } & 3.30 & 7389 \\ \mathbf{7} & \text { OH } & \text { OBn } & 3.42 & 7657 \\ \mathbf{8} & \text { OBn } & \text { OBn } & 3.73 & 8351 \\ \mathbf{9} & \text { OTBS } & \text { OBn } & 4.20 & 9404\end{array}$

Table S2. Reactivity Ratio and RRV of Disaccharides 10-16.

Reactivity Ratio of any disaccharide $(\mathbf{X})=\left[\ln (\mathrm{Ax})_{2} \mathrm{~h}-\ln (\mathrm{Ax})_{0}\right] /\left[\ln \left(\mathrm{A}_{2}\right)_{2} \mathrm{~h}-\ln \left(\mathrm{A}_{2}\right)_{0}\right]$, which was determined by RRV experiment.

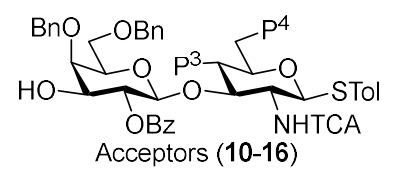

\begin{tabular}{|c|c|c|c|c|}
\hline Compd. & $\mathrm{P}^{3}$ & $\mathrm{P}^{4}$ & Reactivity Ratio & RRV \\
\hline 10 & OBn & OBn & 4.35 & 9740 \\
\hline 11 & $\mathrm{OH}$ & $\mathrm{OBn}$ & 2.43 & 5441 \\
\hline 12 & $\mathrm{OH}$ & OTBS & 2.38 & 5329 \\
\hline 13 & OAc & OBn & 1.29 & 2888 \\
\hline 14 & OAc & OTBDPS & 0.58 & 1299 \\
\hline 15 & \multicolumn{2}{|c|}{$\mathrm{PhCH}$ (benzylidene) } & 0.85 & 1903 \\
\hline 16 & $\mathrm{OBz}$ & OTBDPS & 0.92 & 2060 \\
\hline
\end{tabular}

Compound 2 was used as a reference donor to determine the RRVs of disaccharides $\mathbf{1}$ and 3-16. 
Table S3. Listed Estimated $\ln \left(R_{R} V_{D}\right)$ of the [2 + 2] Glycosylations that are Shown in Table 1.

See Figure $\mathrm{S} 2$ for the definition of estimated $\ln \left(\mathrm{RRV}_{\mathrm{D}}\right)$.

\begin{tabular}{|c|c|c|c|c|c|c|c|}
\hline entry & $\begin{array}{c}\text { Donor A } \\
\text { (equiv), RRV }\end{array}$ & $\begin{array}{c}\text { Acceptor B } \\
\text { (1 equiv), RRV }\end{array}$ & $\begin{array}{l}\text { Corresponding } \\
\text { Donor B, RRV }\end{array}$ & $\begin{array}{l}\text { Estimated } \\
\ln \left(R R V_{D}\right)^{a}\end{array}$ & $\begin{array}{l}\text { temp } \\
\left({ }^{\circ} \mathrm{C}\right)\end{array}$ & $\begin{array}{l}\text { time } \\
\text { (h) }\end{array}$ & $\begin{array}{c}\text { product } \\
(\% \text { yield })^{b}\end{array}$ \\
\hline 1 & $6(1.3), 7389$ & $\mathbf{1 3}, 2888$ & 4,3762 & 8.2 & -50 & 2.5 & $17(54)^{c}$ \\
\hline 2 & $7(1.3), 7657$ & $\mathbf{1 3}, 2888$ & 4,3762 & 8.27 & -50 & 2.5 & $18(58)$ \\
\hline 3 & $7(1.3), 7657$ & $\mathbf{1 4}, 1299$ & 3,3202 & 8.40 & -50 & 2.5 & $19(63)$ \\
\hline 4 & 7 (1.3), 7657 & $\mathbf{1 5}, 1903$ & 2,2239 & 8.60 & -50 & 2 & $20(60)$ \\
\hline 5 & $7(1.3), 7657$ & $\mathbf{1 6}, 2060$ & $\mathbf{1}, 1411$ & 8.74 & -50 & 2.5 & $21(67)$ \\
\hline 6 & $8(1.3), 8351$ & $\mathbf{1 0}, 9740$ & $\mathbf{8}, 8351$ & $-{ }^{d}$ & -50 & 2.5 & $22(19)^{e}$ \\
\hline 7 & $8(1.3), 8351$ & $\mathbf{1 1}, 5441$ & 7,7657 & 6.54 & -50 & 2.5 & $23(20)^{f}$ \\
\hline 8 & $8(1.3), 8351$ & $\mathbf{1 2}, 5329$ & 6,7389 & 6.87 & -50 & 2.5 & $24(25)^{g}$ \\
\hline 9 & $8(1.3), 8351$ & $\mathbf{1 3}, 2888$ & 4,3762 & 8.43 & -50 & 2.5 & $25(60)$ \\
\hline 10 & $8(1.3), 8351$ & $\mathbf{1 3}, 2888$ & 4,3762 & 8.43 & -70 & 21 & $25(72)^{h}$ \\
\hline 11 & $8(1.3), 8351$ & $\mathbf{1 3}, 2888$ & 4,3762 & 8.43 & -60 & 2.5 & $25(68)$ \\
\hline 12 & $8(1.1), 8351$ & $\mathbf{1 3}, 2888$ & 4,3762 & 8.43 & -60 & 2.5 & $25(63)$ \\
\hline 13 & $8(1.6), 8351$ & $\mathbf{1 3}, 2888$ & 4,3762 & 8.43 & -60 & 2.5 & $25(69)^{i}$ \\
\hline 14 & $8(1.3), 8351$ & $\mathbf{1 4}, 1299$ & 3,3202 & 8.55 & -50 & 2.5 & $26(79)$ \\
\hline 15 & $8(1.3), 8351$ & $\mathbf{1 5}, 1903$ & 2,2239 & 8.72 & -50 & 2 & $27(75)$ \\
\hline 16 & $\mathbf{8}(1.3), 8351$ & 16,2060 & $\mathbf{1}, 1411$ & 8.84 & -50 & 2.5 & $28(86)$ \\
\hline 17 & $8(1.1), 8351$ & 16, 2060 & $\mathbf{1}, 1411$ & 8.84 & -50 & 2.5 & $28(75)$ \\
\hline 18 & $\mathbf{9}(1.1), 9404$ & $\mathbf{1 3}, 2888$ & 4,3762 & 8.64 & -60 & 2.5 & $29(65)$ \\
\hline 19 & $\mathbf{9}(1.1), 9404$ & $\mathbf{1 3}, 2888$ & 4,3762 & 8.64 & -70 & 21 & $29(61)^{h}$ \\
\hline 20 & $9(1.1), 9404$ & 16,2060 & $\mathbf{1}, 1411$ & 8.99 & -60 & 4.5 & $30(52)^{j}$ \\
\hline
\end{tabular}

Glycosylation conditions: NIS (same as donor equiv), TMSOTf (0.2 equiv), $3 \AA \mathrm{MS}, \mathrm{CH}_{2} \mathrm{Cl}_{2}(50 \mathrm{mM}) .{ }^{a} \mathrm{RRV}_{\mathrm{D}}=$ $\mathrm{RRV}_{\text {Donor A }}-\mathrm{RRV}_{\text {Donor B. }}$ Also see Figure $\mathrm{S} 2$ for the detail. ${ }^{b}$ Isolated yield. ${ }^{c} 18 \%$ Acceptor recovered. ${ }^{d} \ln \left(\mathrm{RRV}_{\mathrm{D}}\right)$ for this entry is not valid, as $\mathrm{RRV}_{\mathrm{D}}$ is a negative number. ${ }^{e} 34 \%,{ }_{33} \%,{ }_{3} 37 \%$ Donor recovered respectively. ${ }^{h}$ Acceptor 13 remained after $2.5 \mathrm{~h}$ (entry 10)/2 h (entry 19), hence, the reactions were continued for longer time. ${ }^{i} 14 \%$ Oxazoline recovered. ${ }^{j}$ Donor spot disappeared with $1 / 3^{\text {rd }}$ acceptor remained unreacted (based on TLC). 


\section{NMR Spectra}

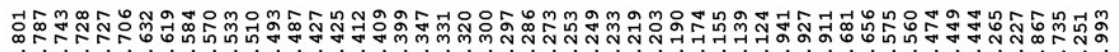

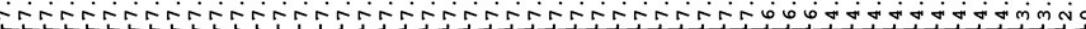

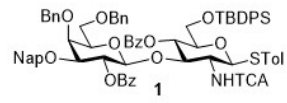

Mh will th
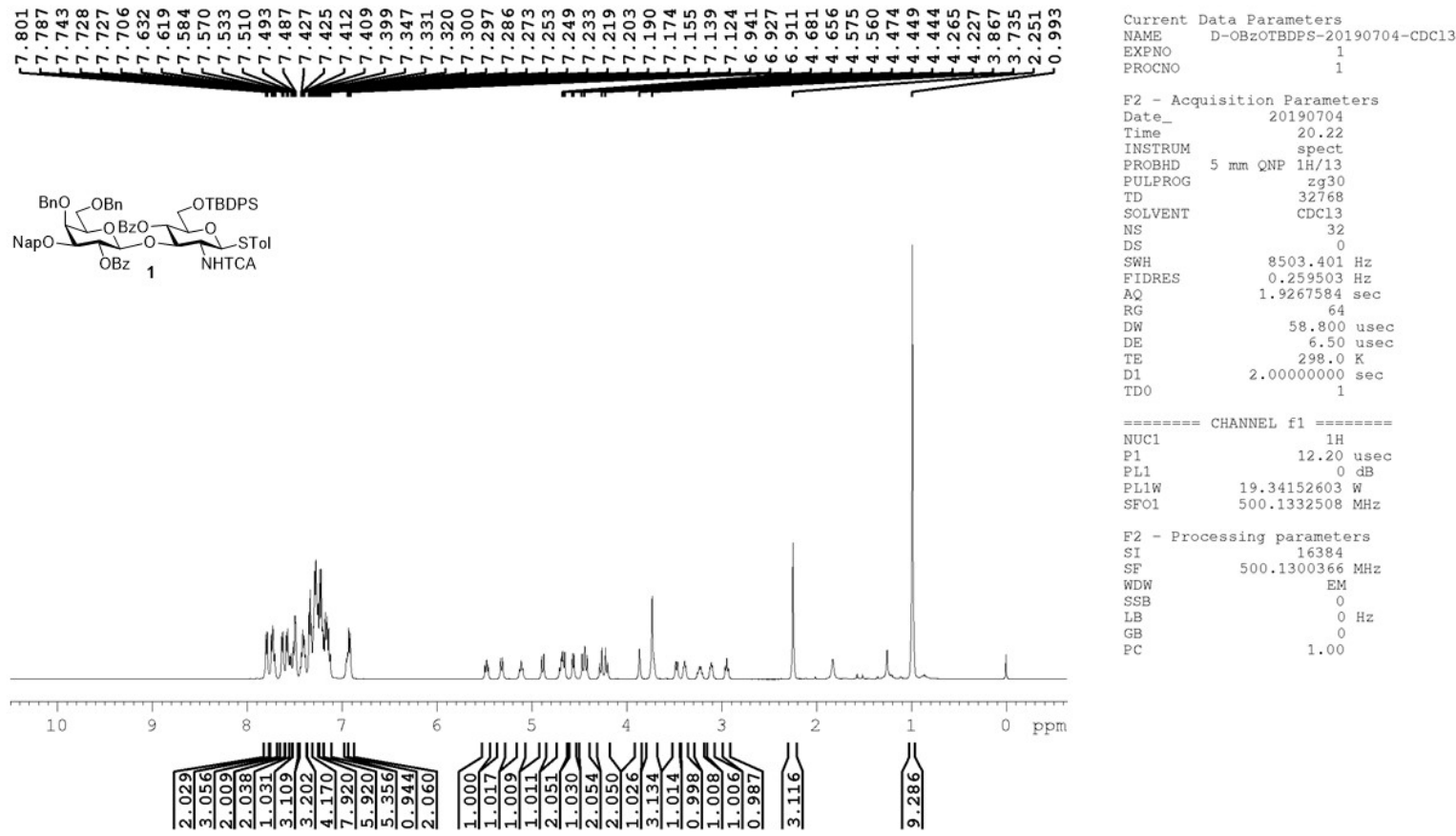

${ }^{1} \mathrm{H}$ NMR spectrum of compound $1\left(500 \mathrm{MHz}, \mathrm{CDCl}_{3}\right)$

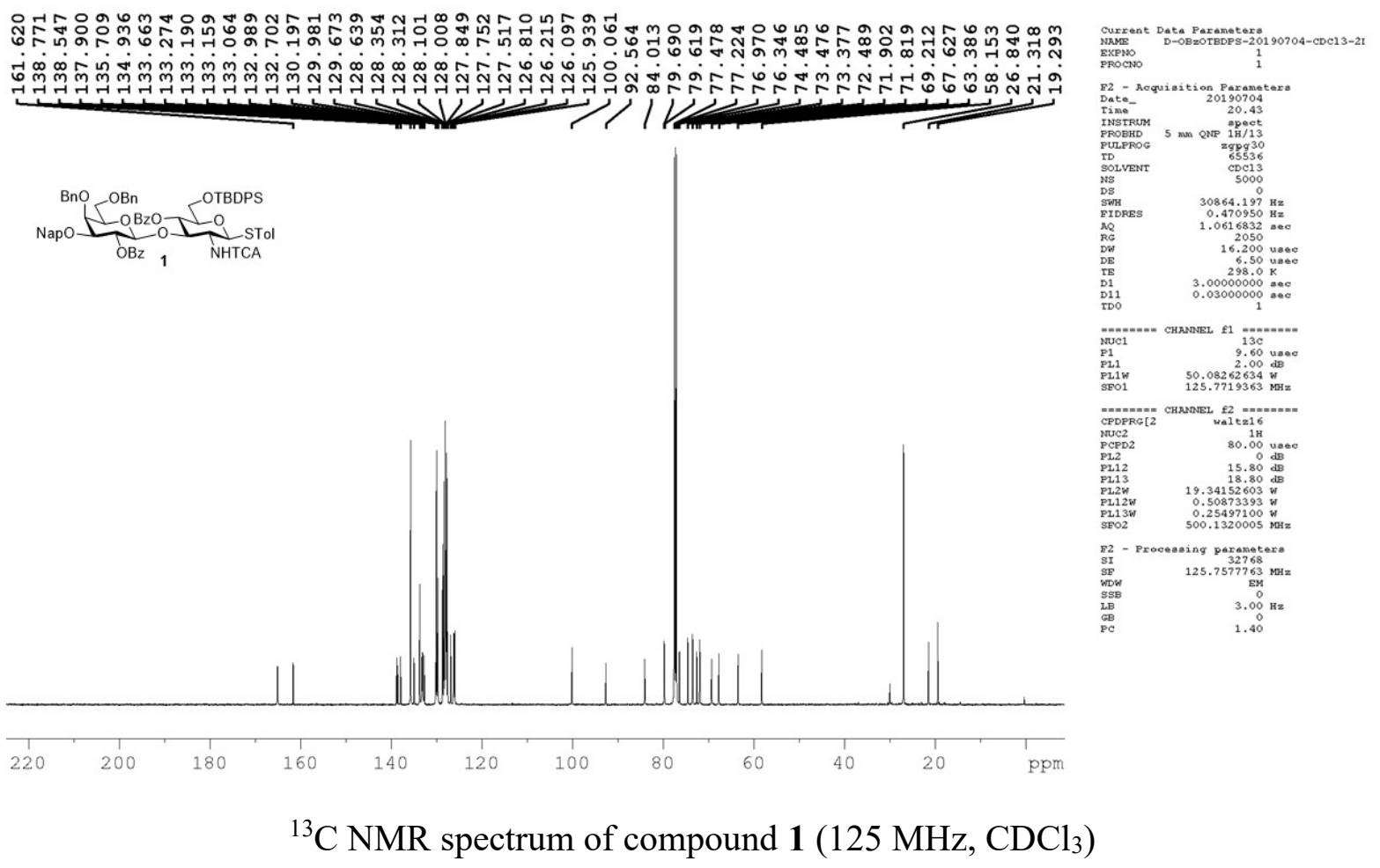




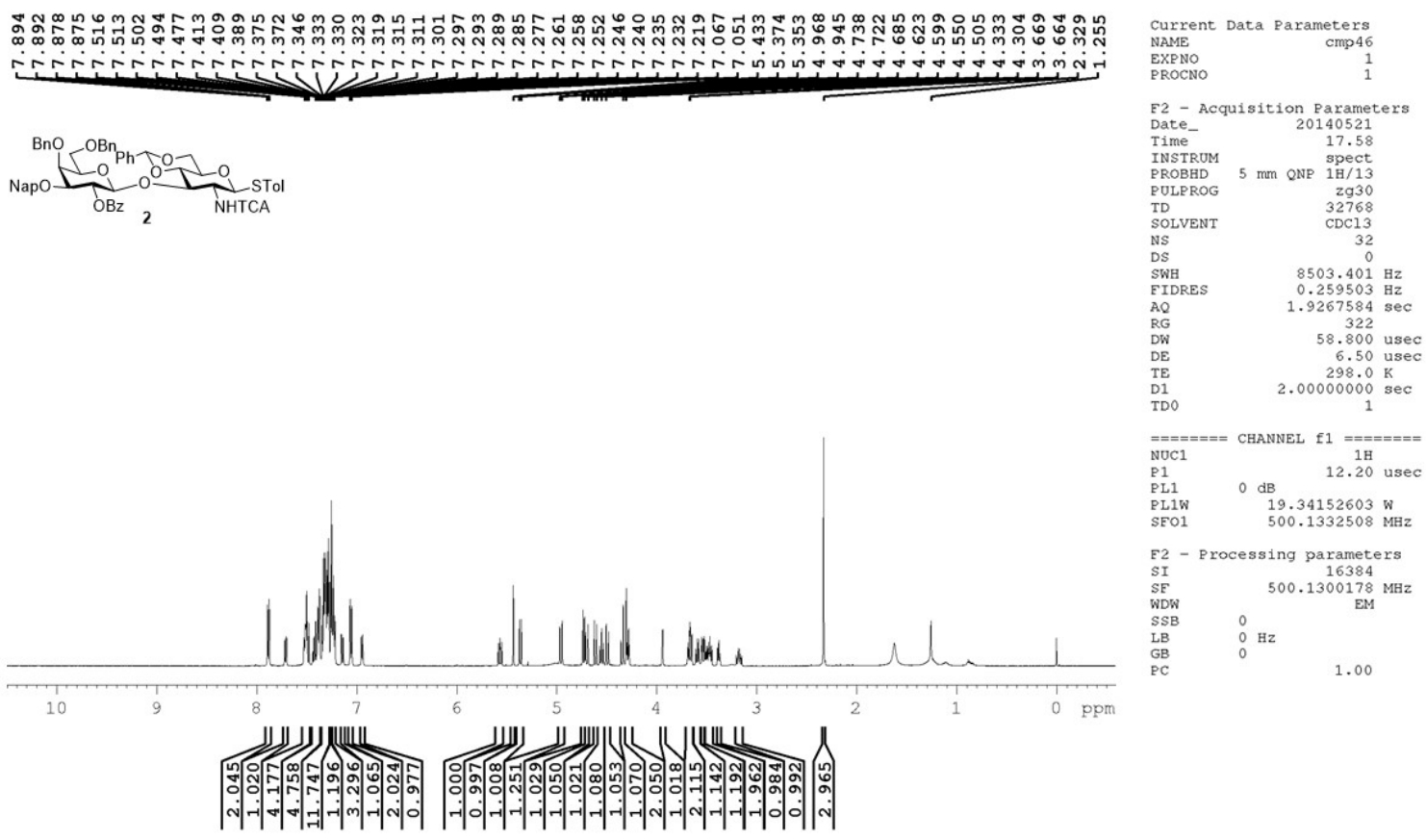

${ }^{1} \mathrm{H}$ NMR spectrum of compound $2\left(500 \mathrm{MHz}, \mathrm{CDCl}_{3}\right)$

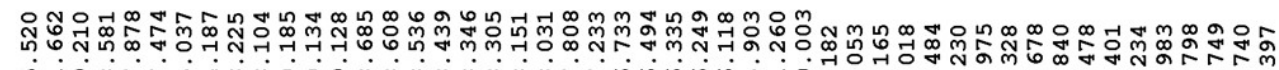

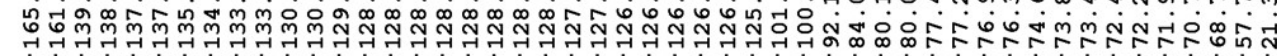

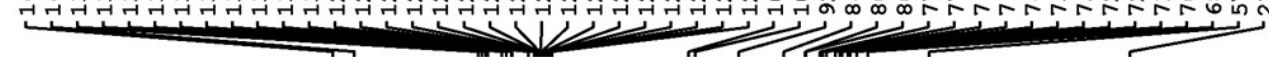

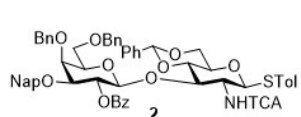
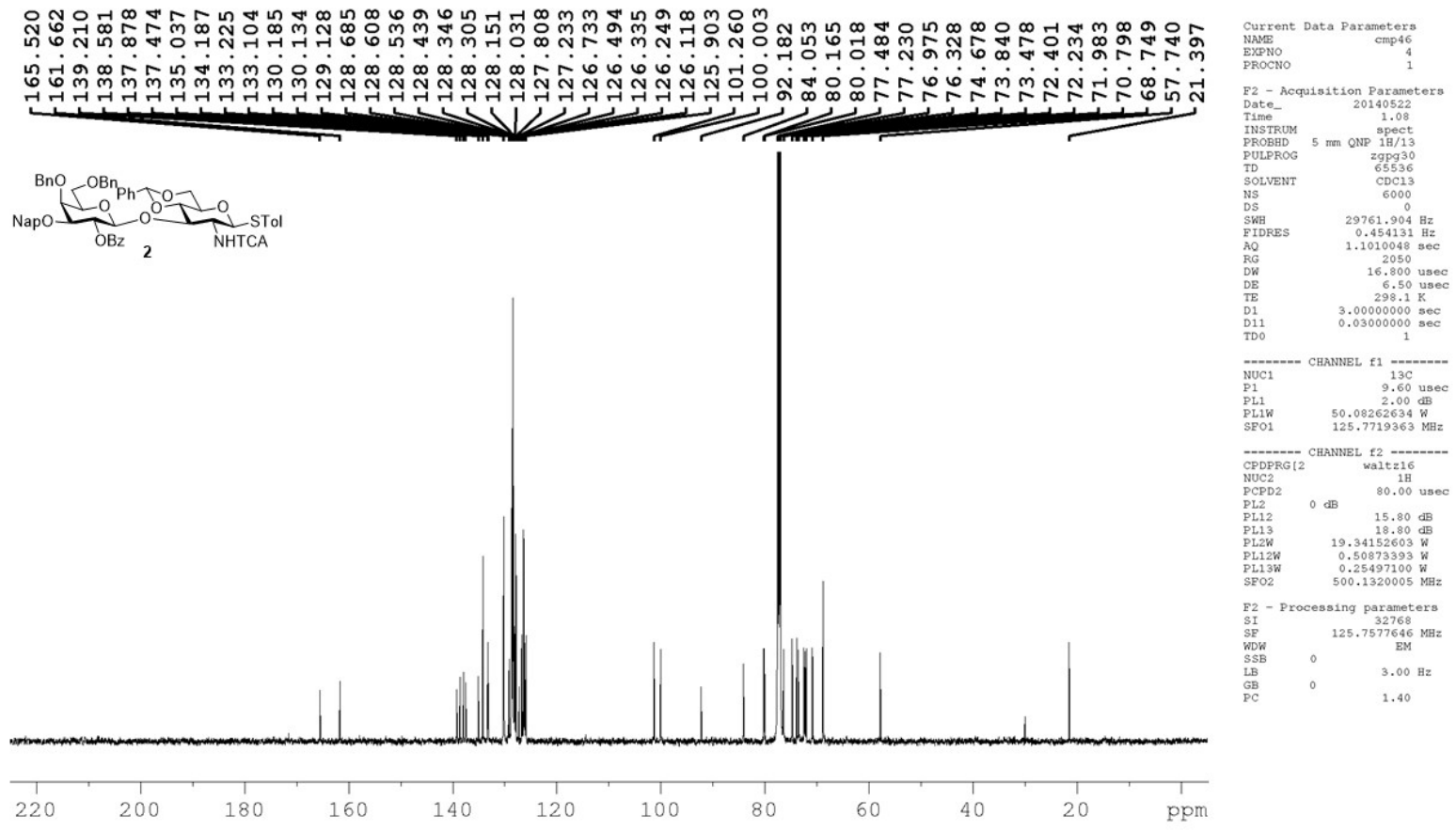

${ }^{13} \mathrm{C}$ NMR spectrum of compound $2\left(125 \mathrm{MHz}, \mathrm{CDCl}_{3}\right)$ 


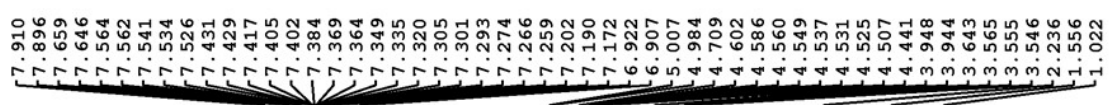

OBz ${ }_{3}^{\text {NHTCA }}$

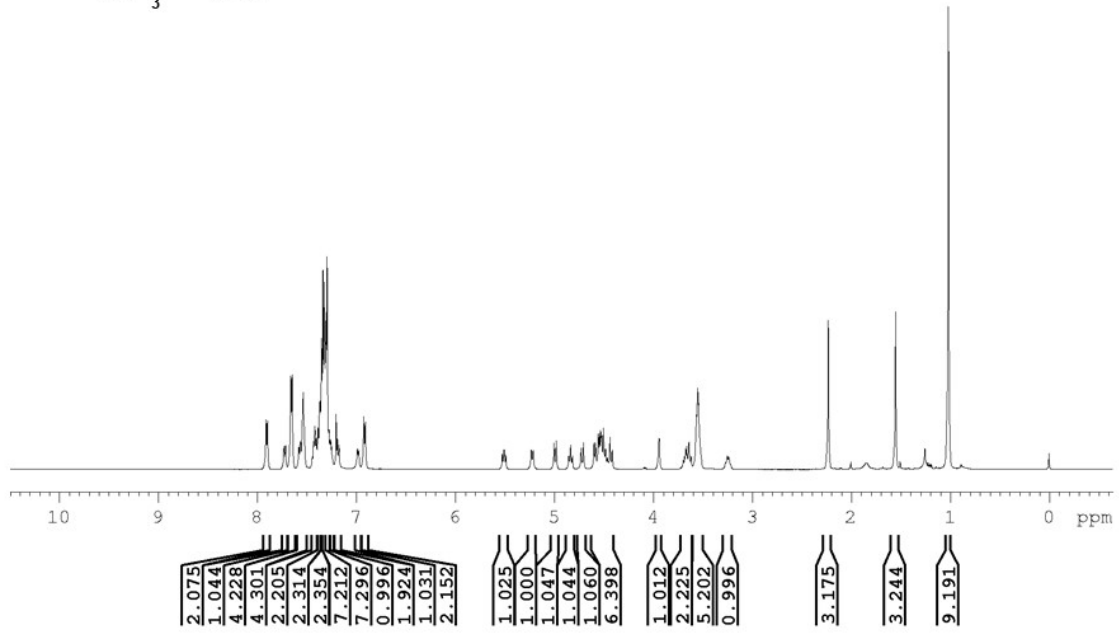

${ }^{1} \mathrm{H}$ NMR spectrum of compound $3\left(500 \mathrm{MHz}, \mathrm{CDCl}_{3}\right)$

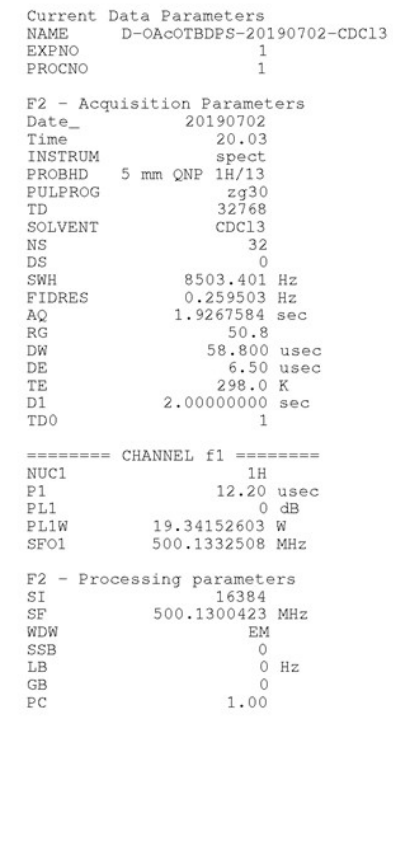

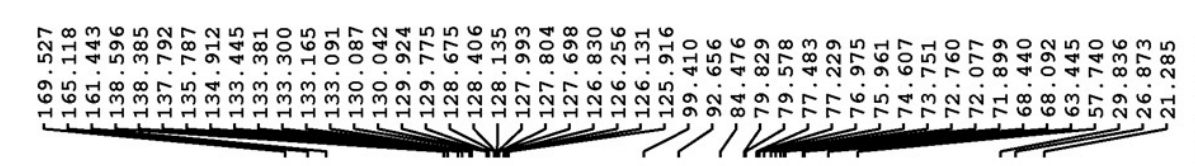
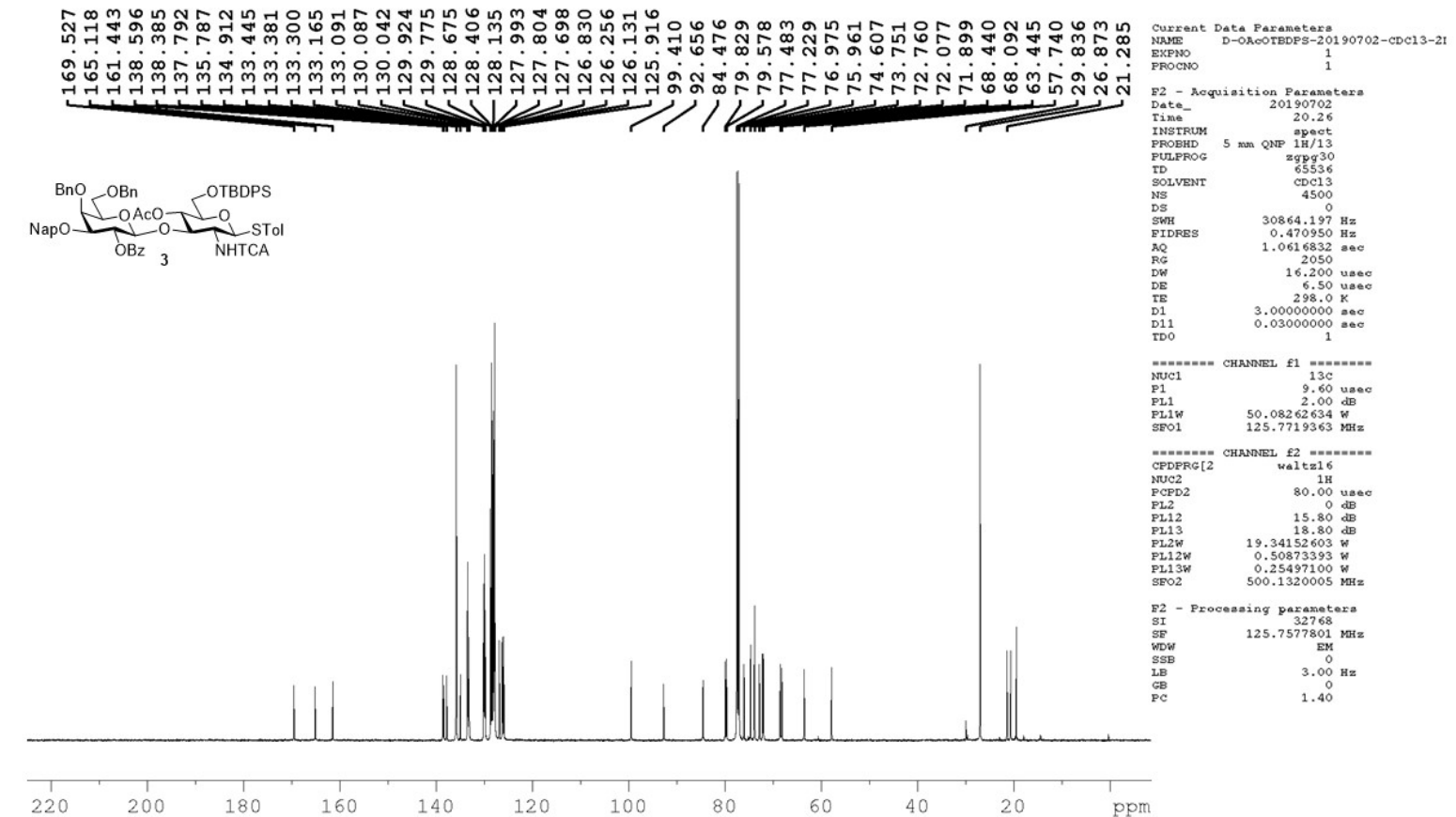

${ }^{13} \mathrm{C}$ NMR spectrum of compound $3\left(125 \mathrm{MHz}, \mathrm{CDCl}_{3}\right)$ 


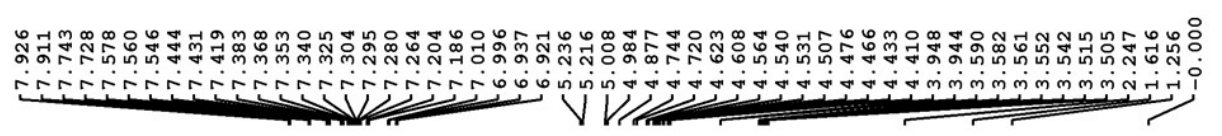

$\underbrace{}_{\mathrm{OBz}}{ }_{\mathrm{NHTCA}}^{\mathrm{OBn}}$

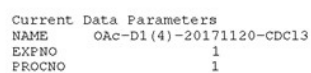

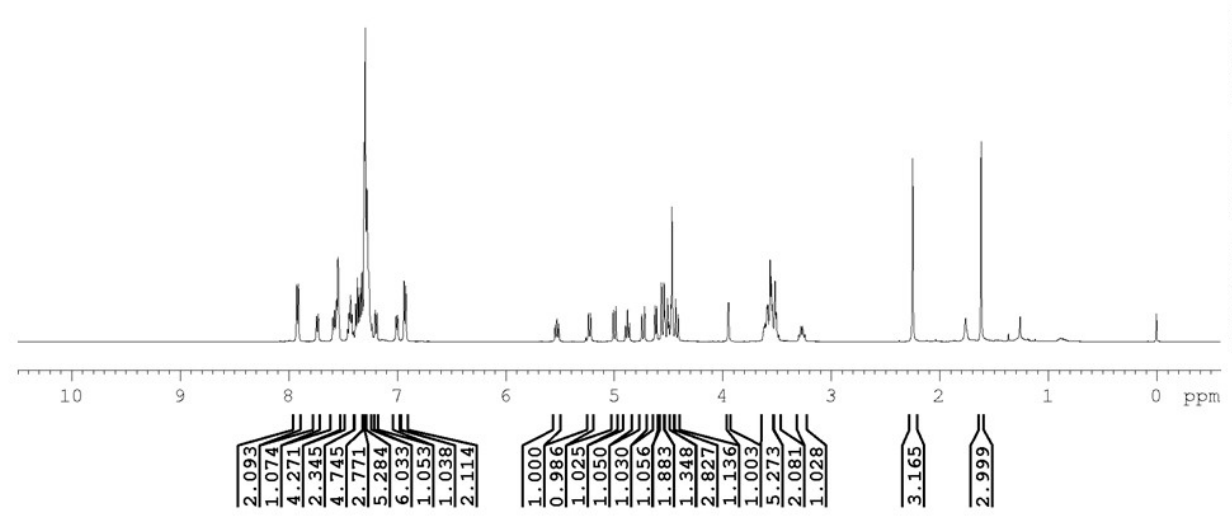

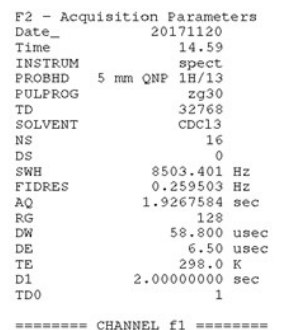

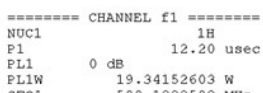

$\begin{array}{ll}\text { PL1W } & 19.34152603 \mathrm{~W} \\ \text { SFO1 } & 500.1332508 \mathrm{MHz}\end{array}$

F2 - Processing parameters
SI
16384
$500.1300275 \mathrm{MHz}$

$\begin{array}{llr}\text { SE } & & 500.1300275 \mathrm{MHz} \\ \text { WDW } & & \text { EM } \\ \text { SSB } & 0 & \\ \text { LB } & 0 & \\ \text { GB } & 0 & \\ \text { PC } & & \\ \text { PC } & & \end{array}$

${ }^{1} \mathrm{H}$ NMR spectrum of compound $4\left(500 \mathrm{MHz}, \mathrm{CDCl}_{3}\right)$

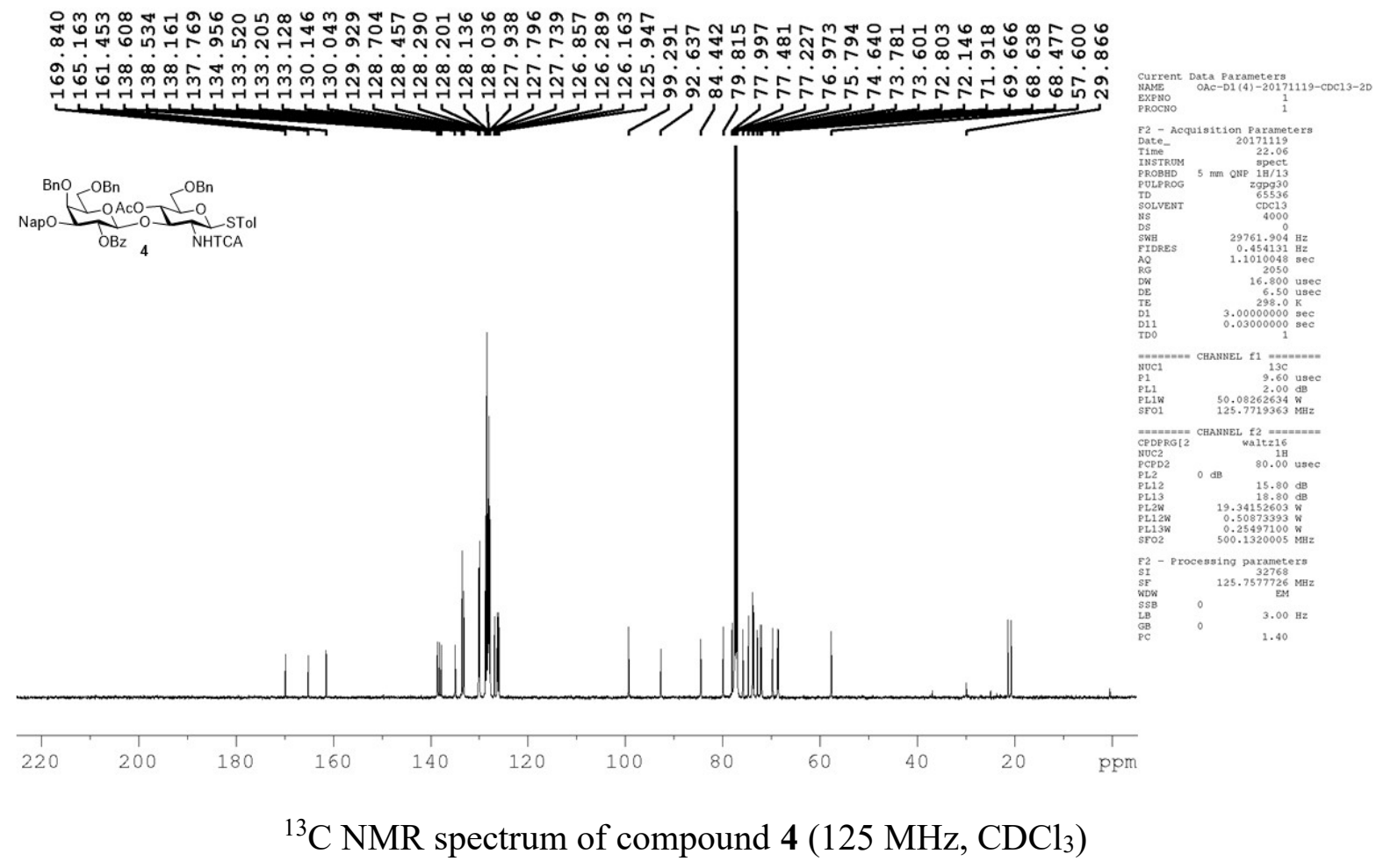




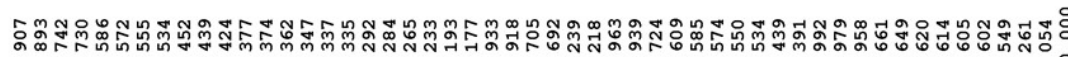

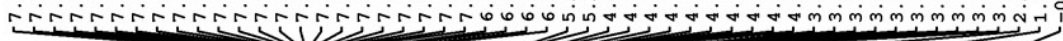

NapO

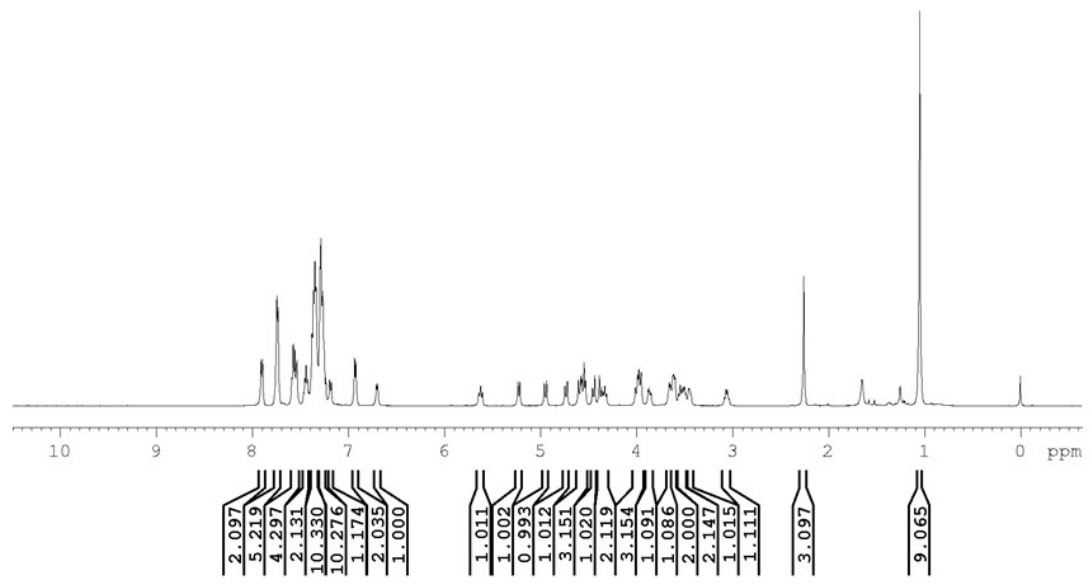

${ }^{1} \mathrm{H}$ NMR spectrum of compound $5\left(500 \mathrm{MHz}, \mathrm{CDCl}_{3}\right)$
Current Data Parameters EXPNO

F2 - Acquisition Parameters

Date_r 2019071

Time

PROBHD $5 \mathrm{~mm}$ QNP $\begin{aligned} & \text { spect } \\ & 1 \mathrm{H} / 13\end{aligned}$

TD

SOLV
NS

$\begin{array}{ll}\text { DS } & 8503.401 \mathrm{~Hz} \\ \text { SWH } & 0.259503 \mathrm{~Hz}\end{array}$

AQ $\quad 1.926758$

58.800 usec
DE
DE
TE

D1 $2.00000000 \mathrm{sec}$

$=======$ CHANNEL $\mathrm{fl} 1== \pm====$

$\begin{array}{lrl}\text { NuC1 } & 1 \mathrm{H} \\ \text { P1 } & 12.20 \text { usec } \\ \text { PL1 } & 0 \text { dB }\end{array}$

$\begin{array}{ll}\text { PL1W } & 19.34152603 \mathrm{~W} \\ \text { SEO1 } & 500.1332508 \mathrm{MHz}\end{array}$

F2 - Processing parameters

$\begin{array}{lr}\mathrm{SI} & 16384 \\ \mathrm{SF} & 500.1300268 \mathrm{MH}\end{array}$

$\mathrm{SSB}$
$\mathrm{LB}$
$\mathrm{GB}$
$\mathrm{PC}$

500.1300268

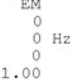

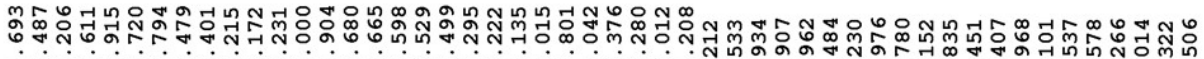

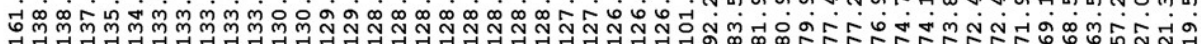

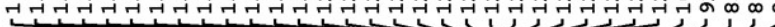
NapO $\underbrace{\mathrm{O}}_{\mathrm{OBz}} \underbrace{\mathrm{OBn}}_{5} \underbrace{\mathrm{OTBDPS}}_{\mathrm{NHTCA}}$
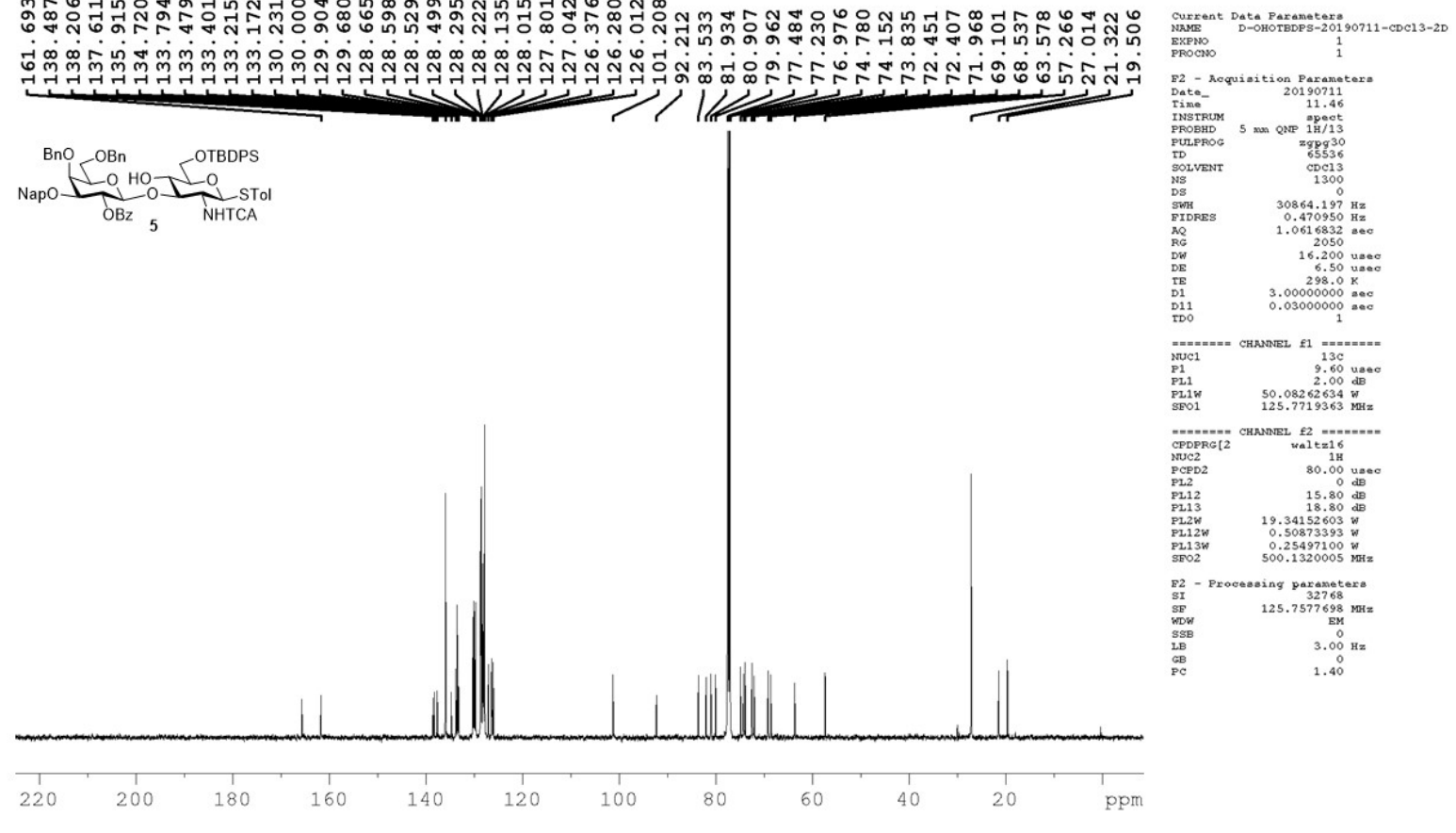

${ }^{13} \mathrm{C}$ NMR spectrum of compound $5\left(125 \mathrm{MHz}, \mathrm{CDCl}_{3}\right)$ 

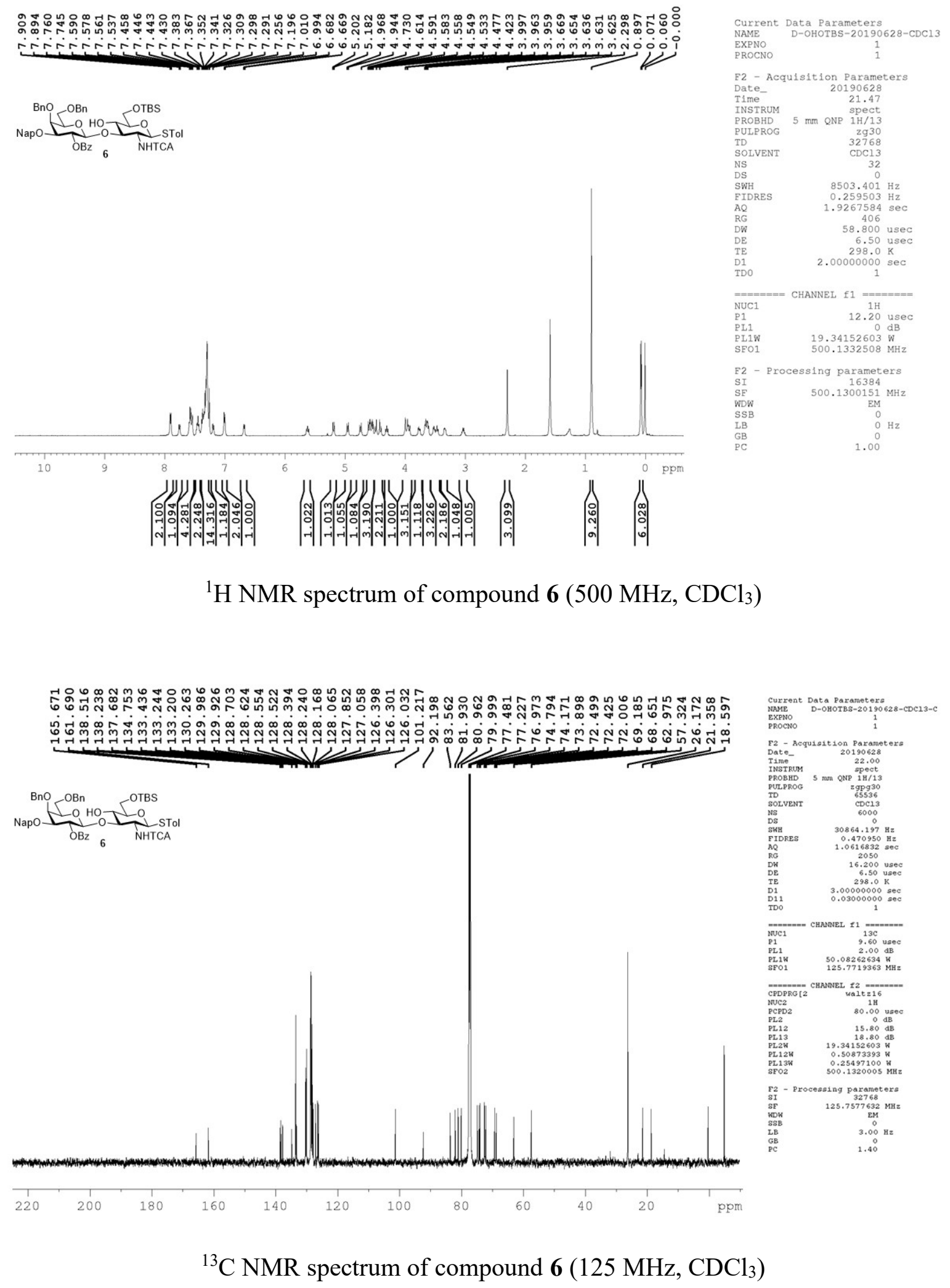

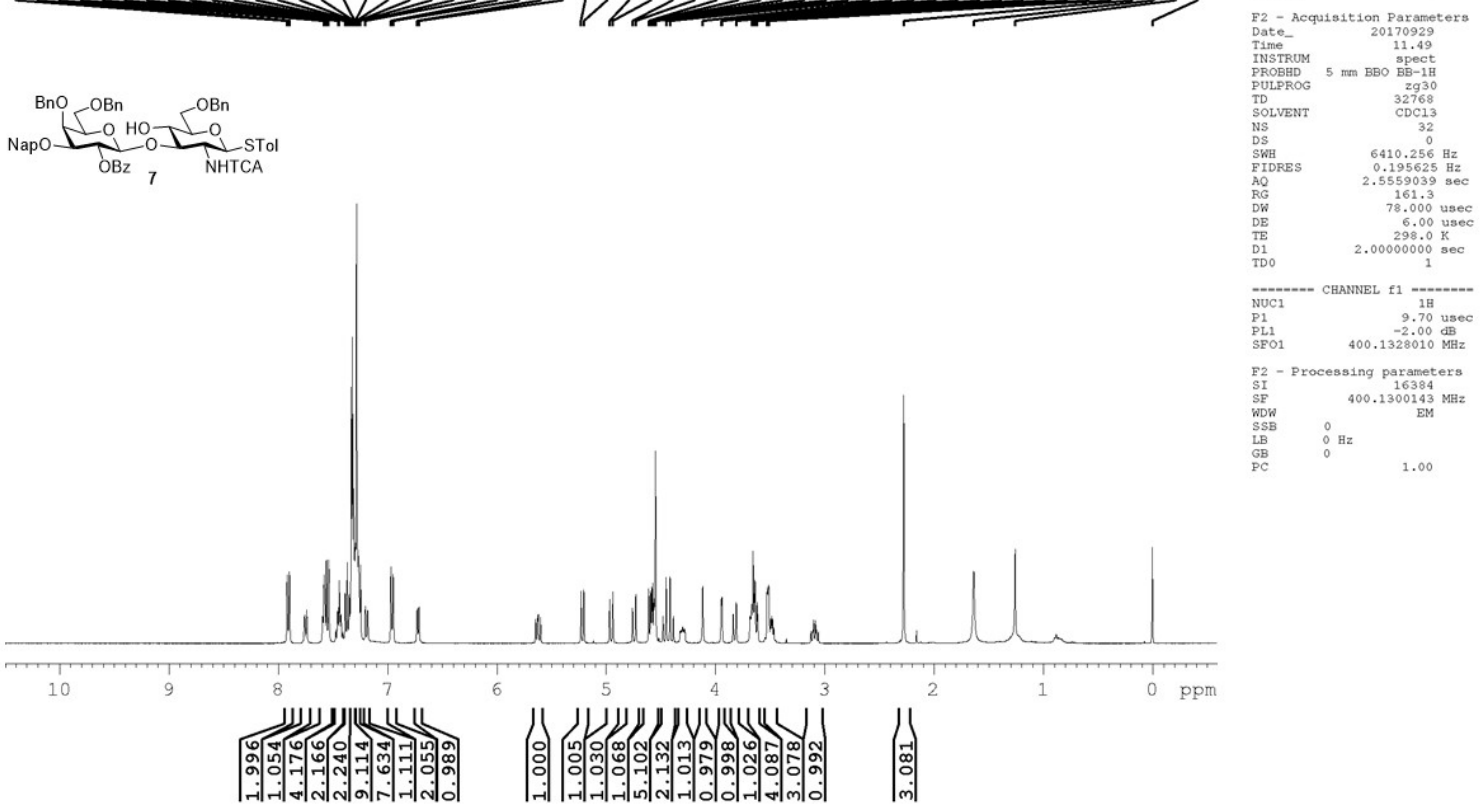

${ }^{1} \mathrm{H}$ NMR spectrum of compound $7\left(400 \mathrm{MHz}, \mathrm{CDCl}_{3}\right)$

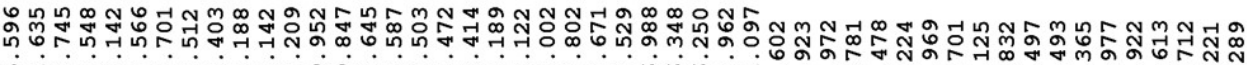

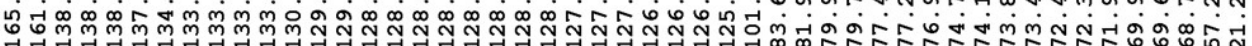

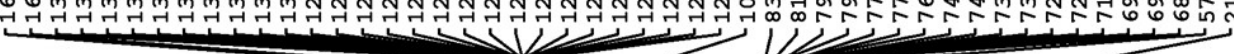
${ }_{\mathrm{OBz}}^{\mathrm{O}}{ }_{7}^{\mathrm{OBnO}} \underbrace{\mathrm{OBn}}_{\mathrm{NHTCA}}$
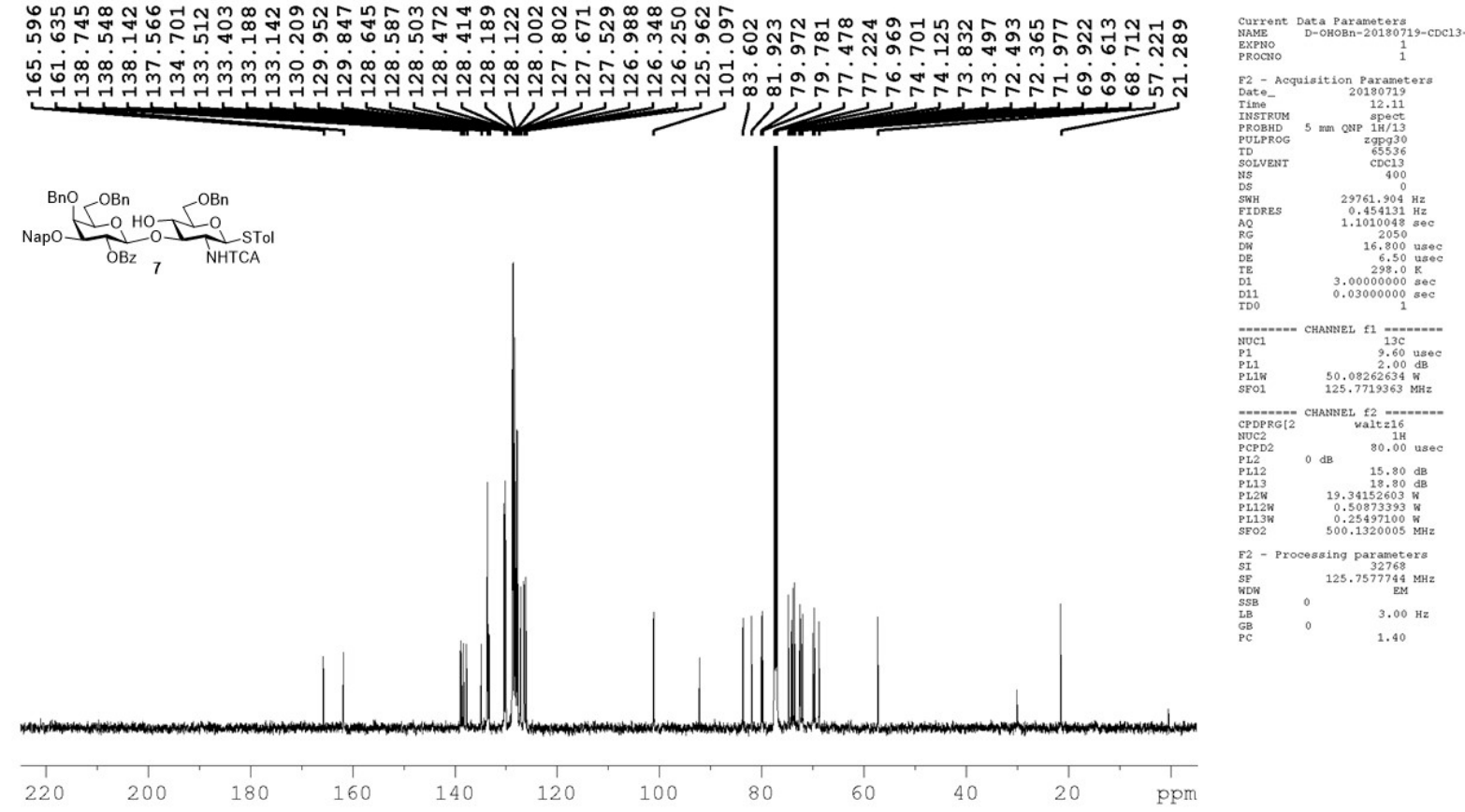

${ }^{13} \mathrm{C}$ NMR spectrum of compound $7\left(125 \mathrm{MHz}, \mathrm{CDCl}_{3}\right)$ 
${ }_{\mathrm{OBz}}^{8}{ }_{\mathrm{NHTCA}}^{\mathrm{OBn}}$
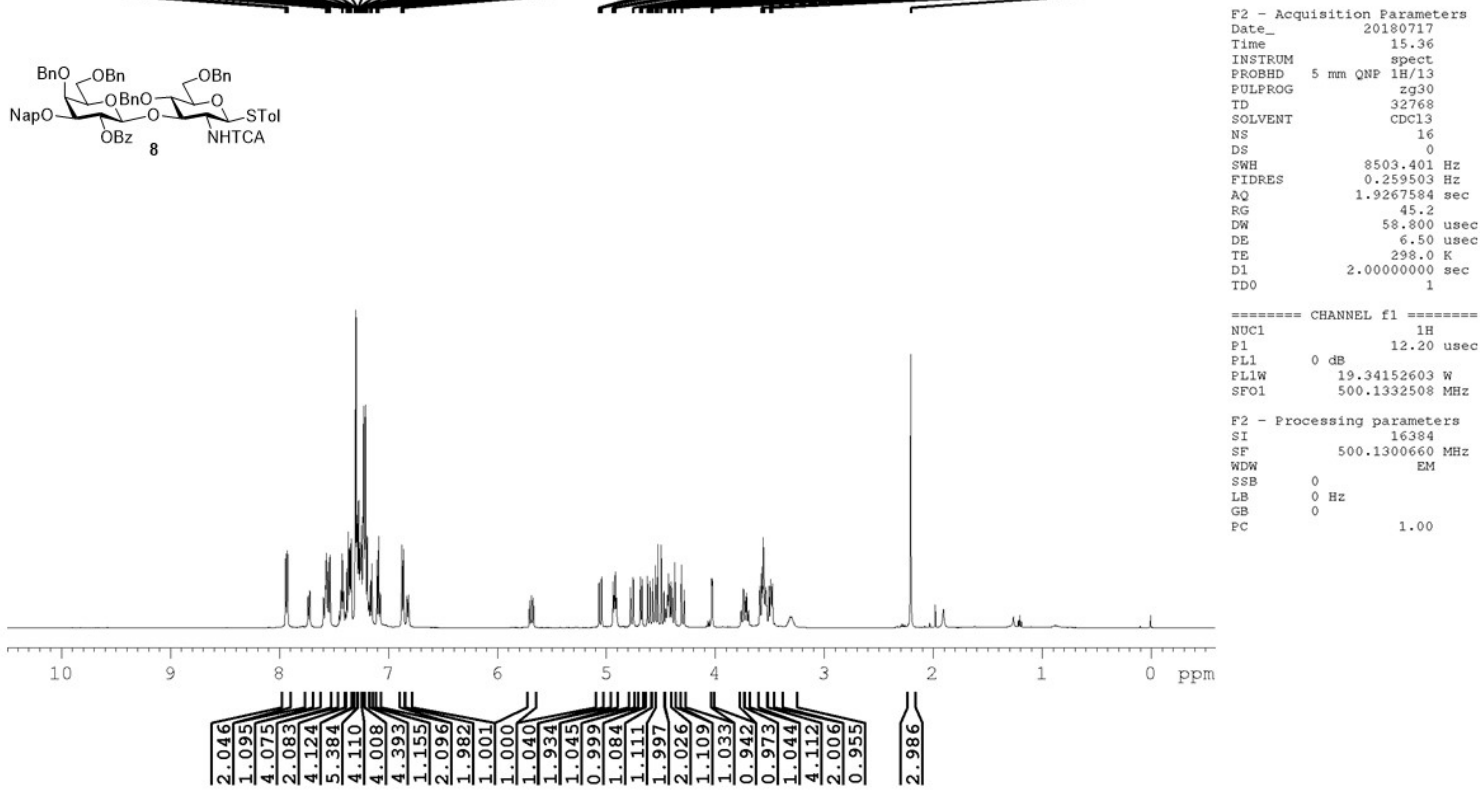

${ }^{1} \mathrm{H}$ NMR spectrum of compound $8\left(500 \mathrm{MHz}, \mathrm{CDCl}_{3}\right)$

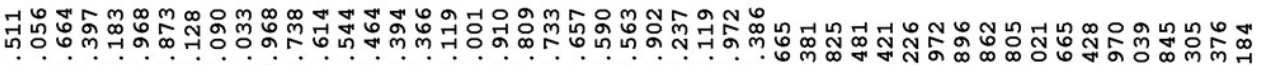
:

$\underbrace{{ }_{8}}_{\mathrm{OBz}} \underbrace{\mathrm{OBn}}_{\text {NHTCA }}$
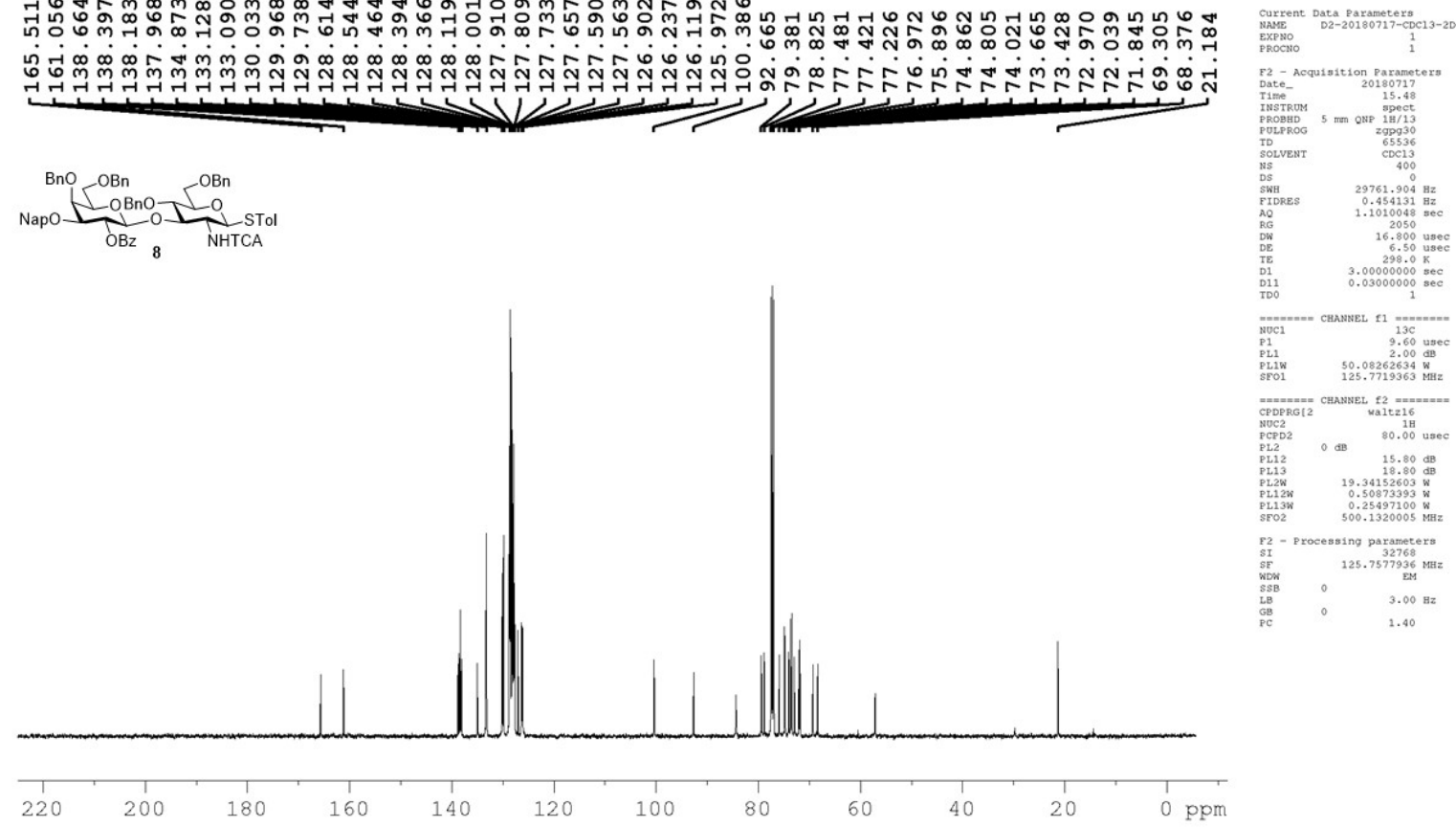

${ }^{13} \mathrm{C}$ NMR spectrum of compound $8\left(125 \mathrm{MHz}, \mathrm{CDCl}_{3}\right)$ 

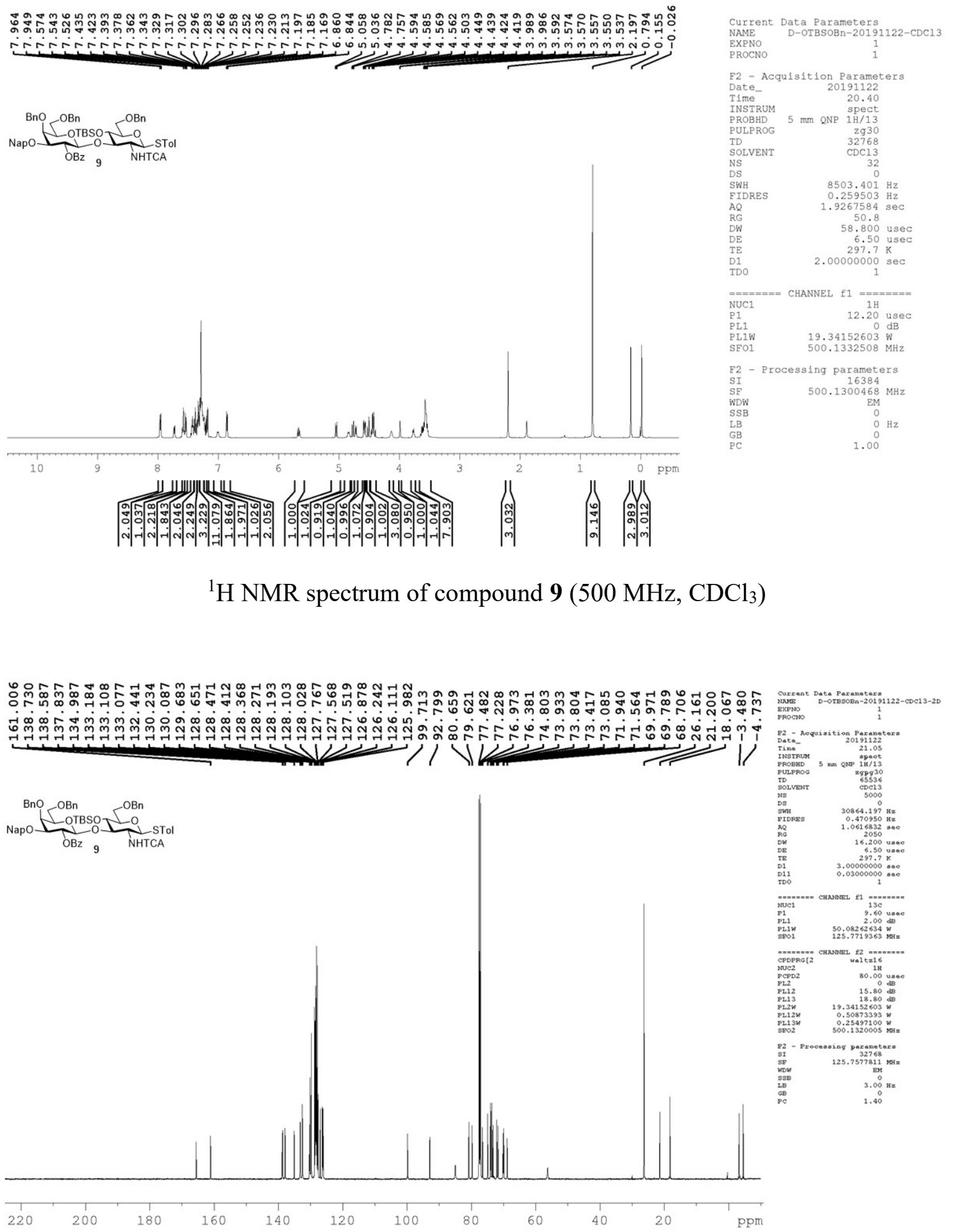

${ }^{13} \mathrm{C}$ NMR spectrum of compound $9\left(125 \mathrm{MHz}, \mathrm{CDCl}_{3}\right)$ 


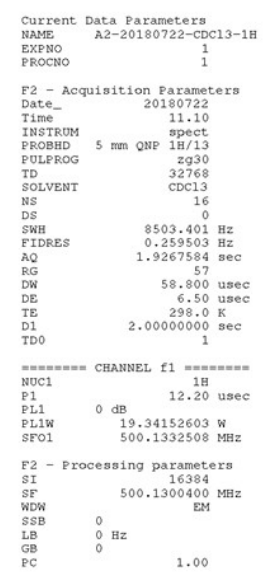

$$
\underbrace{10}_{\mathrm{OBz}}{ }_{\mathrm{NHTCA}}^{\mathrm{OBn}}
$$

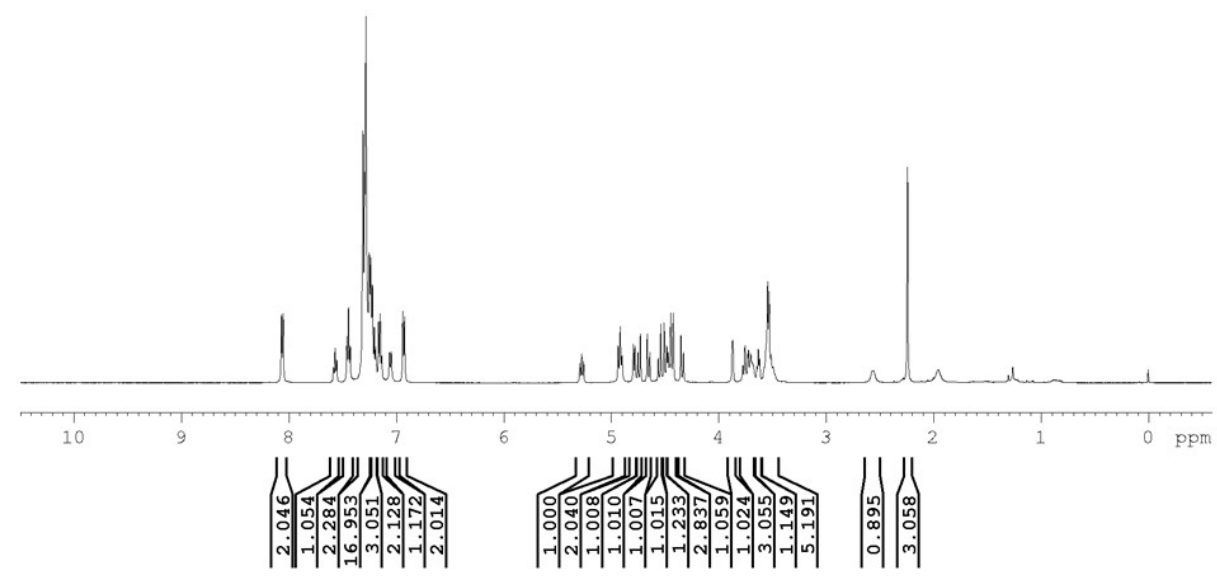

${ }^{1} \mathrm{H}$ NMR spectrum of compound $\mathbf{1 0}\left(500 \mathrm{MHz}, \mathrm{CDCl}_{3}\right)$

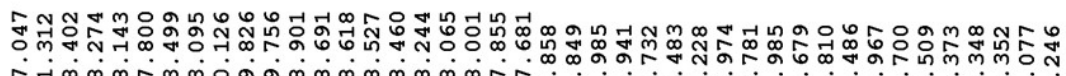

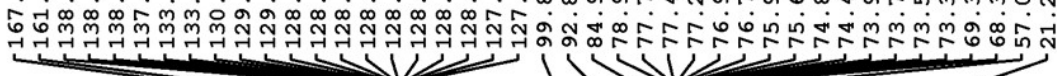

$\underbrace{10}_{\mathrm{OBz}} \underbrace{\mathrm{OBn}}_{\mathrm{NHTCA}}$
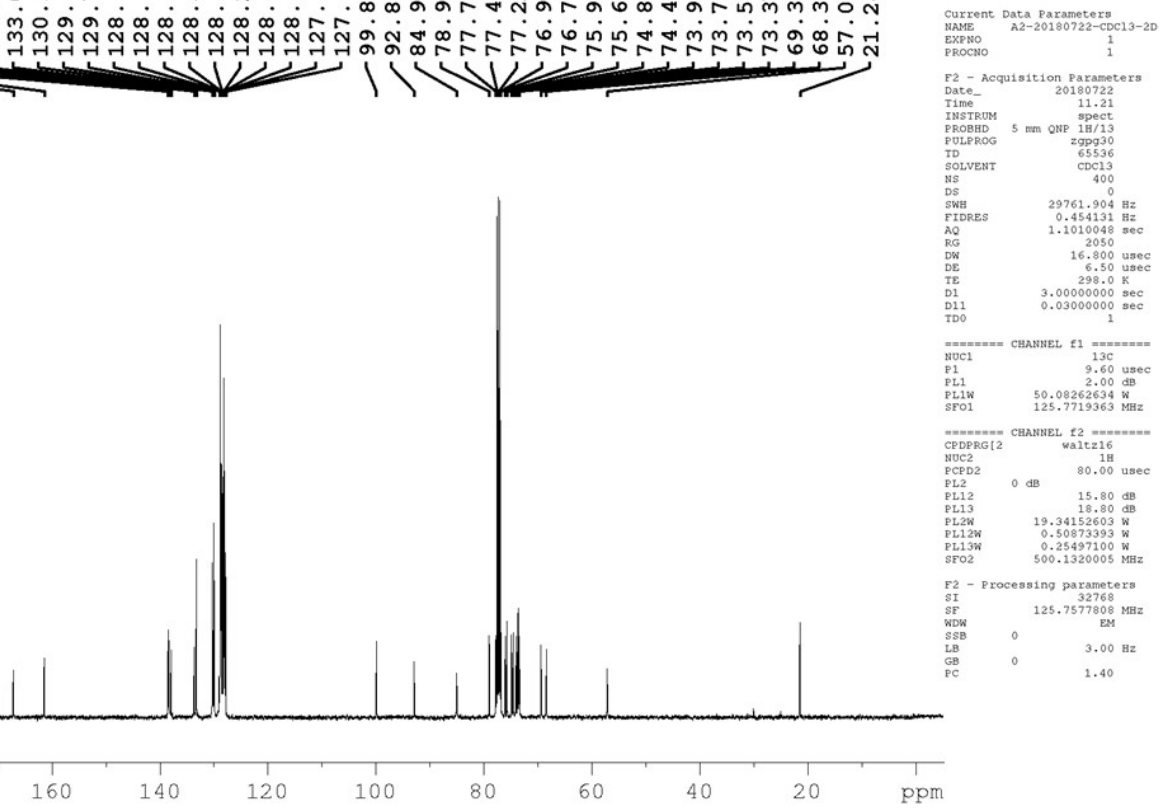

${ }^{13} \mathrm{C}$ NMR spectrum of compound $10\left(125 \mathrm{MHz}, \mathrm{CDCl}_{3}\right)$ 


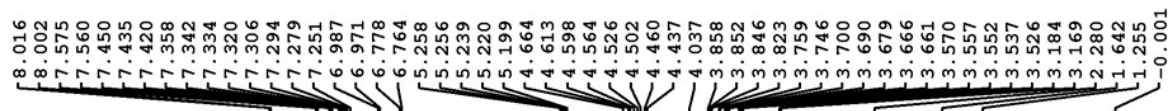

${ }_{\mathrm{OBz}}^{11}$

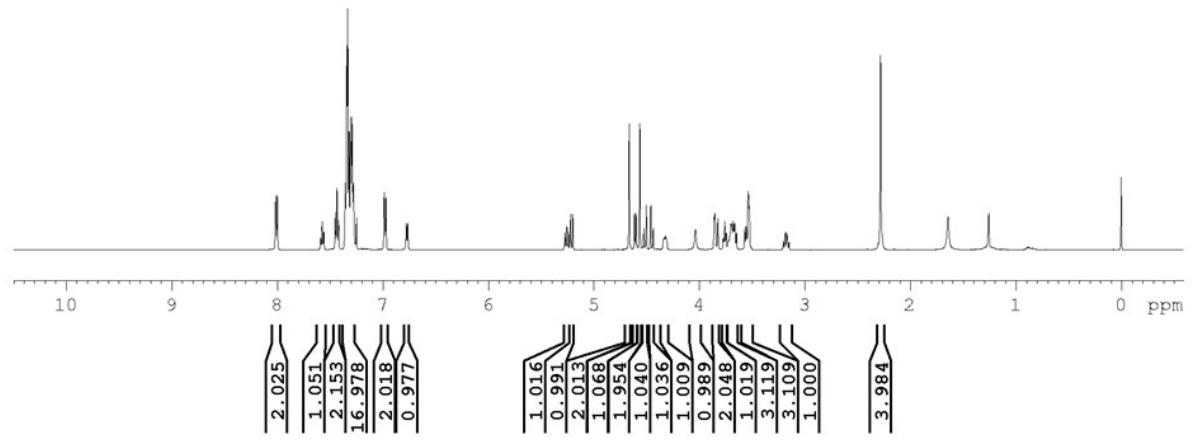

${ }^{1} \mathrm{H}$ NMR spectrum of compound $11\left(500 \mathrm{MHz}, \mathrm{CDCl}_{3}\right)$
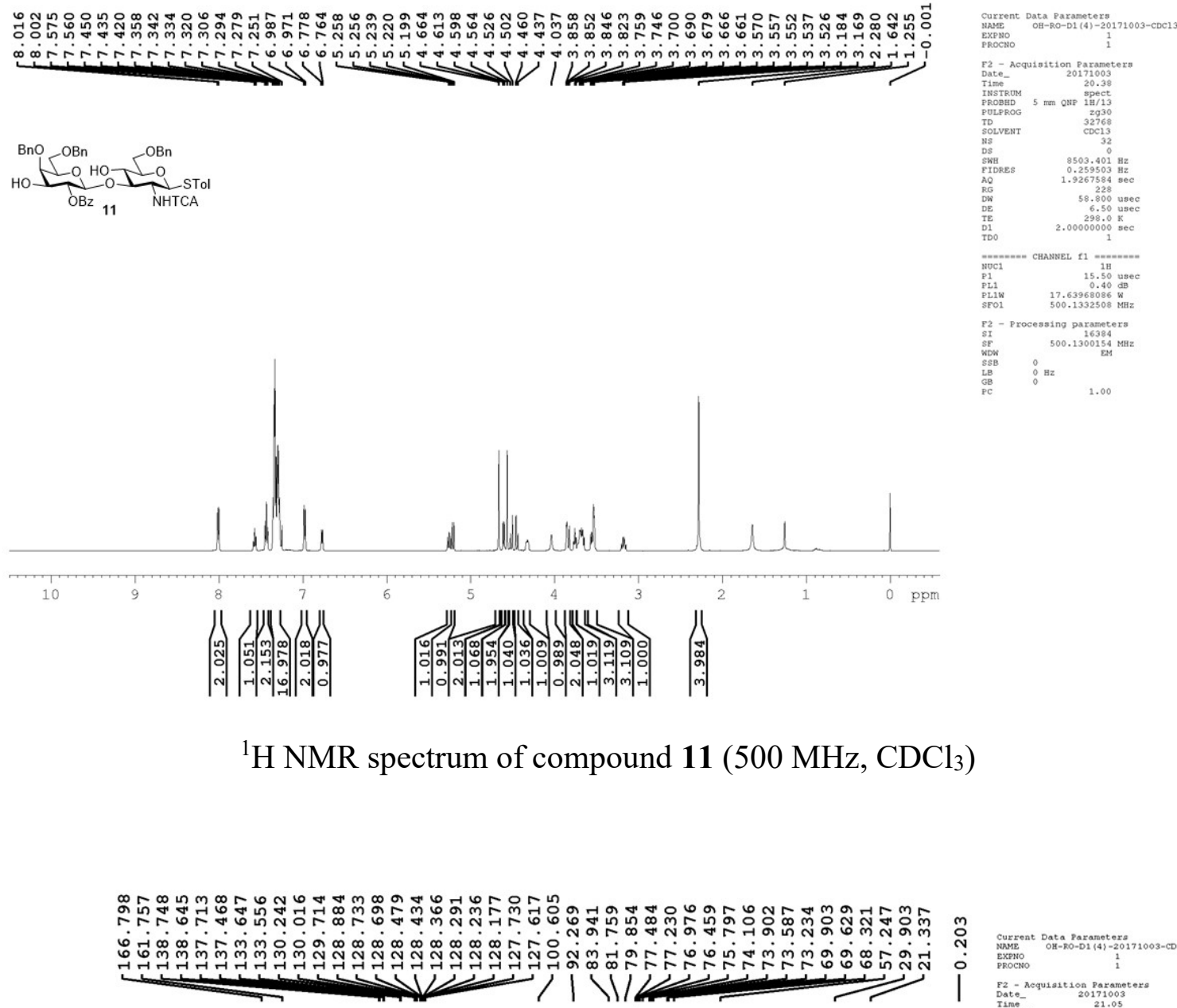

$\underbrace{11}_{\mathrm{OBz}} \underbrace{\mathrm{O}}_{\mathrm{NHTCA}}$ 作
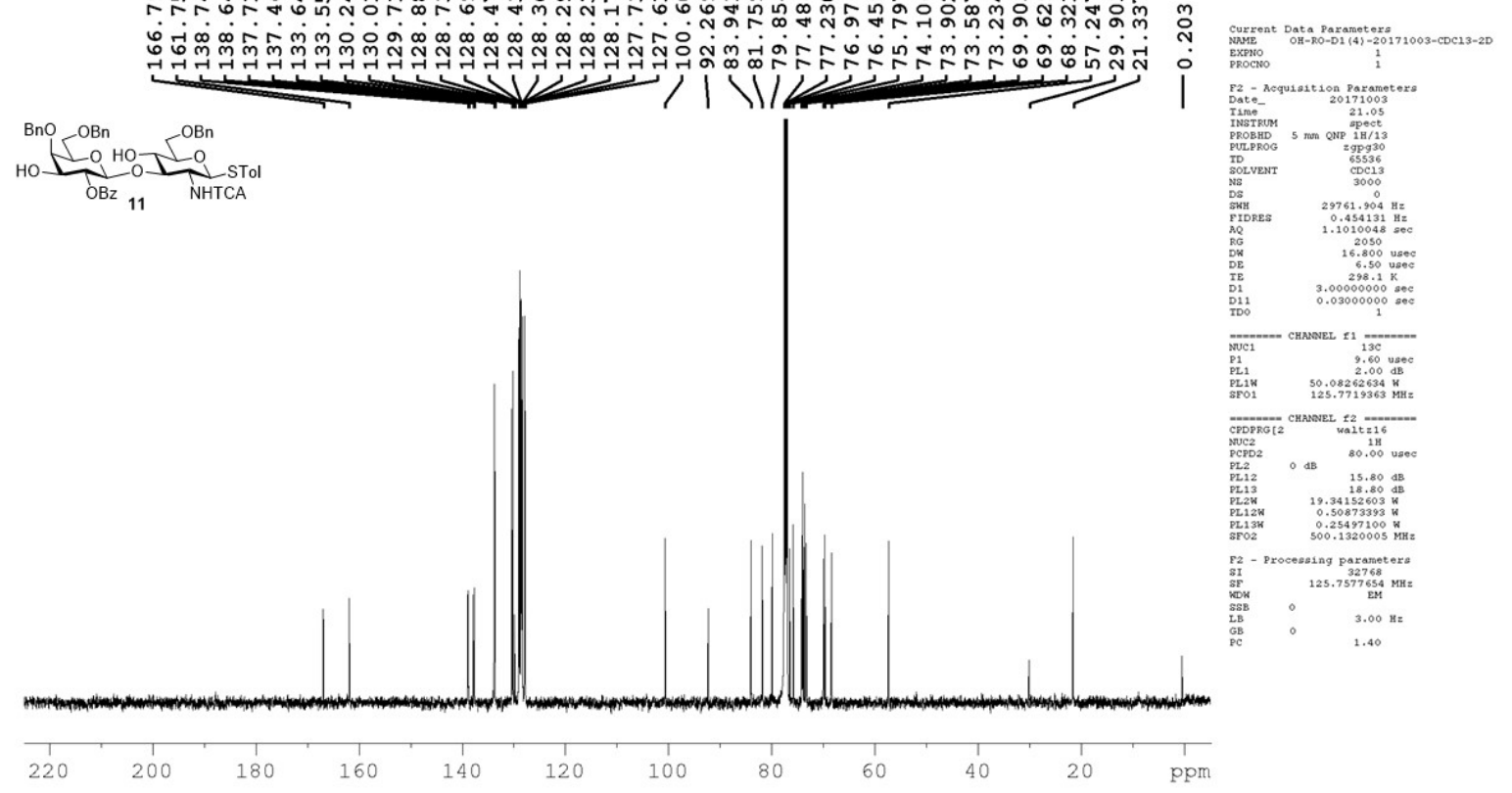

${ }^{13} \mathrm{C}$ NMR spectrum of compound $11\left(125 \mathrm{MHz}, \mathrm{CDCl}_{3}\right)$ 

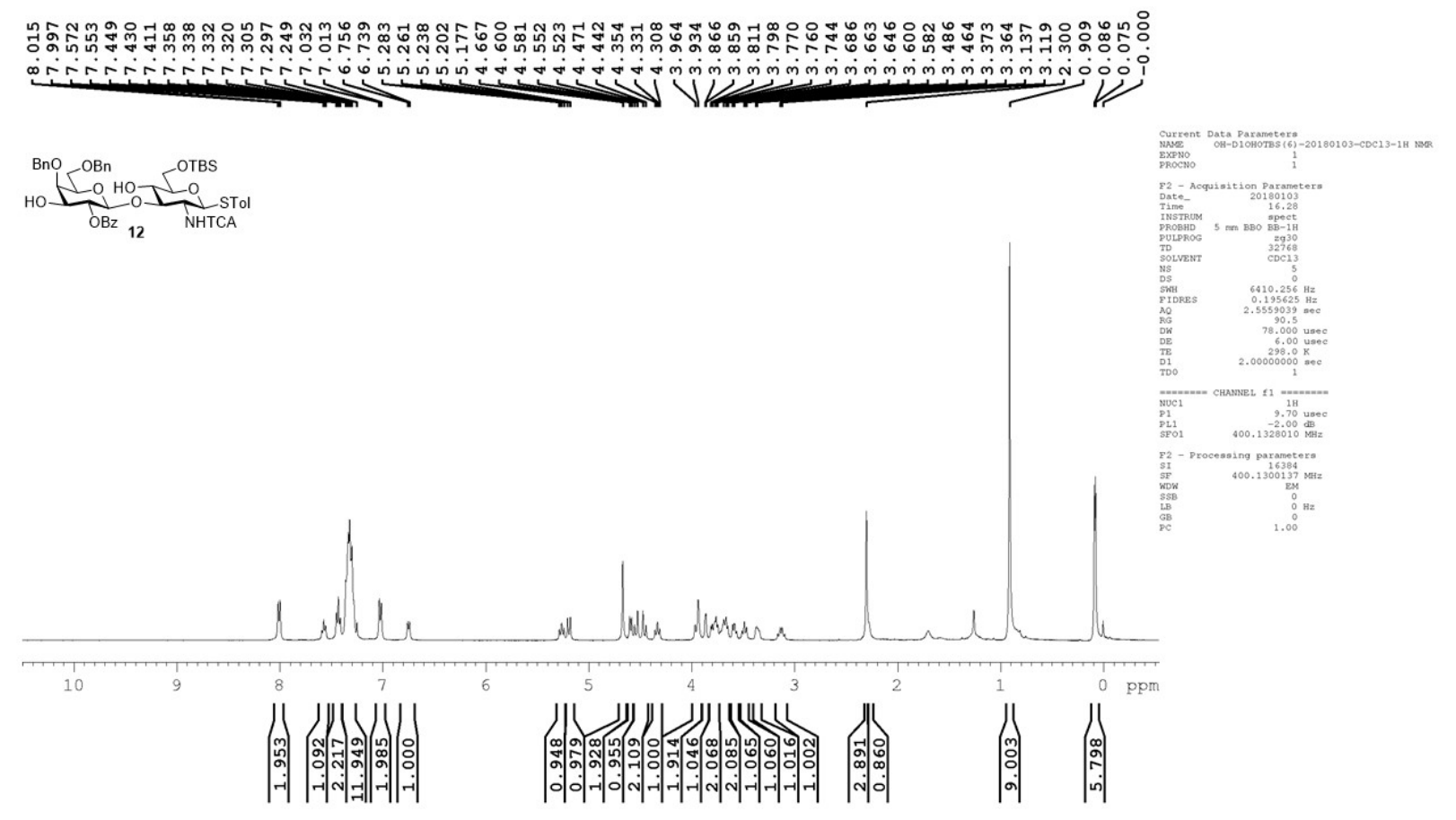

${ }^{1} \mathrm{H}$ NMR spectrum of compound $12\left(400 \mathrm{MHz}, \mathrm{CDCl}_{3}\right)$

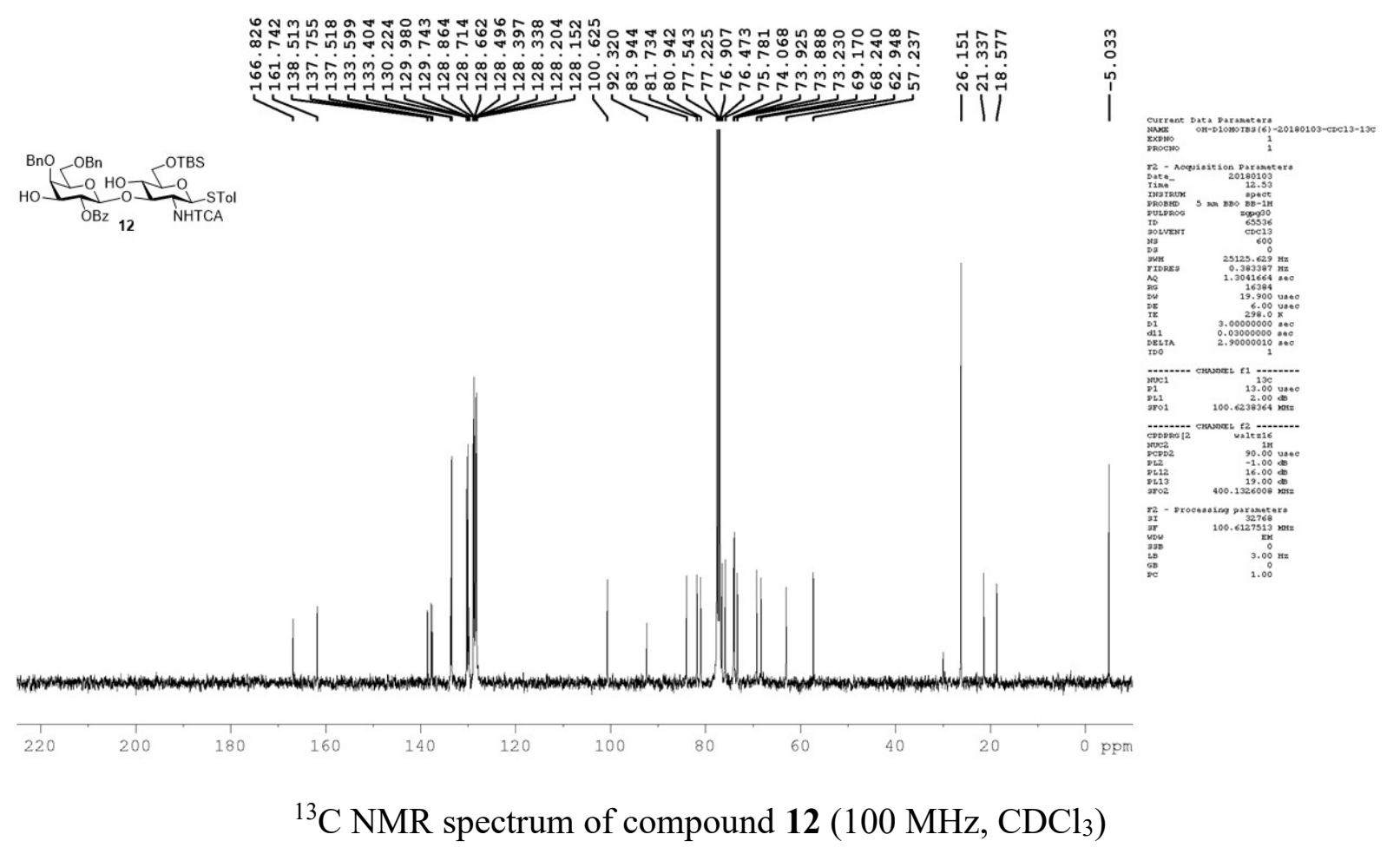


$\underbrace{13}_{\mathrm{OBz}}{ }_{\mathrm{NHTCA}}^{\mathrm{OBn}}$
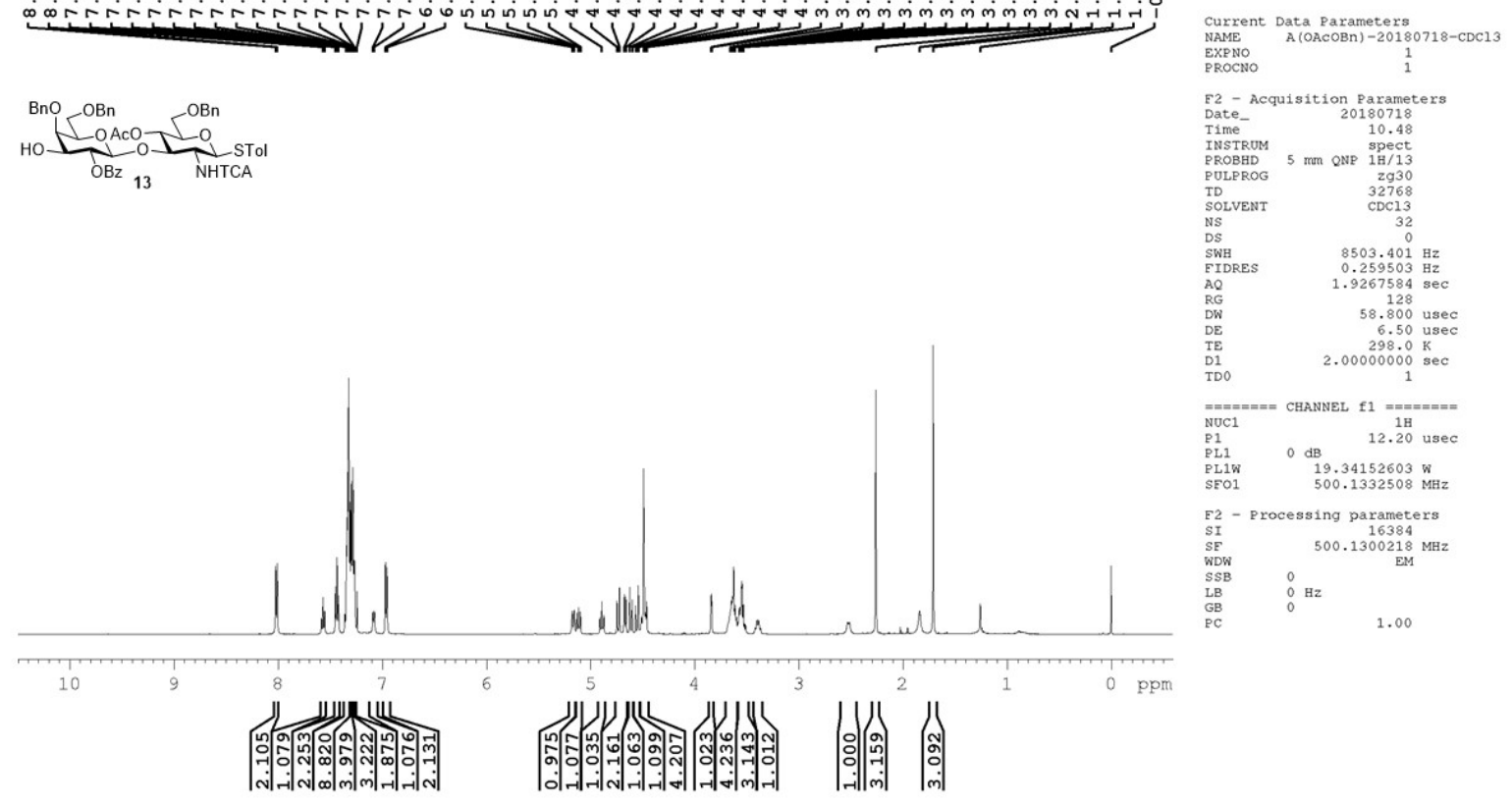

${ }^{1} \mathrm{H}$ NMR spectrum of compound $13\left(500 \mathrm{MHz}, \mathrm{CDCl}_{3}\right)$

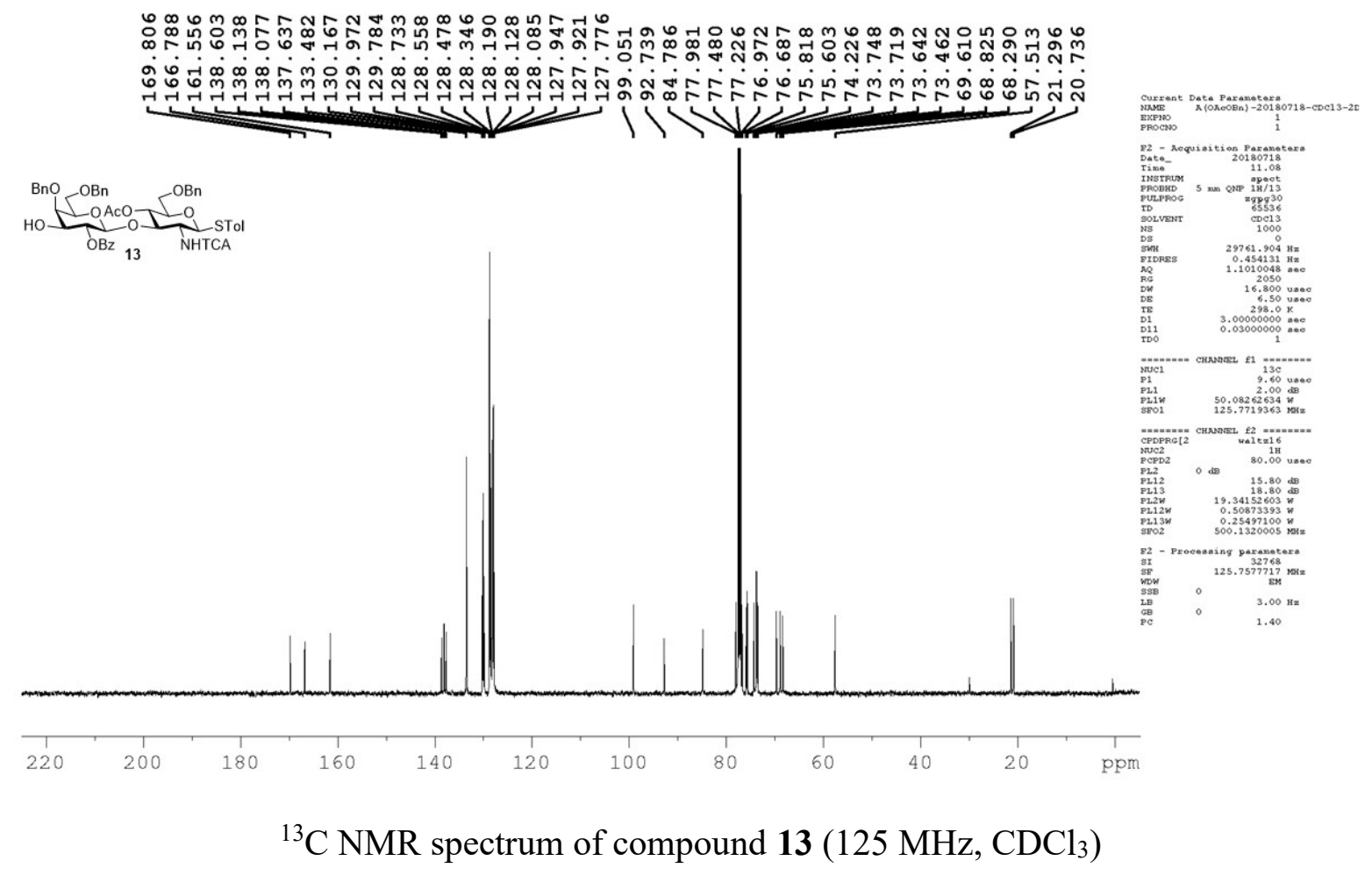



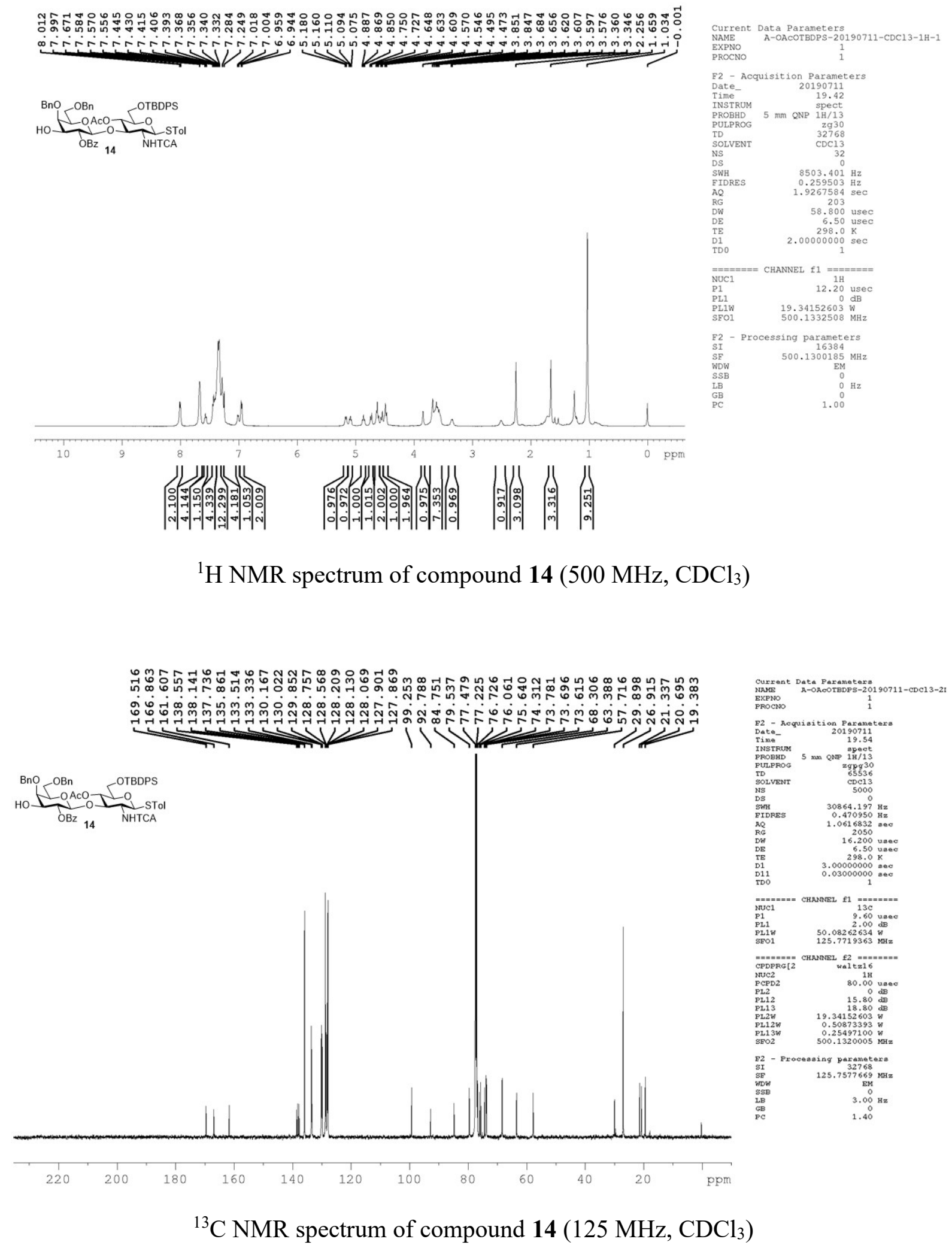

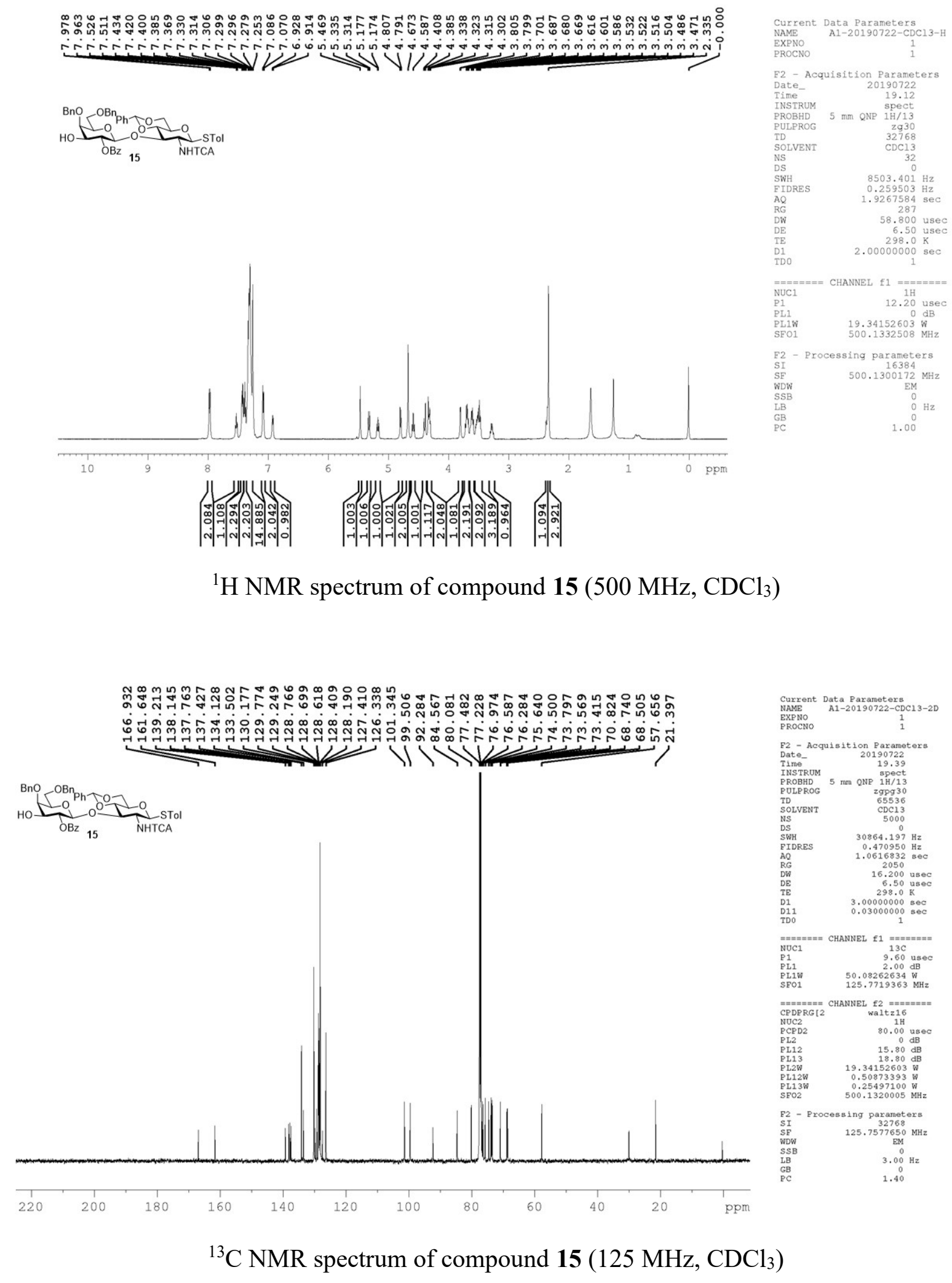
$\underbrace{\mathrm{O}}_{\mathrm{OBz}}{ }_{\mathrm{NHTCA}}^{\mathrm{OBn}}$

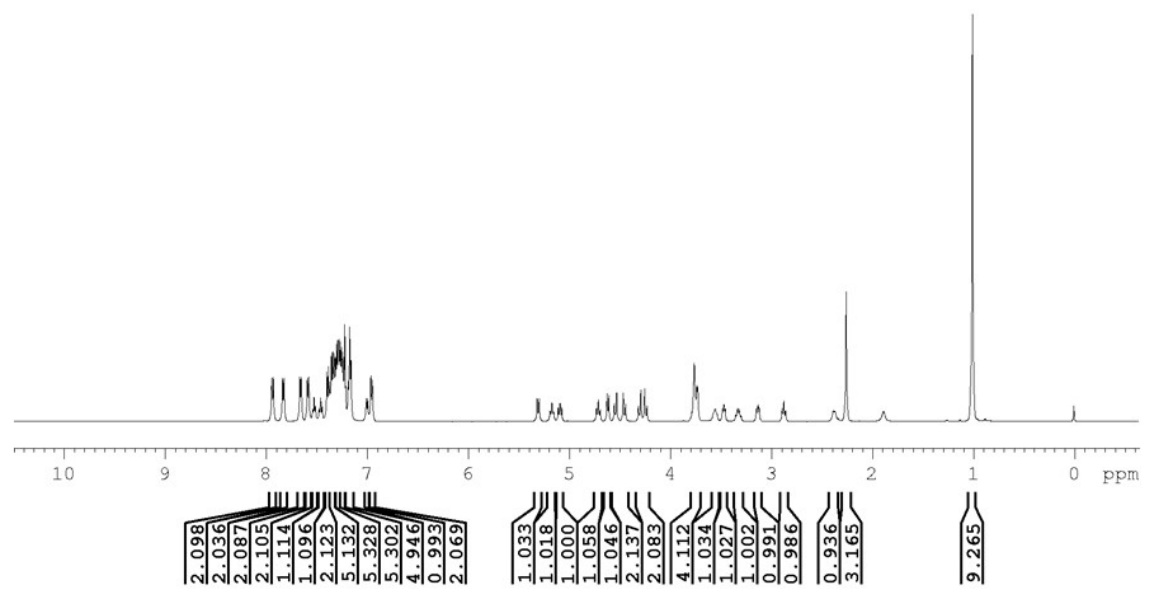

F2 - Acquisition Parameters

Date__ 20190716

Time $\quad 15.07$

INSTRUM spect

$\begin{array}{lll}\text { PROBHD } 5 \mathrm{~mm} \text { QNP } & 1 \mathrm{H} / 13 \\ \text { PULPROG } & \mathrm{zg} 30\end{array}$

SOLVENT

NS

SWH $8503.401 \mathrm{~Hz}$

FIDRES

$\begin{array}{lr}\text { AQ } & 1.9267584 \mathrm{sec} \\ \text { RG } & 64 \mathrm{us} \\ D W & 58.800 \mathrm{usec} \\ D E & 6.50 \mathrm{usec}\end{array}$

6.50 usec

$2.00000000 \mathrm{sec}$

$\begin{array}{lr}=======\text { CHANNEL } \mathrm{f} 1 \mathrm{l}======= \\ \text { NUC1 } \\ 1 \mathrm{H} \\ \text { P1 } & 12.20 \mathrm{usec} \\ \text { PL1 } & 0 \mathrm{~dB} \\ \text { PL1W } & 19.34152603 \mathrm{~W} \\ \text { SF01 } & 500.1332508 \mathrm{MHz}\end{array}$

F2 - Processing parameters

SI 16384

SF $\quad 500.1300343$

SSB

LB
GB
PC

${ }_{0}^{0} \mathrm{~Hz}$
0
1.00

${ }^{1} \mathrm{H}$ NMR spectrum of compound $16\left(500 \mathrm{MHz}, \mathrm{CDCl}_{3}\right)$

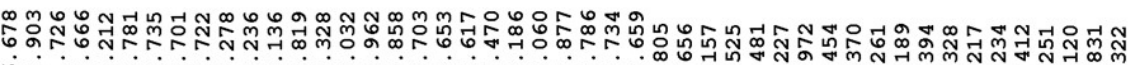
: 1.

$\underbrace{16}_{\mathrm{OBz}} \underbrace{\mathrm{O}}_{\text {NHTCA }}$
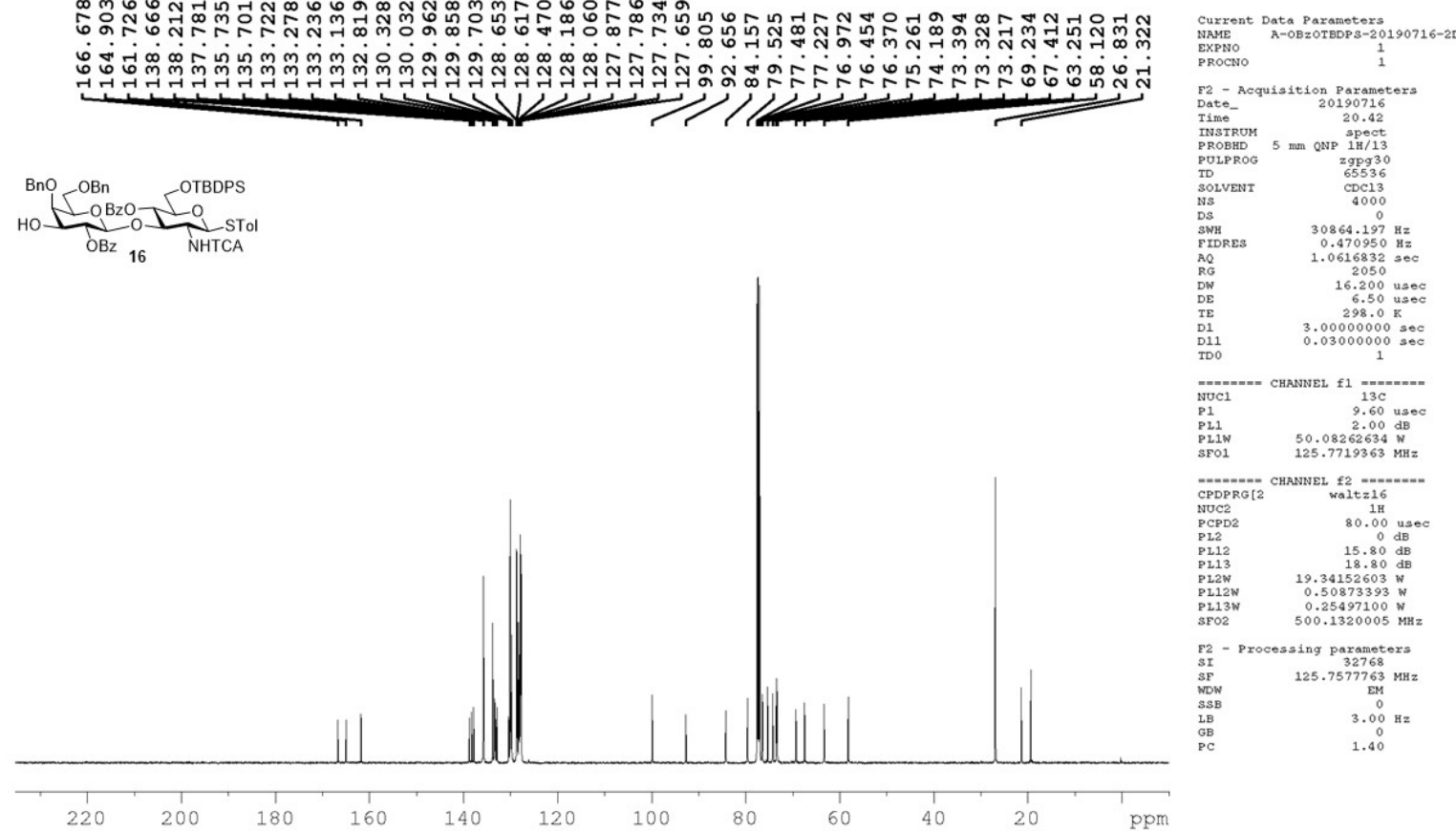

${ }^{13} \mathrm{C}$ NMR spectrum of compound $\mathbf{1 6}\left(125 \mathrm{MHz}, \mathrm{CDCl}_{3}\right)$ 


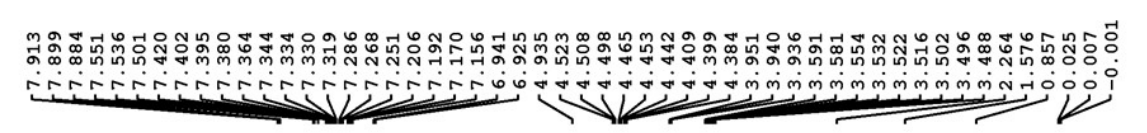

$\underbrace{\mathrm{O}}_{\mathrm{OBz}}$

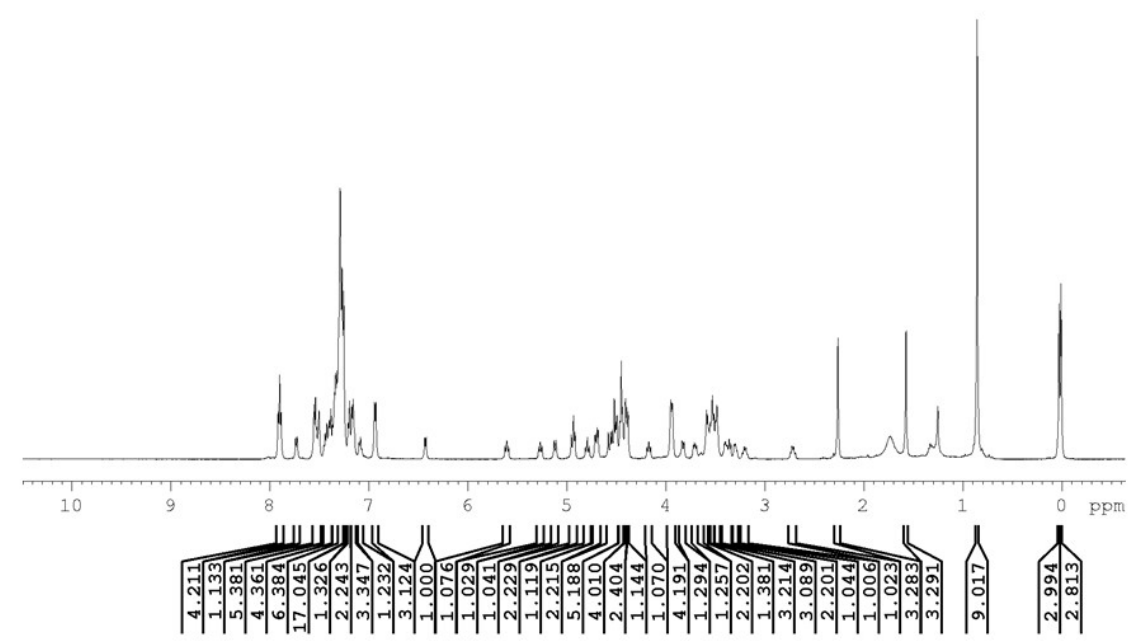

${ }^{1} \mathrm{H}$ NMR spectrum of compound $17\left(500 \mathrm{MHz}, \mathrm{CDCl}_{3}\right)$

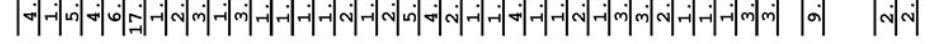

Current Data Parameters
NAME D-OHOTBS-A-OAcOBn-20190913-CDC13
EXPNO

PROCNO

F2 - Acquisition Parameters
Date- 20190913

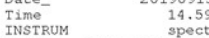

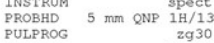

$\begin{array}{lr}\text { TD } & 32768 \\ \text { SOLVENT } & \text { CDC13 } \\ \text { NS } & 32 \\ \text { DS } & 32\end{array}$

$\begin{array}{lc}\text { NS } & 32 \\ \text { DS } & 0 \\ \text { SWH } & 8503.401 \mathrm{~Hz} \\ \text { FIDRES } & 0.259503 \mathrm{~Hz} \\ \text { A } & 1.9267584\end{array}$

$\begin{array}{lc}\text { MQDRES } & 0.225784 \mathrm{~Hz} \\ \text { AQ } & 1.926758 \mathrm{sec} \\ \text { RG } & 228\end{array}$

$\begin{array}{rr}6 \mathrm{TE} & 6.50 \mathrm{usec} \\ \mathrm{TE} & 298.0 \mathrm{~K} \\ \mathrm{TE} & 2.00000000 \mathrm{sec}\end{array}$

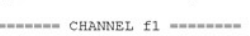

$\begin{array}{ll}\text { NUC1 } & 1 \mathrm{H} \\ \mathrm{P1} & 12.20 \mathrm{usec} \\ \mathrm{PL1} & 0\end{array}$

PL1W $\quad 19.34152603 \mathrm{w}$

F2 - Processing parameters

$\begin{array}{lc}\text { SI } & \text { Processing parameters } \\ \text { SF } & 16384 \\ S \text { S } & 500.1300180 \mathrm{MH}\end{array}$

$\begin{array}{lc}\text { WDW } & \text { EM } \\ \text { SSB } & 0 \\ \text { LB } & 0 \mathrm{~Hz} \\ \text { GB } & 0 \\ \text { PC } & 1.00\end{array}$

1.00

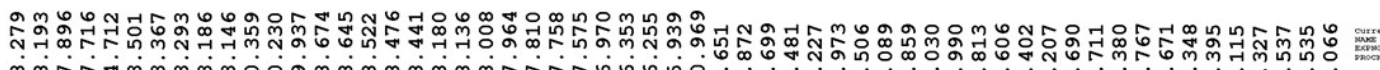

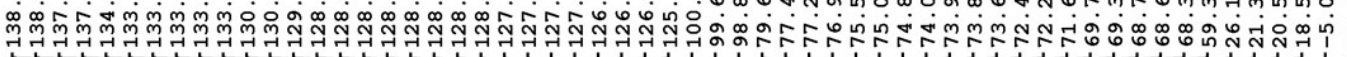
管 $\underbrace{\mathrm{OBn}}_{\mathrm{OBz}}$

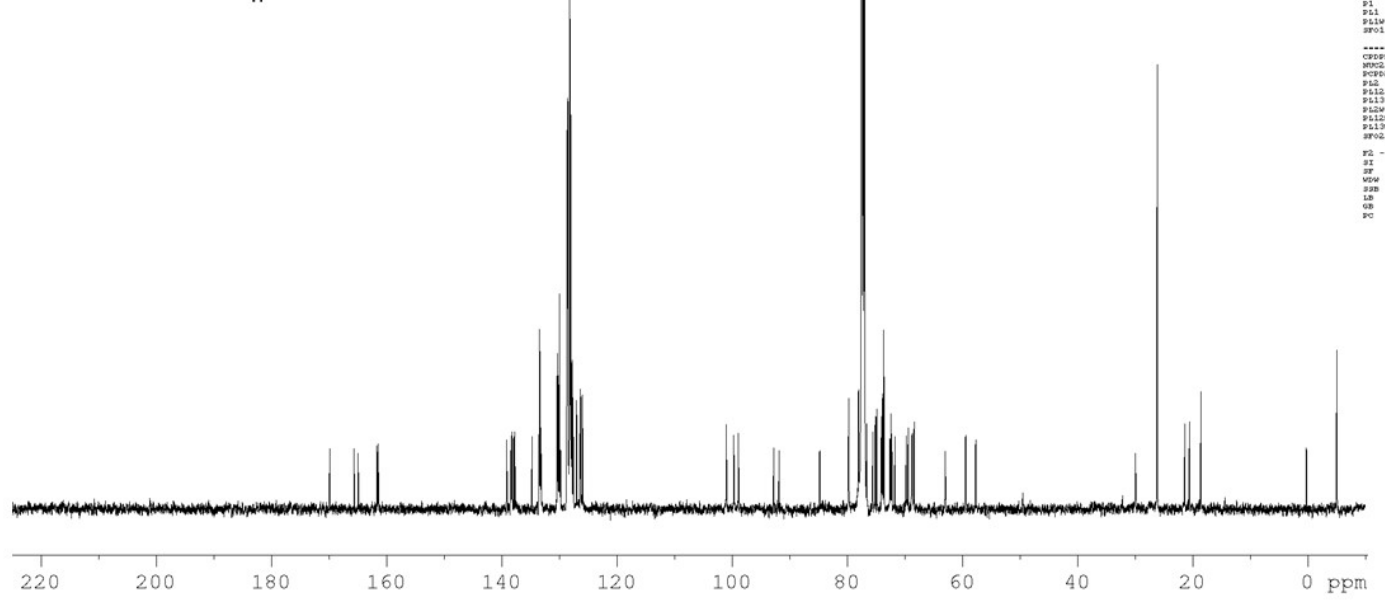

${ }^{13} \mathrm{C}$ NMR spectrum of compound $17\left(125 \mathrm{MHz}, \mathrm{CDCl}_{3}\right)$ 

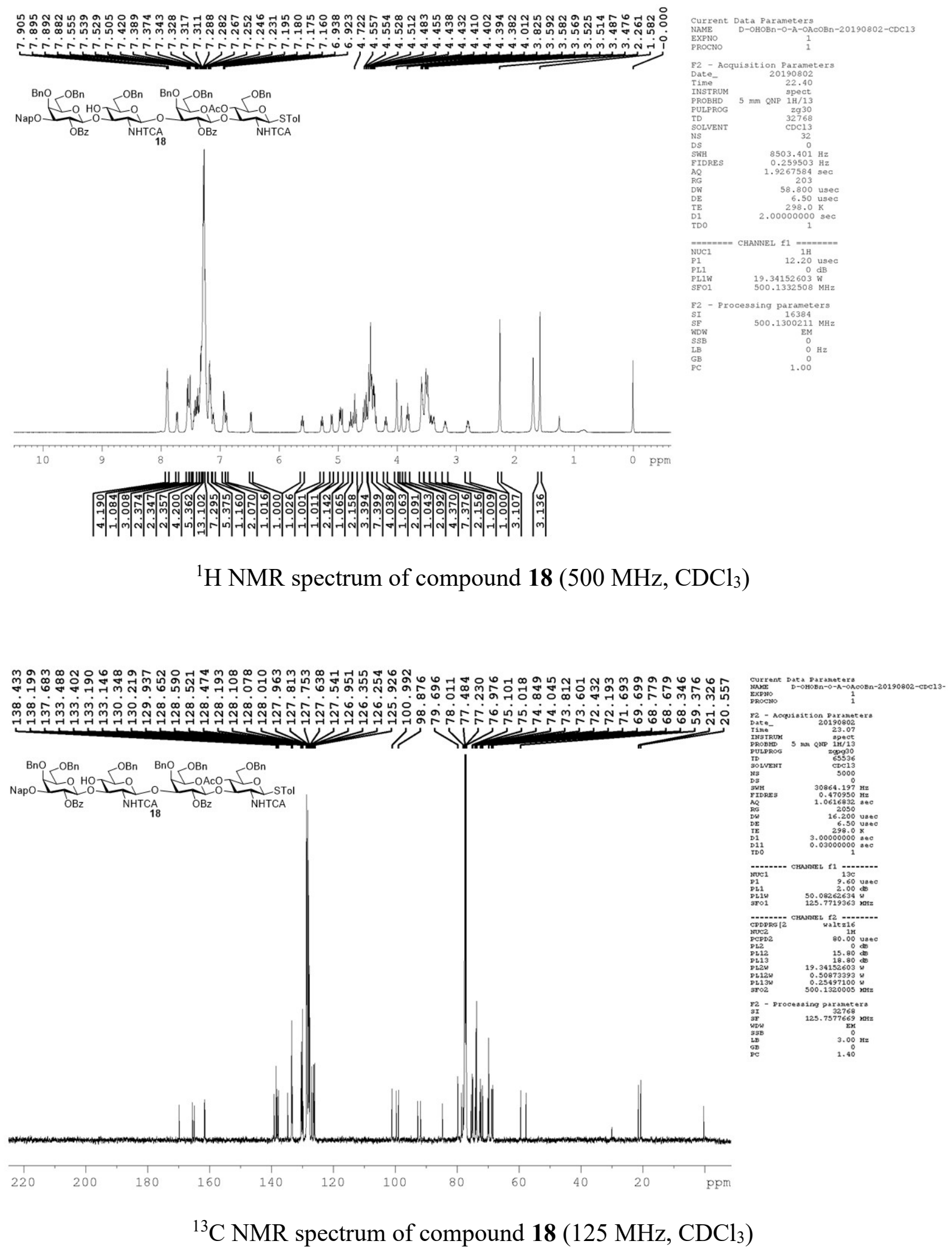


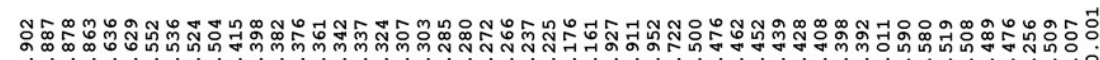

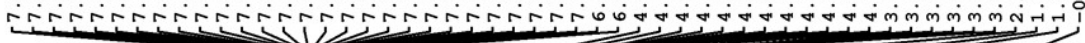

NapO $\underbrace{\mathrm{O}}_{\mathrm{OBz}} \underbrace{\mathrm{OBn}}_{\mathrm{NHTCA}}$

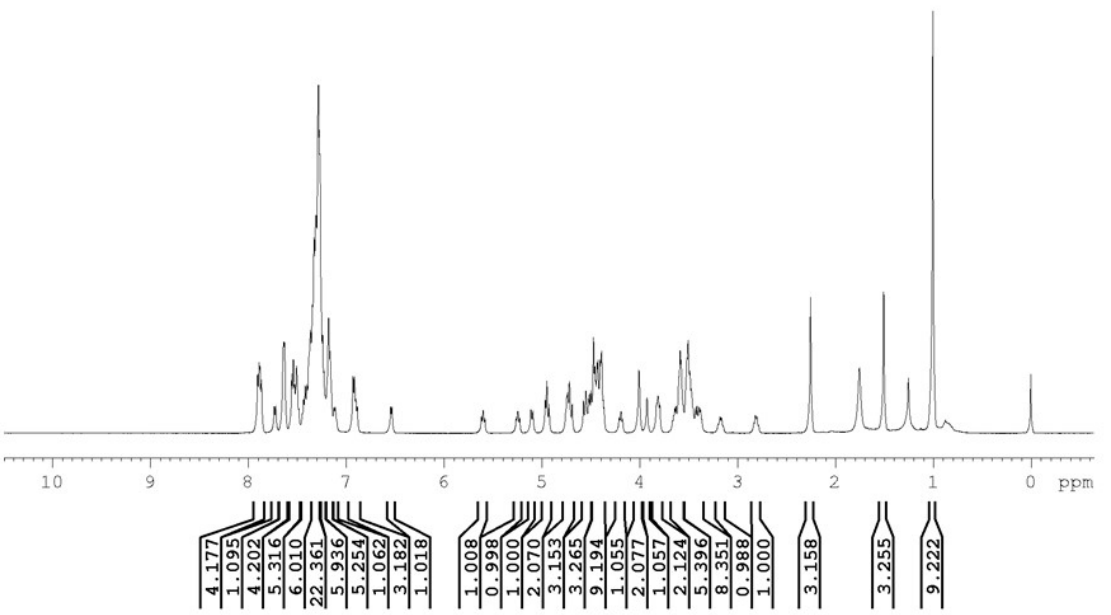

${ }^{1} \mathrm{H}$ NMR spectrum of compound $19\left(500 \mathrm{MHz}, \mathrm{CDCl}_{3}\right)$

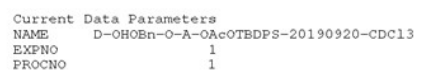

F2- Acquisition Parameters
$\begin{aligned} & \text { Date- } \\ & \text { Time }\end{aligned} 20190920$
21.14

Time
INSTRUM

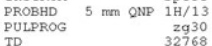

SOLVENT

$\begin{array}{lc}\text { DS } & 32 \\ \text { SWH } & 8503.401 \mathrm{~Hz} \\ \text { FIDRES } & 0.259503 \mathrm{~Hz}\end{array}$

\begin{tabular}{lr} 
FIDRES & $0.259503 \mathrm{~Hz}$ \\
AQ & $1.9267584 \mathrm{sec}$ \\
RG & 90.5 \\
\hline & 58.800 use
\end{tabular}

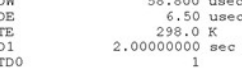

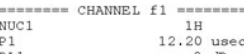

$\begin{array}{lr} & 0 \\ \text { PL1 } & \text { dB } \\ \text { PL1W } & 19.34152603 \mathrm{k} \\ \text { SFO1 } & 500.1332508 \mathrm{MHz}\end{array}$

${ }_{S 1}^{F 2}$ - Processing parameters

$\begin{array}{cc}\text { SE } & 500.1300247 \mathrm{MHz} \\ \text { WDW } & \text { EM } \\ \text { SSB } & 0 \\ \text { LB } & 0 \mathrm{~Hz} \\ \text { GB } & 0 \\ \text { PC } & 1.00\end{array}$

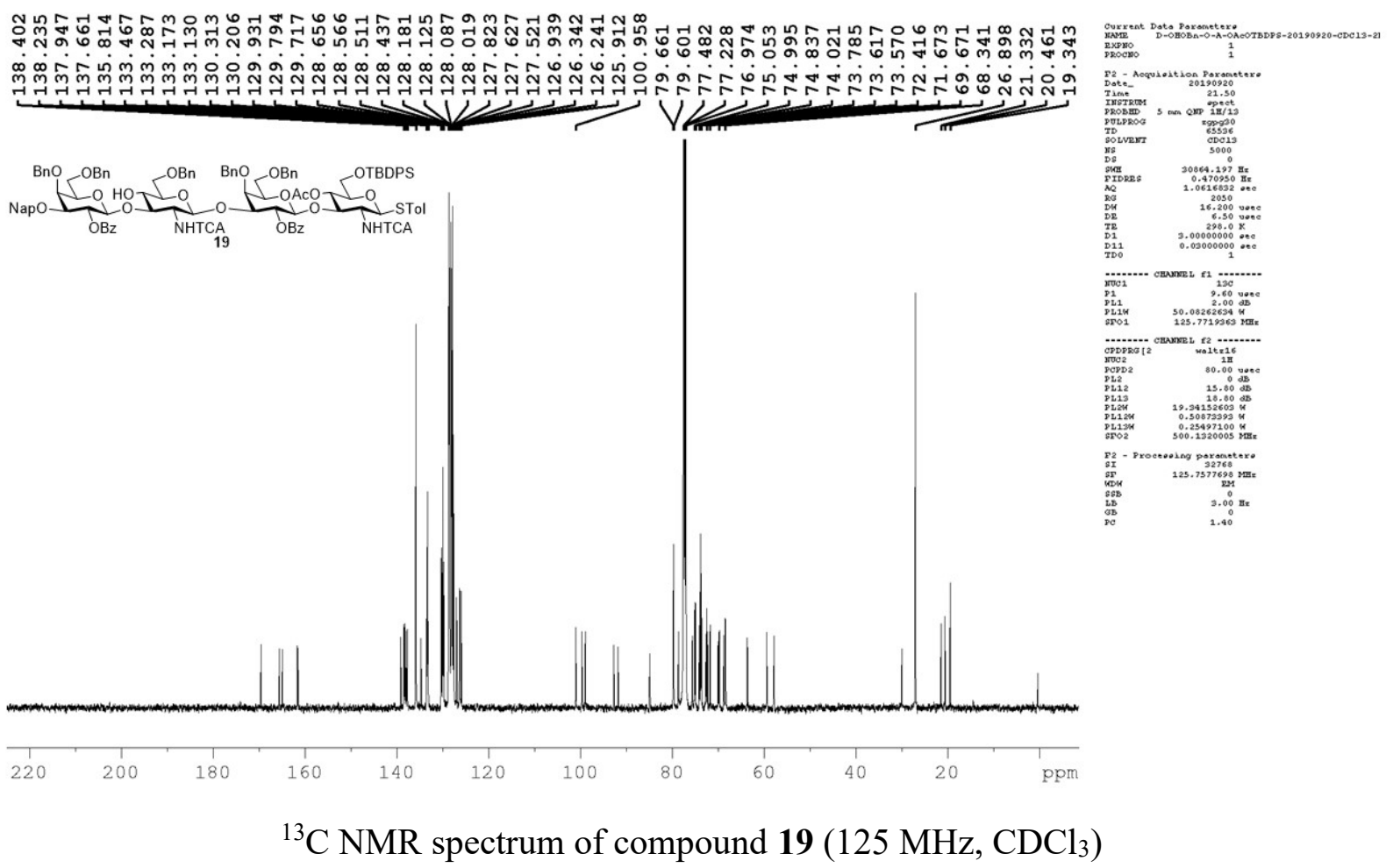



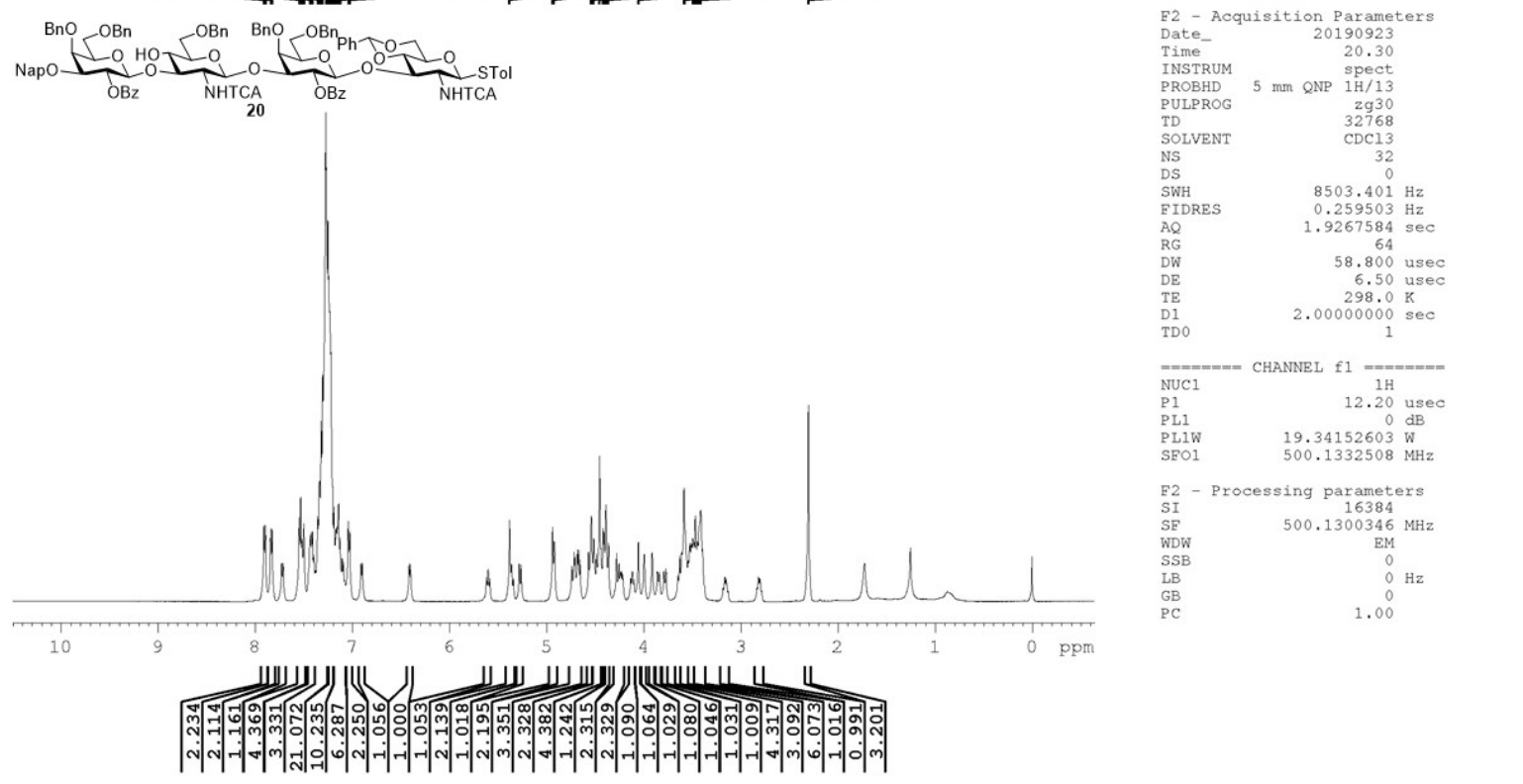

${ }^{1} \mathrm{H}$ NMR spectrum of compound $20\left(500 \mathrm{MHz}, \mathrm{CDCl}_{3}\right)$

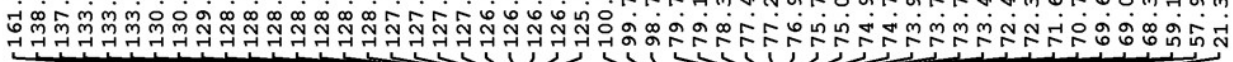

${ }_{\mathrm{OBz}}^{\mathrm{O}_{\mathrm{NHTCA}}^{\mathrm{OBn}}} \underbrace{\mathrm{OBn}}_{\mathrm{OBz}}$
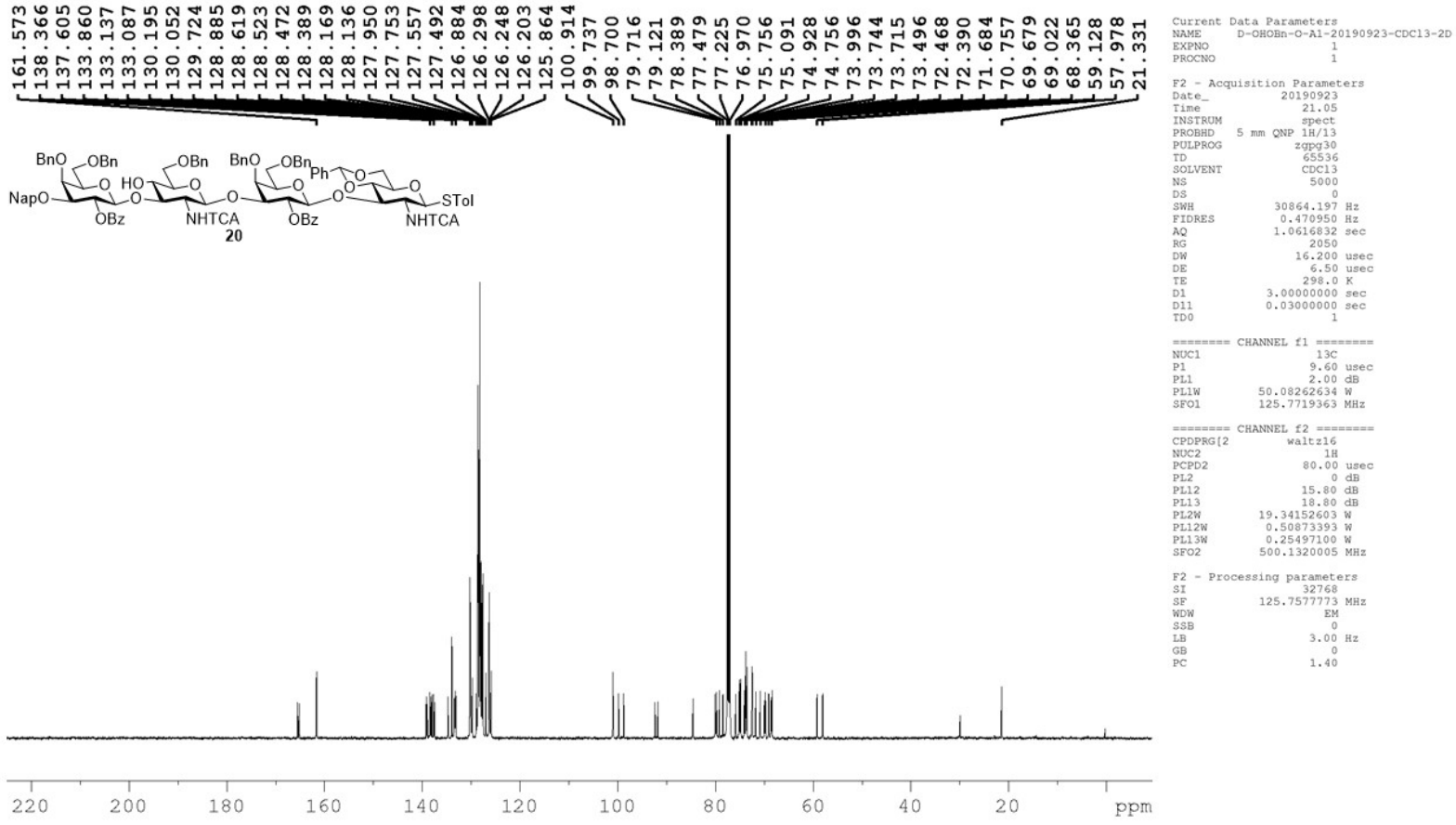

${ }^{13} \mathrm{C}$ NMR spectrum of compound $20\left(125 \mathrm{MHz}, \mathrm{CDCl}_{3}\right)$ 


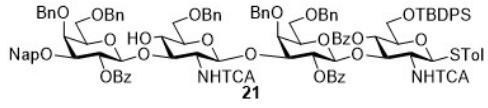

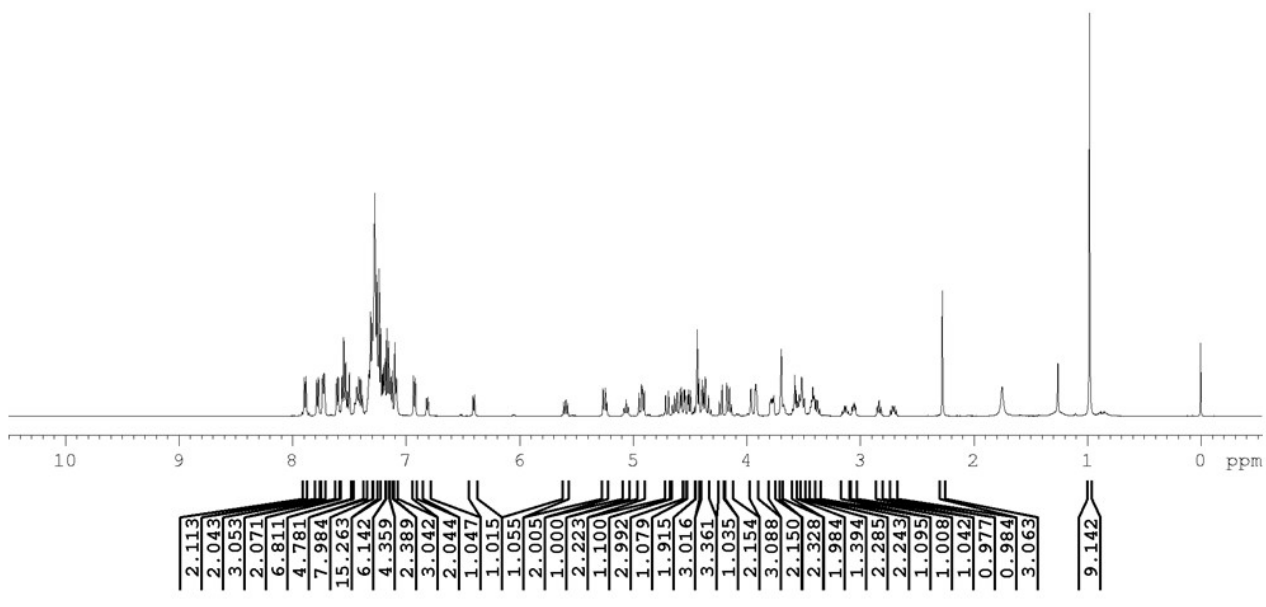

${ }^{1} \mathrm{H}$ NMR spectrum of compound $21\left(500 \mathrm{MHz}, \mathrm{CDCl}_{3}\right)$

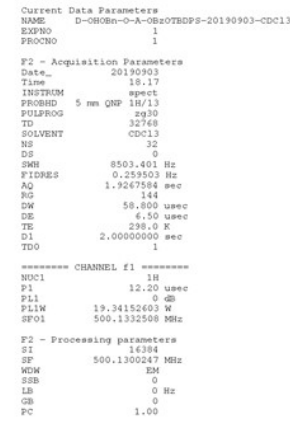

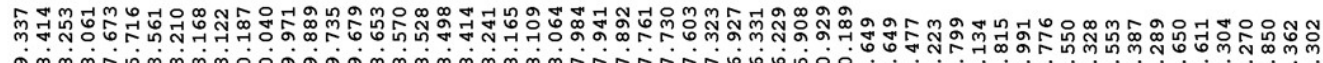

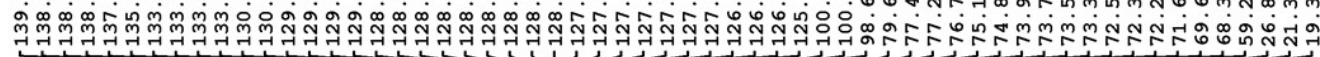
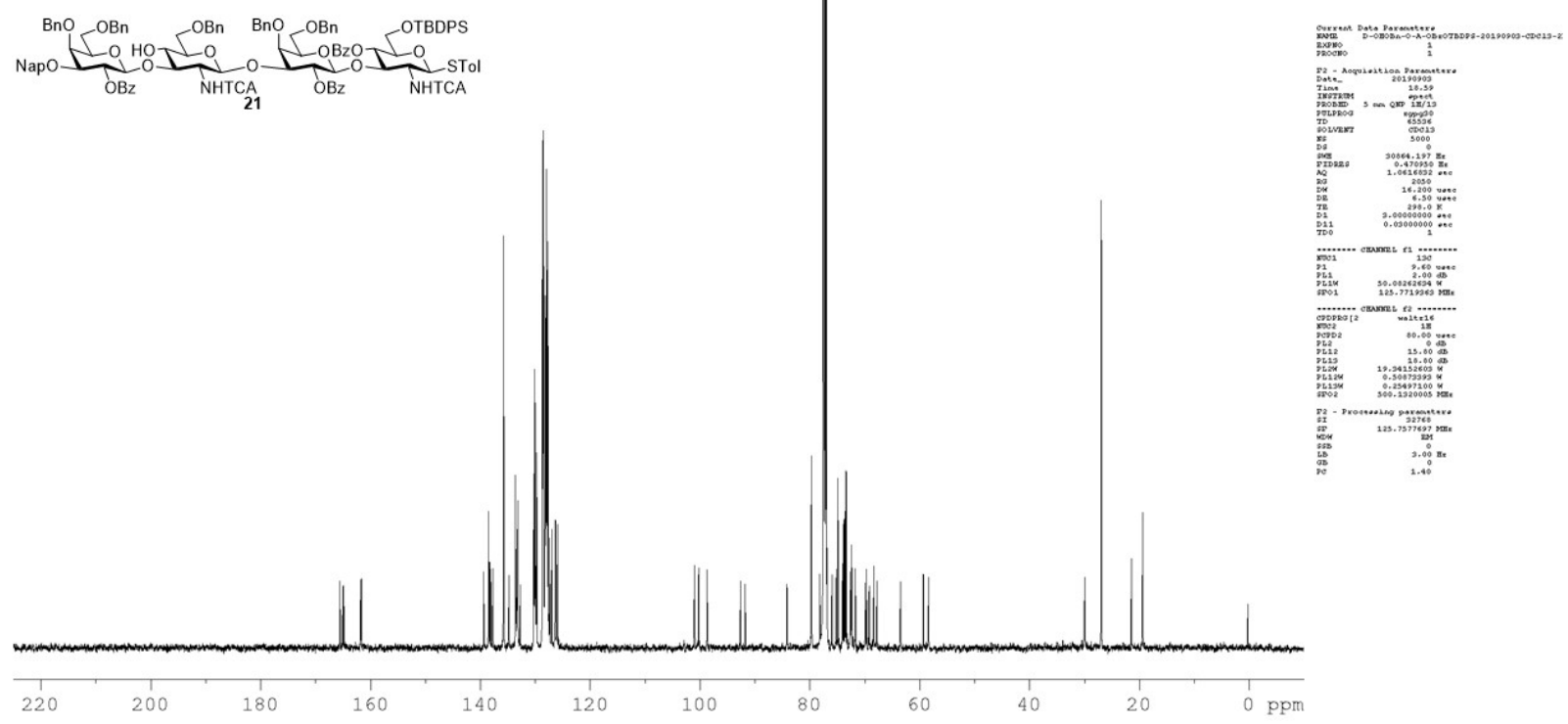

${ }^{13} \mathrm{C}$ NMR spectrum of compound $21\left(125 \mathrm{MHz}, \mathrm{CDCl}_{3}\right)$ 


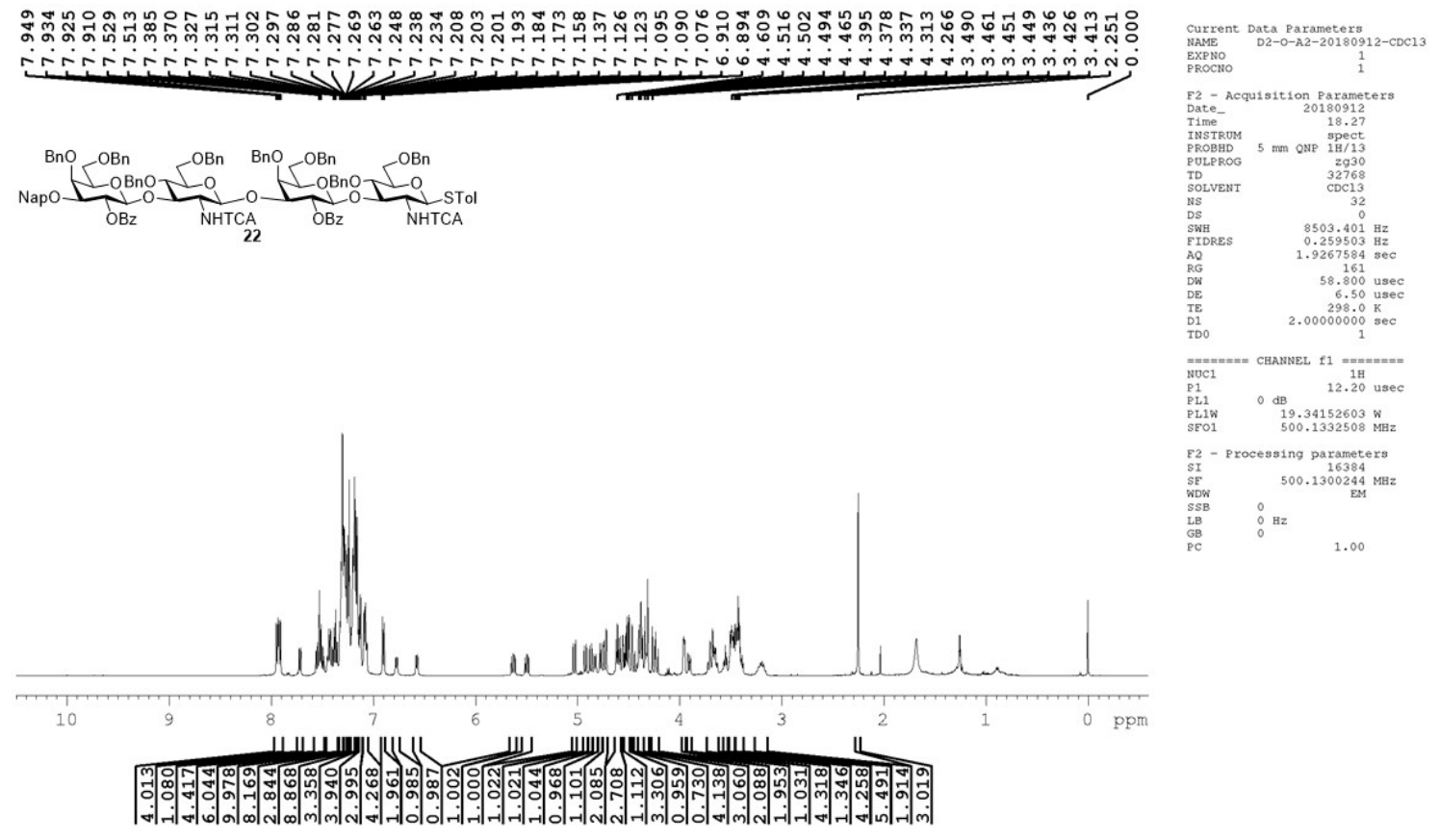

${ }^{1} \mathrm{H}$ NMR spectrum of compound $22\left(500 \mathrm{MHz}, \mathrm{CDCl}_{3}\right)$

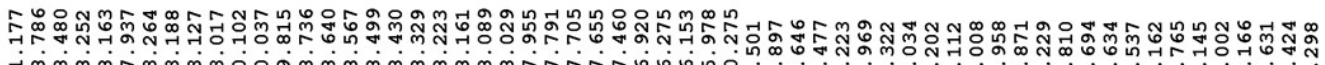

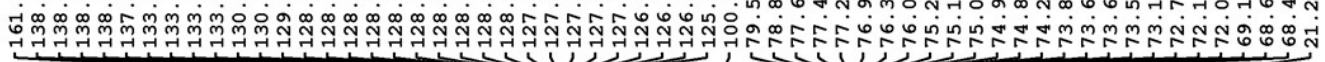

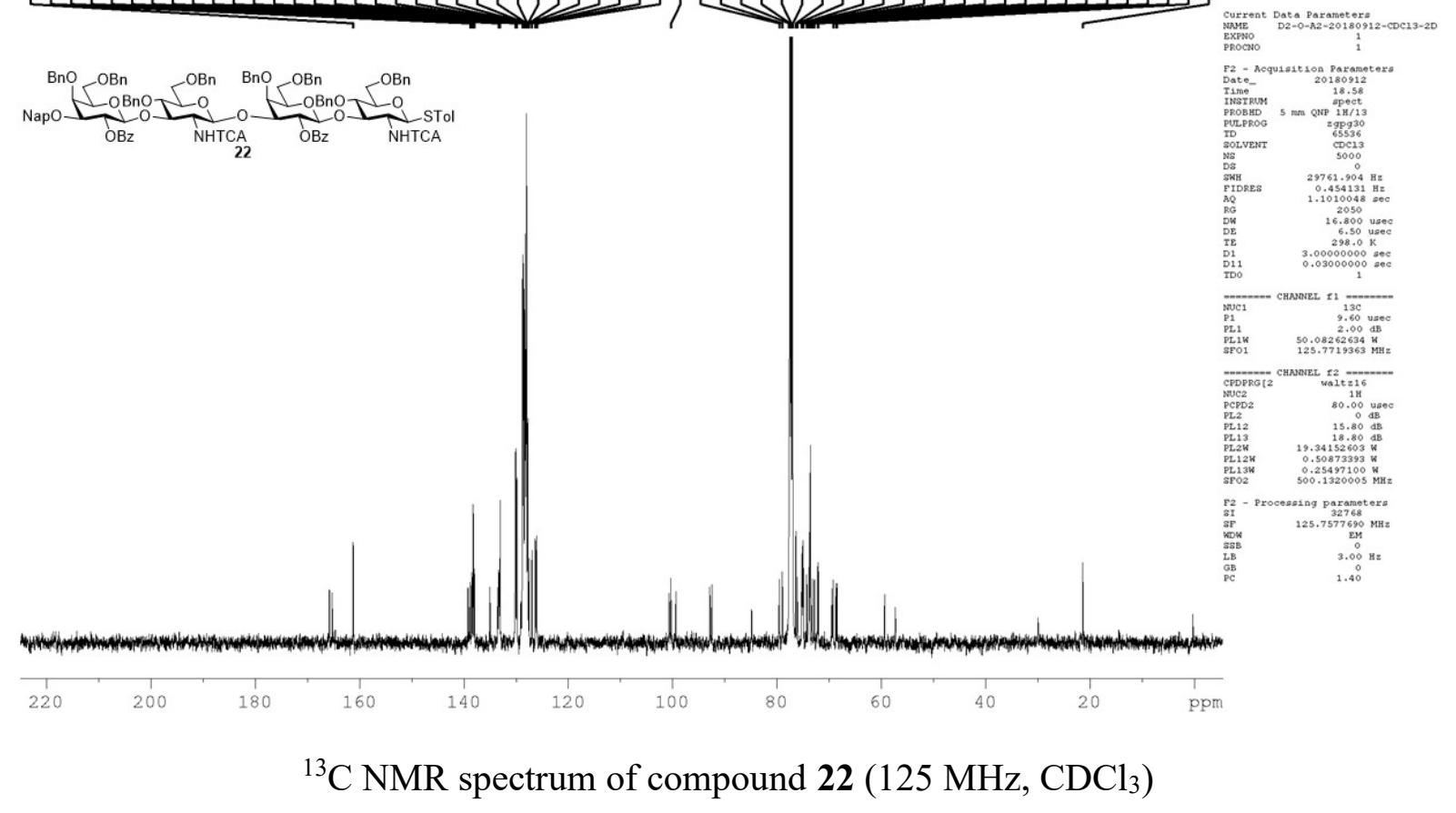




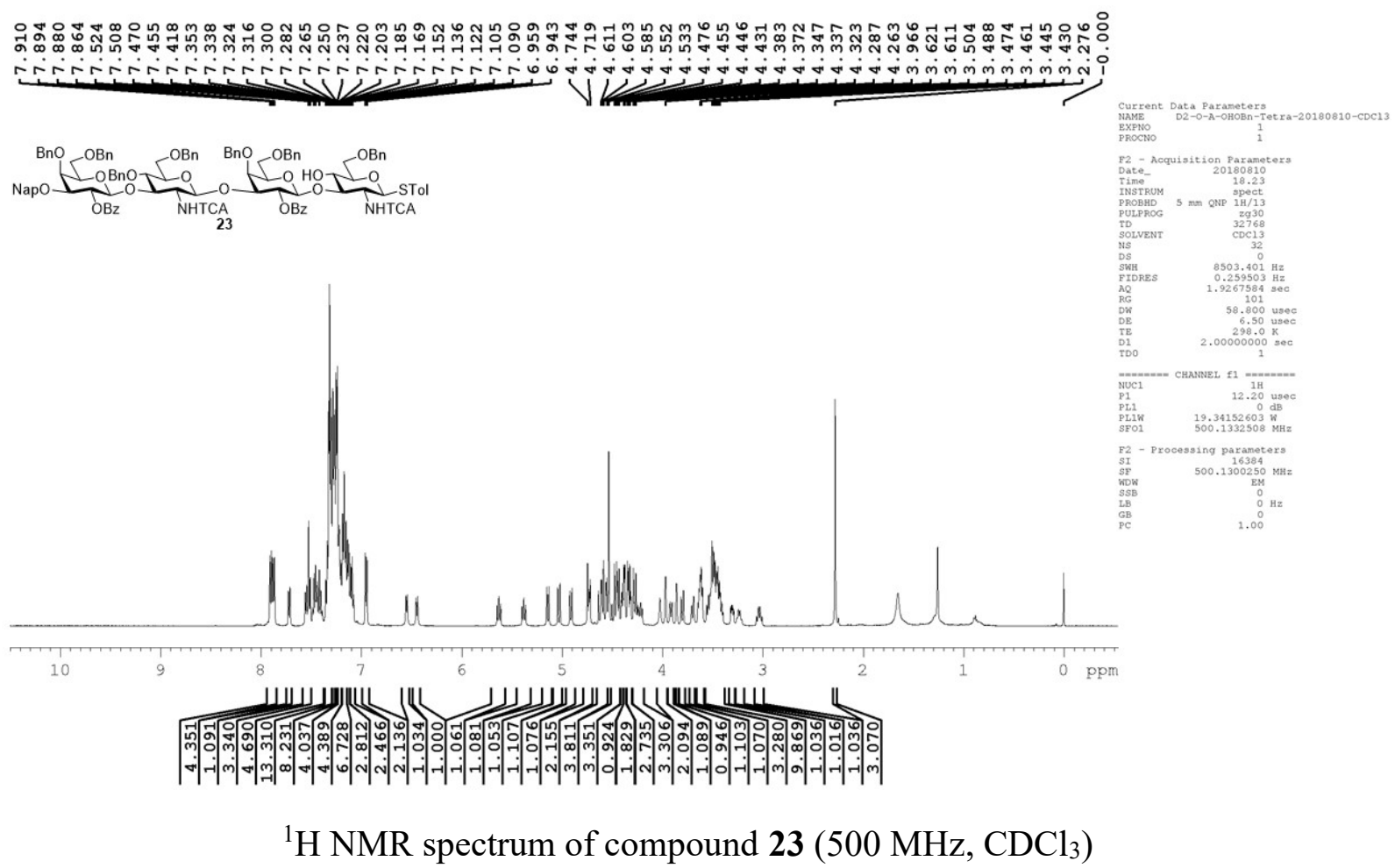

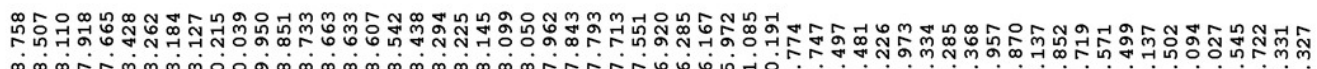
o

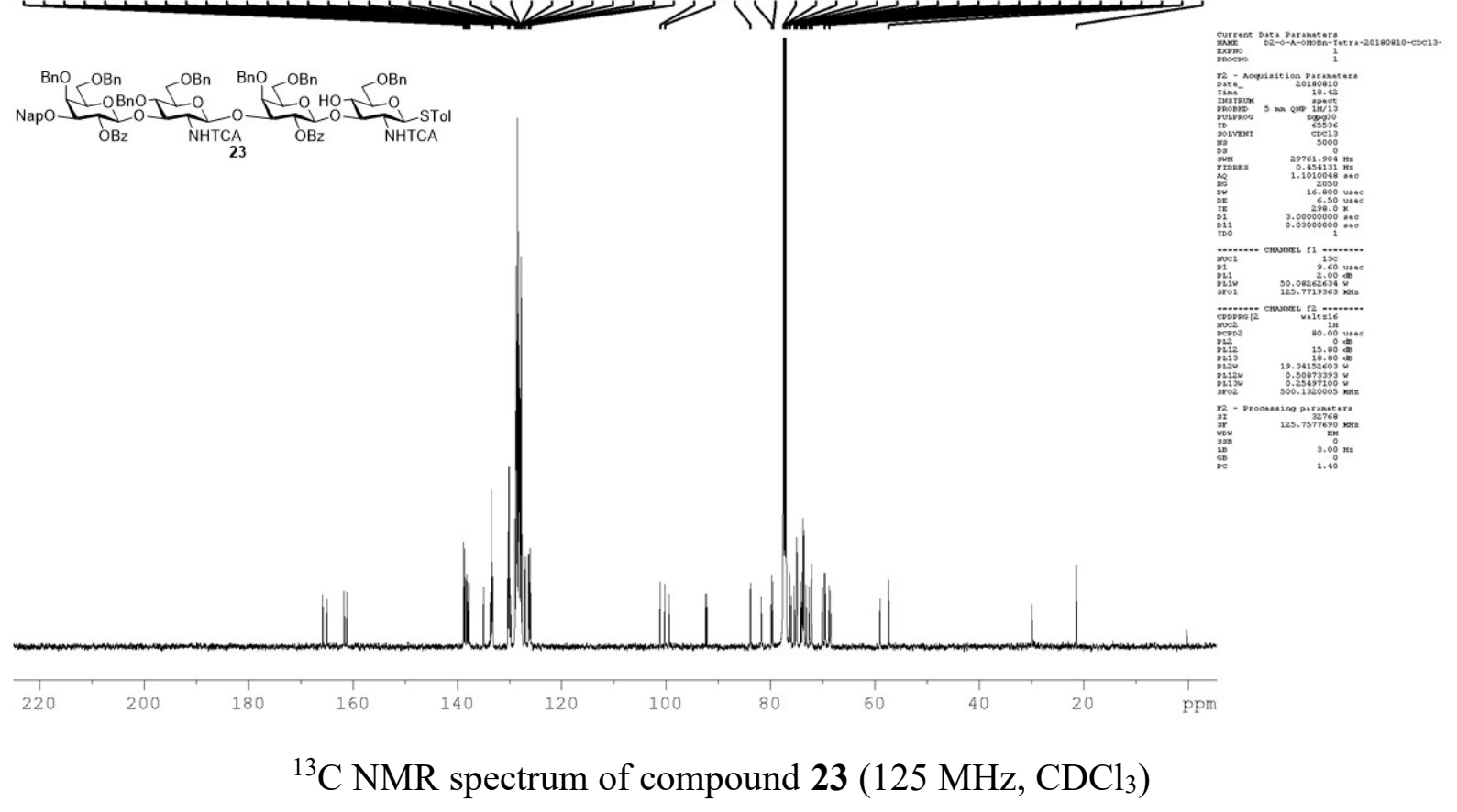



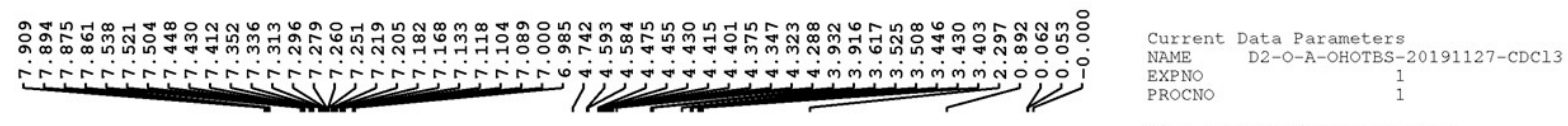

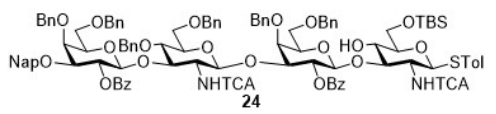

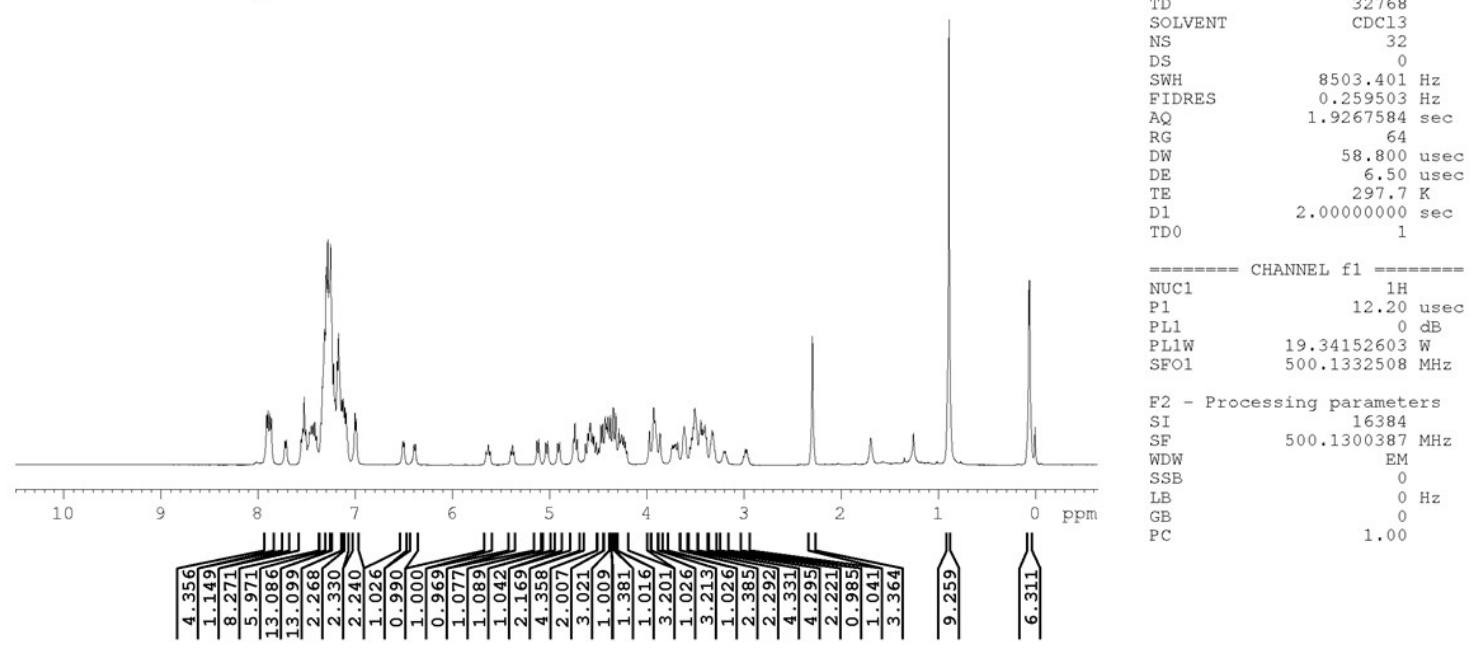

${ }^{1} \mathrm{H}$ NMR spectrum of compound $24\left(500 \mathrm{MHz}, \mathrm{CDCl}_{3}\right)$

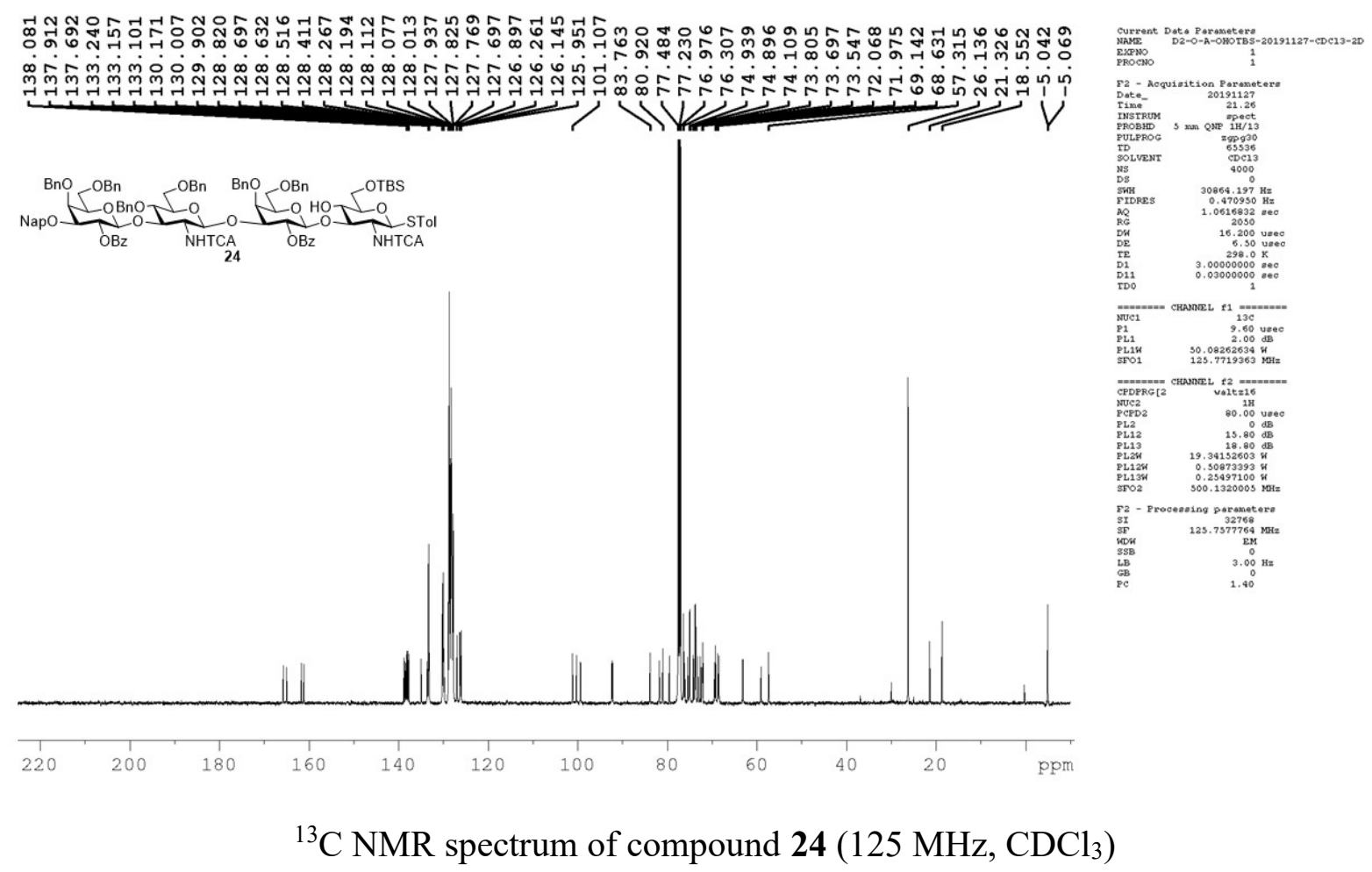




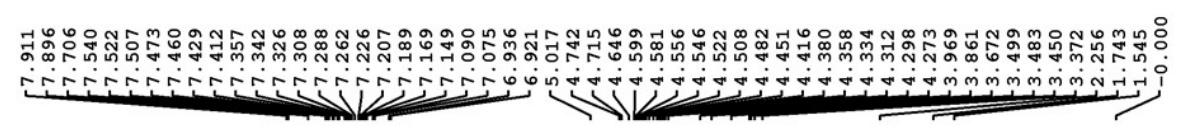

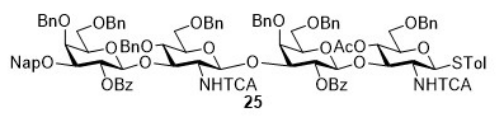
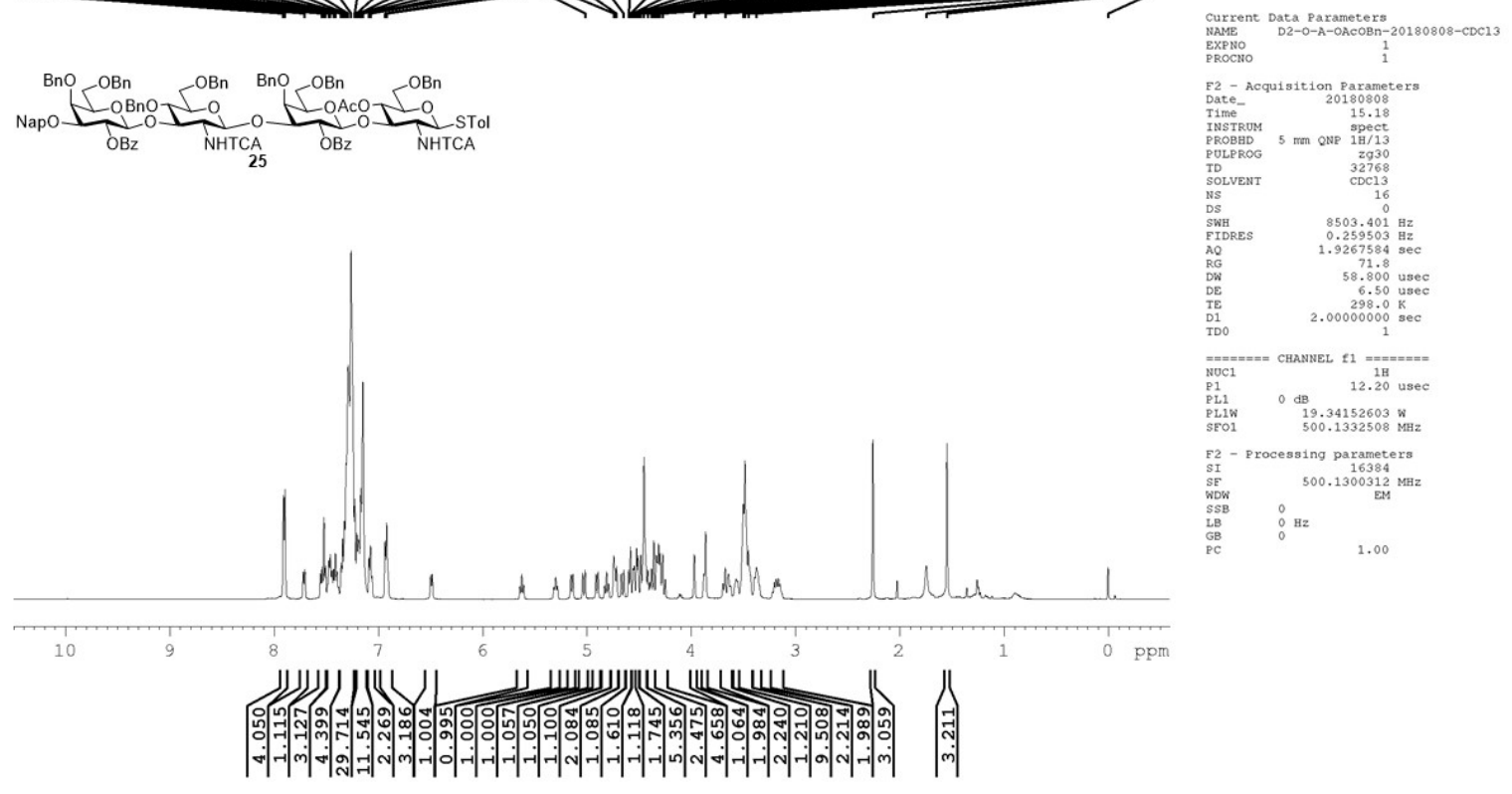

${ }^{1} \mathrm{H}$ NMR spectrum of compound $25\left(500 \mathrm{MHz}, \mathrm{CDCl}_{3}\right)$

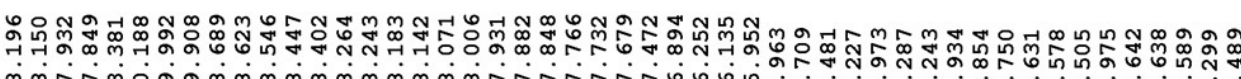

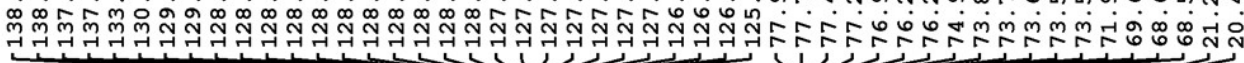

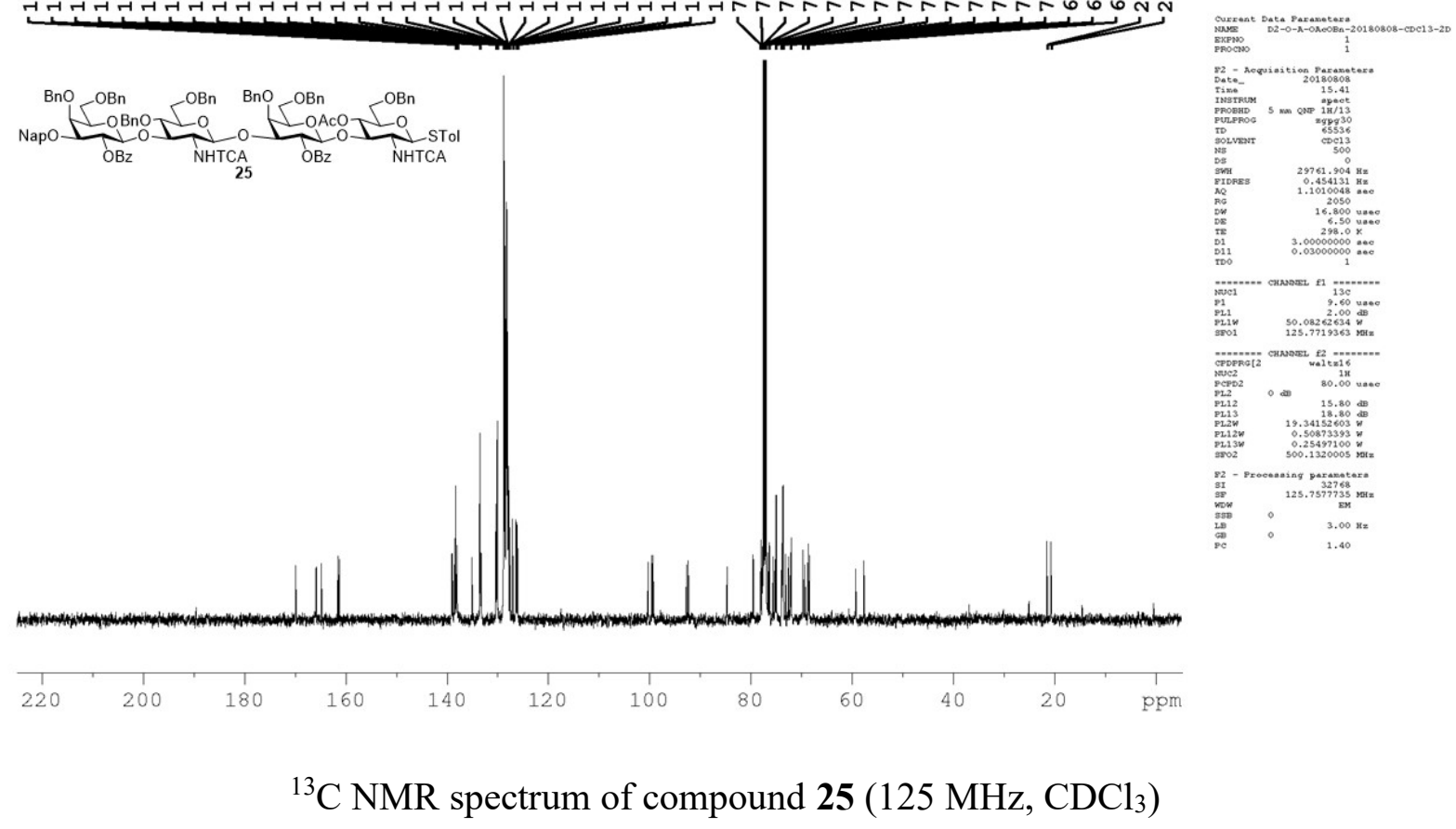



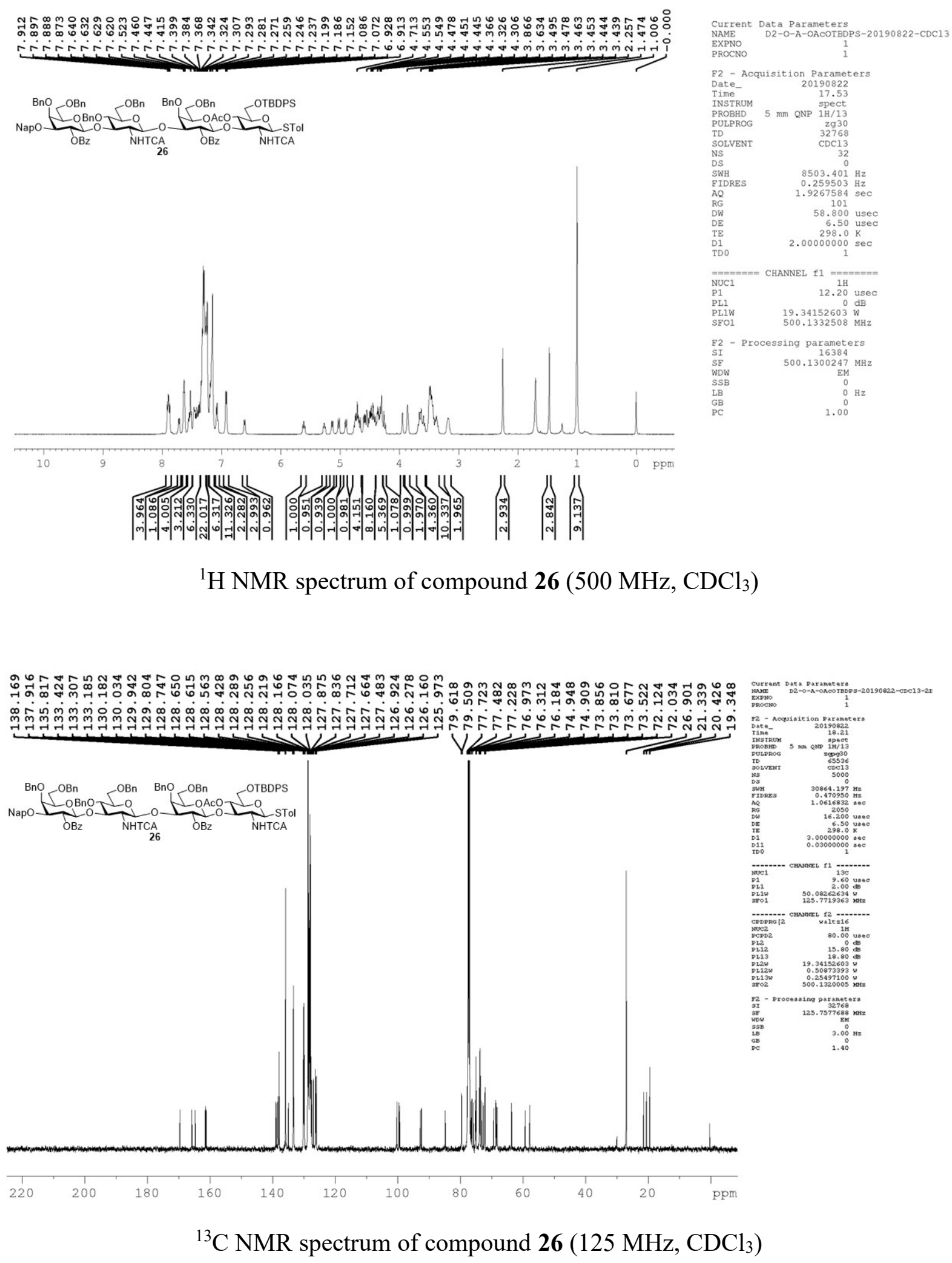

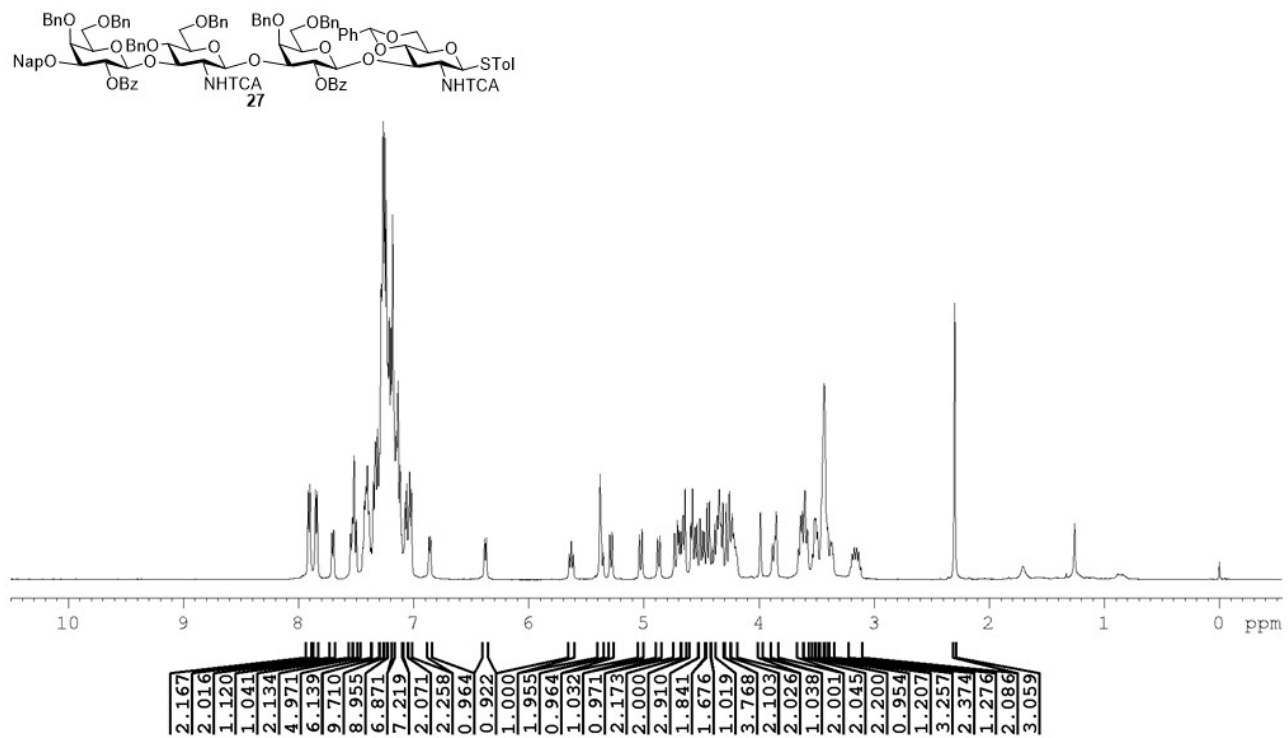

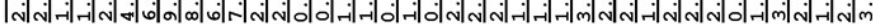

${ }^{1} \mathrm{H}$ NMR spectrum of compound $27\left(500 \mathrm{MHz}, \mathrm{CDCl}_{3}\right)$

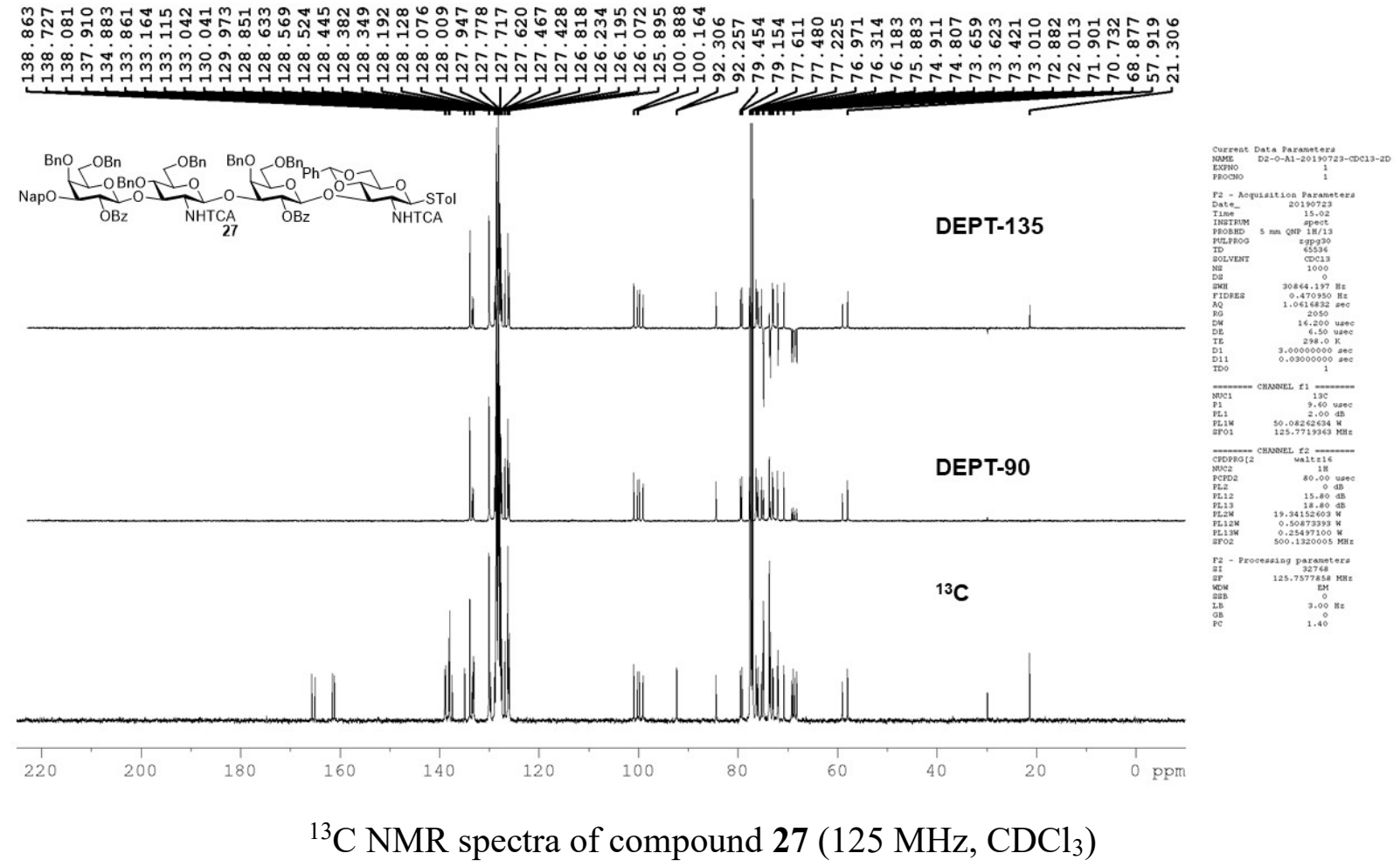




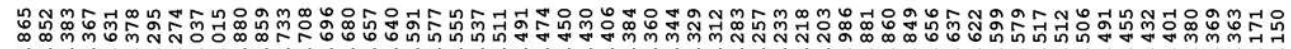

ソ

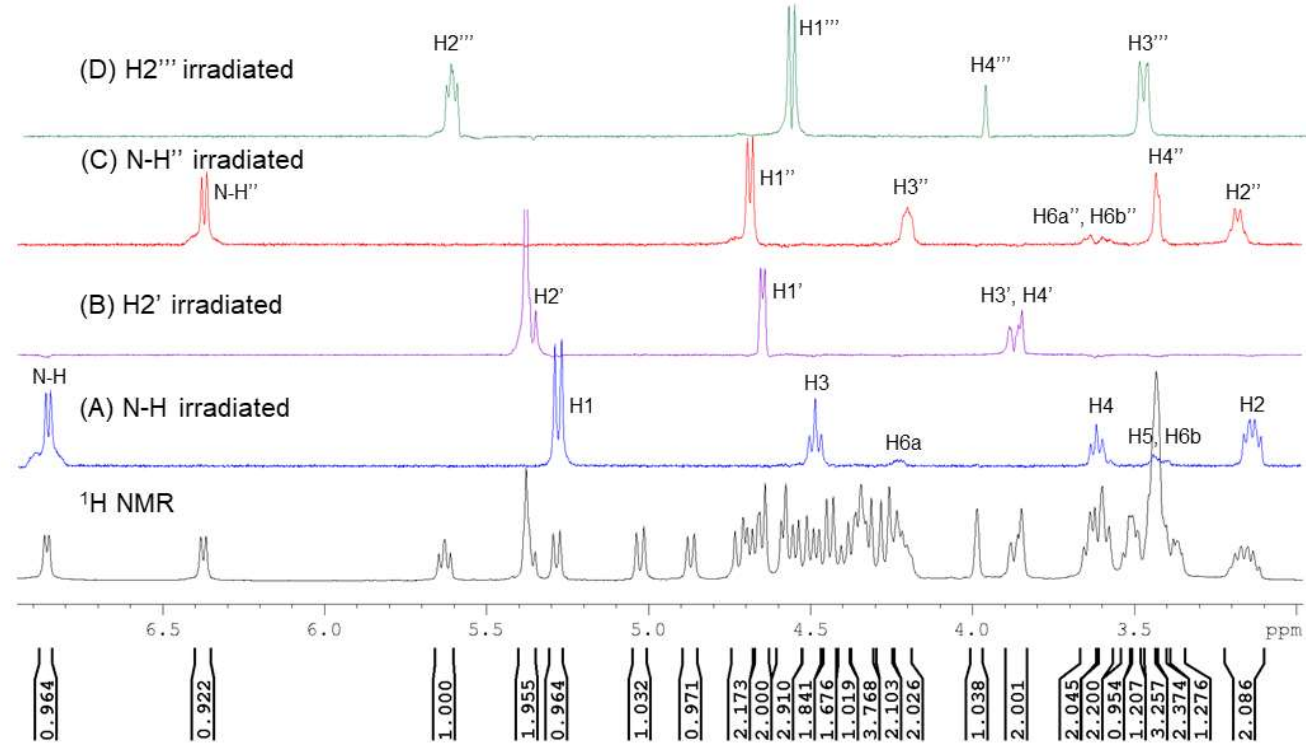

1D-Selective TOCSY NMR spectra of compound $27\left(500 \mathrm{MHz}, \mathrm{CDCl}_{3}\right)$

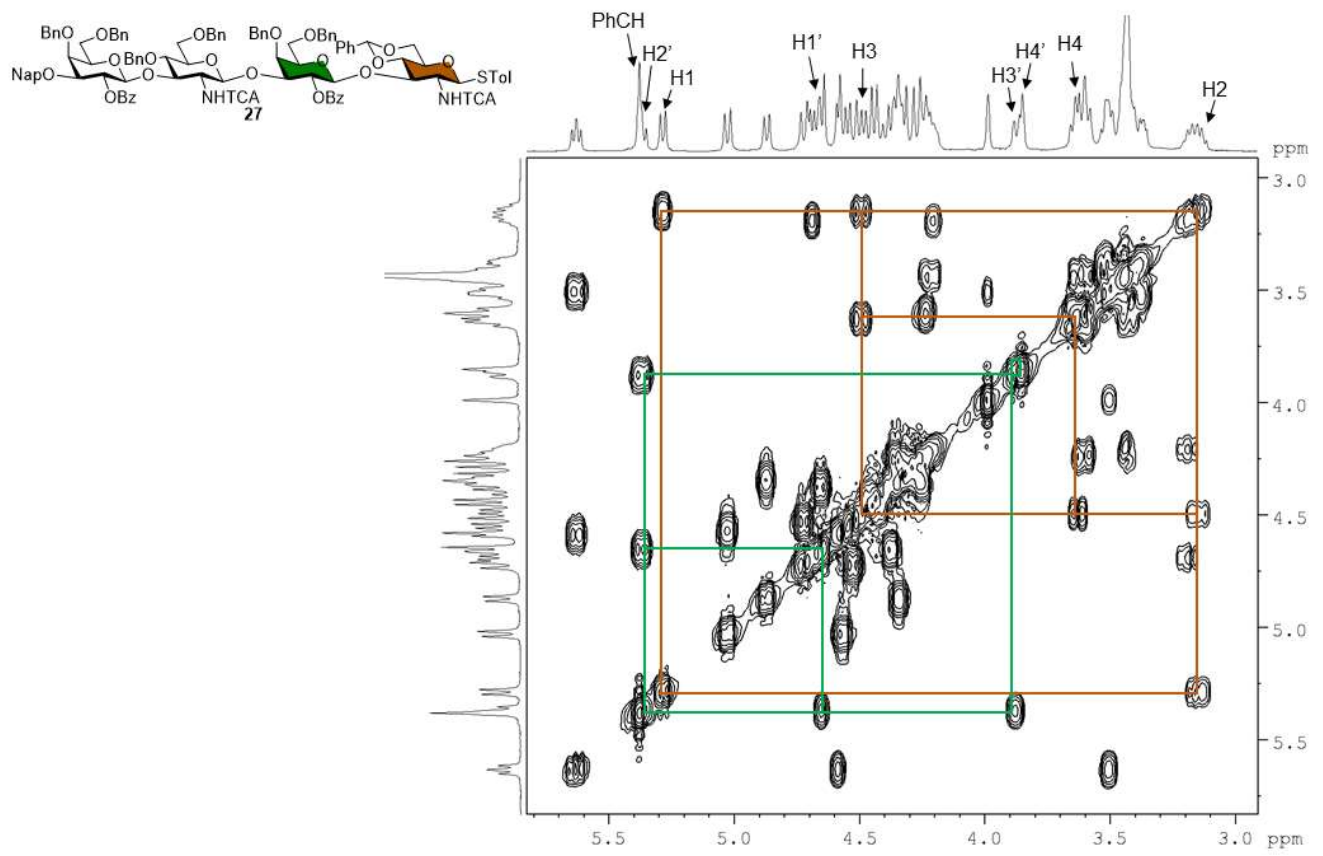

${ }^{1} \mathrm{H}-{ }^{1} \mathrm{H}$ 2D COSY NMR spectrum of compound $27\left(500 \mathrm{MHz}, \mathrm{CDCl}_{3}\right.$ ) 


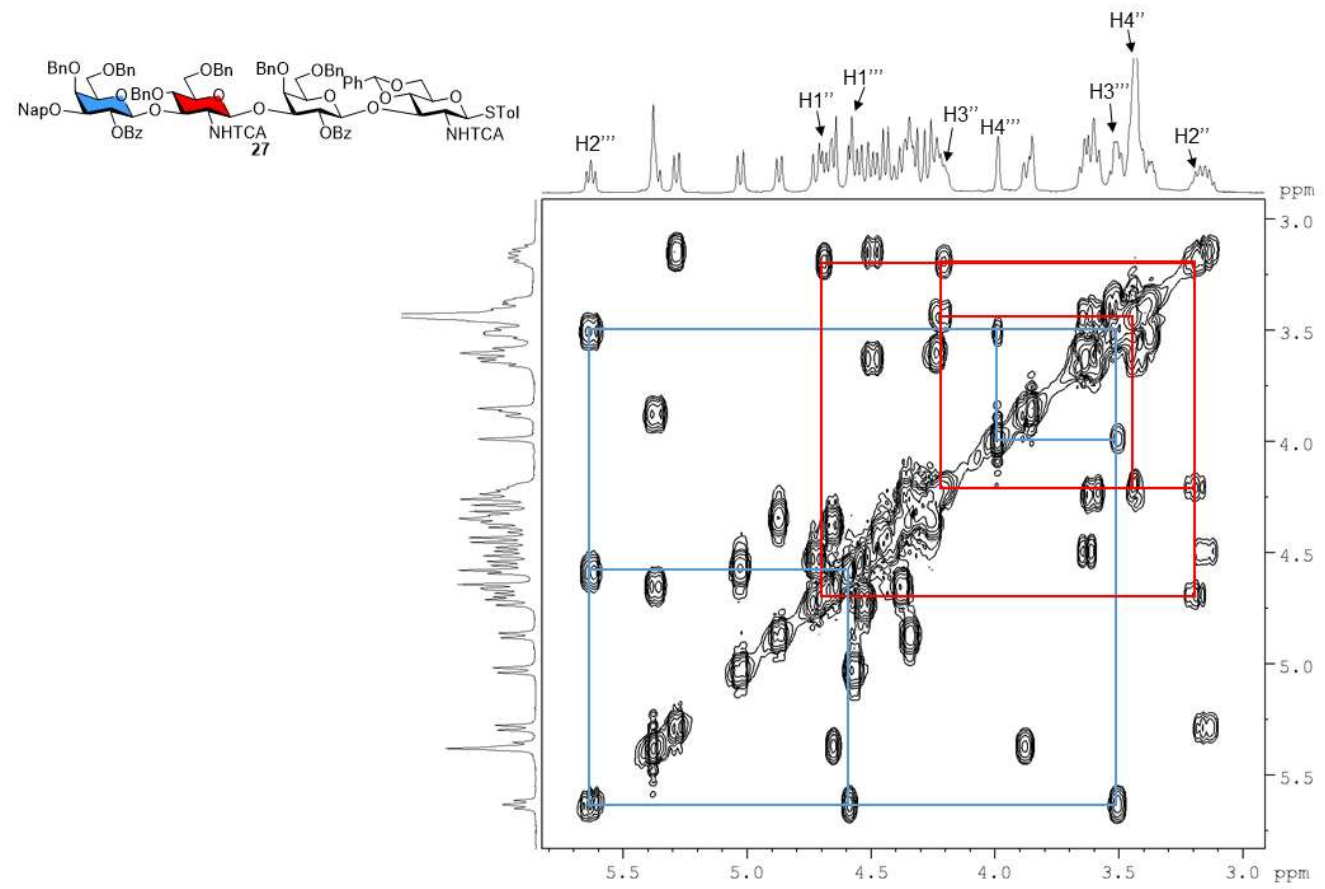

${ }^{1} \mathrm{H}-{ }^{1} \mathrm{H}$ 2D COSY NMR spectrum of compound $27\left(500 \mathrm{MHz}, \mathrm{CDCl}_{3}\right)$

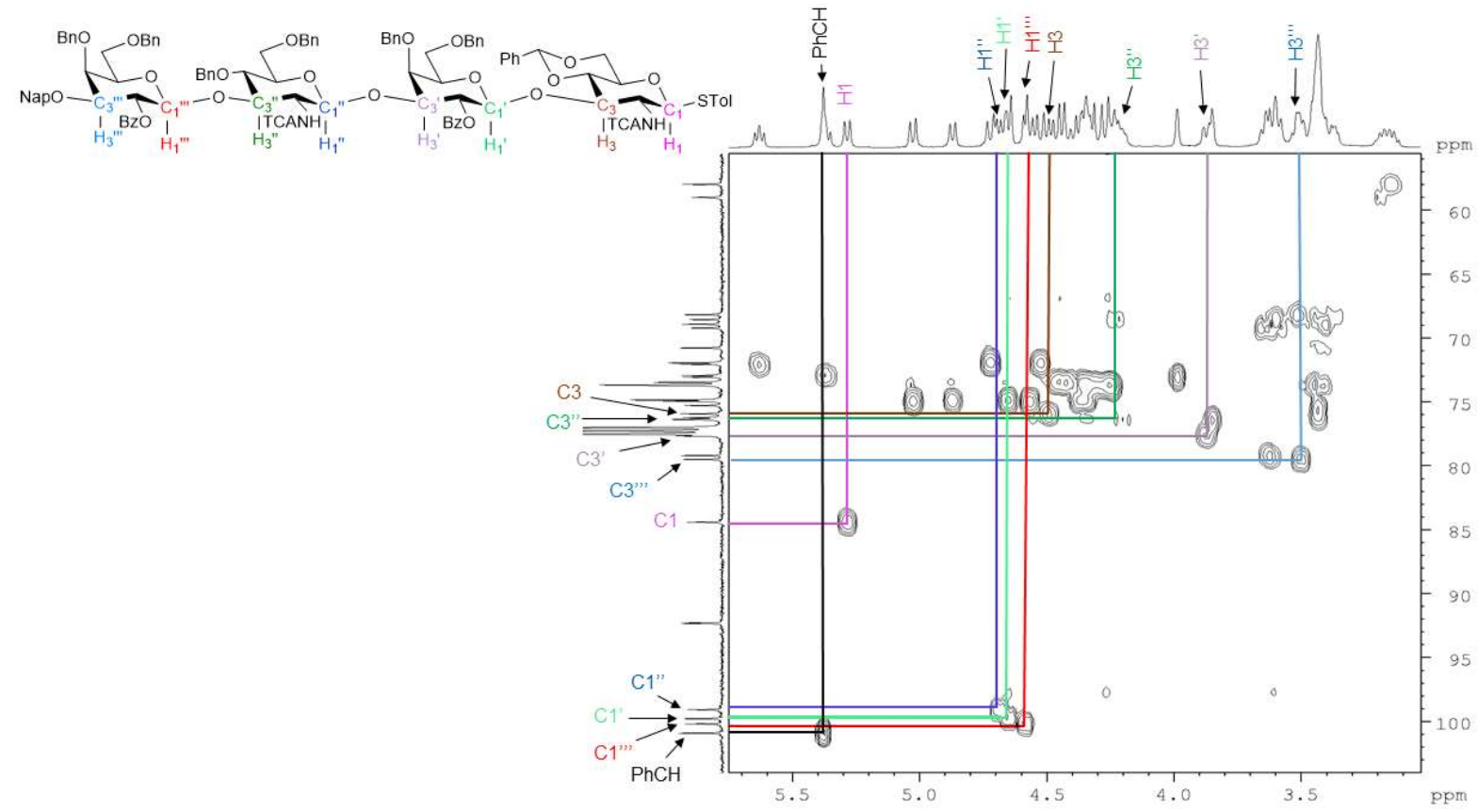

${ }^{1} \mathrm{H}-{ }^{13} \mathrm{C}$ 2D HMQC NMR spectrum of compound $27\left(500 / 125 \mathrm{MHz}, \mathrm{CDCl}_{3}\right)$ showing the selective correlations to identify $\mathrm{C} 1$ and $\mathrm{C} 3$ of Gal and GlcNTCA residues. 


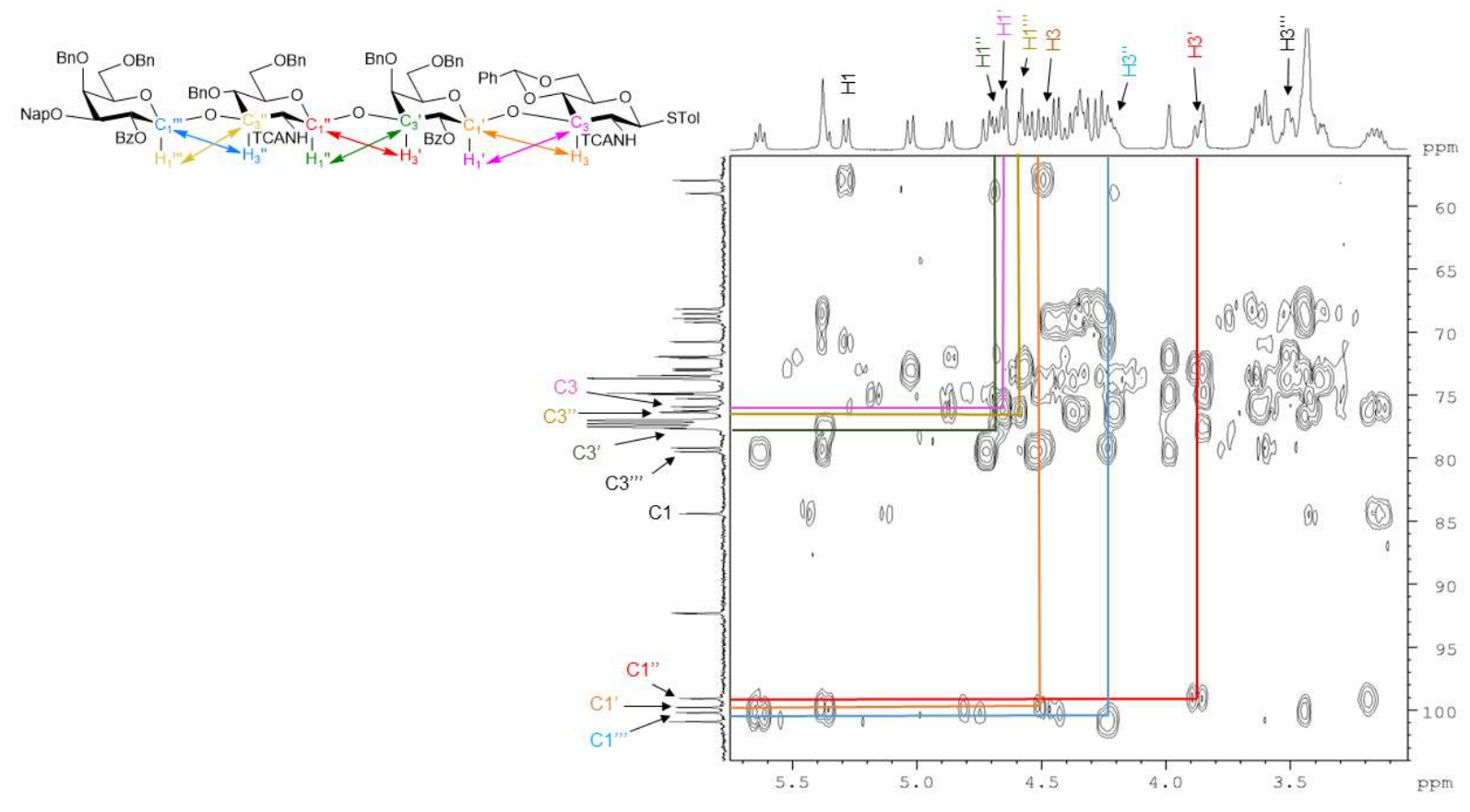

${ }^{1} \mathrm{H}-{ }^{13} \mathrm{C} 2 \mathrm{D}$ HMBC NMR spectrum of compound $27\left(500 / 125 \mathrm{MHz}, \mathrm{CDCl}_{3}\right)$ showing the selective correlations to confirm the $\beta$ 1-3 linkages. 


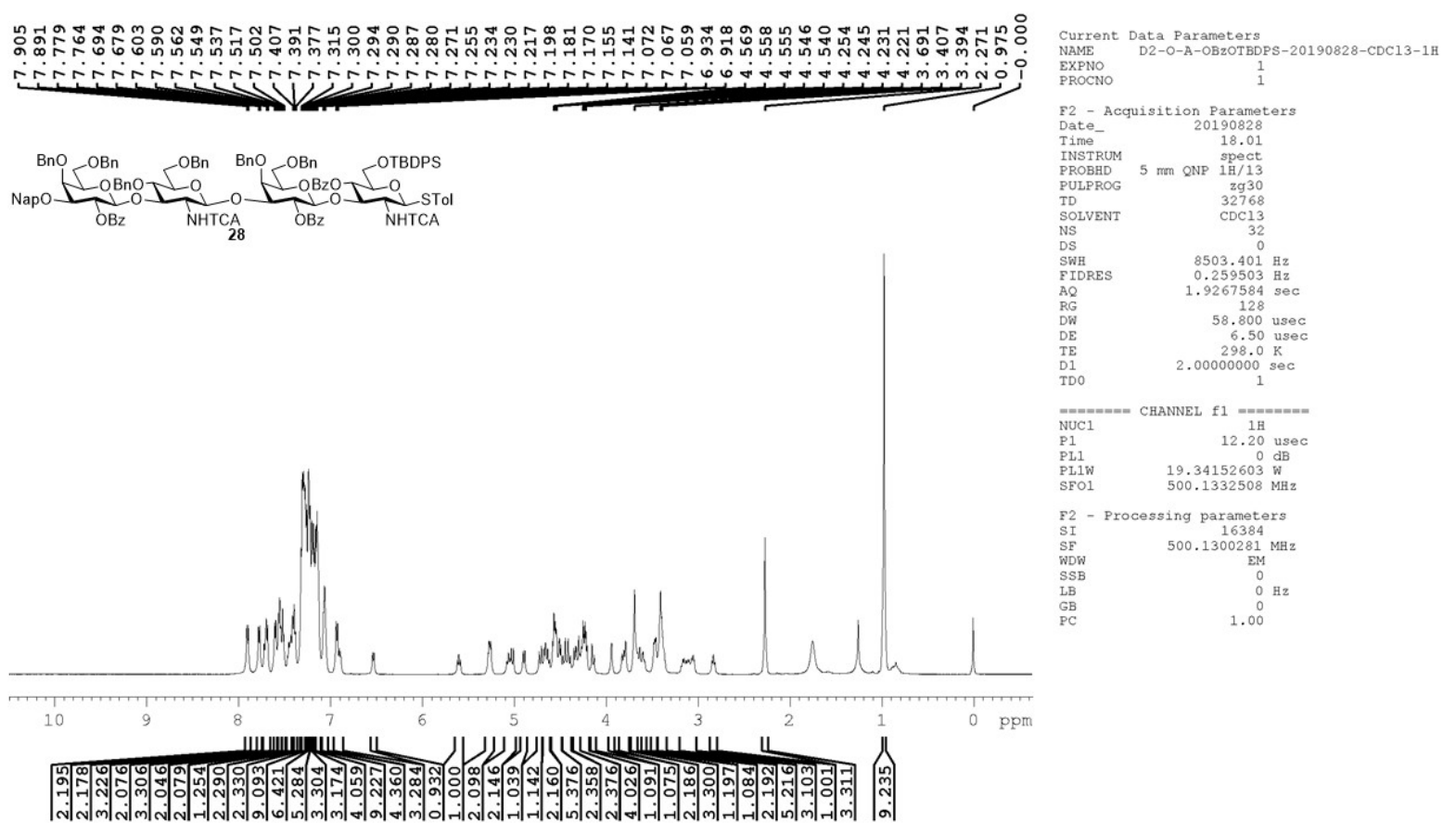

${ }^{1} \mathrm{H}$ NMR spectrum of compound $28\left(500 \mathrm{MHz}, \mathrm{CDCl}_{3}\right)$

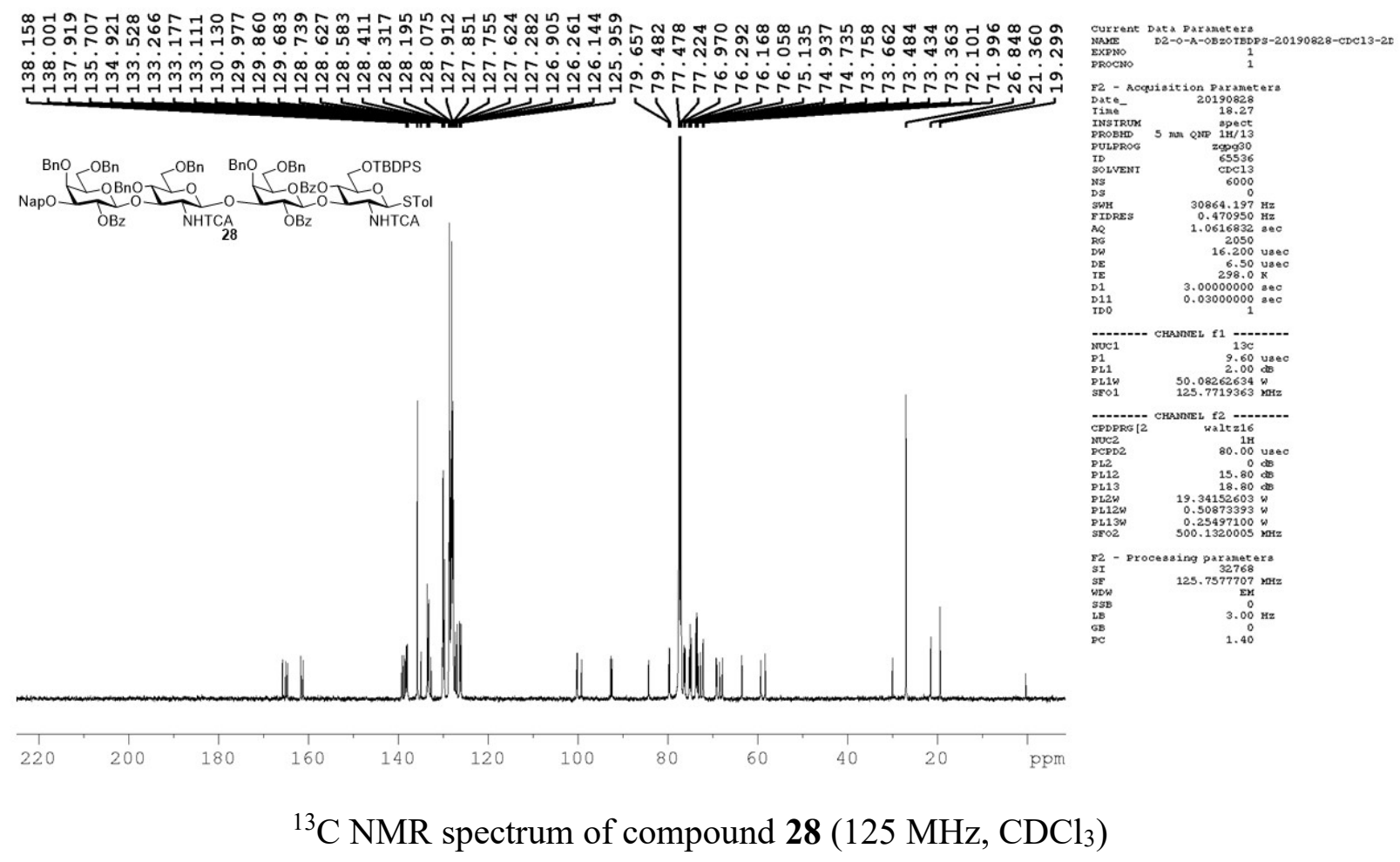



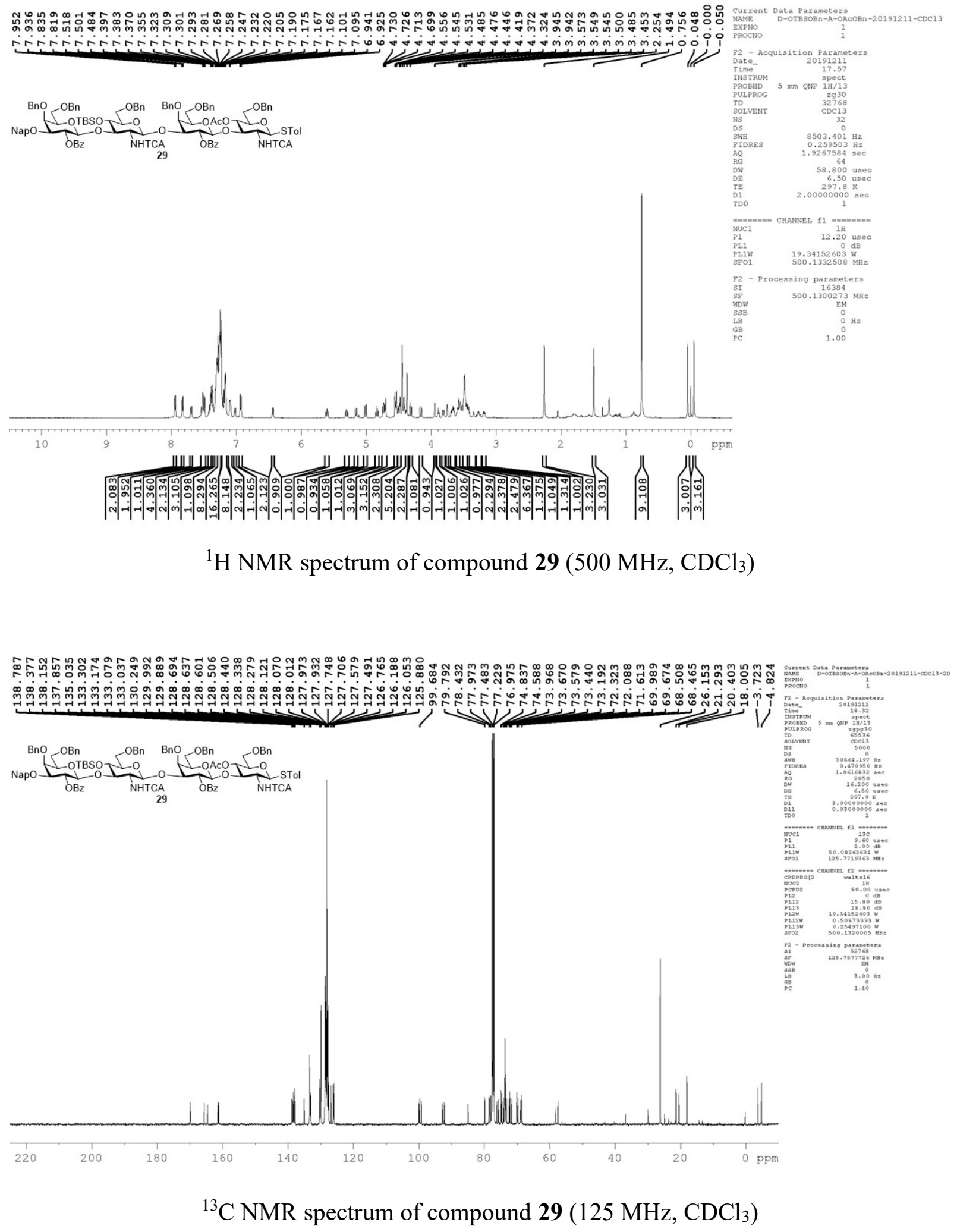
$\underbrace{\mathrm{O}}_{\mathrm{OBz}}$
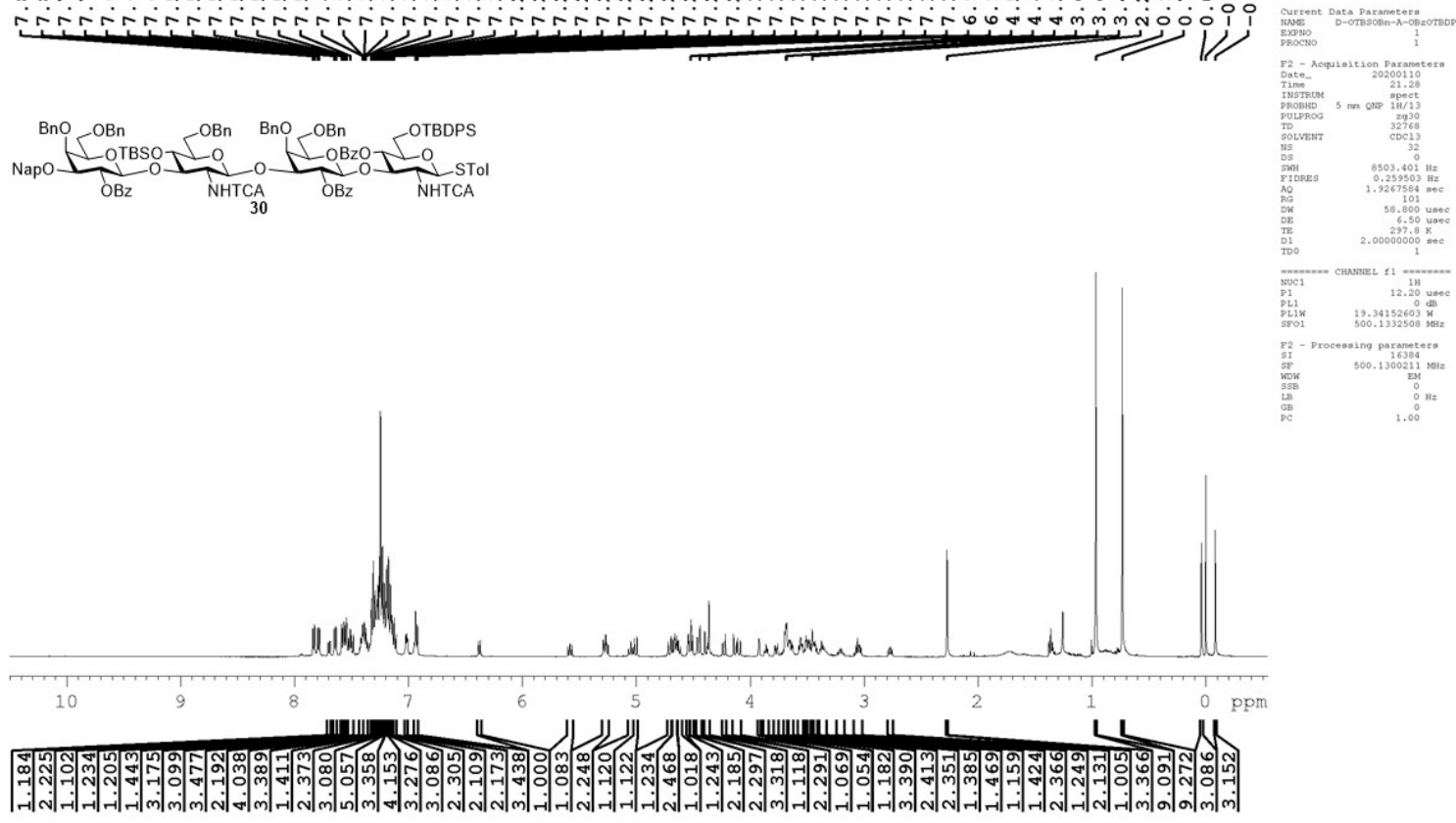

${ }^{1} \mathrm{H}$ NMR spectrum of compound $30\left(500 \mathrm{MHz}, \mathrm{CDCl}_{3}\right)$

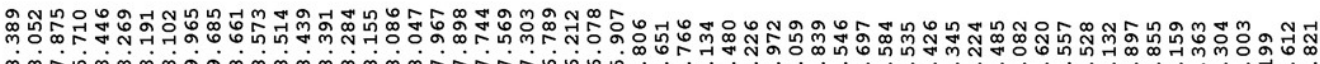

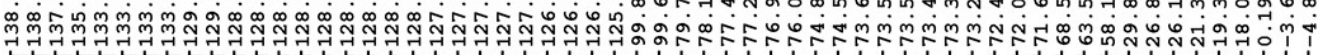
(1)

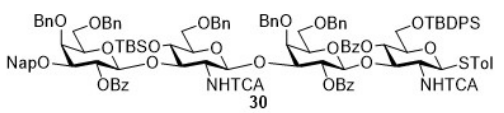
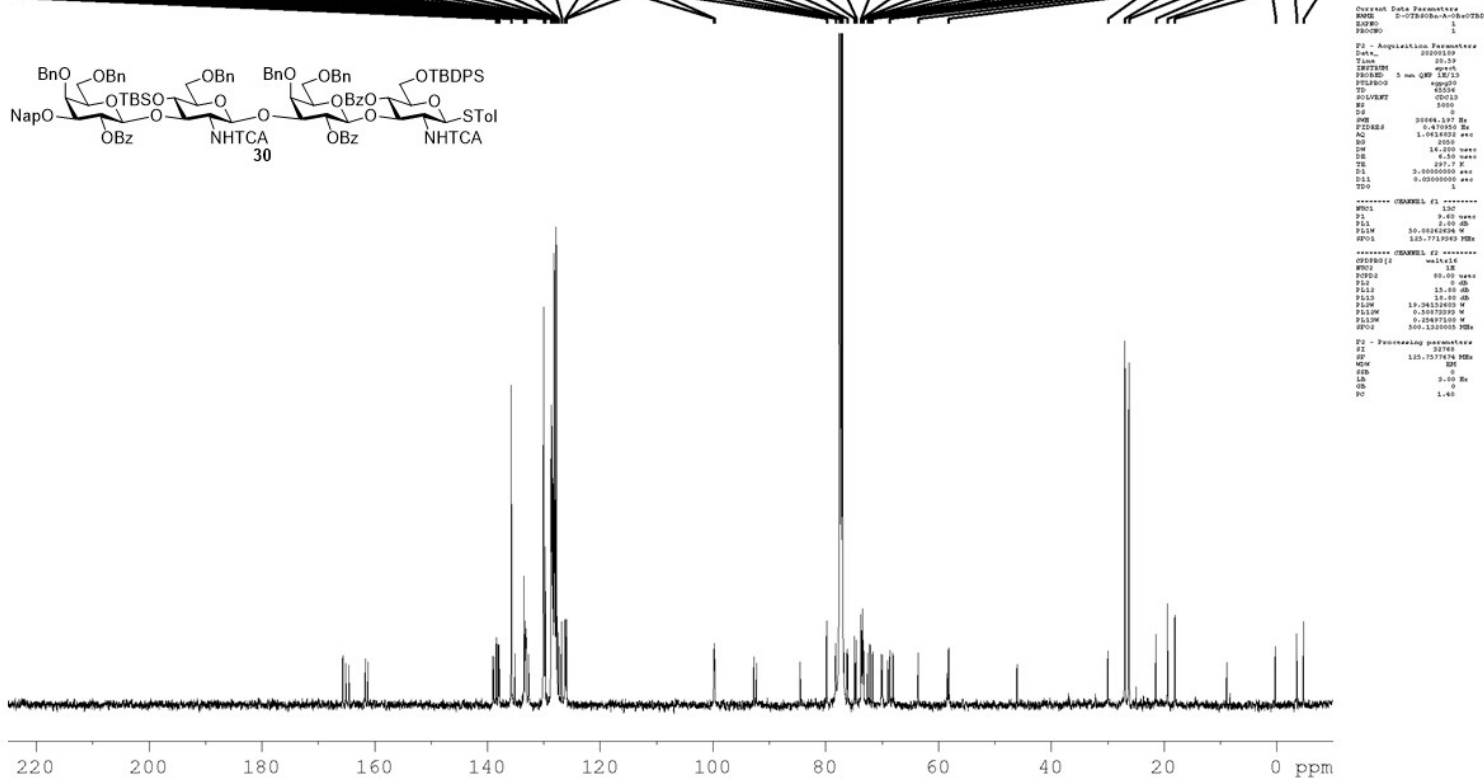

${ }^{13} \mathrm{C}$ NMR spectrum of compound $30\left(125 \mathrm{MHz}, \mathrm{CDCl}_{3}\right)$ 


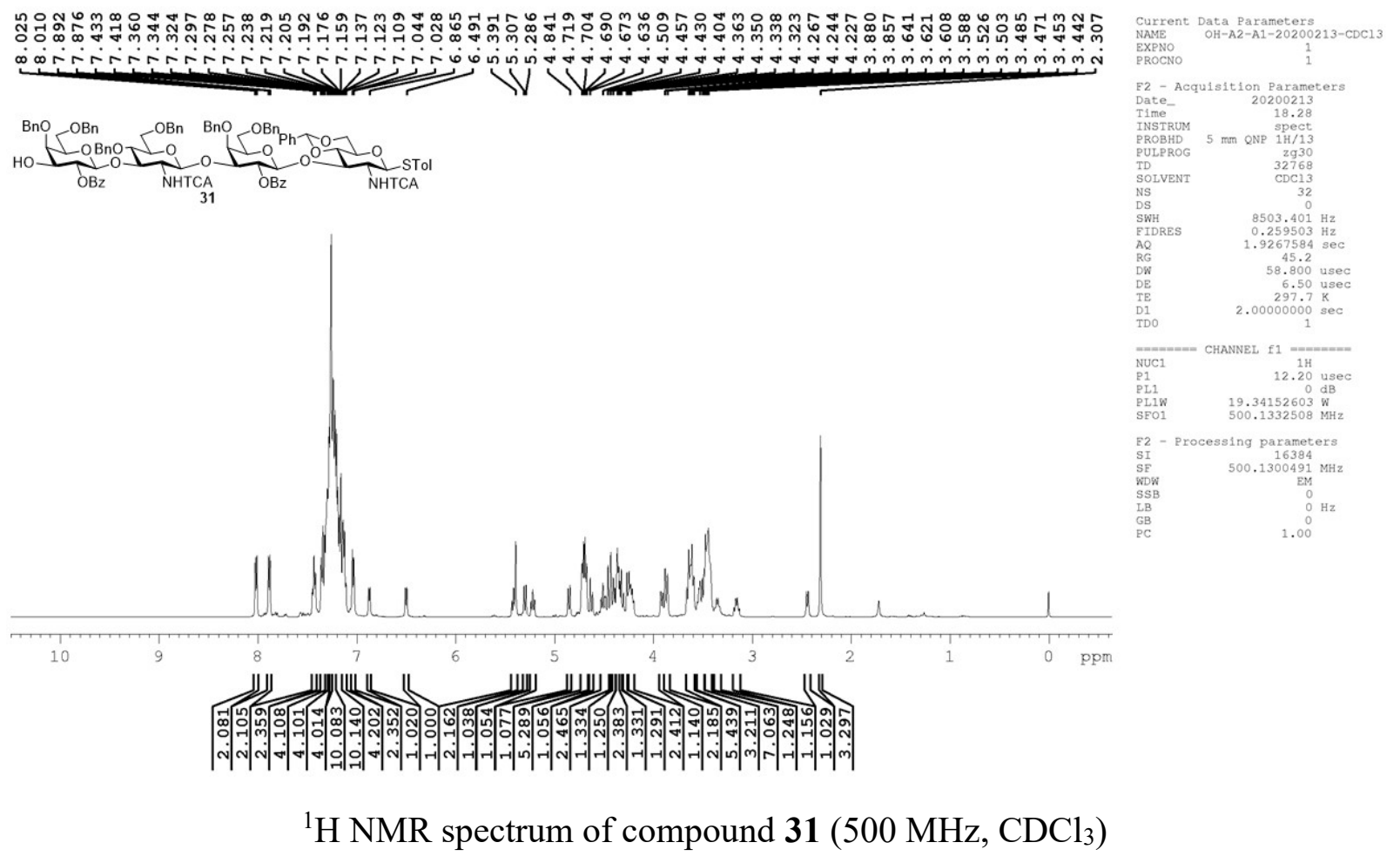

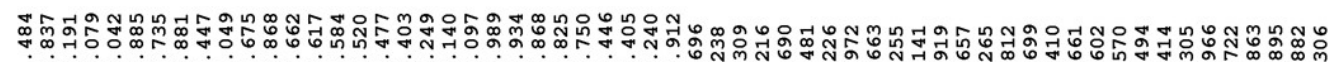

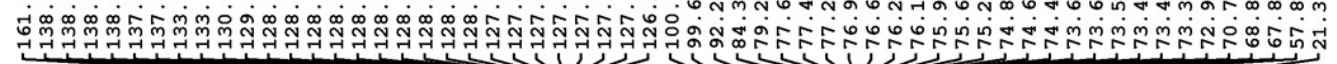
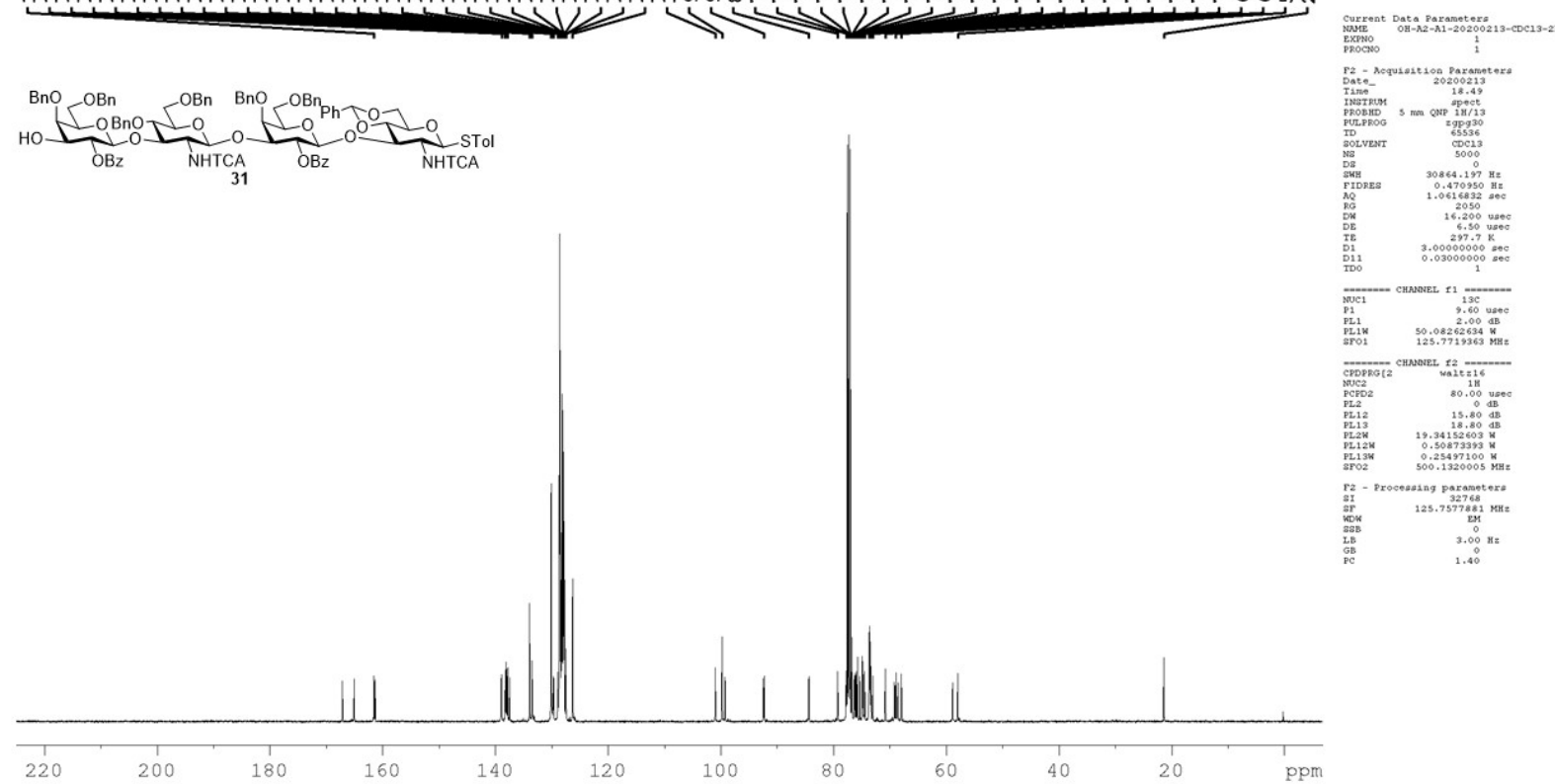

${ }^{13} \mathrm{C}$ NMR spectrum of compound $31\left(125 \mathrm{MHz}, \mathrm{CDCl}_{3}\right)$ 


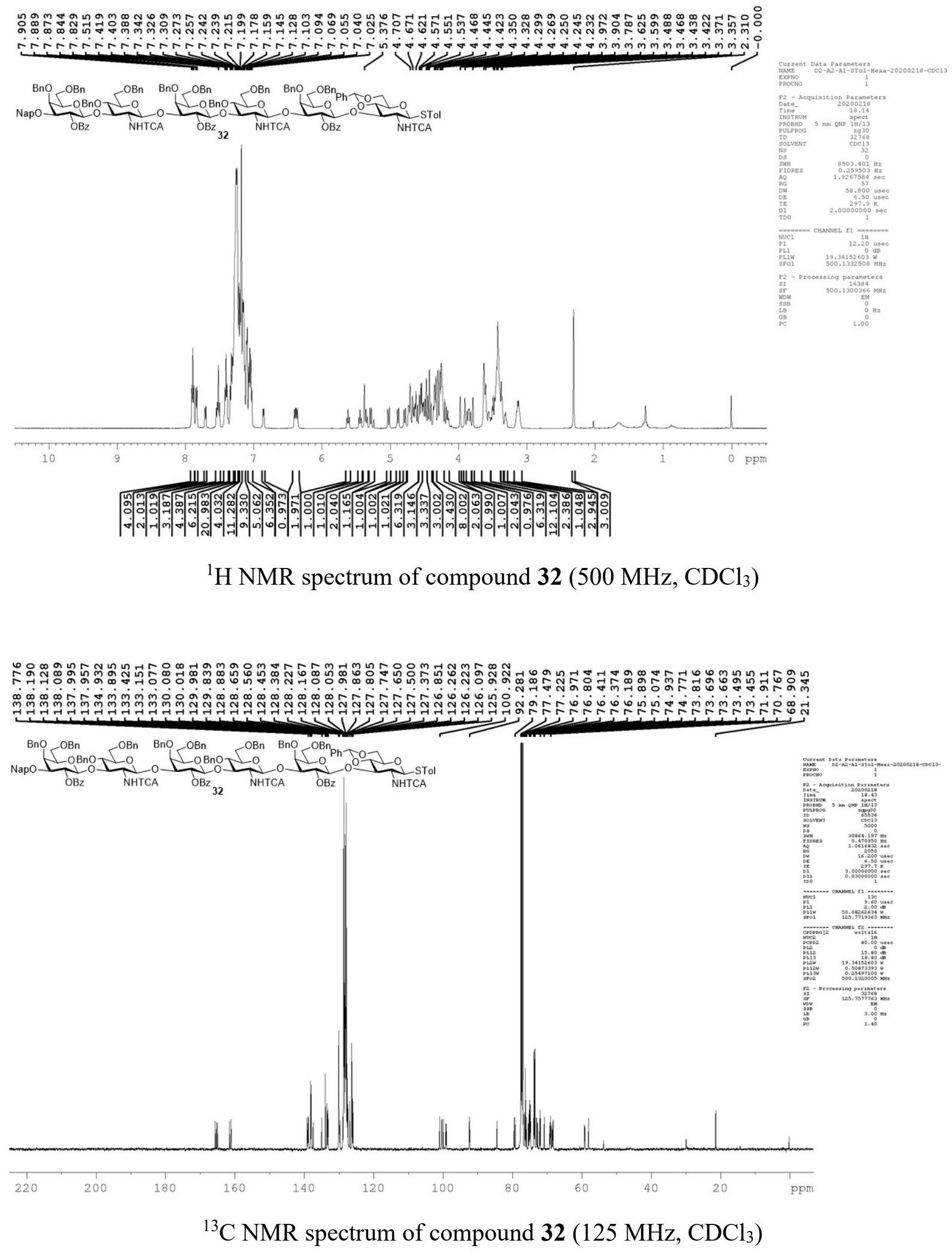




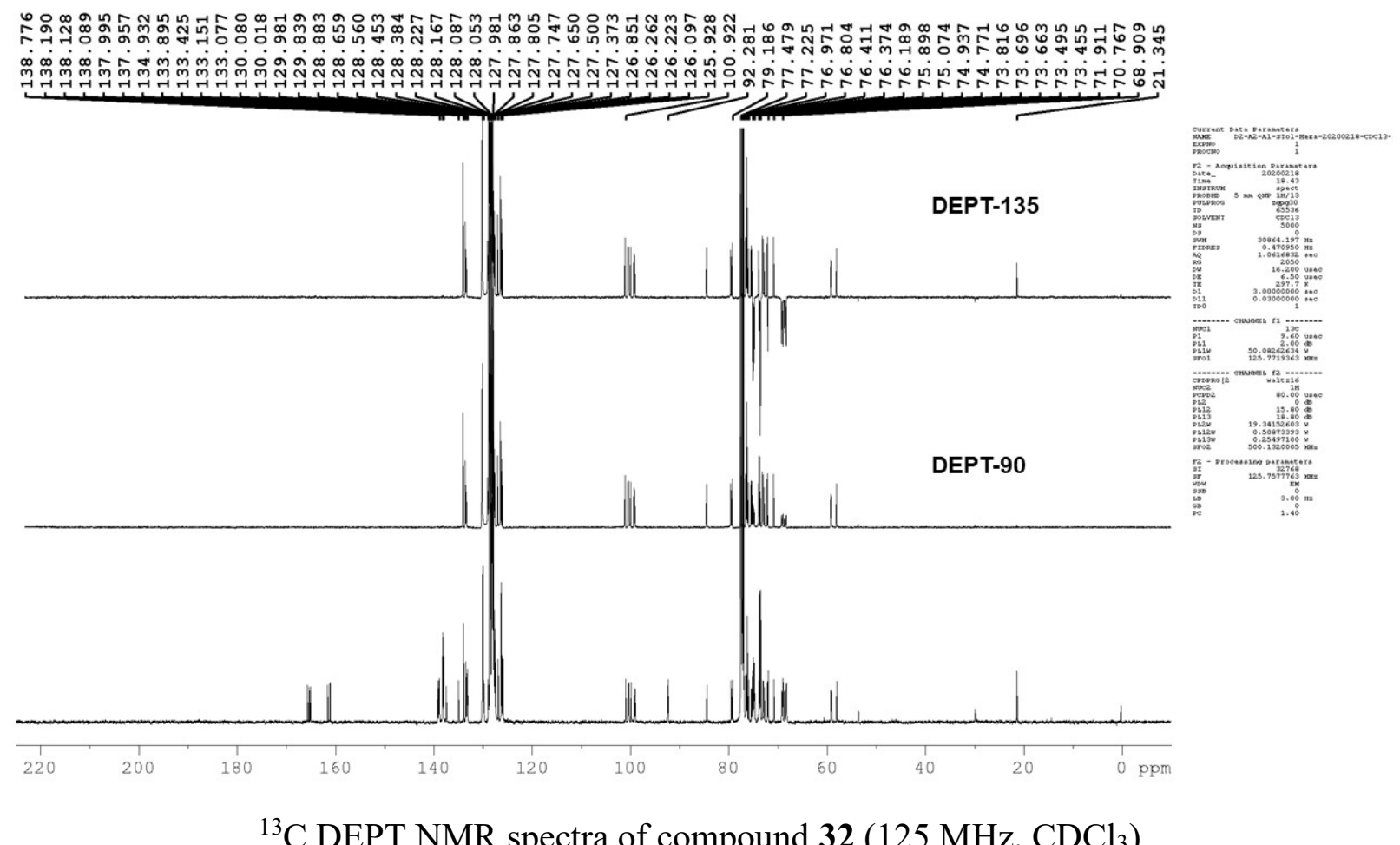

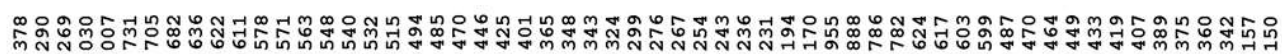

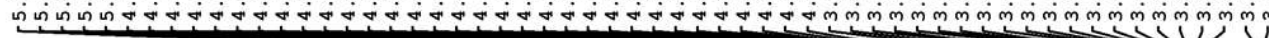

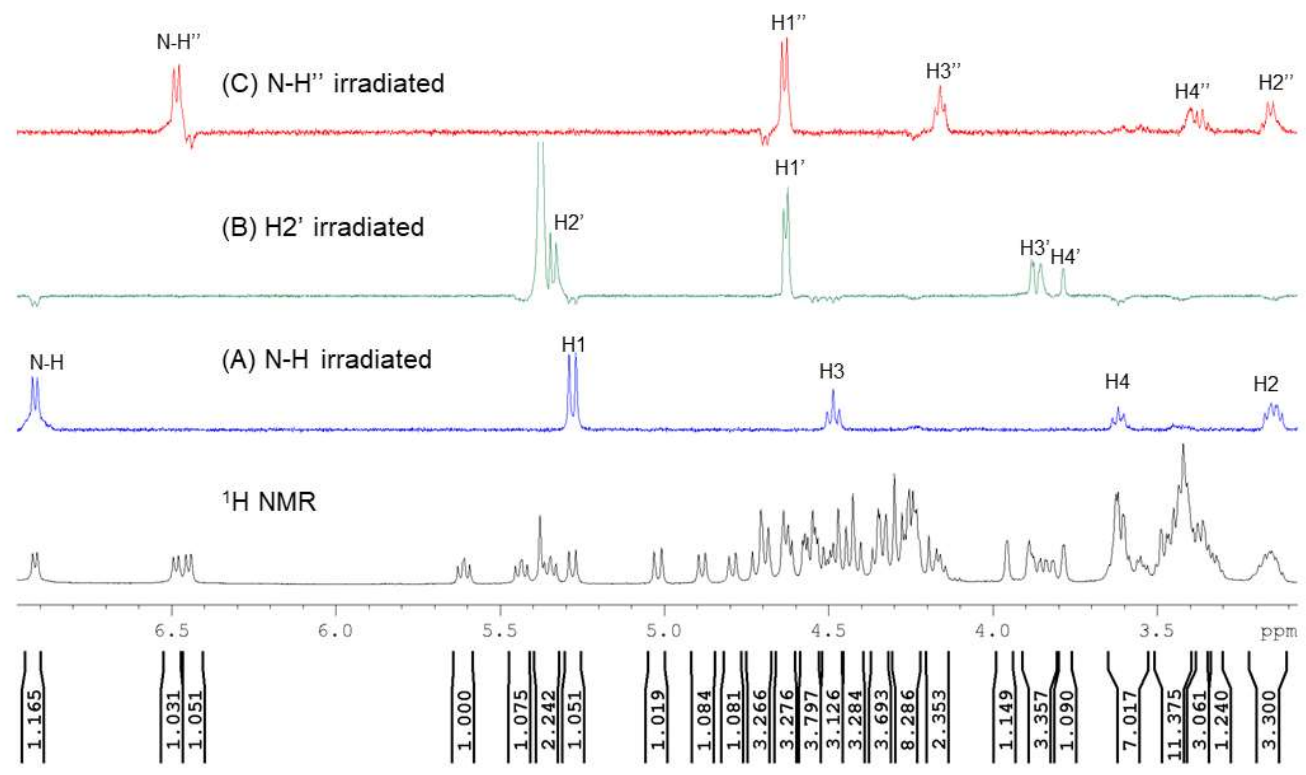

1D-Selective TOCSY NMR spectrum of compound $32\left(500 \mathrm{MHz}, \mathrm{CDCl}_{3}\right)$ 


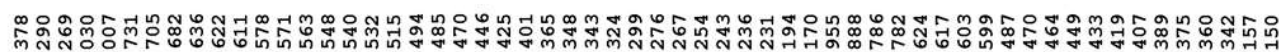

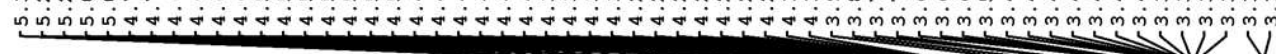

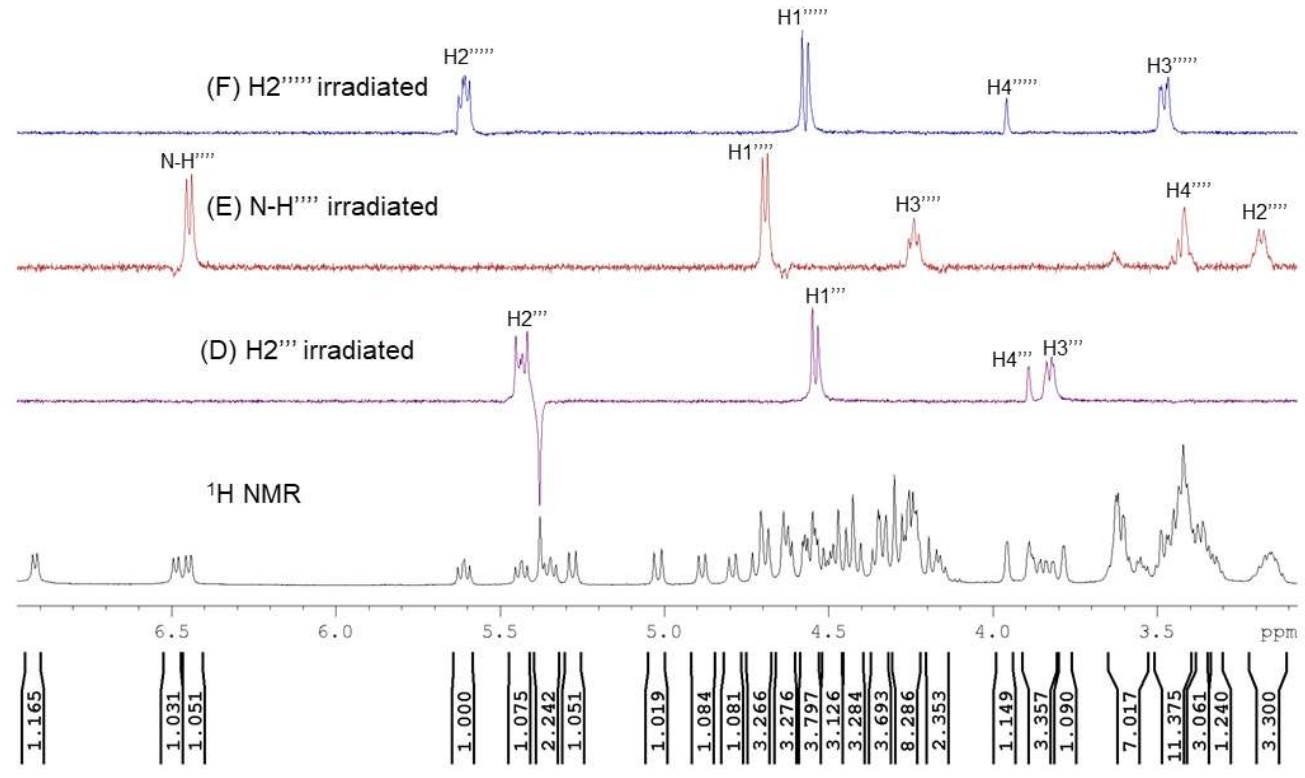

1D-Selective TOCSY NMR spectrum of compound $32\left(500 \mathrm{MHz}, \mathrm{CDCl}_{3}\right)$

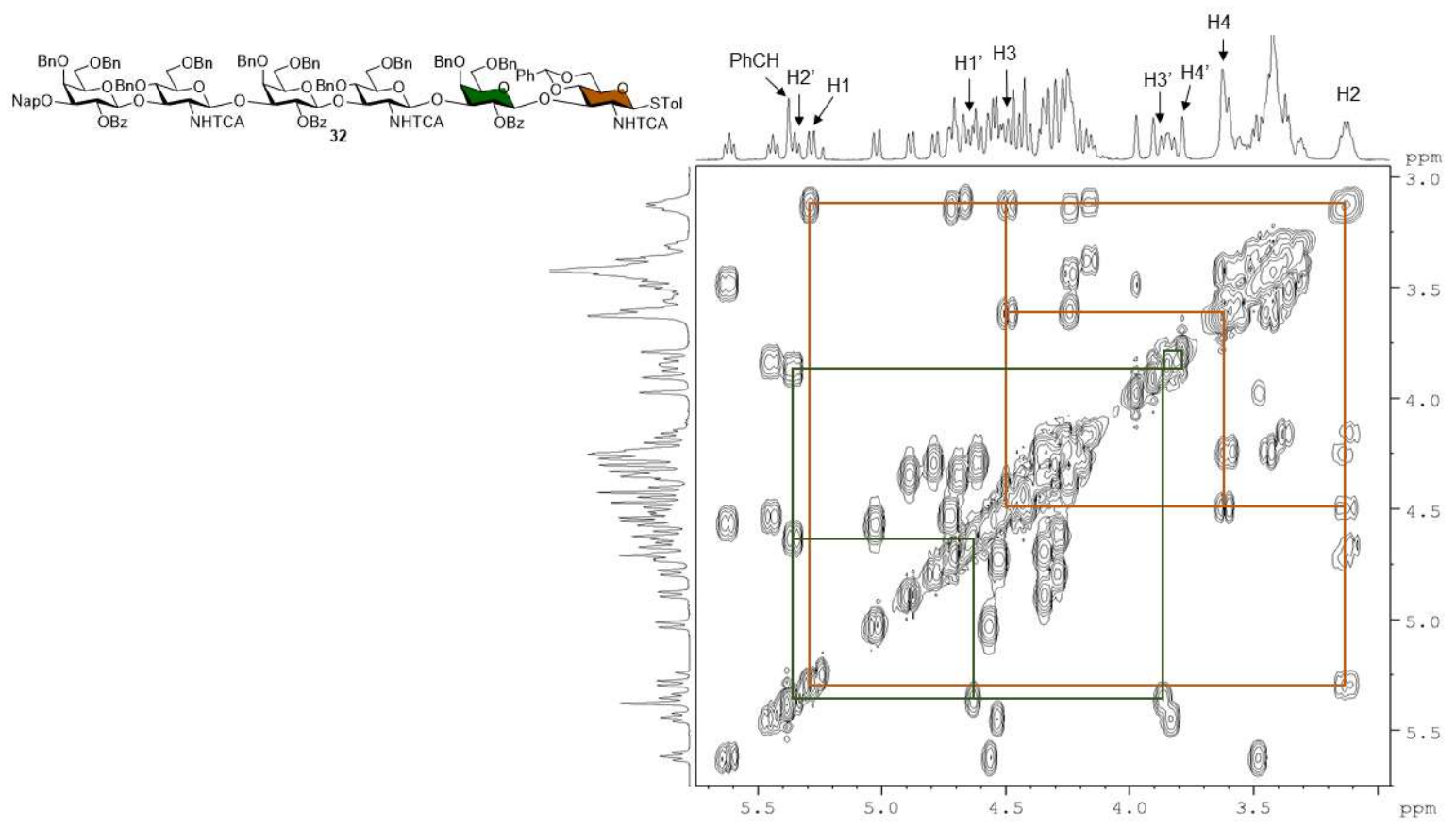

${ }^{1} \mathrm{H}-{ }^{1} \mathrm{H}$ 2D COSY NMR spectrum of compound $32\left(500 \mathrm{MHz}, \mathrm{CDCl}_{3}\right)$ 


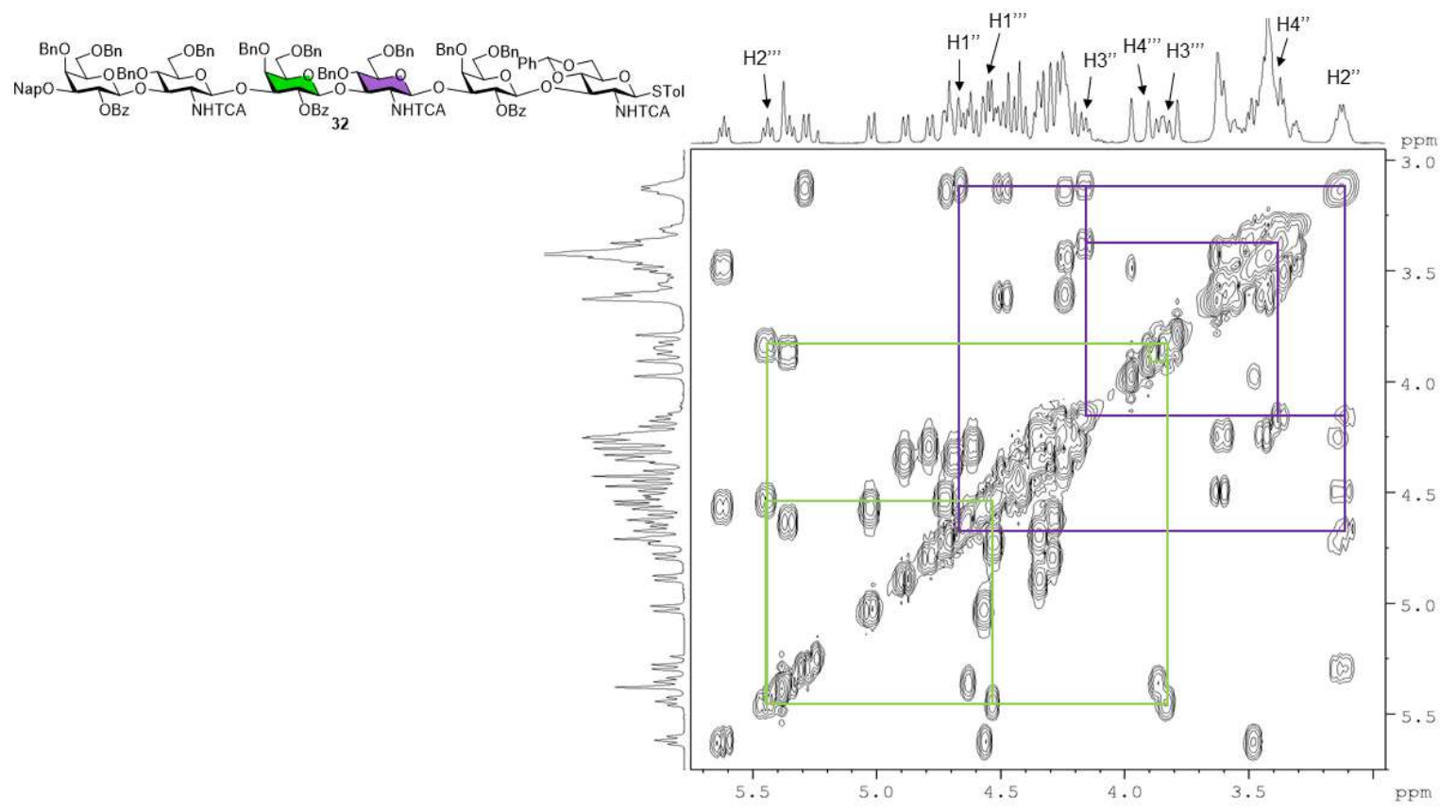

${ }^{1} \mathrm{H}-{ }^{1} \mathrm{H}$ 2D COSY NMR spectrum of compound $32\left(500 \mathrm{MHz}, \mathrm{CDCl}_{3}\right)$

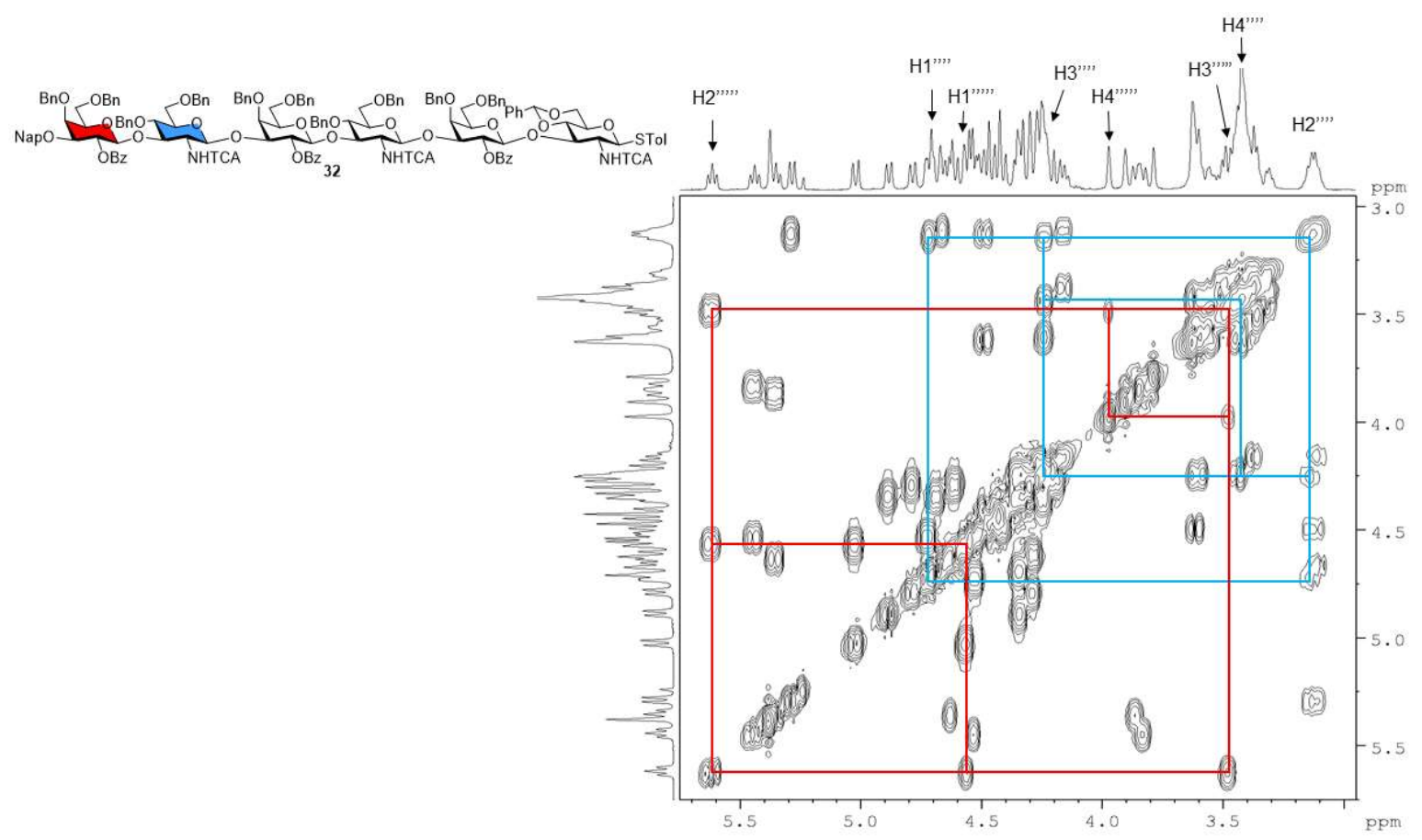

${ }^{1} \mathrm{H}-{ }^{1} \mathrm{H}$ 2D COSY NMR spectrum of compound $32\left(500 \mathrm{MHz}, \mathrm{CDCl}_{3}\right)$ 


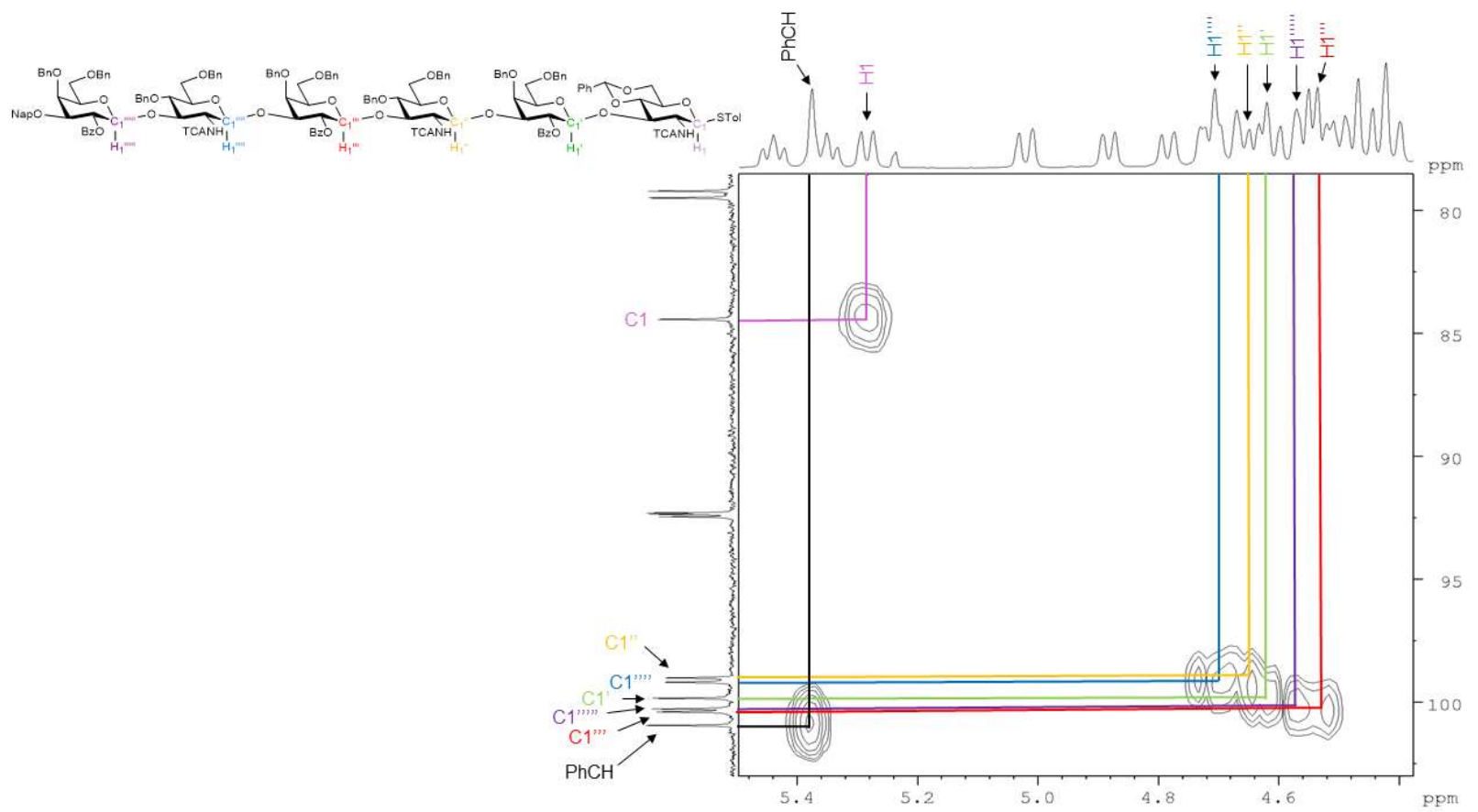

${ }^{1} \mathrm{H}-{ }^{13} \mathrm{C}$ 2D HMQC NMR spectrum of compound $32\left(500 / 125 \mathrm{MHz}, \mathrm{CDCl}_{3}\right)$ showing the selective correlations to identify the $\mathrm{C} 1$ of $\mathrm{Gal}$ and GlcNTCA residues.

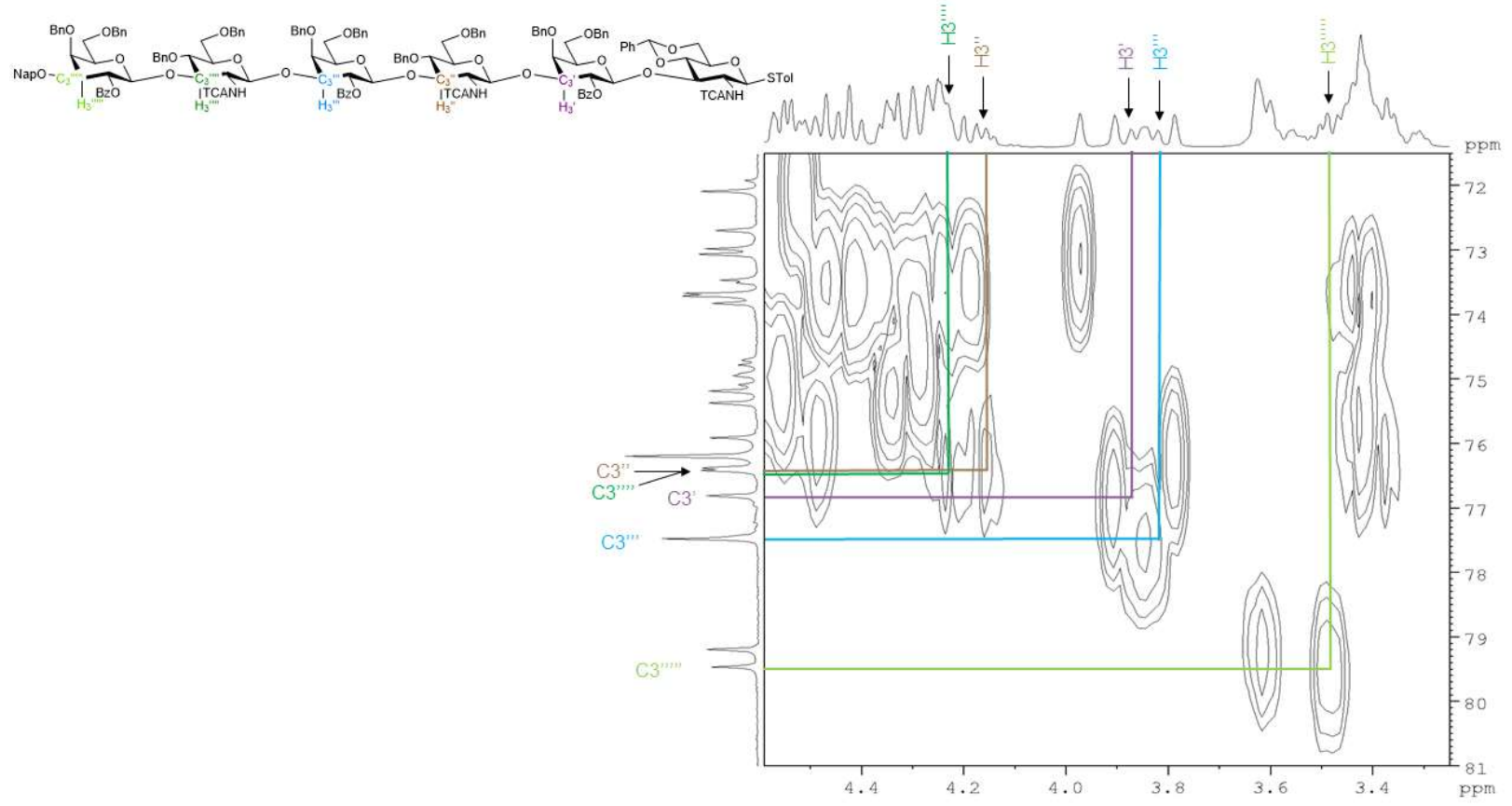

${ }^{1} \mathrm{H}-{ }^{13} \mathrm{C}$ 2D HMQC NMR spectrum of compound $32\left(500 / 125 \mathrm{MHz}, \mathrm{CDCl}_{3}\right)$ showing the selective correlations to identify the $\mathrm{C} 3$ of Gal and GlcNTCA residues. 


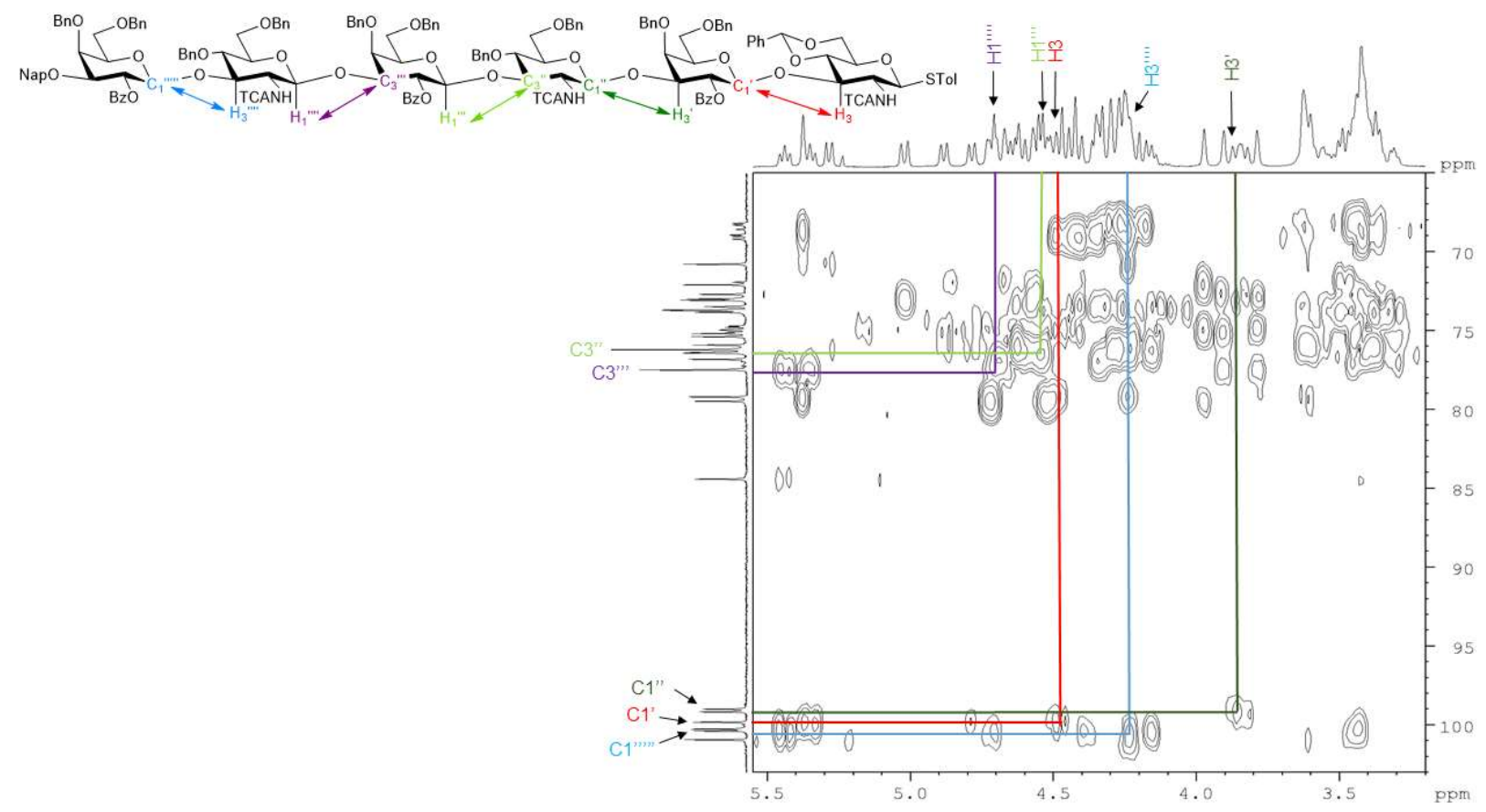

${ }^{1} \mathrm{H}-{ }^{13} \mathrm{C}$ 2D HMBC NMR spectrum of compound $32\left(500 / 125 \mathrm{MHz}, \mathrm{CDCl}_{3}\right)$ showing the selective correlations to identify the $\beta$ 1-3 linkages. 

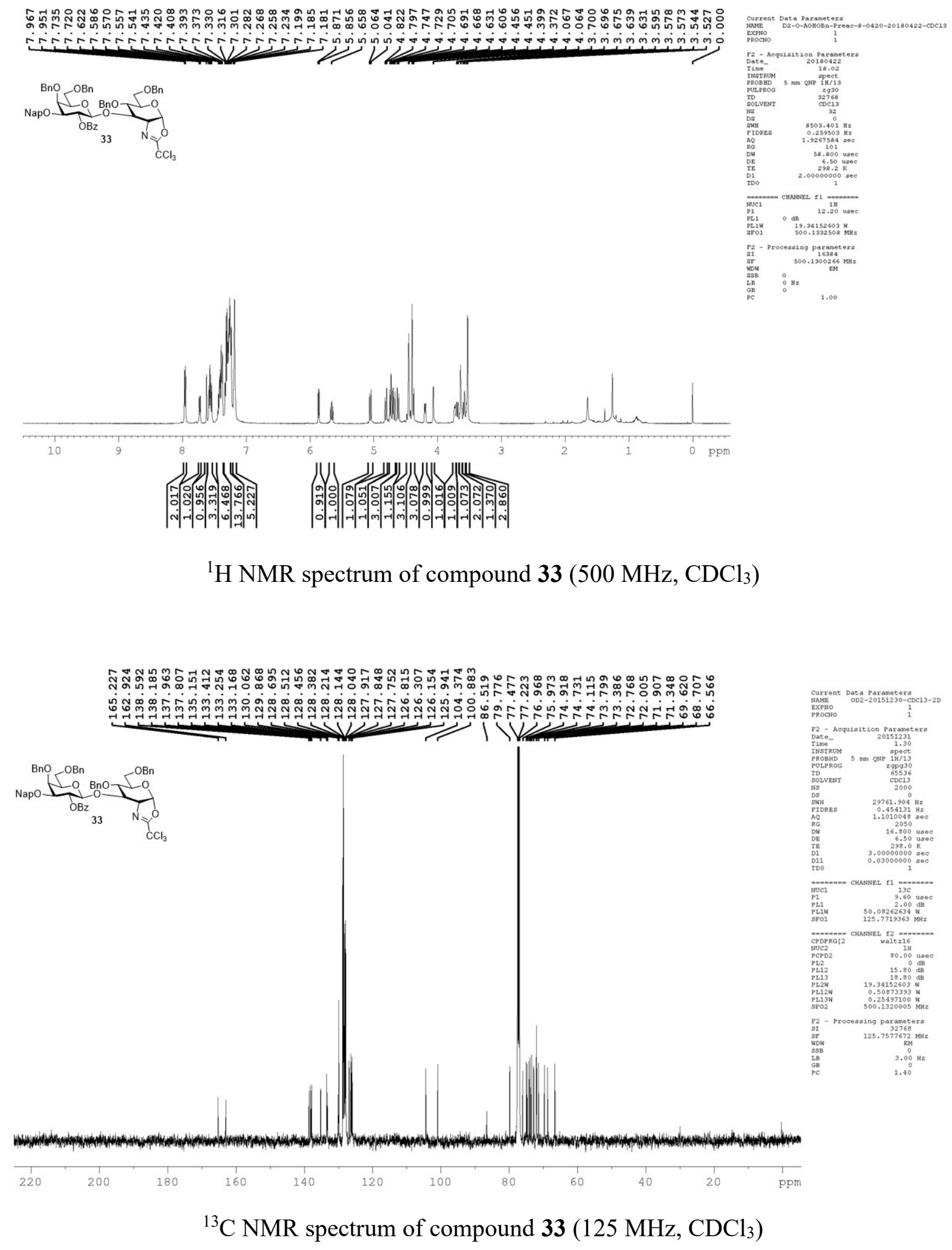


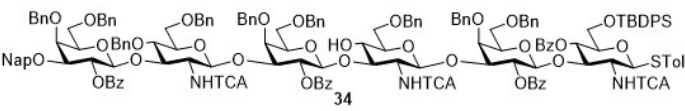

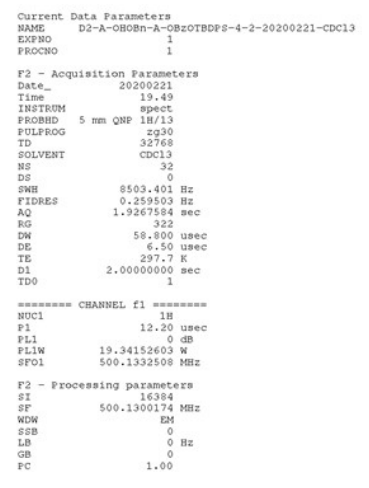

inl (N)

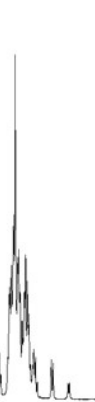

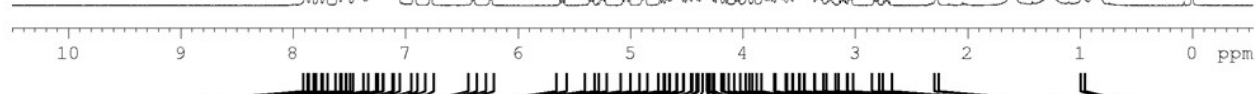

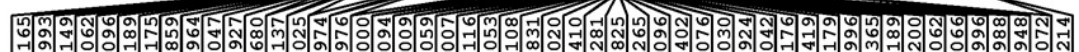

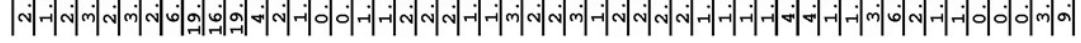

${ }^{1} \mathrm{H}$ NMR spectrum of compound $34\left(500 \mathrm{MHz}, \mathrm{CDCl}_{3}\right)$

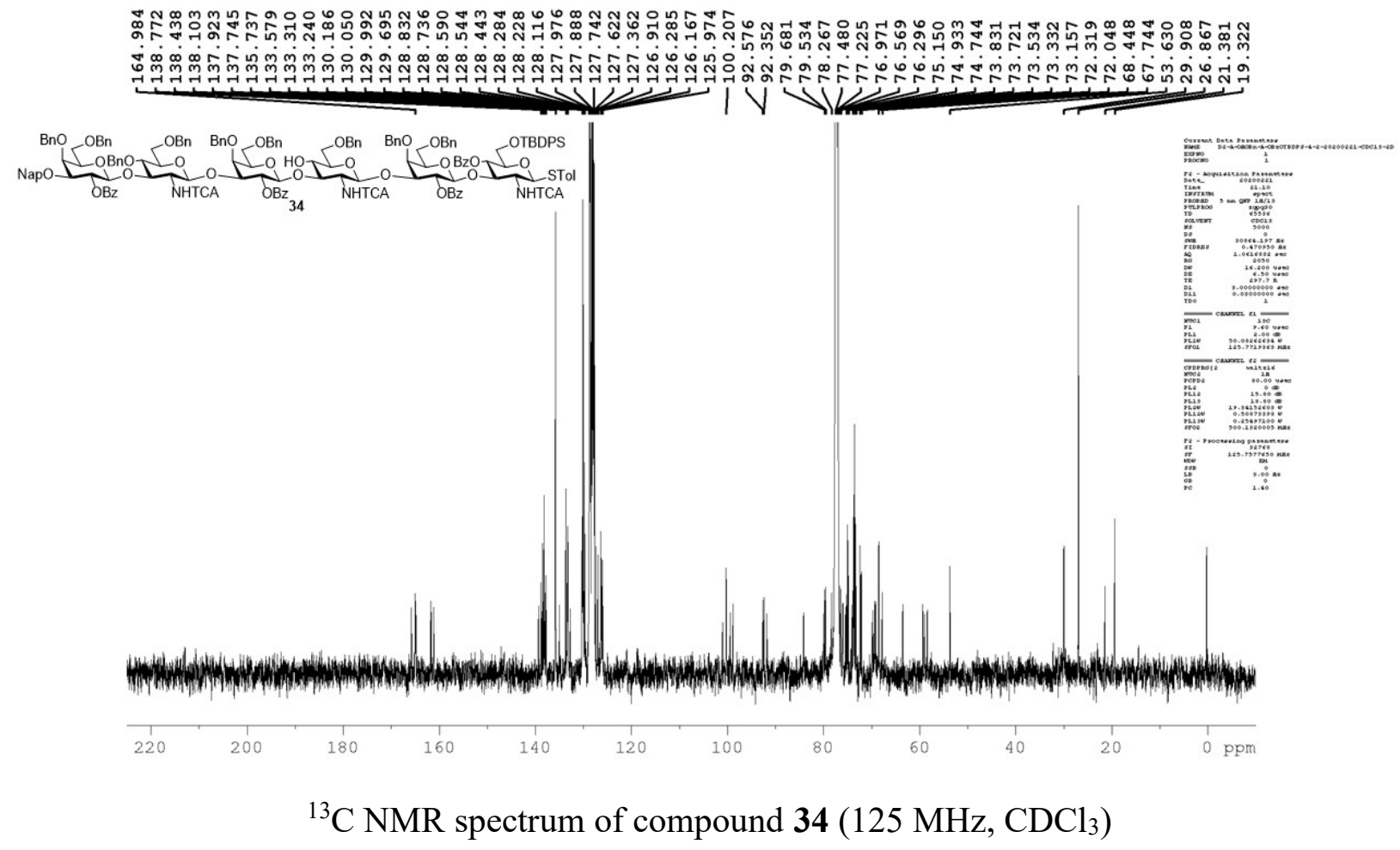



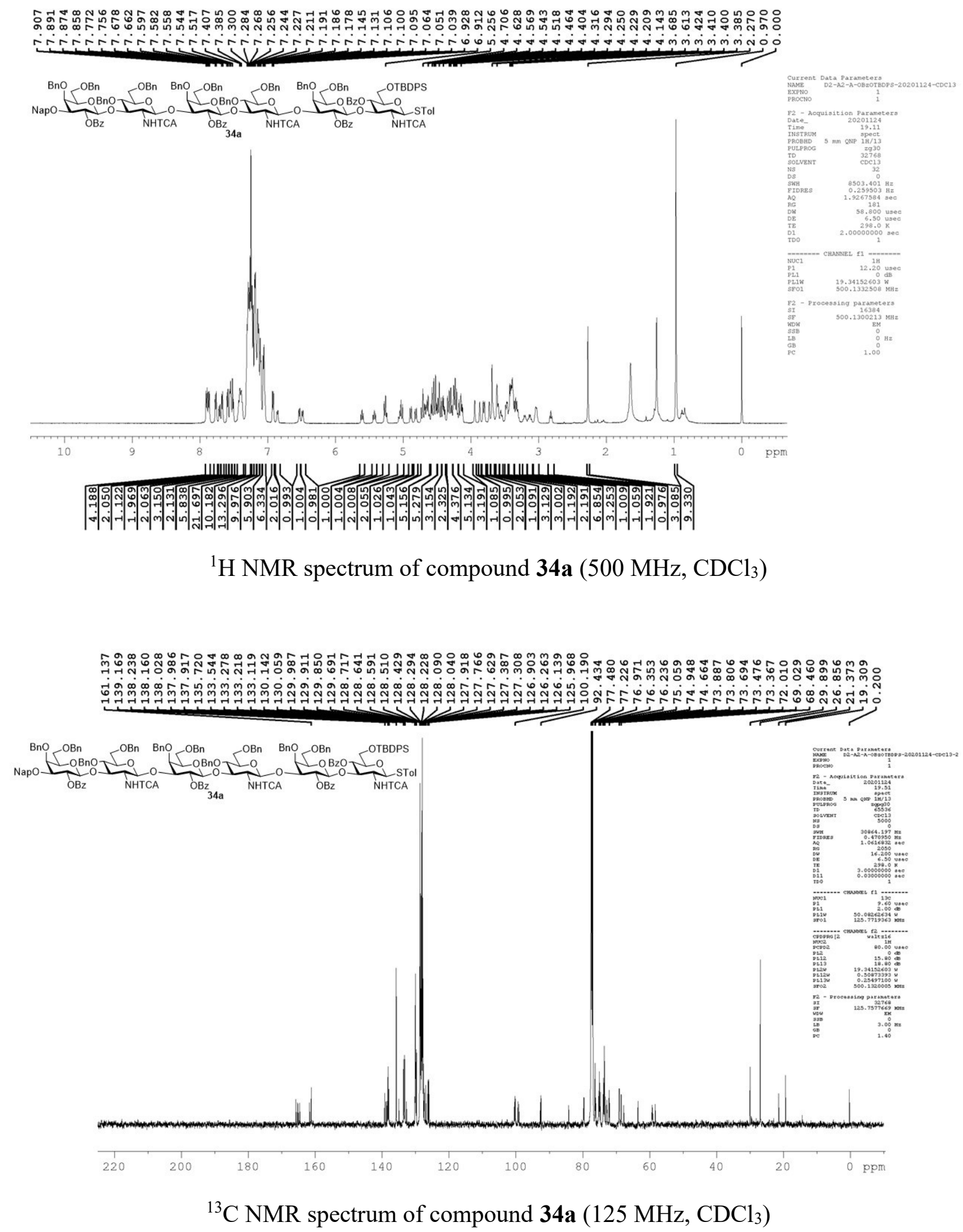

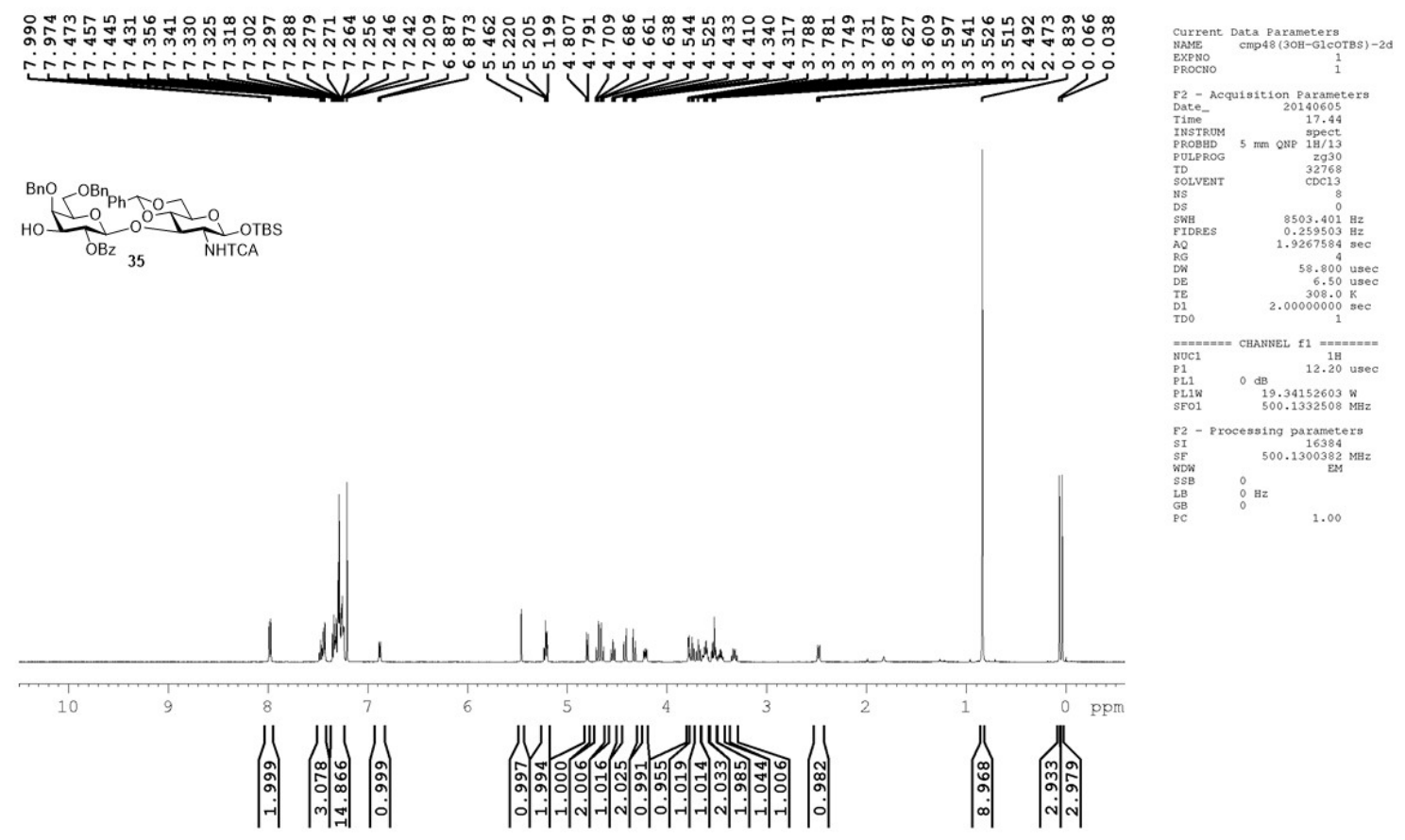

${ }^{1} \mathrm{H}$ NMR spectrum of compound $35\left(500 \mathrm{MHz}, \mathrm{CDCl}_{3}\right)$

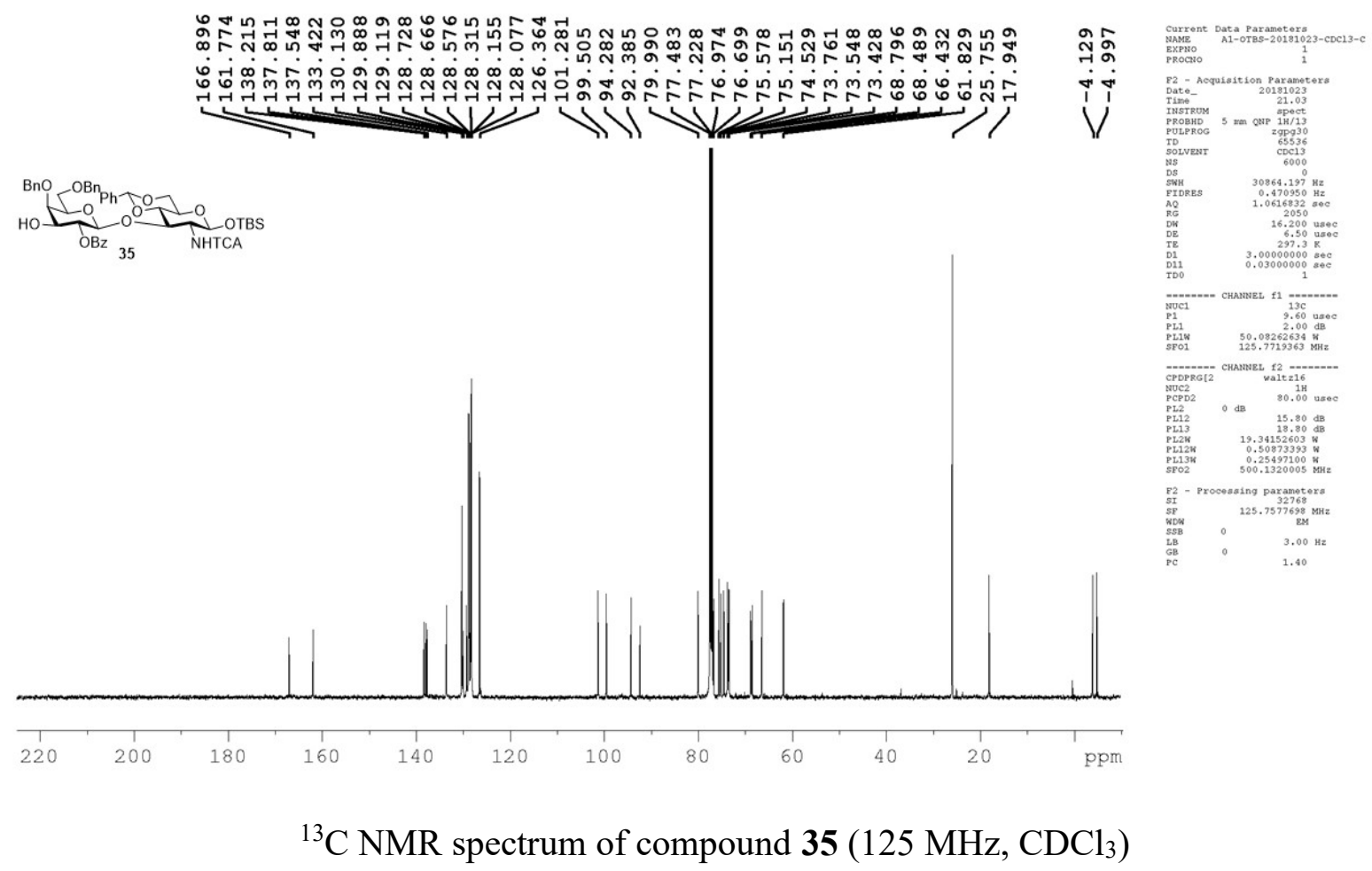




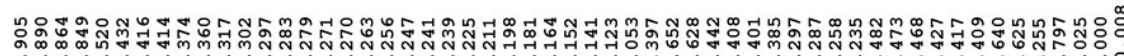

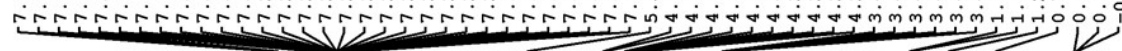

$\underbrace{\mathrm{O}}_{\mathrm{OBz}}$

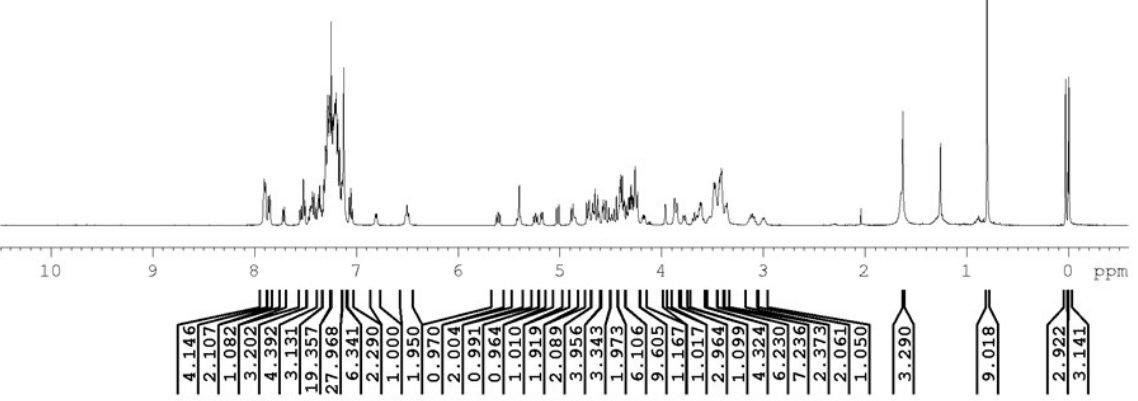

${ }^{1} \mathrm{H}$ NMR spectrum of compound $36\left(500 \mathrm{MHz}, \mathrm{CDCl}_{3}\right)$
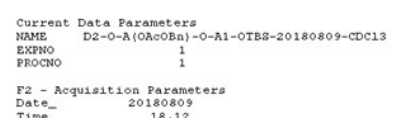

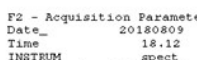

PRIRAD
PULPROOG

$\begin{array}{lr}\text { ID } & 32768 \\ \text { SOLVNT } & \text { CCC13 } \\ \text { NB } & 32 \\ \text { DS } & 0 \\ \text { SWH } & 8503.401\end{array}$

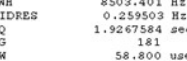

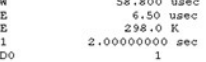

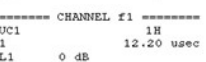

$\begin{array}{rl}19.34152603 & \mathrm{~W} \\ \mathrm{rol} & 500.1332508 \mathrm{MH}\end{array}$

F2- Processing parameters

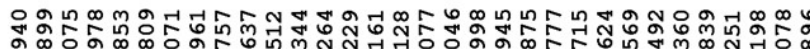

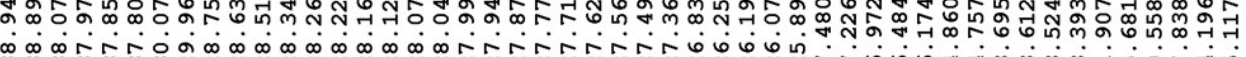

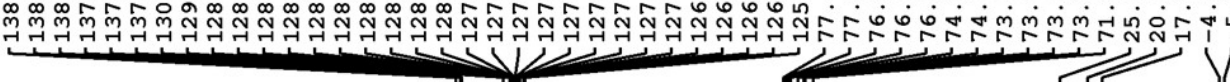

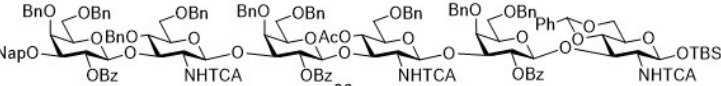
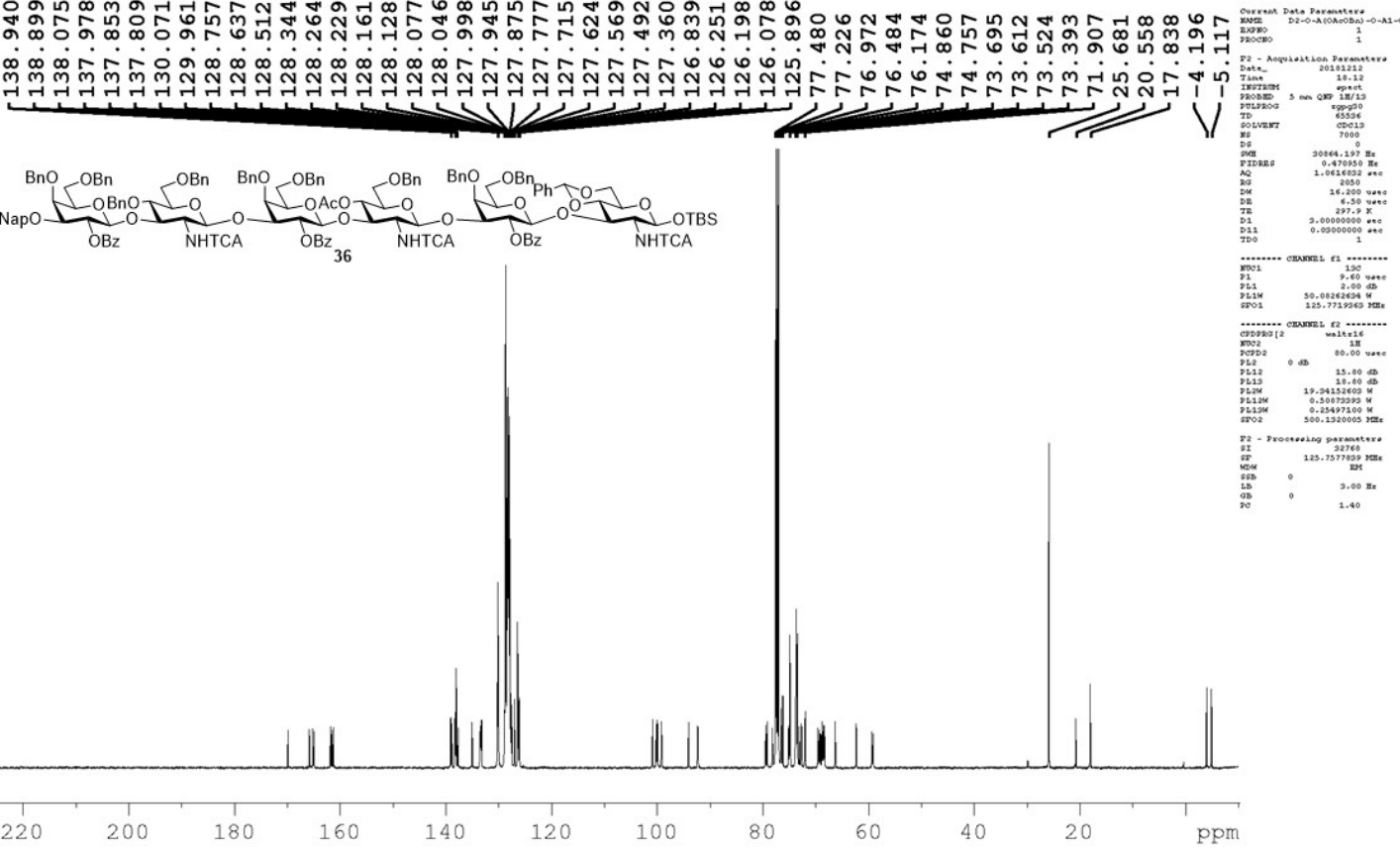

20

ppm

${ }^{13} \mathrm{C}$ NMR spectrum of compound $\mathbf{3 6}\left(125 \mathrm{MHz}, \mathrm{CDCl}_{3}\right)$ 


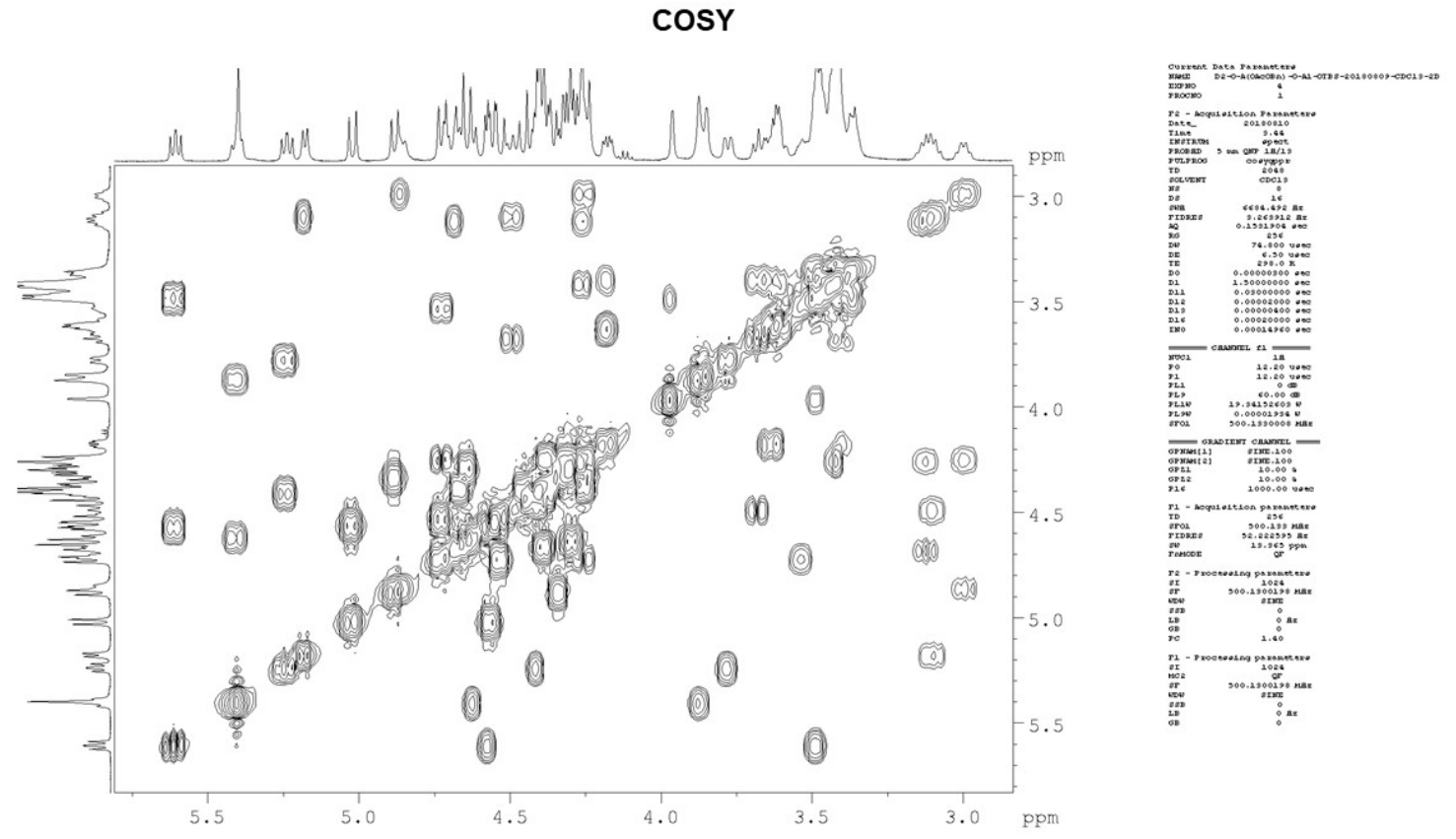

${ }^{1} \mathrm{H}-{ }^{1} \mathrm{H}$ 2D COSY NMR spectrum of compound $36\left(500 \mathrm{MHz}, \mathrm{CDCl}_{3}\right)$
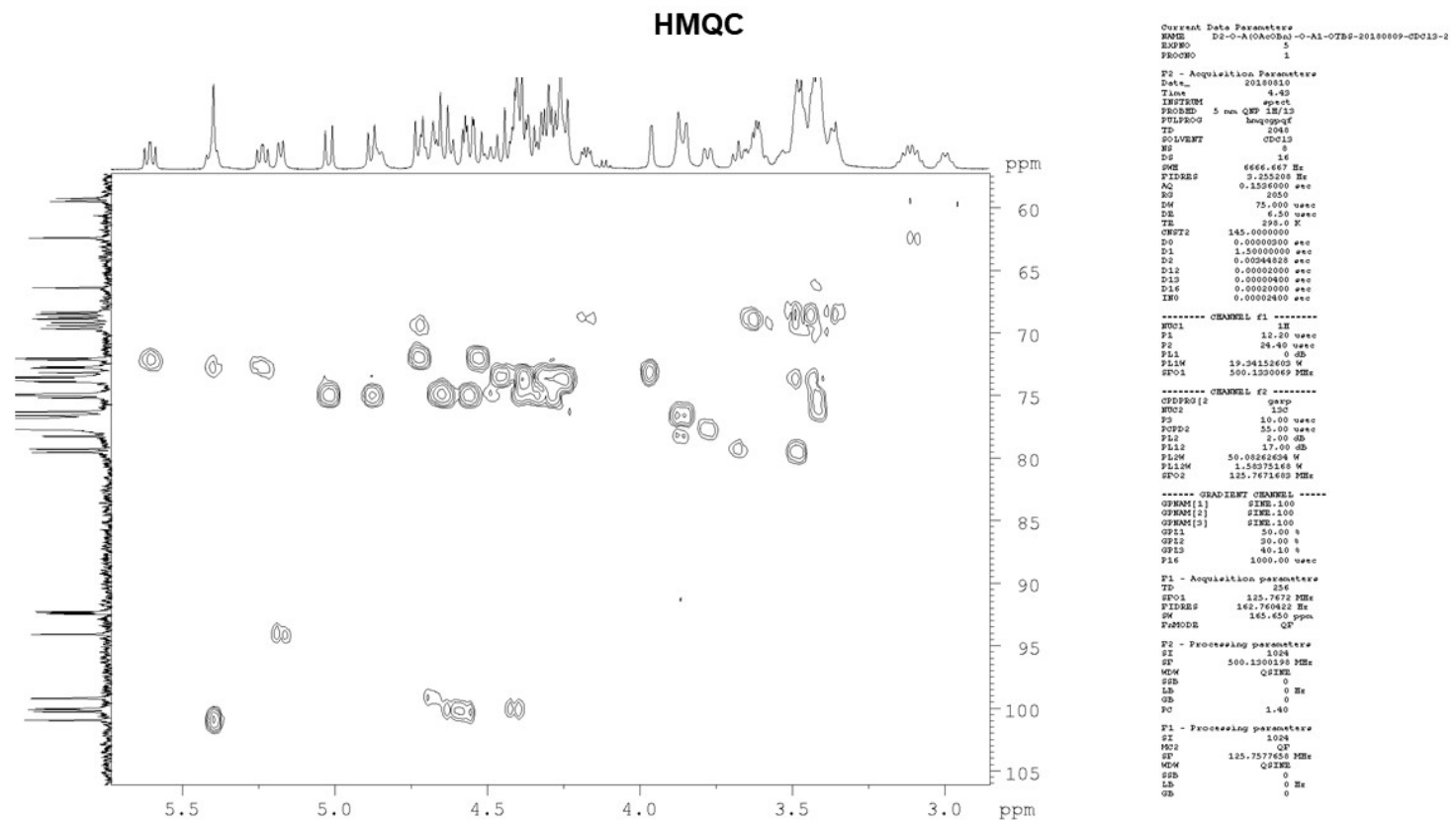

${ }^{1} \mathrm{H}_{-}{ }^{13} \mathrm{C}$ 2D HMQC NMR spectrum of compound $36\left(500 / 125 \mathrm{MHz}, \mathrm{CDCl}_{3}\right)$ 

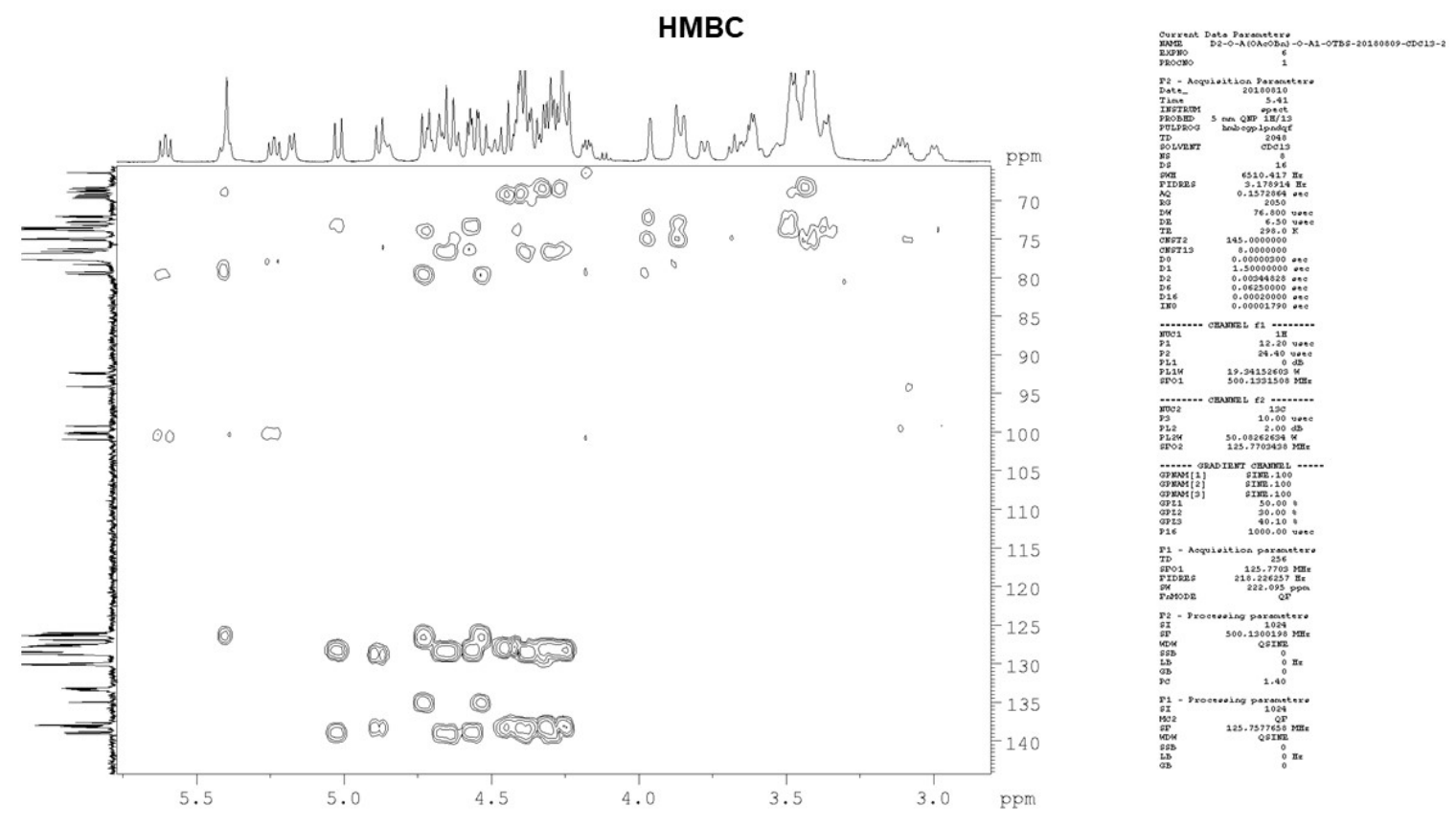

${ }^{1} \mathrm{H}_{-}{ }^{13} \mathrm{C}$ 2D HMBC NMR spectrum of compound $36\left(500 / 125 \mathrm{MHz}, \mathrm{CDCl}_{3}\right)$ 


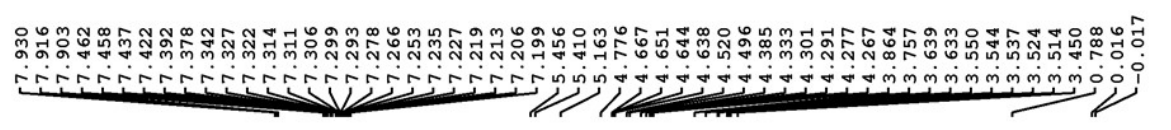

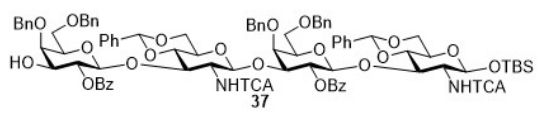
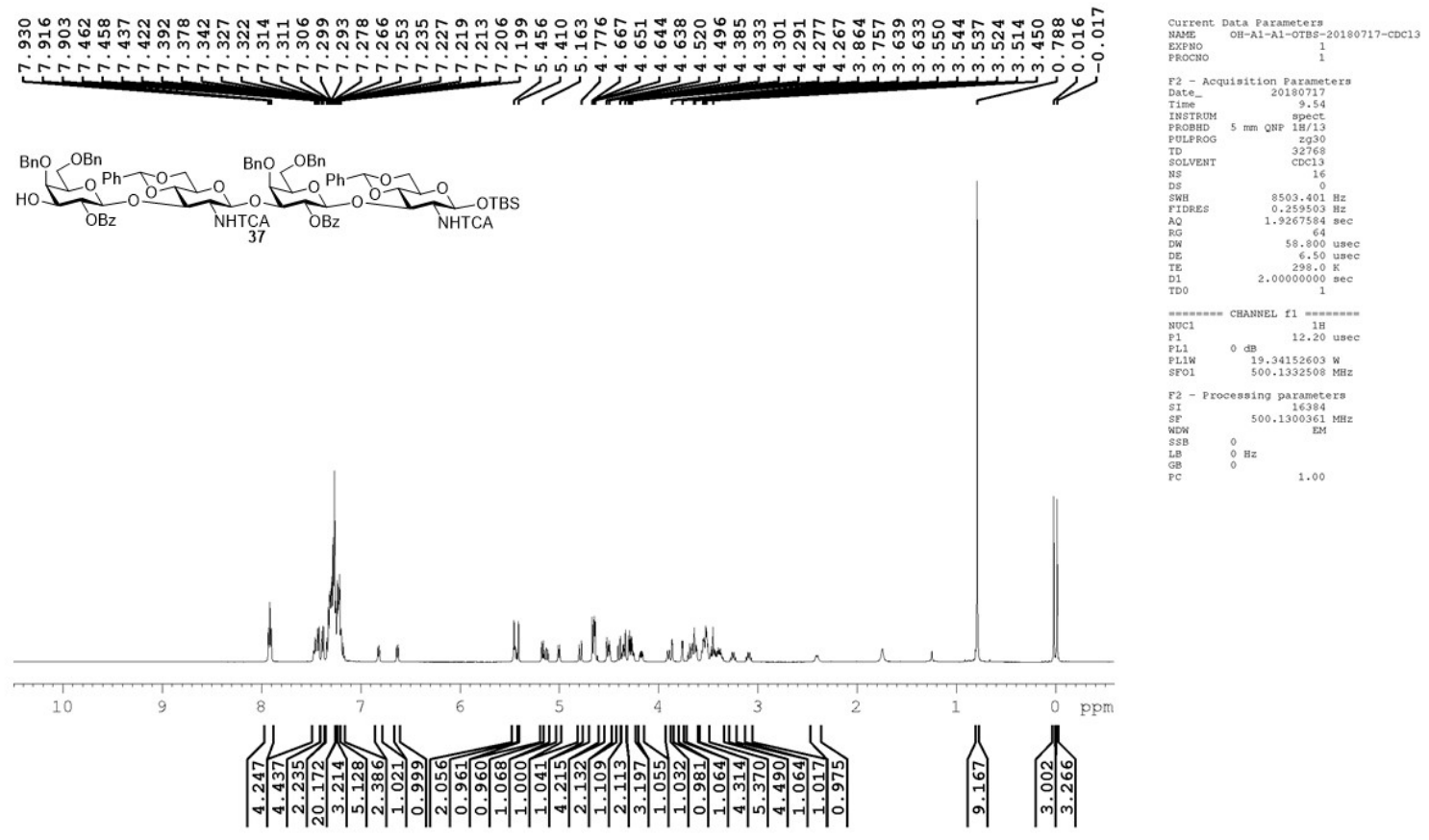

${ }^{1} \mathrm{H}$ NMR spectrum of compound $37\left(500 \mathrm{MHz}, \mathrm{CDCl}_{3}\right)$

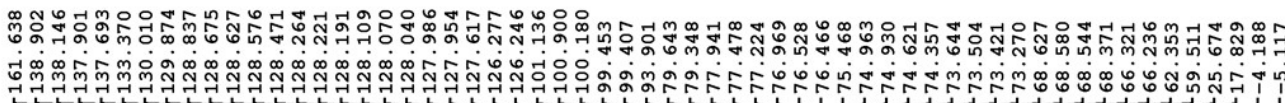
பొ

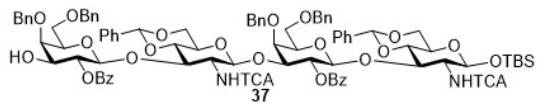
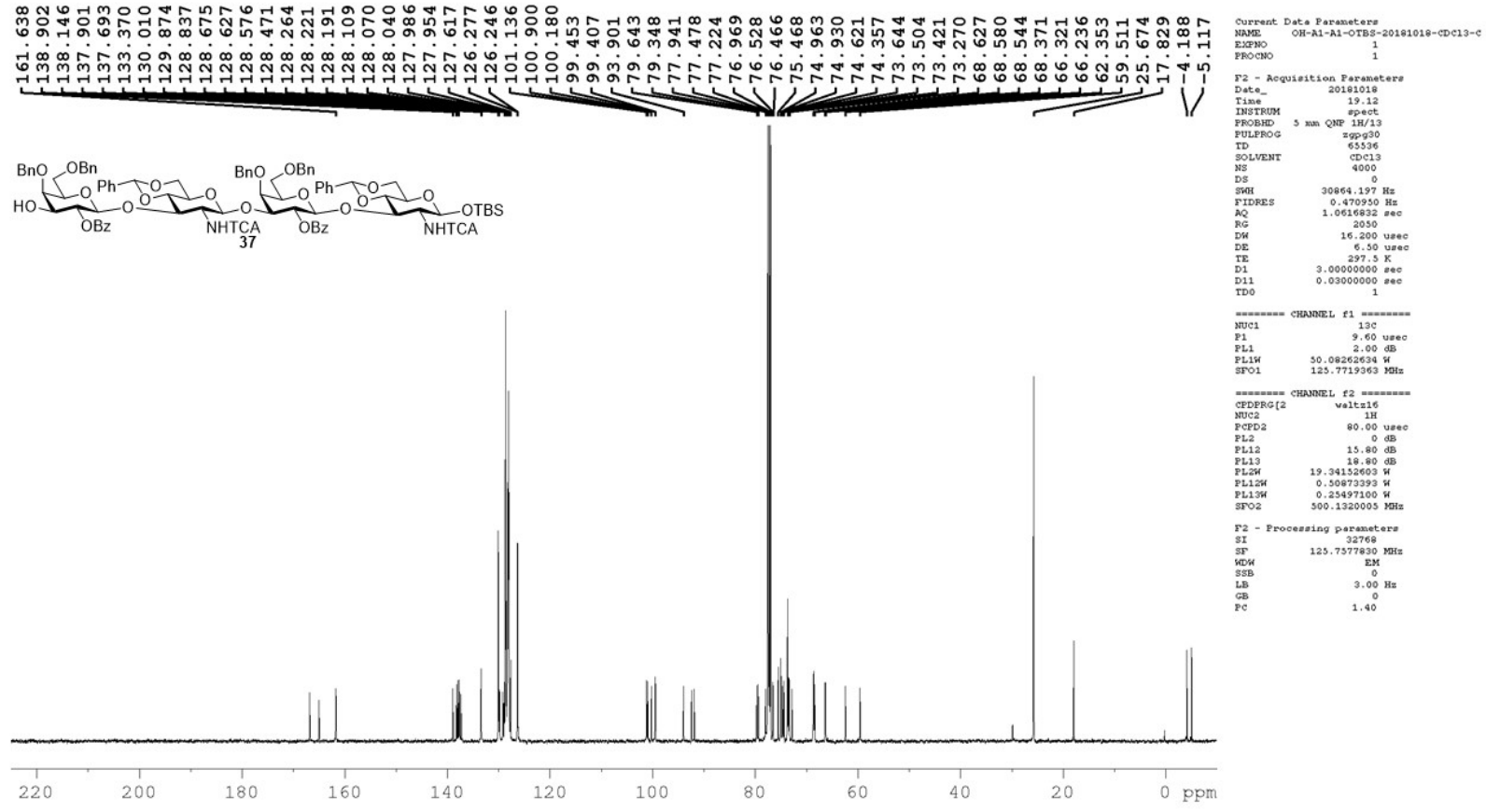

${ }^{13} \mathrm{C}$ NMR spectrum of compound $37\left(125 \mathrm{MHz}, \mathrm{CDCl}_{3}\right)$ 


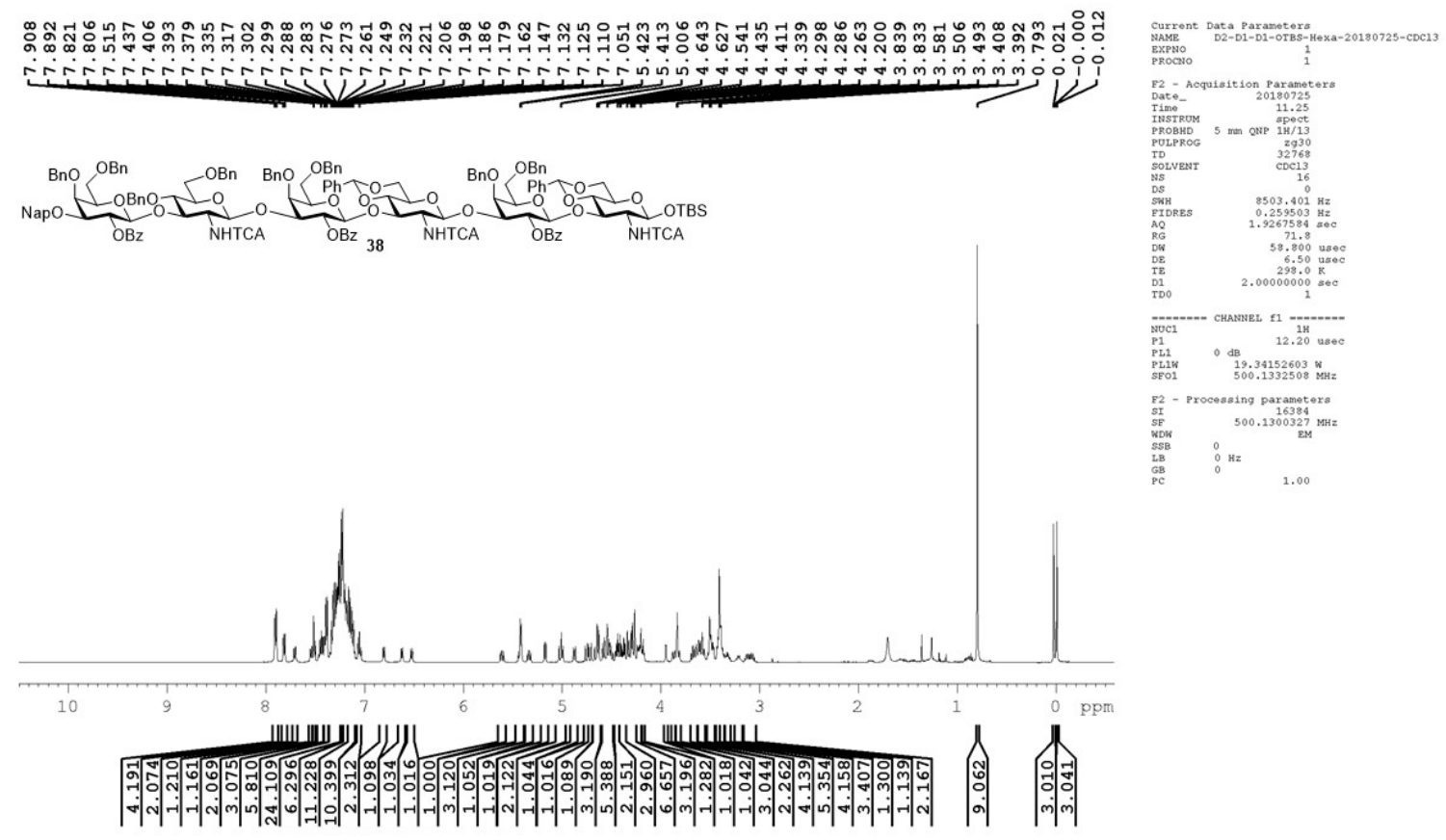

${ }^{1} \mathrm{H}$ NMR spectrum of compound $38\left(500 \mathrm{MHz}, \mathrm{CDCl}_{3}\right)$

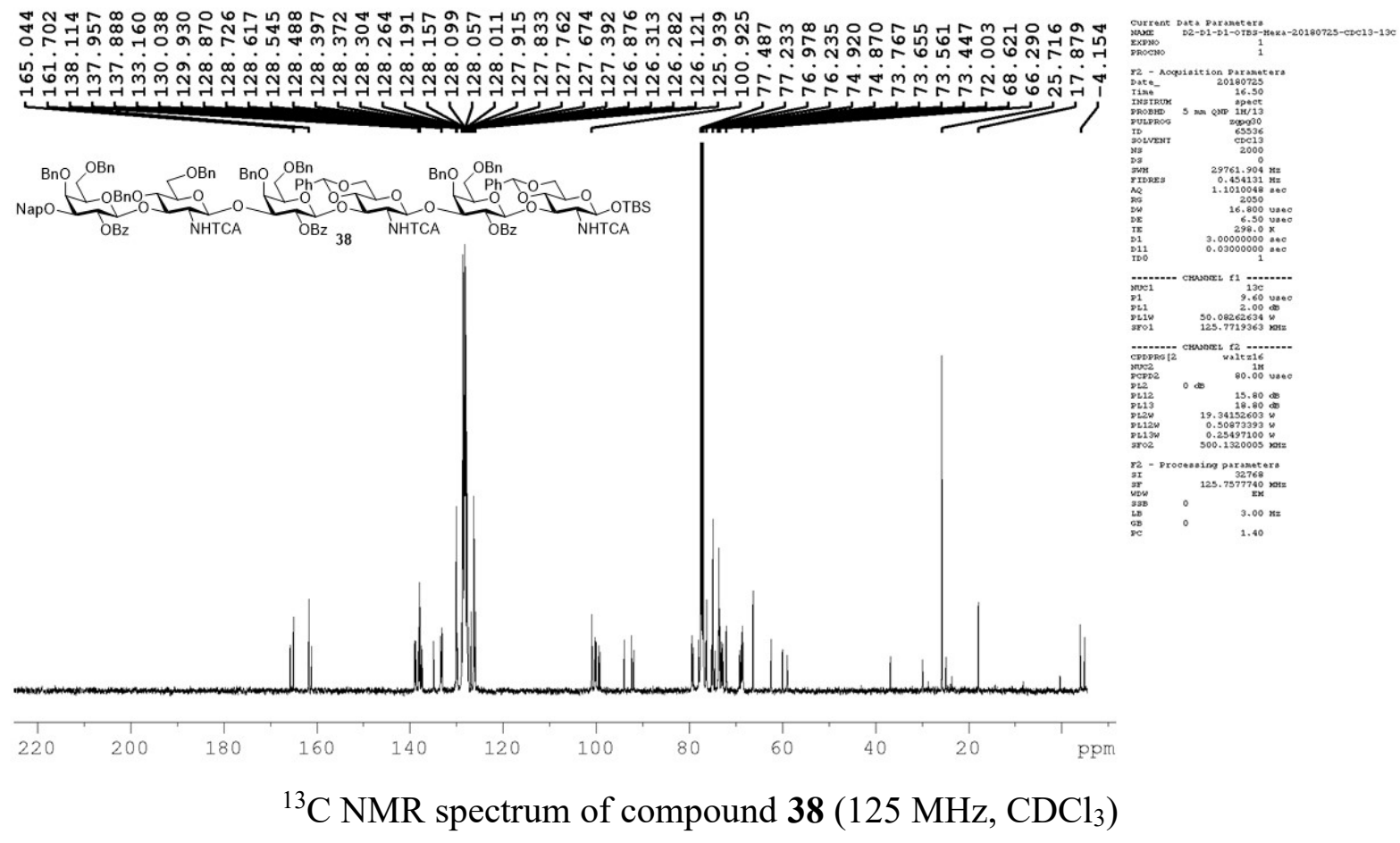



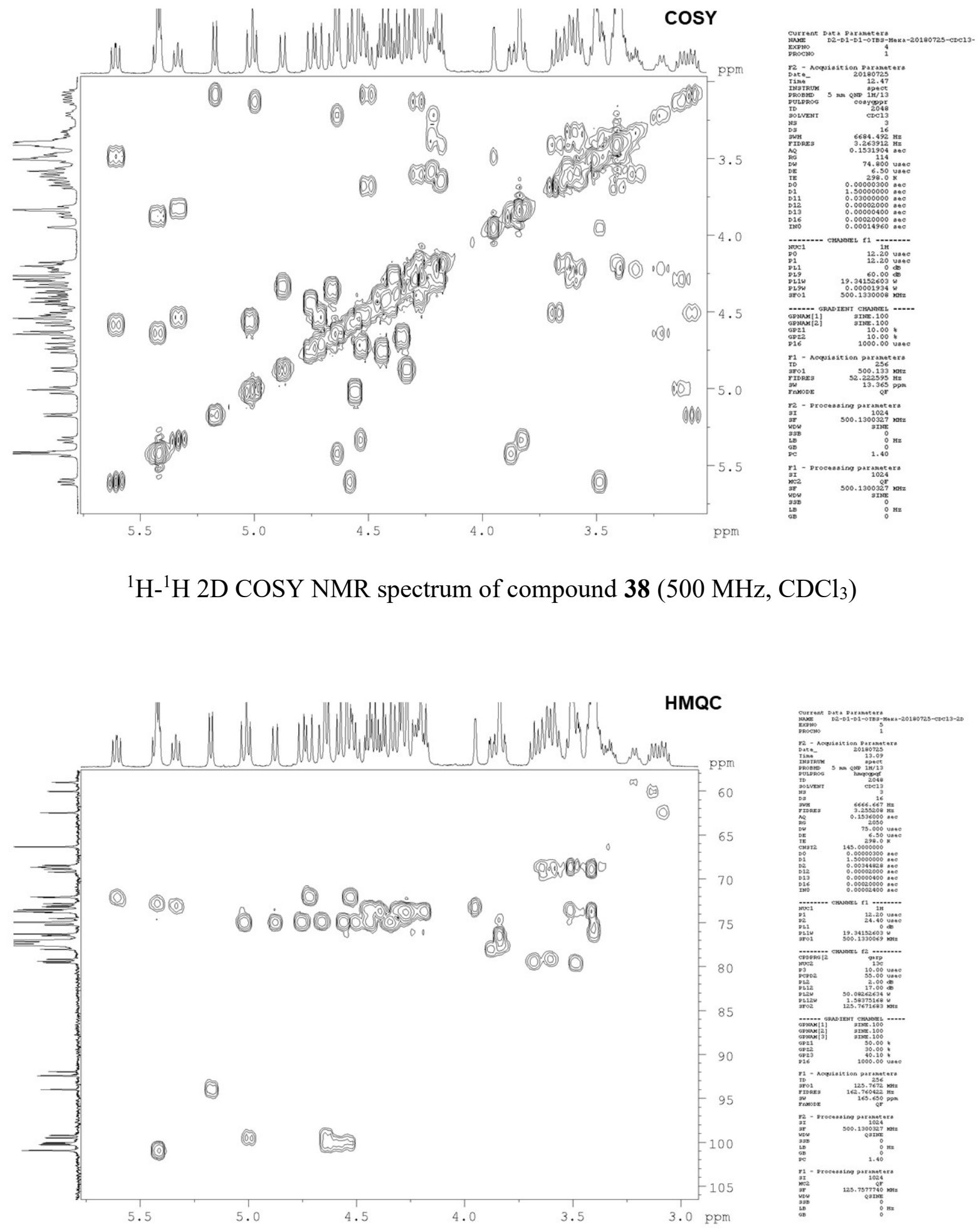

${ }^{1} \mathrm{H}_{-}{ }^{13} \mathrm{C} 2 \mathrm{D}$ HMQC NMR spectrum of compound $38\left(500 / 125 \mathrm{MHz}, \mathrm{CDCl}_{3}\right)$ 

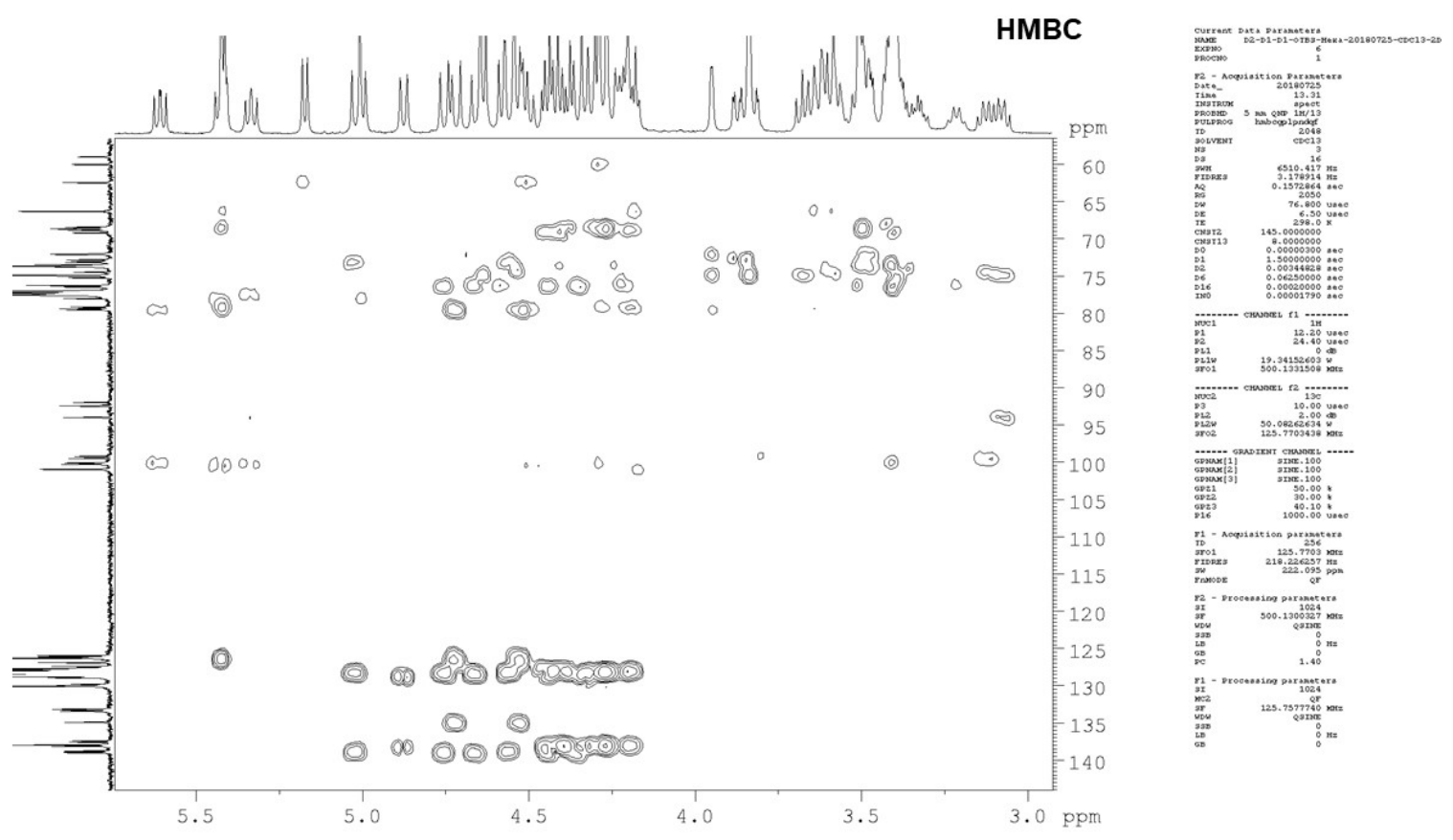

${ }^{1} \mathrm{H}-{ }^{13} \mathrm{C} 2 \mathrm{D}$ HMBC NMR spectrum of compound $38\left(500 / 125 \mathrm{MHz}, \mathrm{CDCl}_{3}\right)$ 


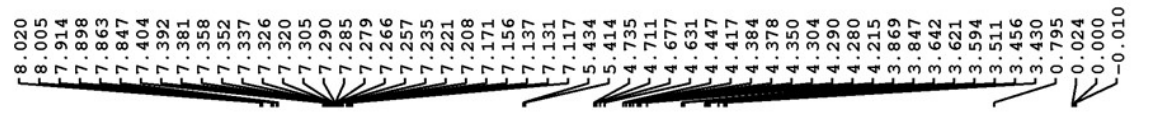

$\underbrace{\mathrm{OBn}}_{\mathrm{OBz}} \underbrace{\mathrm{OBn}}_{\mathrm{NHTCA}}$

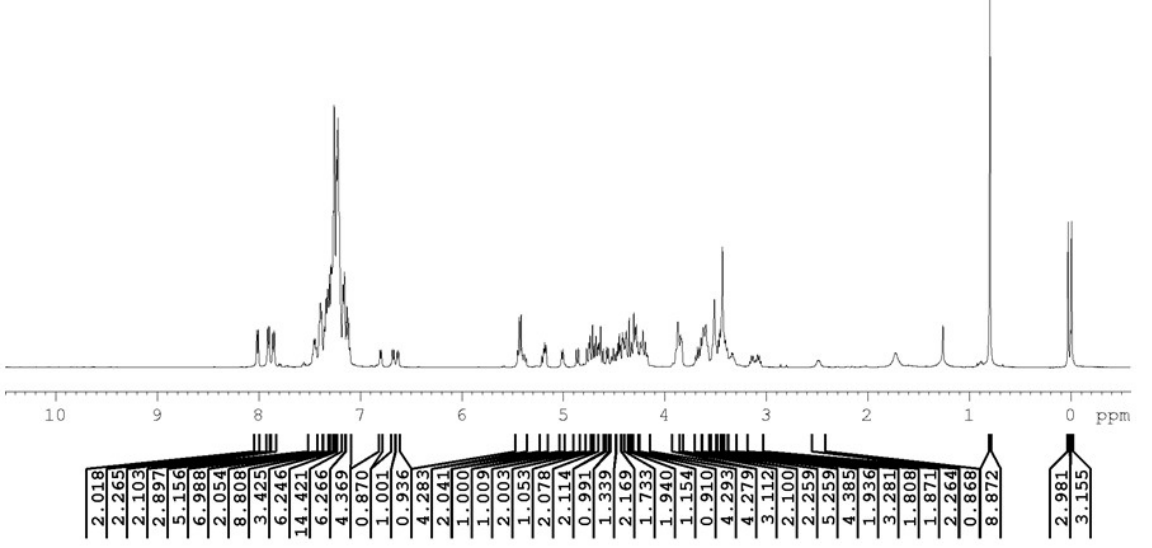

${ }^{1} \mathrm{H}$ NMR spectrum of compound $39\left(500 \mathrm{MHz}, \mathrm{CDCl}_{3}\right)$
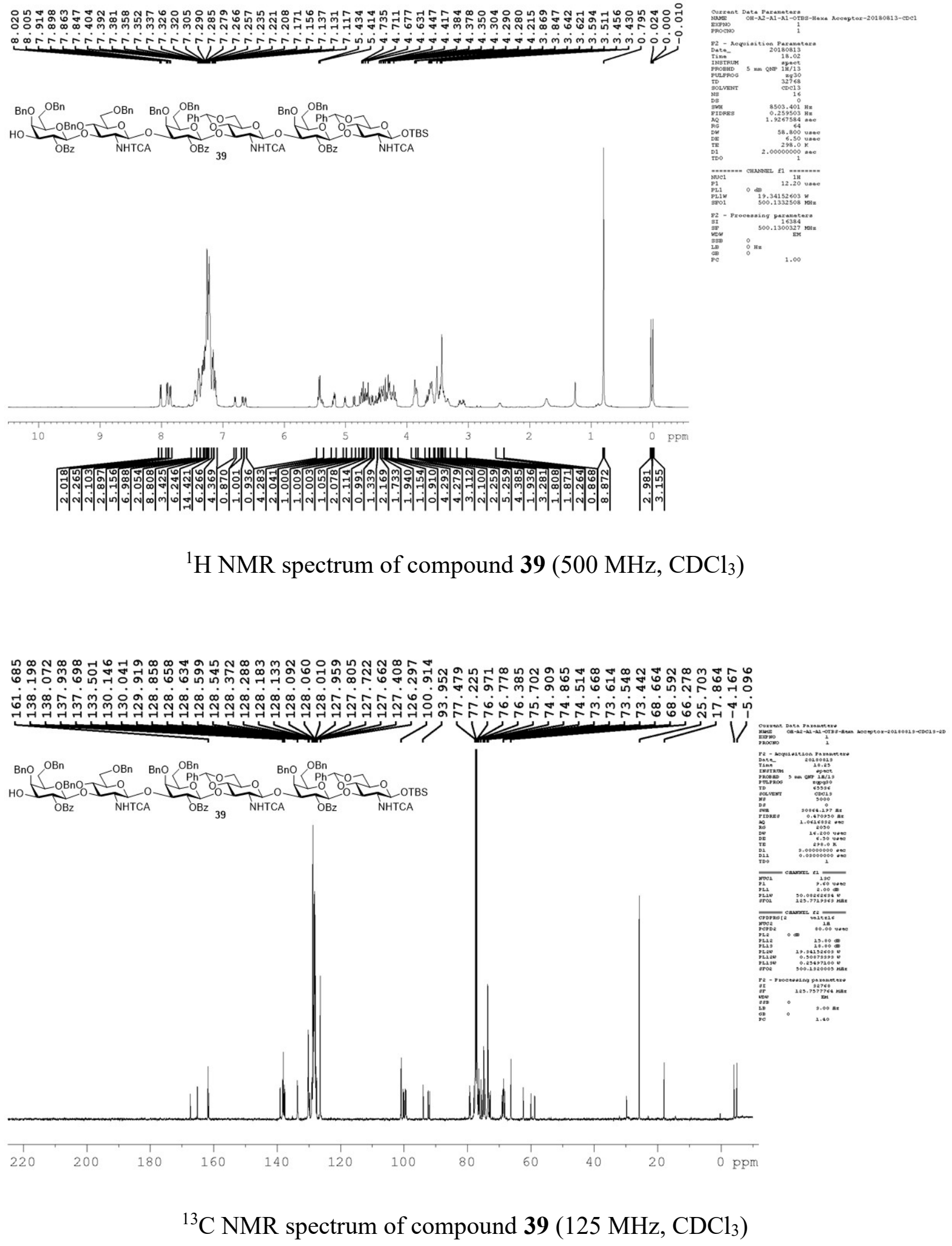


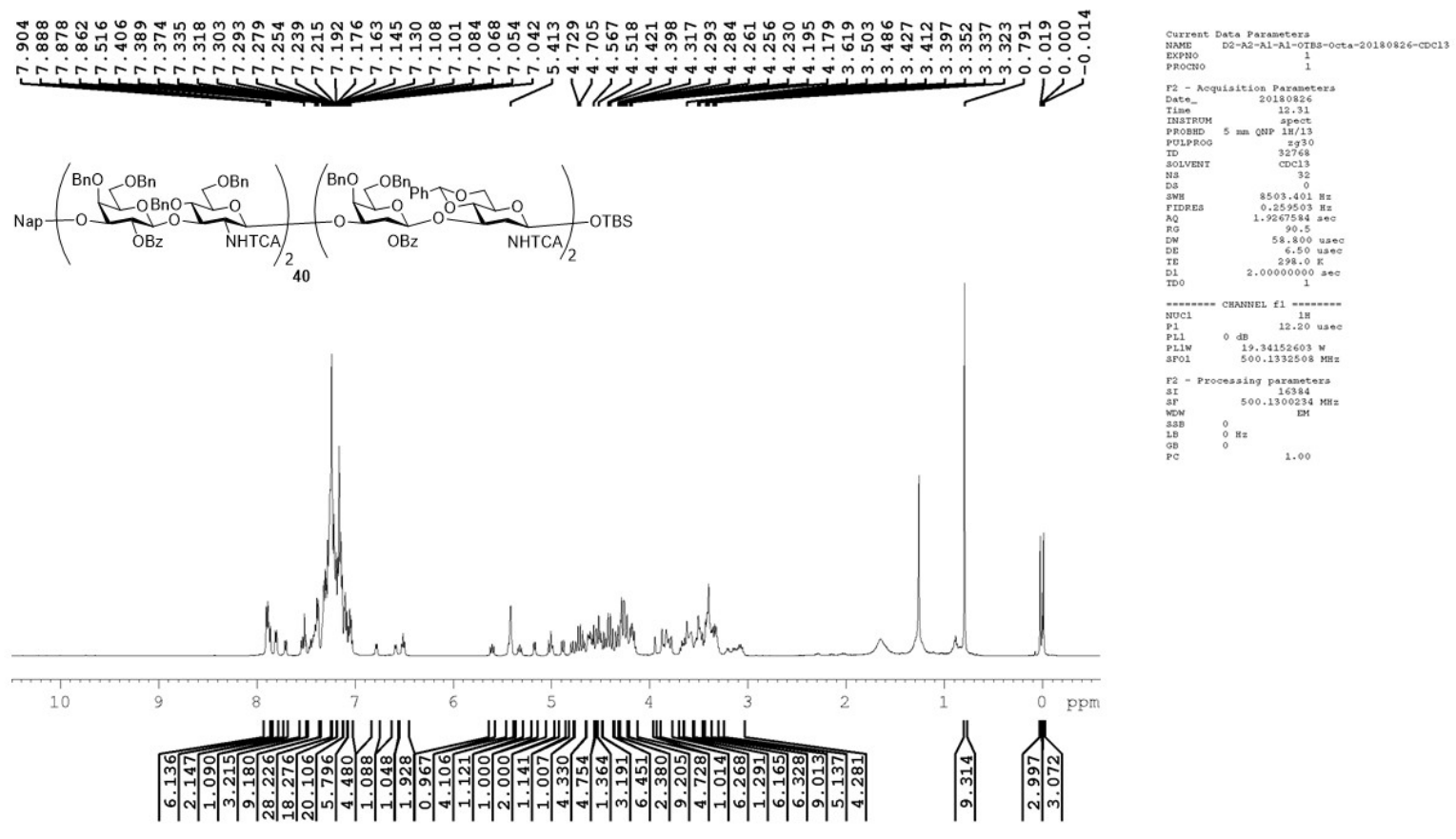

${ }^{1} \mathrm{H}$ NMR spectrum of compound $40\left(500 \mathrm{MHz}, \mathrm{CDCl}_{3}\right)$

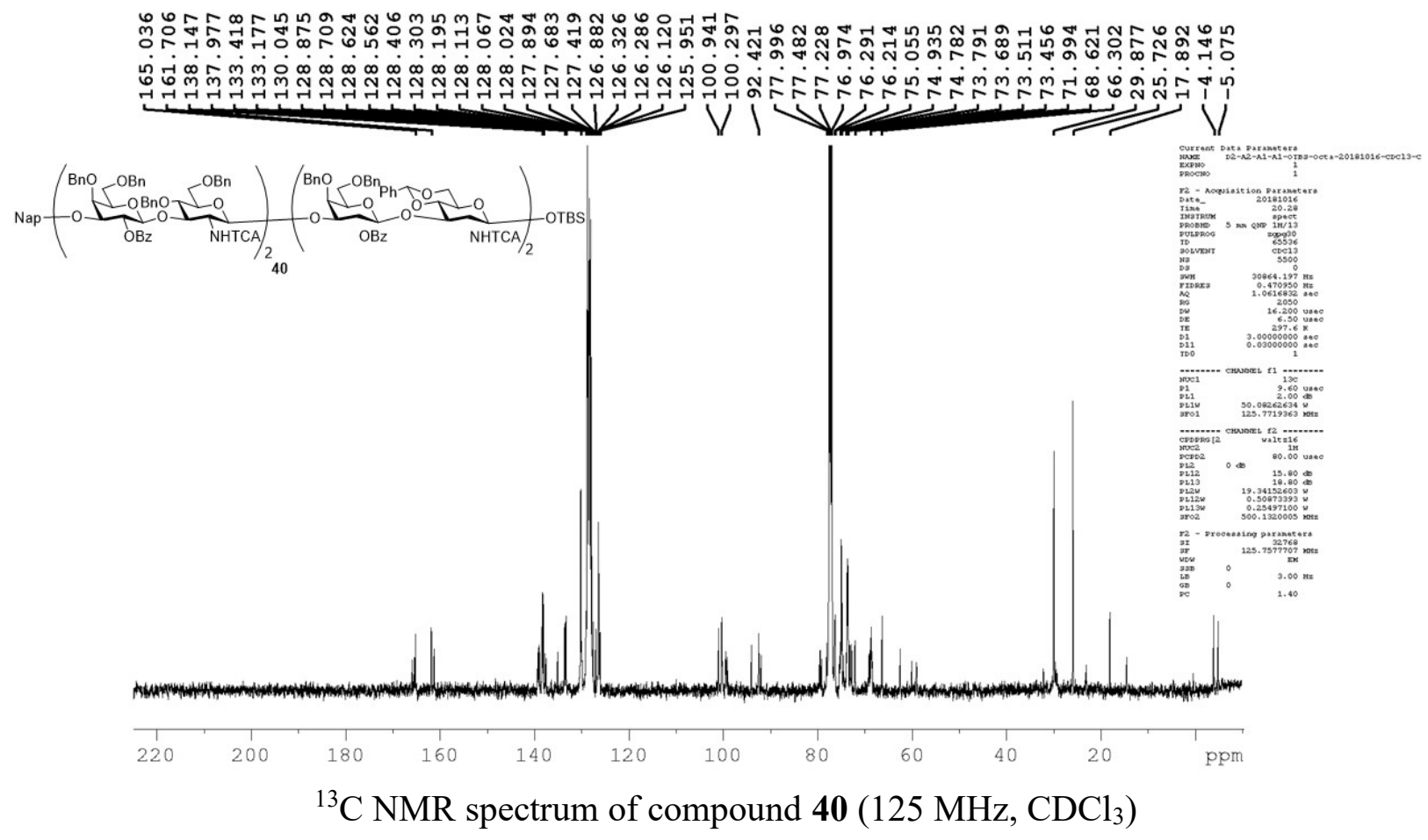



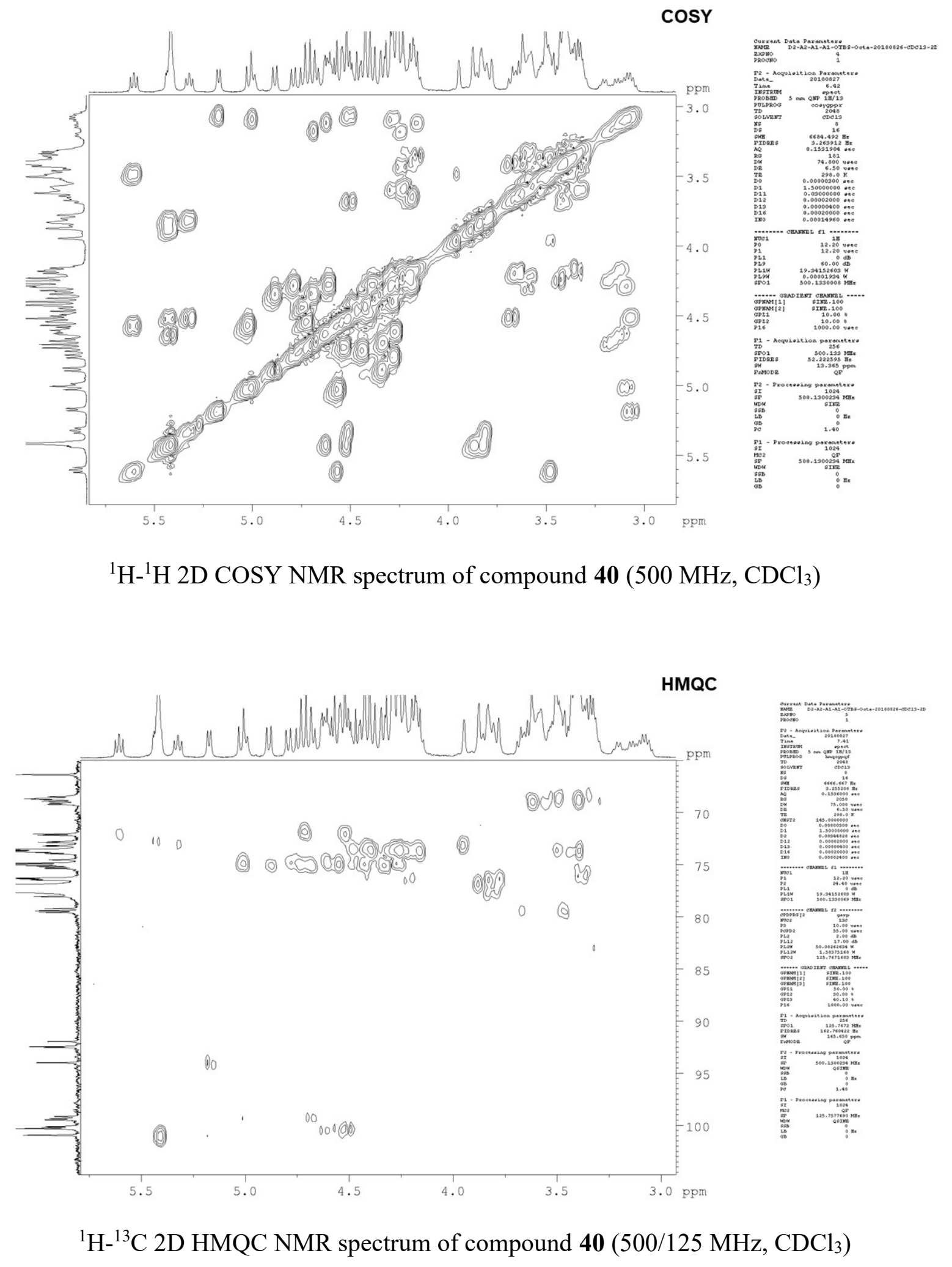

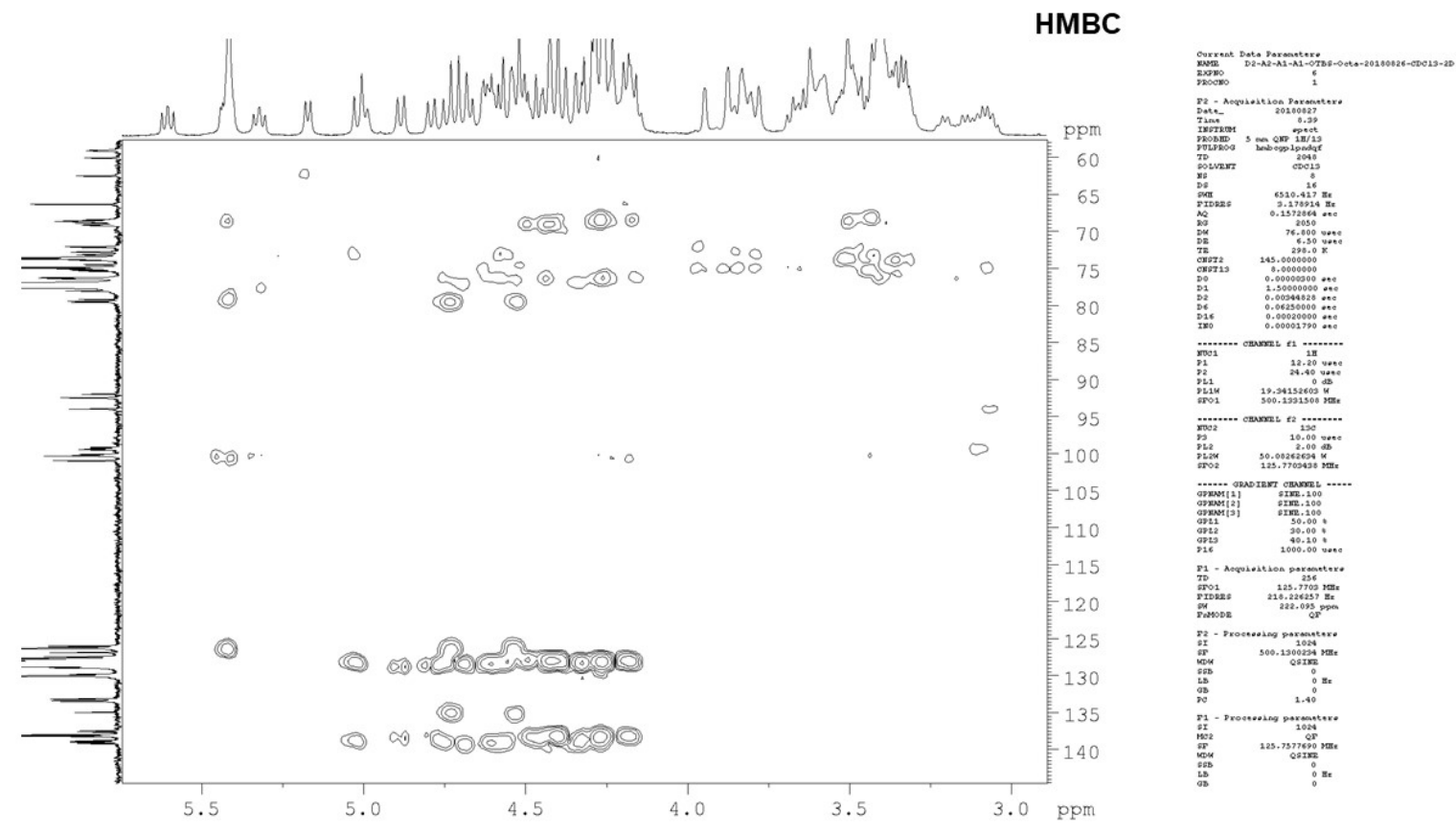

${ }^{1} \mathrm{H}-{ }^{13} \mathrm{C} 2 \mathrm{D}$ HMBC NMR spectrum of compound $40\left(500 / 125 \mathrm{MHz}, \mathrm{CDCl}_{3}\right)$ 


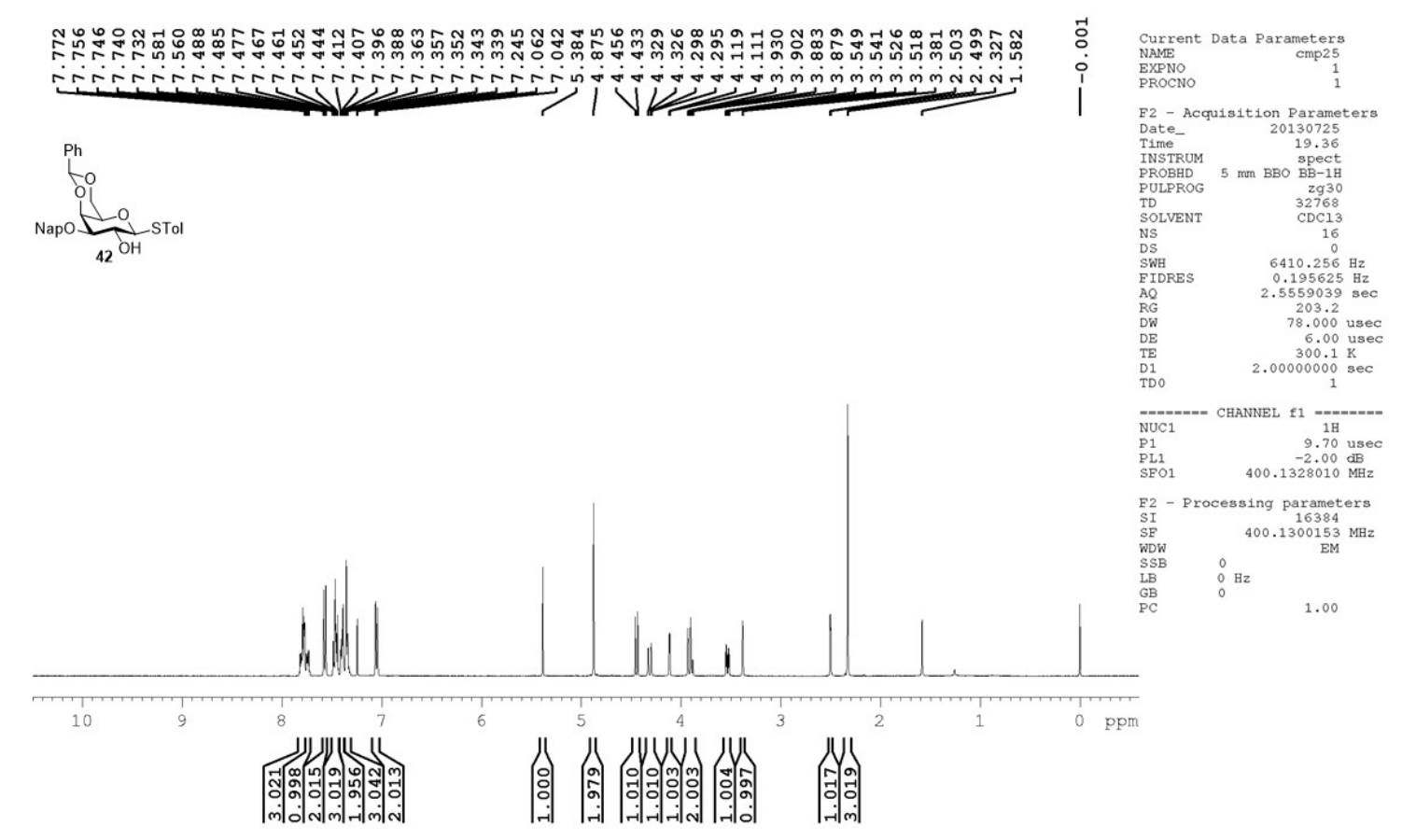

${ }^{1} \mathrm{H}$ NMR spectrum of compound $42\left(400 \mathrm{MHz}, \mathrm{CDCl}_{3}\right)$

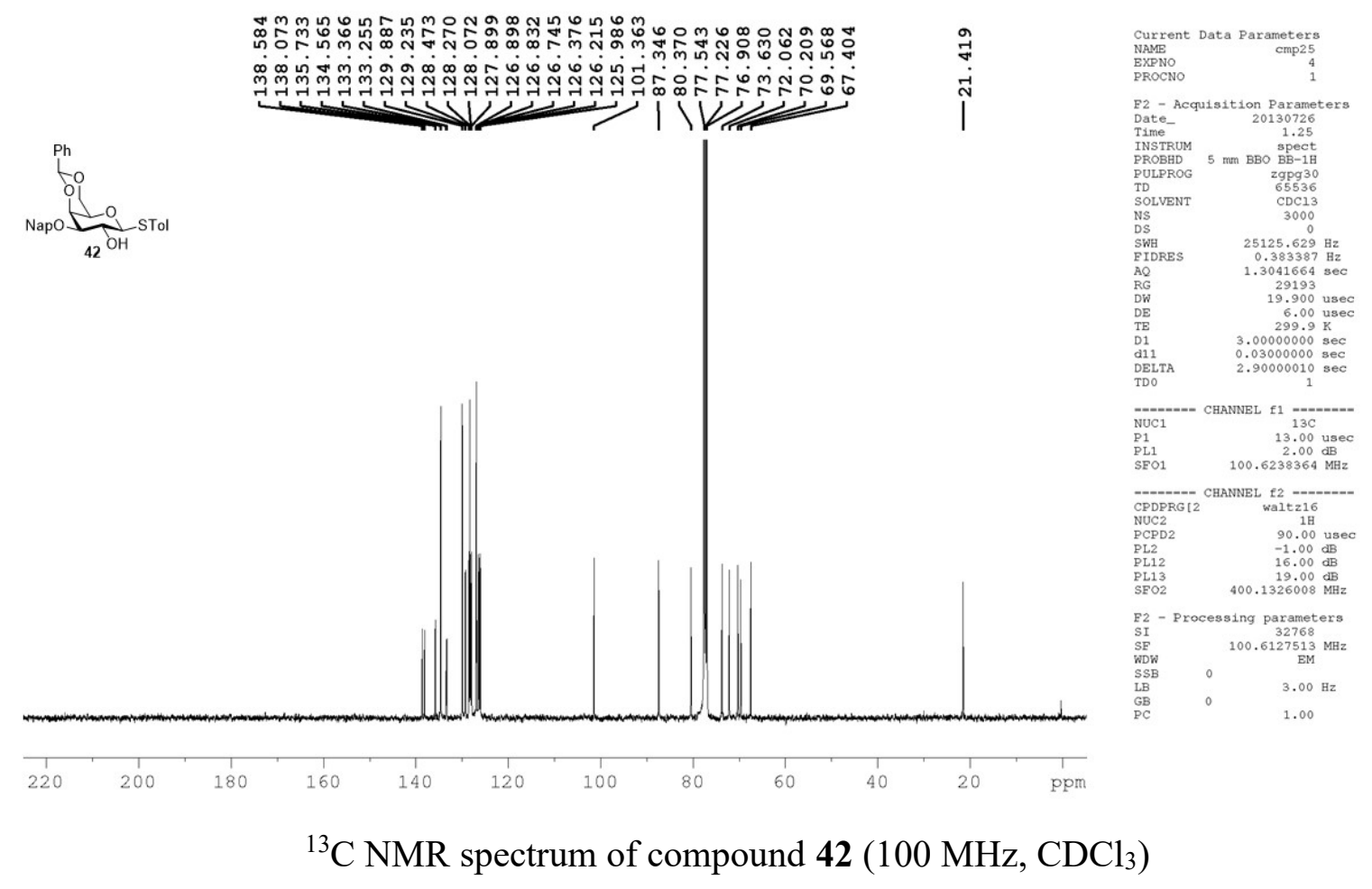



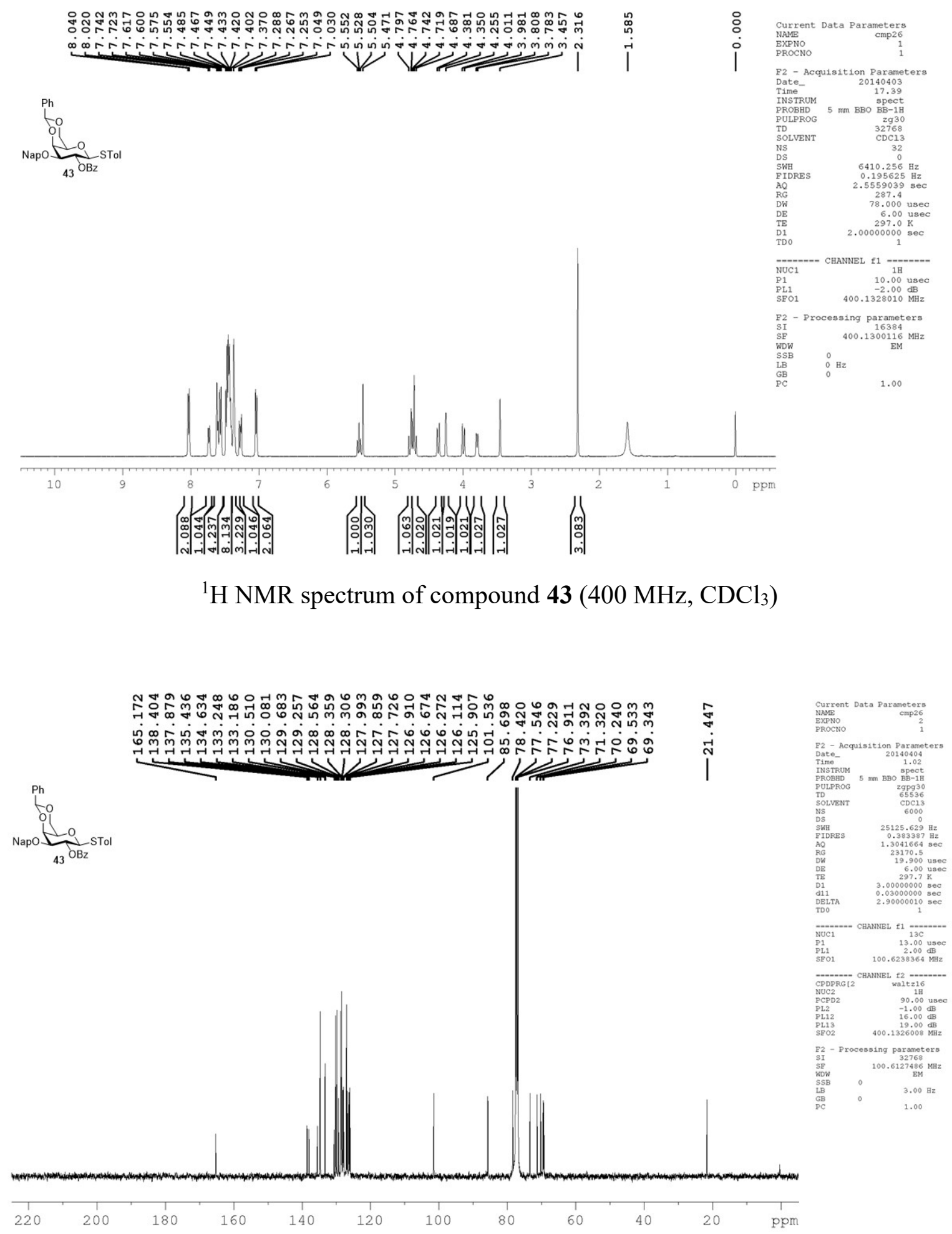

${ }^{13} \mathrm{C}$ NMR spectrum of compound $43\left(100 \mathrm{MHz}, \mathrm{CDCl}_{3}\right)$ 


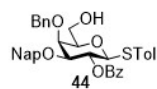
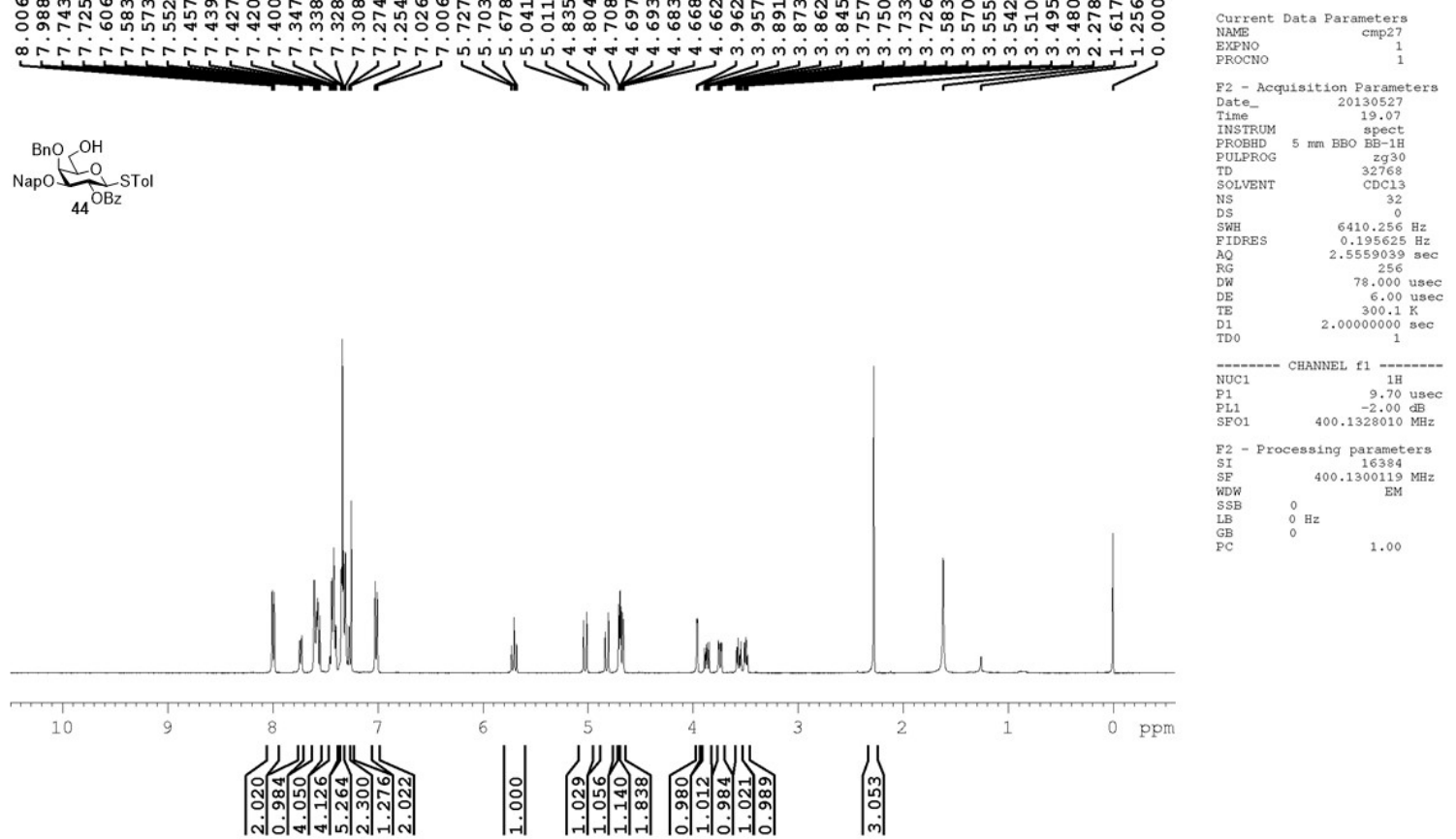

${ }^{1} \mathrm{H}$ NMR spectrum of compound $44\left(400 \mathrm{MHz}, \mathrm{CDCl}_{3}\right)$

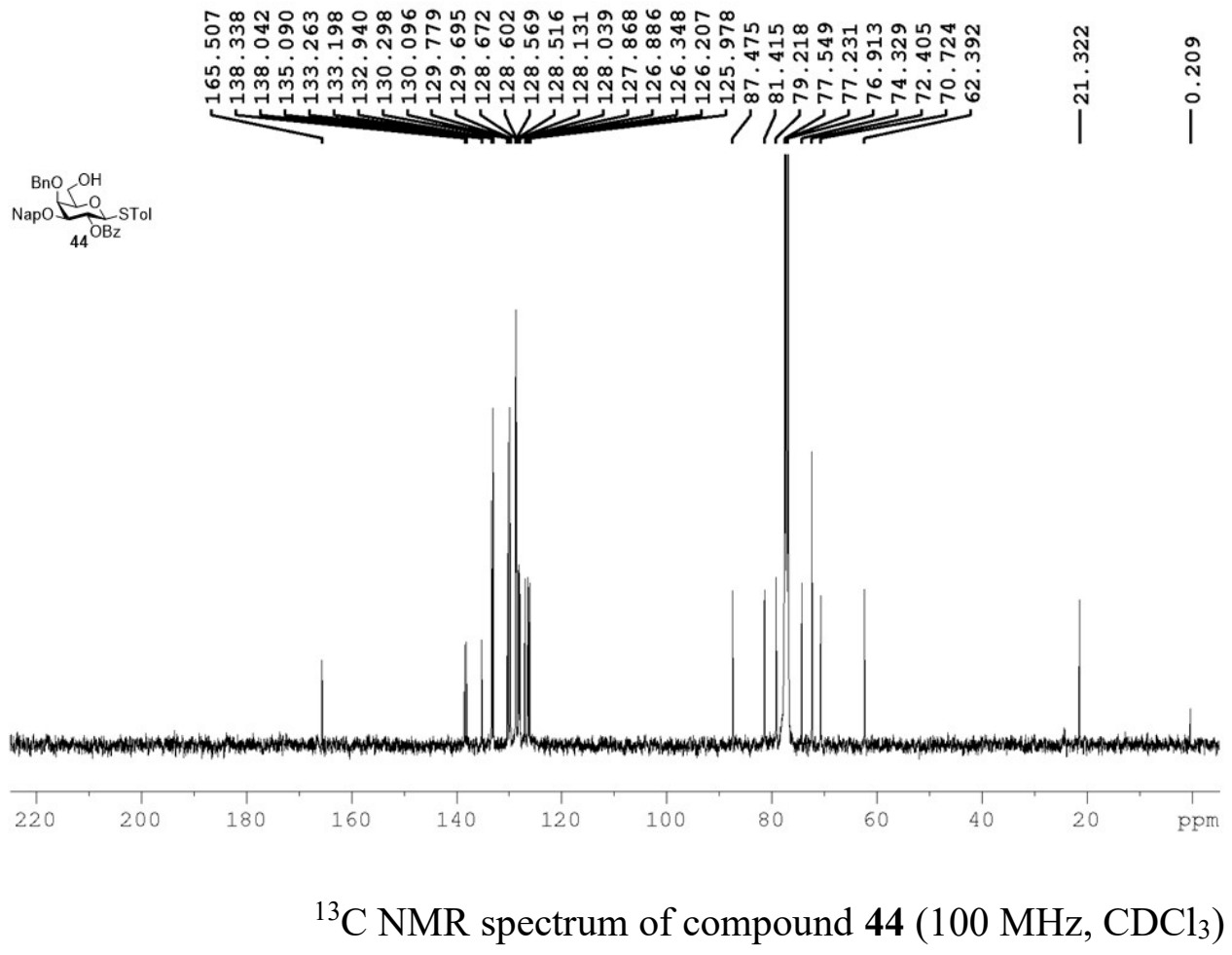




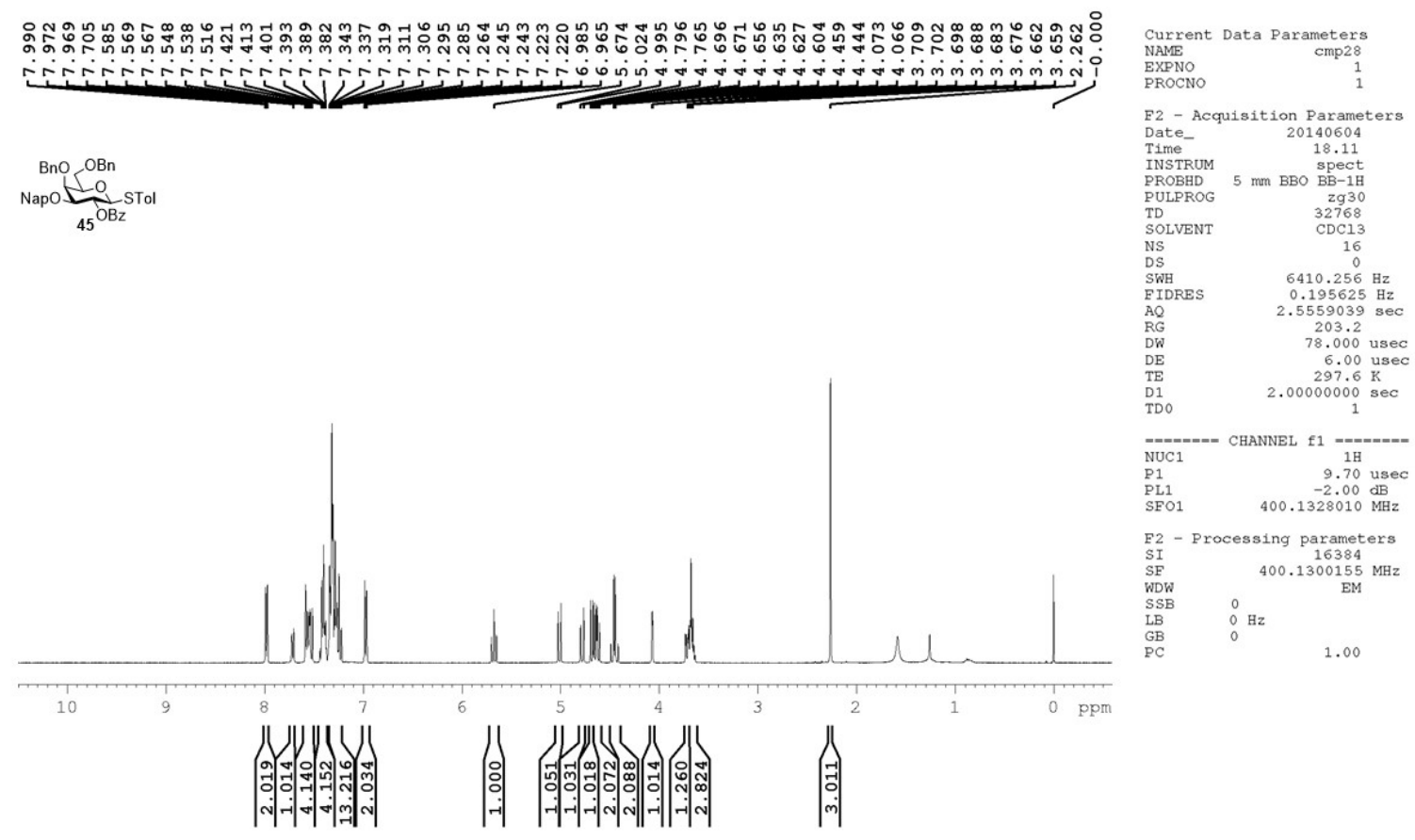

${ }^{1} \mathrm{H}$ NMR spectrum of compound $45\left(400 \mathrm{MHz}, \mathrm{CDCl}_{3}\right)$

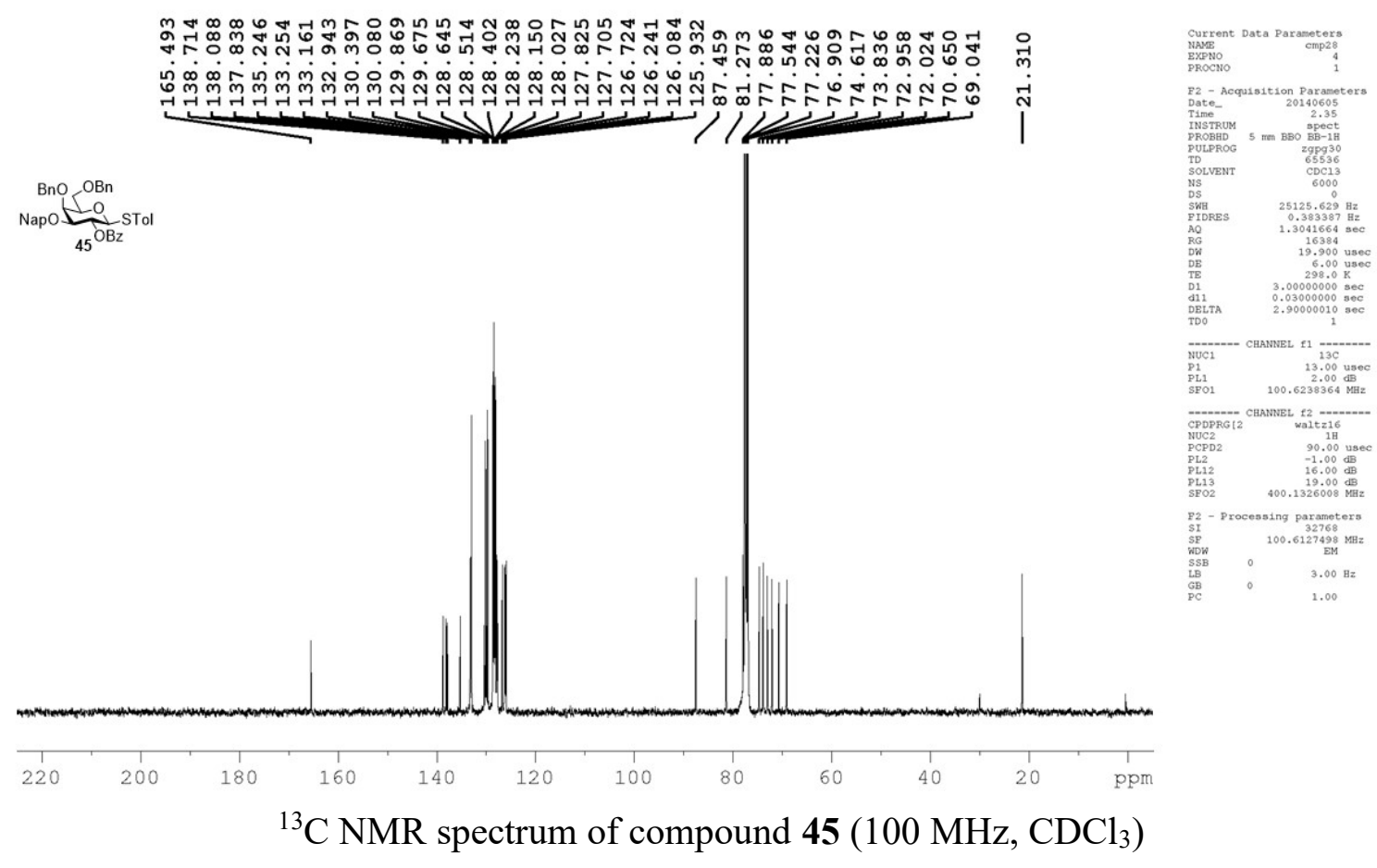




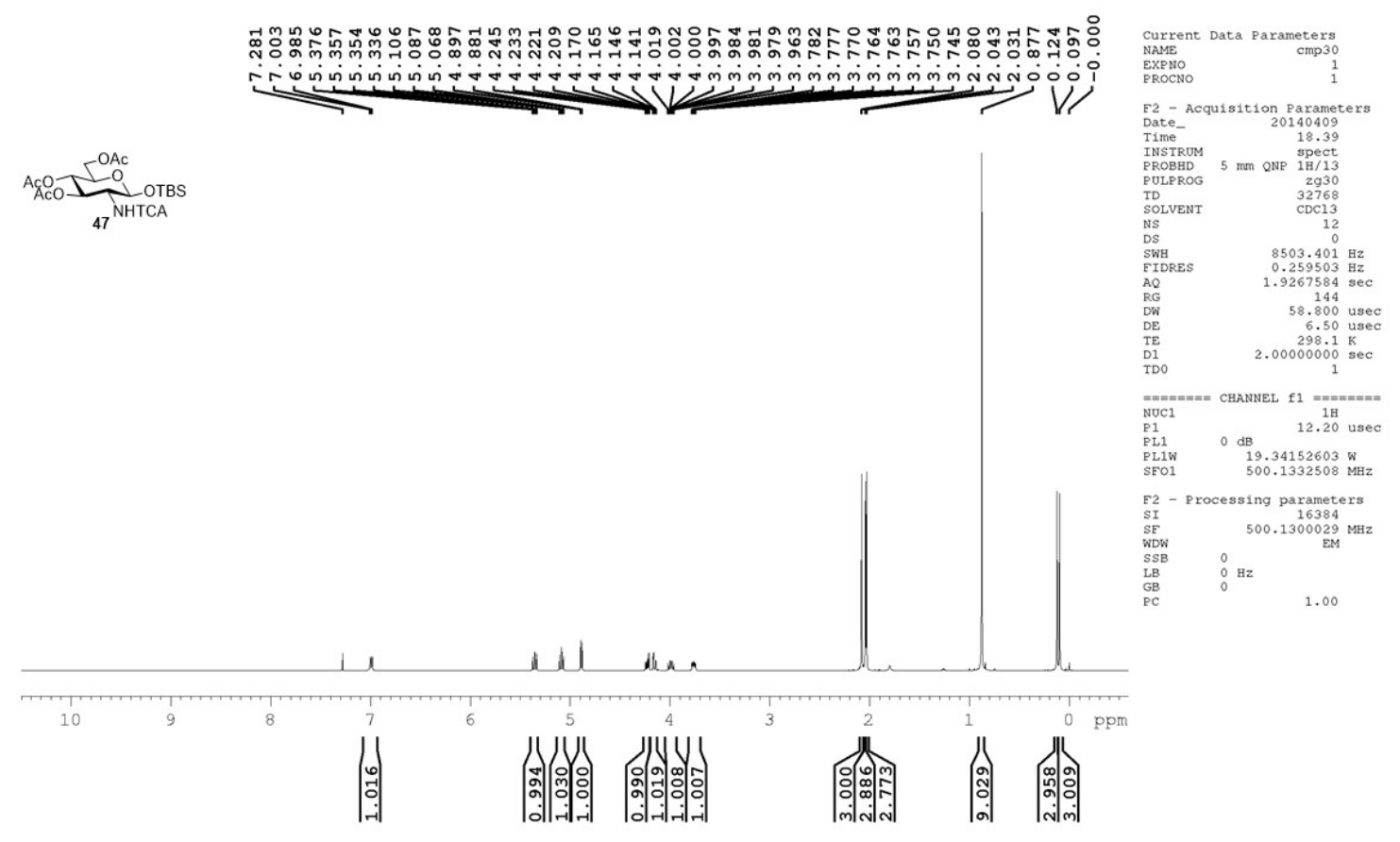

${ }^{1} \mathrm{H}$ NMR spectrum of compound $47\left(500 \mathrm{MHz}, \mathrm{CDCl}_{3}\right)$

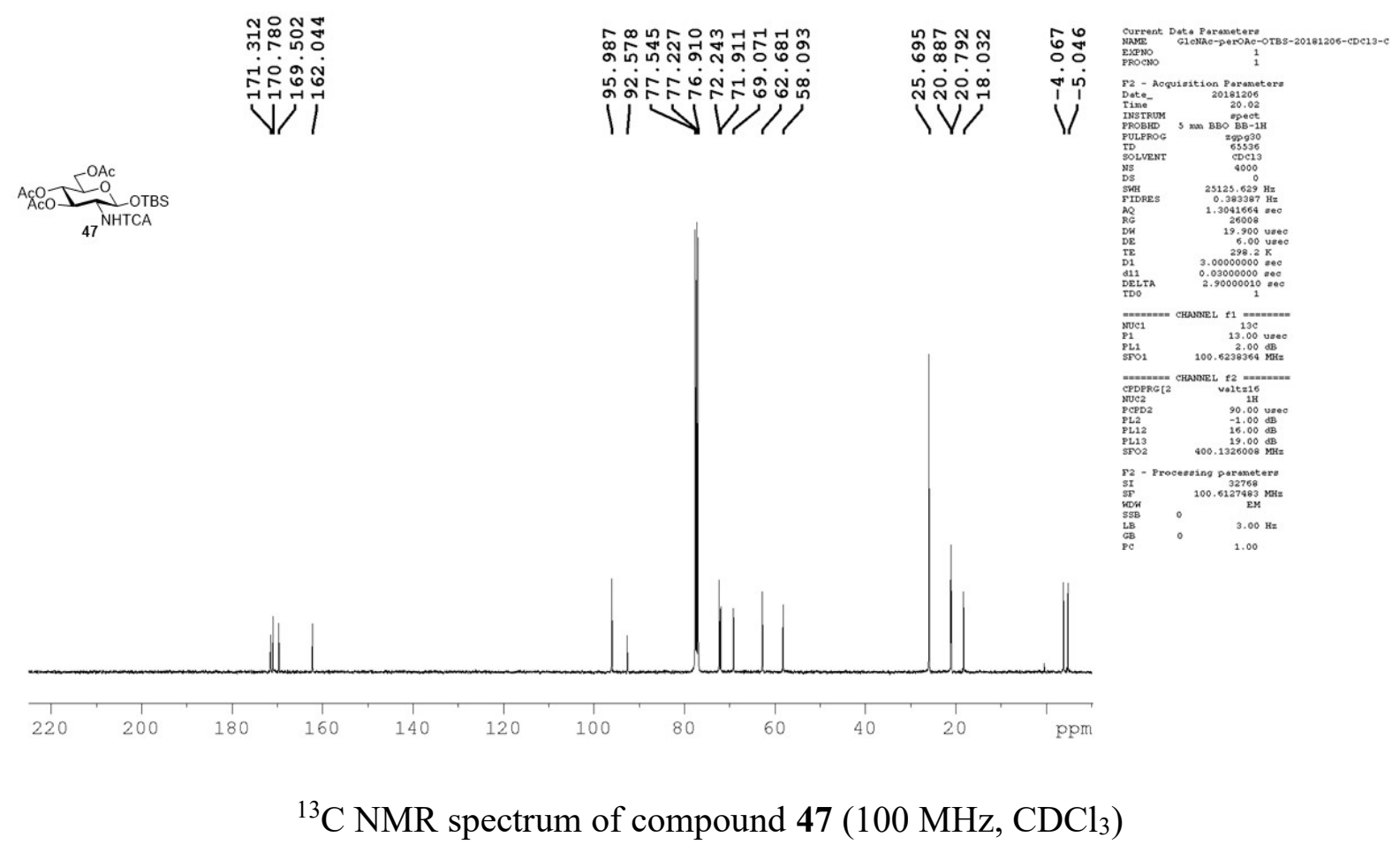



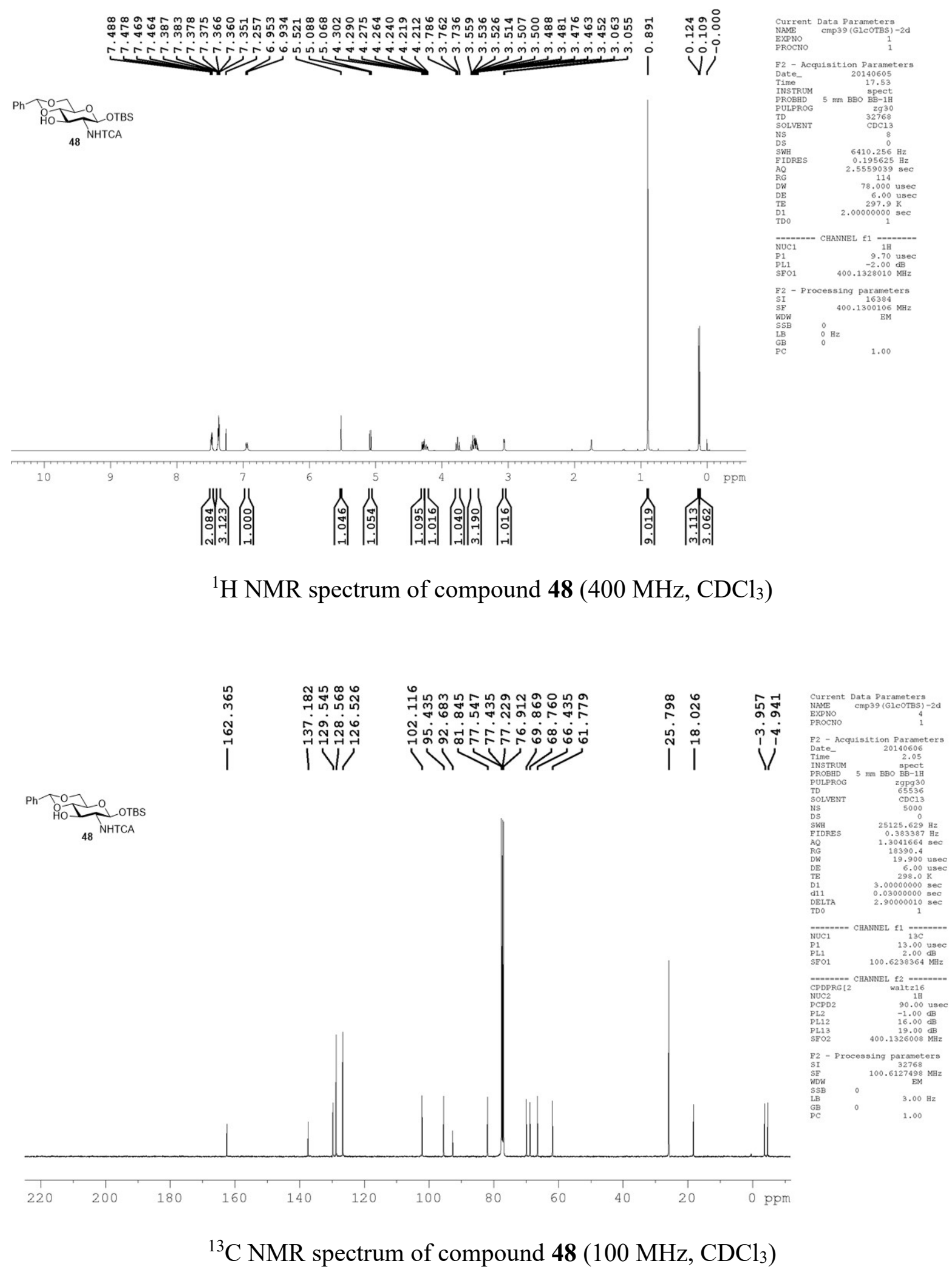


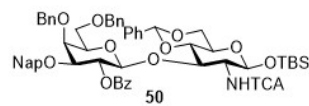
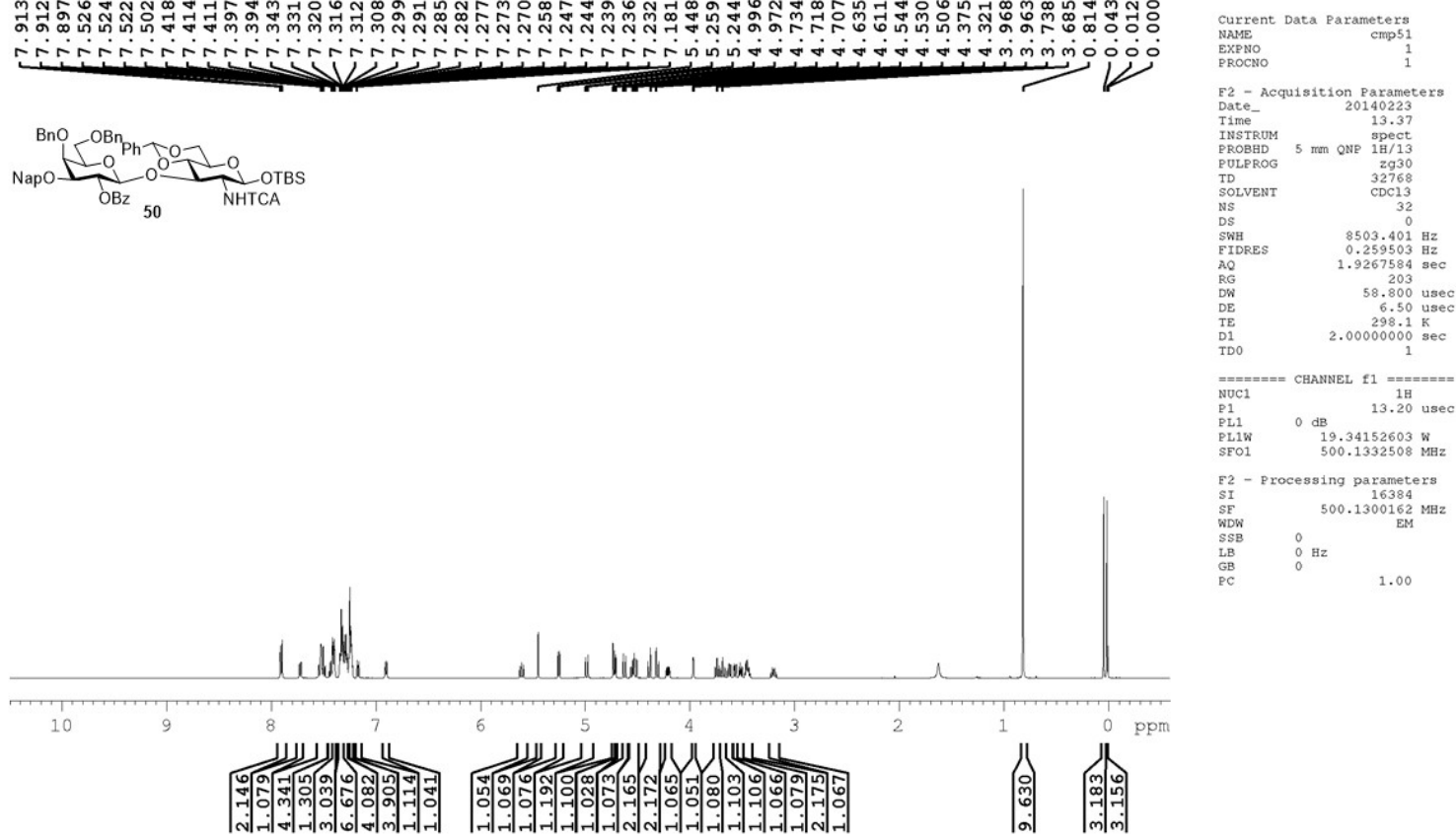

${ }^{1} \mathrm{H}$ NMR spectrum of compound $\mathbf{5 0}\left(500 \mathrm{MHz}, \mathrm{CDCl}_{3}\right)$

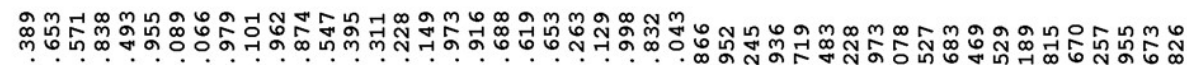
:

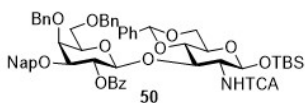

(1)

NapO ${ }_{\text {OBZ }} 50$ NHTCA
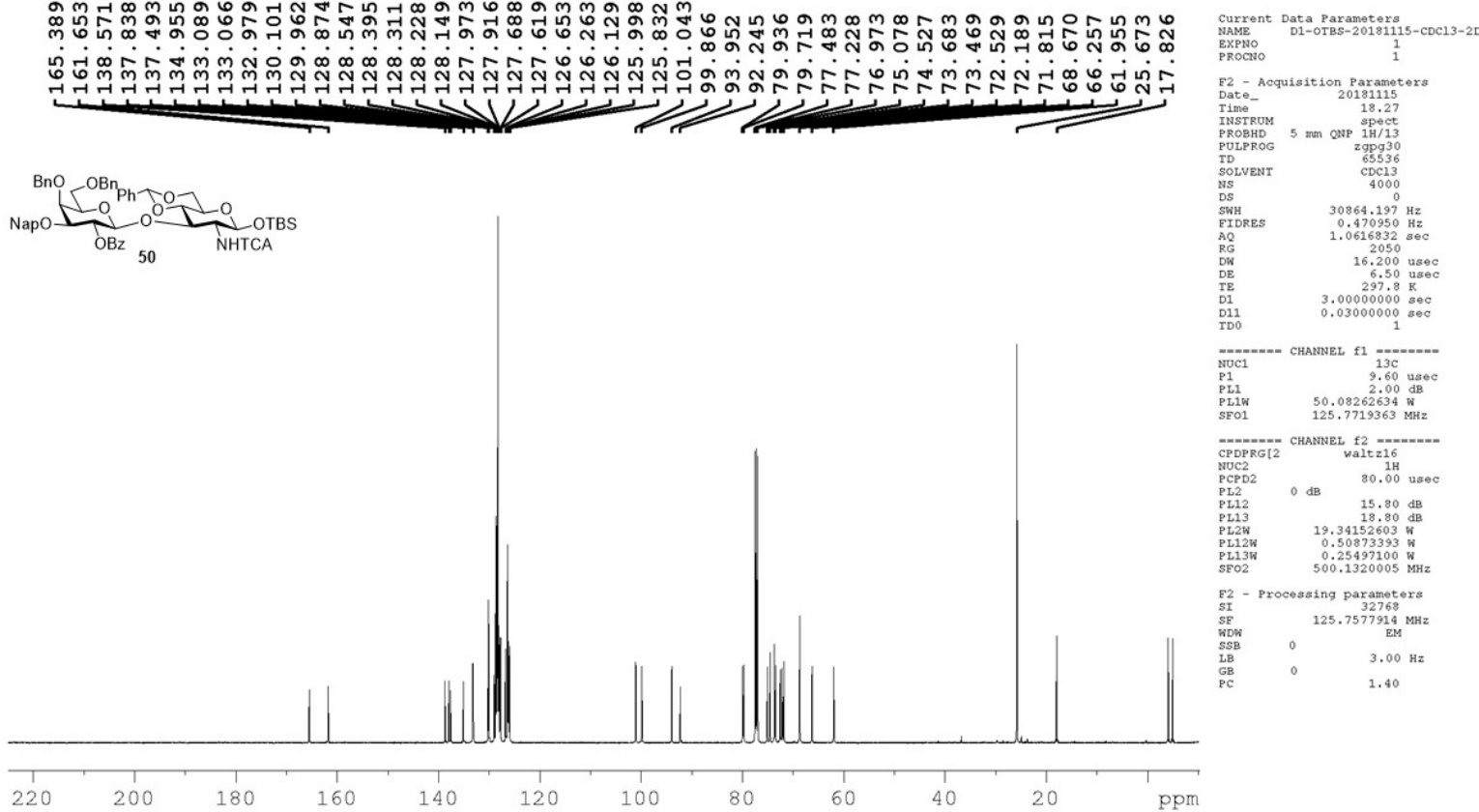

${ }^{13} \mathrm{C}$ NMR spectrum of compound $50\left(125 \mathrm{MHz}, \mathrm{CDCl}_{3}\right)$ 
${ }_{\mathrm{OBz}}{ }_{51}^{\mathrm{BnO}} \underbrace{\mathrm{OBn}}_{\mathrm{NHTCA}}$
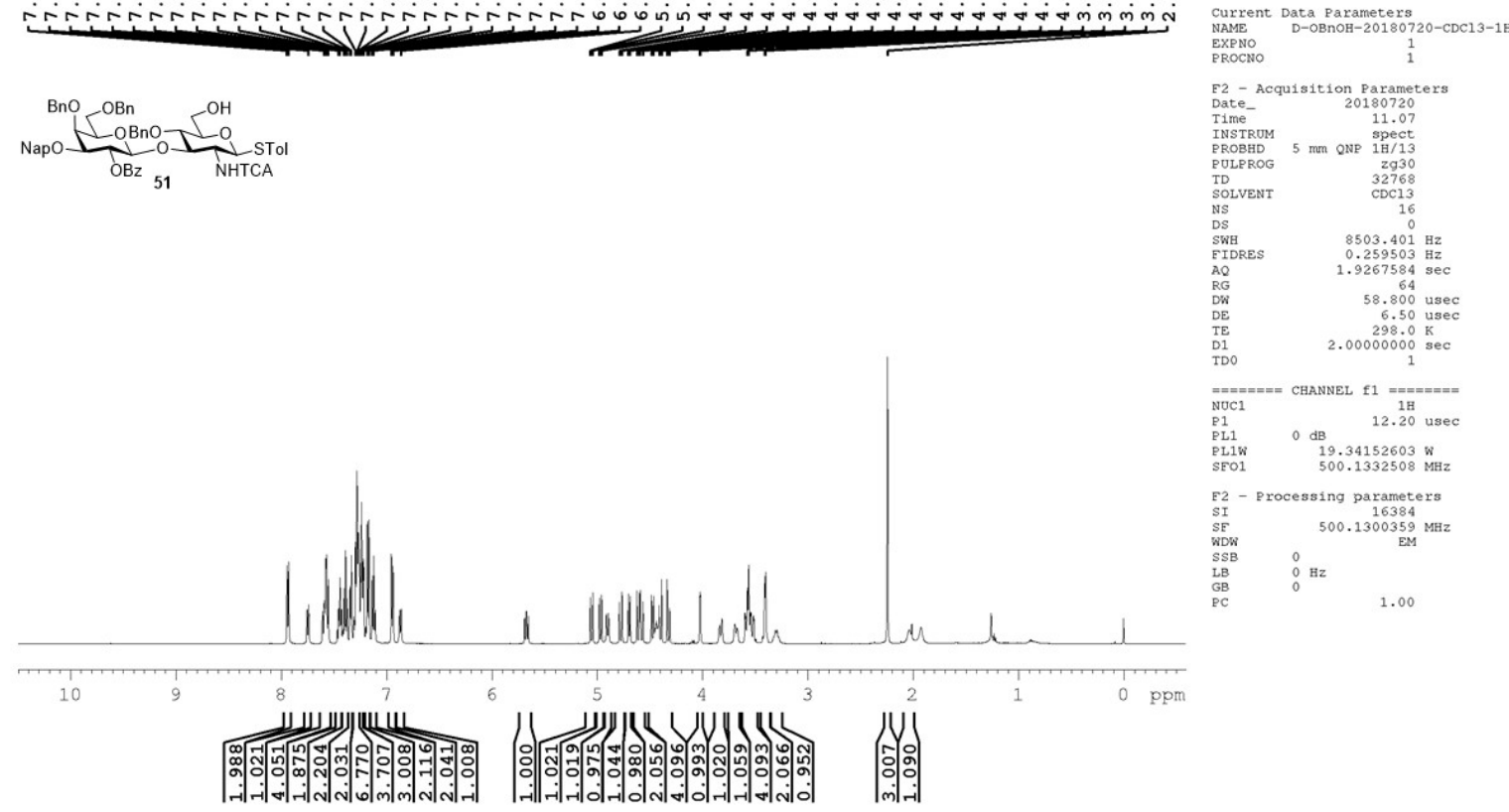

${ }^{1} \mathrm{H}$ NMR spectrum of compound $51\left(500 \mathrm{MHz}, \mathrm{CDCl}_{3}\right)$

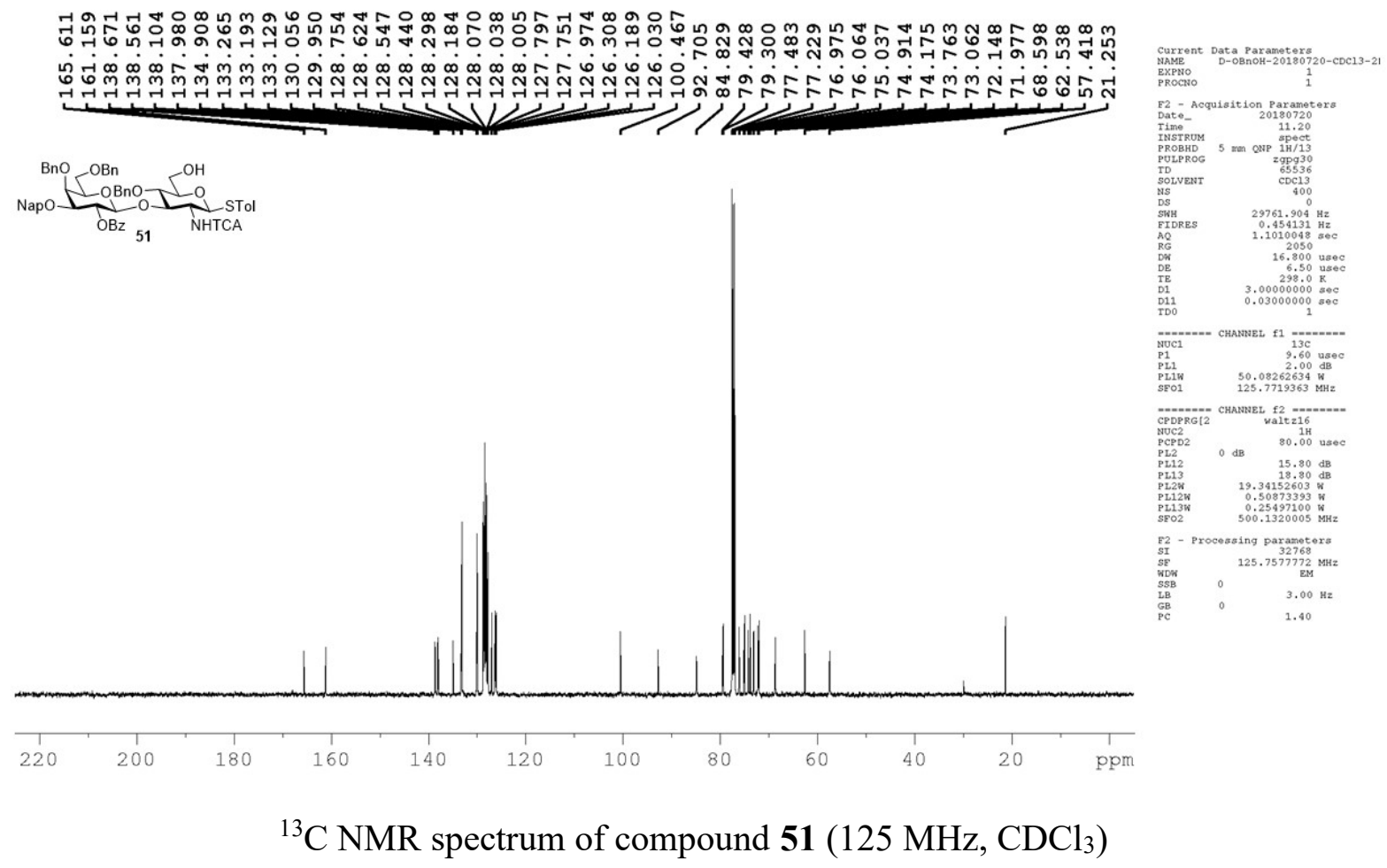



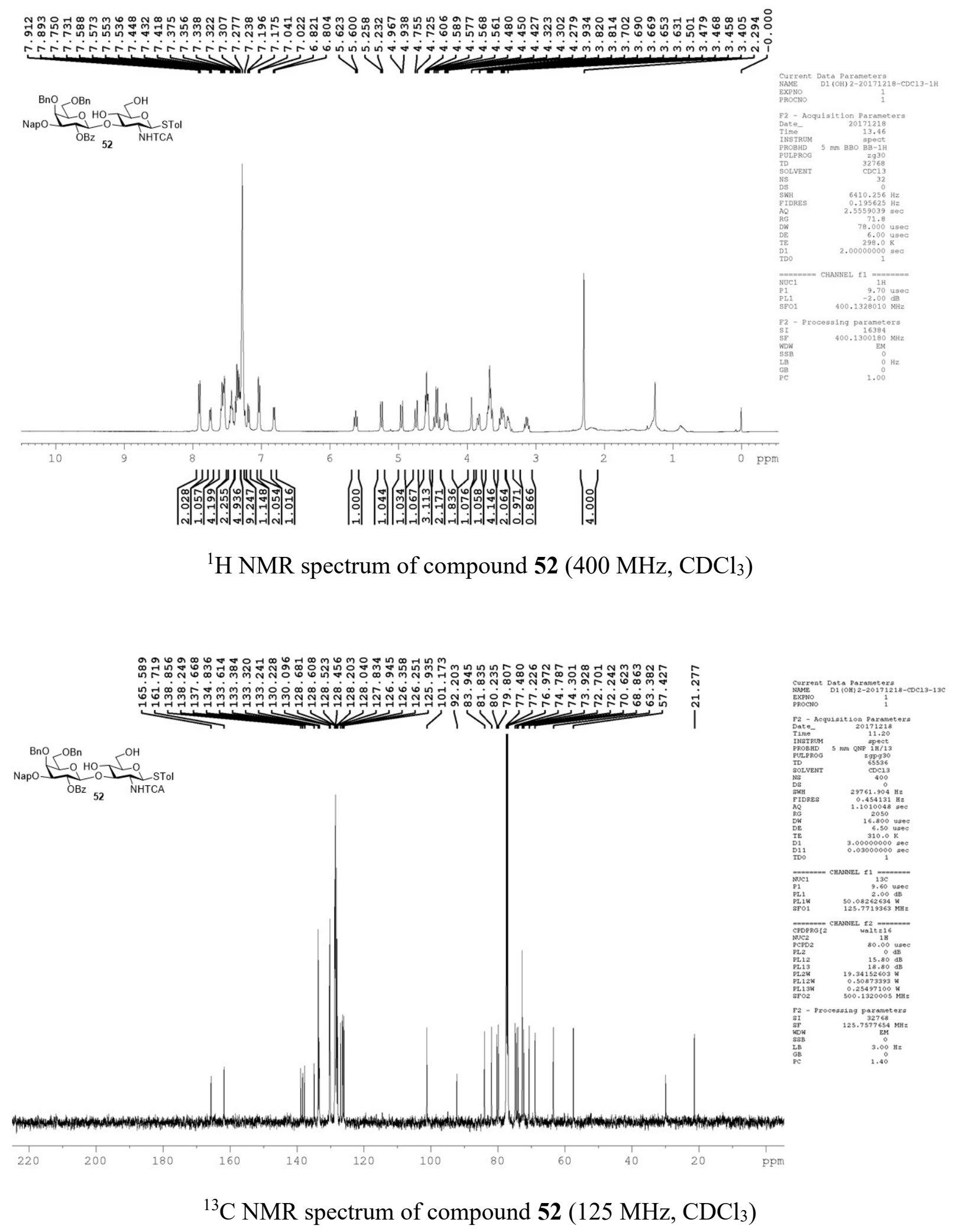


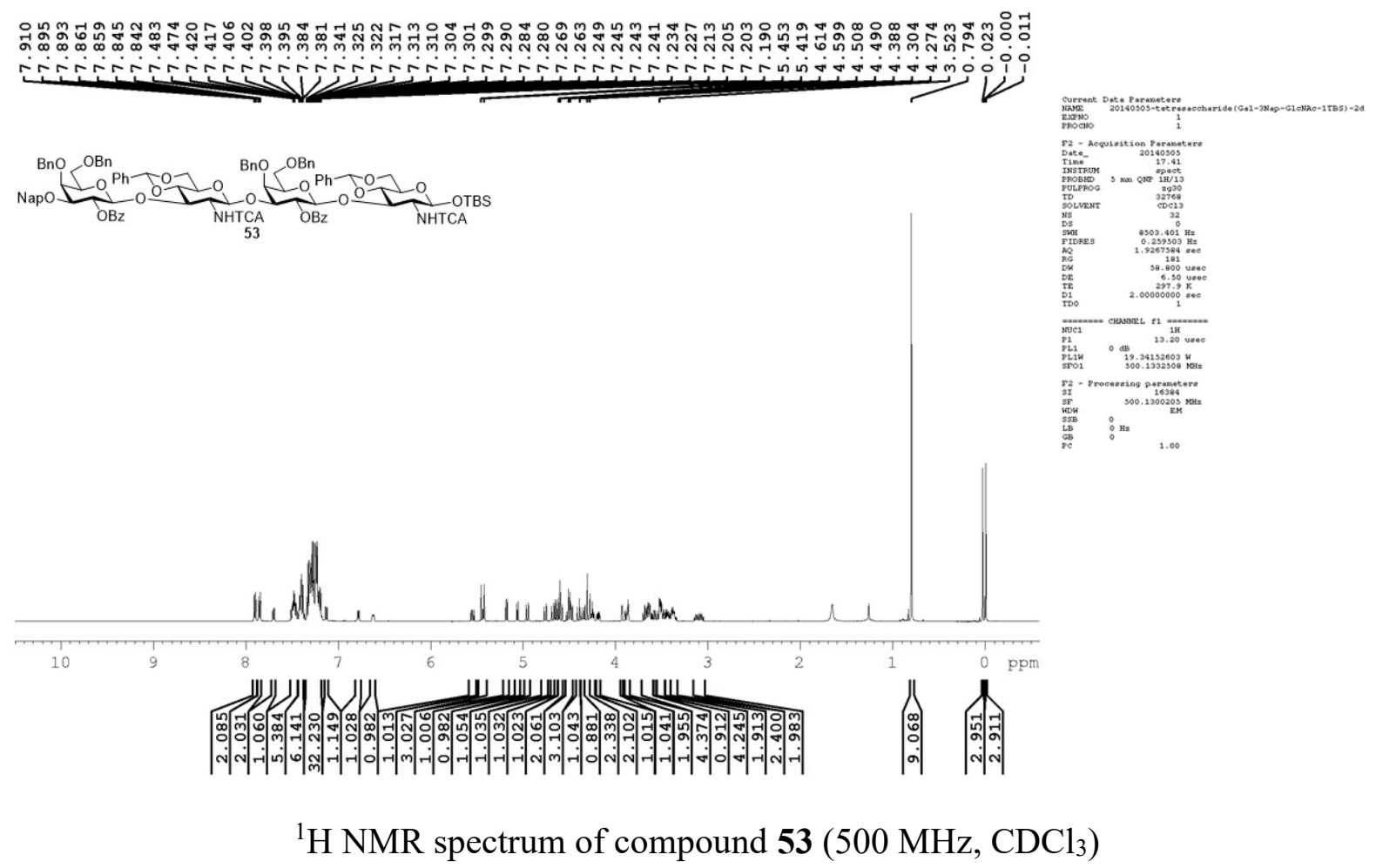

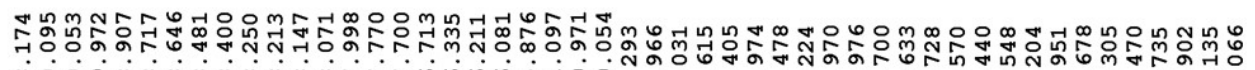

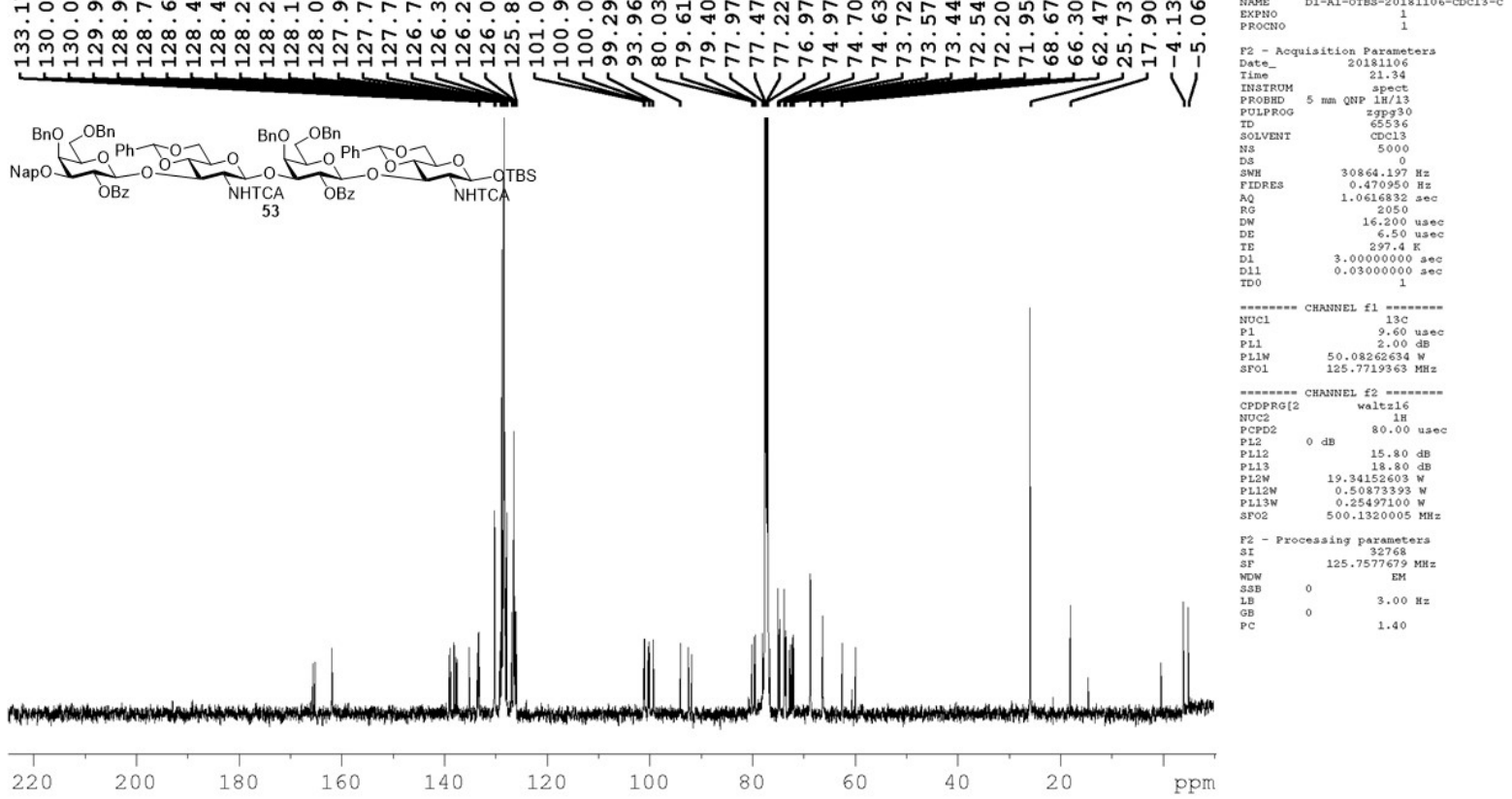

${ }^{13} \mathrm{C}$ NMR spectrum of compound $53\left(125 \mathrm{MHz}, \mathrm{CDCl}_{3}\right)$ 